\title{
ESNR 2016
}

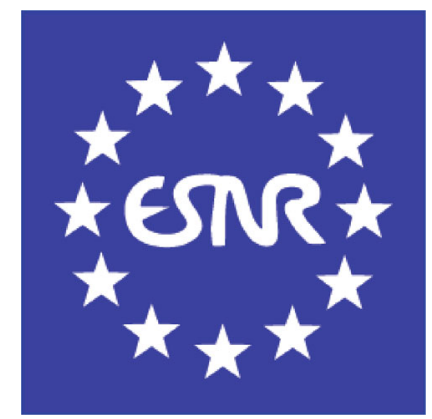

EUROPEAN SOCIETY OF NEURORADIOLOGY

Diagnostic and Interventional

$39^{\text {th }}$ ANNUAL MEETING

$23^{\text {rd }}$ Advanced Course in Diagnostic Neuroradiology $8^{\text {th }}$ Advanced Course in Interventional Neuroradiology

September 15 - 18

Belgrade, SERBIA

Conference Venue: CROWNE PLAZA HOTEL

PRESIDENT

Tatjana STOSIC-OPINCAL

\author{
Olav Jansen \\ Mario Muto \\ Bernhard Schuknecht \\ Marion Smits \\ Pia Maly Sundgren \\ Tarek Yousry \\ Dusan Damjanovic \\ Marija Jovanovic \\ Milos Lucic \\ Svetlana Milosevic-Medenica \\ Ivan Nikolic \\ Dragan Stojanov \\ Ivan Vukasinovic
}

SCIENTIFIC COMMITTEE

LOCAL ORGANIZING COMMITTEE 


\section{WELCOME ADDRESS}

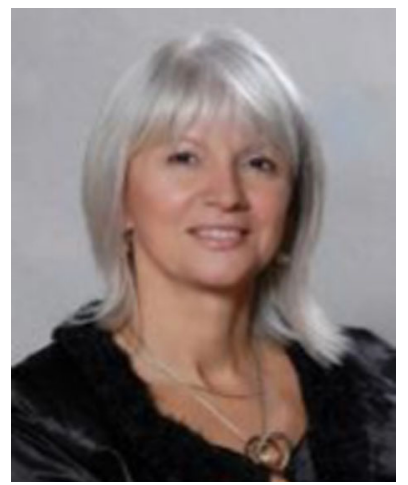

It is my great honor and pleasure to welcome you in Belgrade. This year Belgrade will host our 39th ESNR Annual Meeting, with 23rd Advanced Course on Diagnostic Neuroradiology and 8th Advanced Course on Interventional Neuroradiology. Courses will be held on September 15th, and Annual meeting from September 16th till September 18th.

This meeting will provide you with interesting scientific program enriched with multiple educational workshops, scientific lectures related to both, clinical problems and scientific topics. Numerous distinguished lecturers and experts from all around the world will join their efforts to present current topics and interesting cases, covering all fields of Neuroradiology. We invite you to take active participation during the sessions, since such meetings are a unique opportunity for the exchange of knowledge and experiences between colleagues from different countries and continents. The 39th ESNR Annual Meeting will offer the opportunity for younger radiologist to present their work and achievements through poster presentation and parallel scientific sessions.

After a hard working day, we offer you the opportunities to explore and enjoy Belgrade, the city which lays at the confluence of the Sava and the Danube rivers, through the social events that we have arranged for you. Belgrade is one of the oldest cities in Europe and has been an important crossroad since ancient times. It's the place where Western and Eastern Europe meet. Belgrade presents at the same time a mixture of a modern European metropole and an old historical town. Serbian people have made some greatest contributions to science, art and culture.

And finally, Belgrade is famous for its interesting night life and various types of restaurants with excellent music from jazz to folk.

We look forward to seeing you in Serbia, country of Nikola Tesla, Mihajlo Pupin, Ivo Andric, Emir Kusturica, Goran Bregovic, Novak Djokovic...

Prof. Tatjana Stosic-Opincal, MD

President of the $39^{\text {th }}$ Annual Meeting of the ESNR 


\section{The European Society of Neuroradiology - Diagnostic and Interventional Executive Committee}

President

Vice - President

Past President

Secretary General

Treasurer

Chairperson of the Subspecialty Committee Diagnostic Neuroradiology

Chairperson of the Subspecialty Committee Head and Neck Neuroradiology

Chairperson of the Subspecialty Committee Interventional Neuroradiology

Chairperson of the Subspecialty Committee Paedriatic Neuroradiology

Chairperson of the Subspecialty Committee Spine Neuroradiology, diagnostic and interventional

Secretary of the Institutional Council
MajdaM.Thurnher

Alex Rovira Canellas Turgut Tali

Andrea Rossi

Johan Van Goethem

Tarek Yousry

Bernhard Schuknecht

Olav Jansen

Pia Sundgren

Mario Muto

Michael Forsting

\section{Institutional Council}

Australia
Belgium
Bosnia Herzegovina
Bulgaria
Croatia
Denmark
Finland
France
Germany
Greece
Hungary
Ireland
Italy

M. Changer

E. Achten

Z. Merhemic

L. Penev

S. Jankovic

V. A. Larsen

R. Vanninen

A. Bonafé

C. Groden

A. Gouliamos

P. Barsi

S. Looby

A. Rossi
Latvia

Luxembourg

The Netherlands

Norway

Poland

Portugal

Serbia

Slovenia

South Africa

Sweden

Switzerland

Turkey

United Kingdom
G. Krumina

G. Dooms

M. Smits

P. Due Tonnessen

J. Walecki

P. L.F. Vilela

T. Stosic - Opincal

K. Surlan Popovic

S. Candy

R. Siemund

K. O. Lovblad

I. Saatçi

K. Lobotesis

\section{Institutional Members}

British Society of Neuroradiologists Bulgarian Society of Neuroradiology Croatian Society of Neuroradiology Danish Society of Neuroradiology Dutch Society of Neuroradiology Finnish Society of Neuroradiology French Society of Neuroradiology German Society of Neuroradiology Hungarian Society of Neuroradiology Italian Society of Neuroradiology Serbian Society of Neuroradiology South African Society of Neuroradiology
Latvian Society of Neuroradiology Norwegian Society of Neuroradiology Portuguese Society of Neuroradiology Spanish Society of Neuroradiology Swiss Society of Neuroradiology Turkish Society of Neuroradiology Association of Radiology of Bosnia Herzegovina, division of Neuroradiology Neuroradiology section of the Hellenic Radiological Society Australian and New Zealand Society of Radiology Irish Society of Neuroradiology Slovenian Society of Neuroradiology Swedish Society of Neuroradiology 


\section{Committees of the ESNR}

Diagnostic Neuroradiology

Tarek Yousry (Chair)

Marion Smits

Nuria Bargallo Alabart

Head and Neck Neuroradiology

Bernhard Schuknecht (Chair)

Sotirios Bisdas

Katarina Surlan Popovic

Interventional Neuroradiology

Olav Jansen (Chair)

Francesco Causin

Jan Gralla

Alain Bonafé

Maria Luisa Biscoito

Jen Fiehler

Pediatric Neuroradiology

Pia Maly Sundgren (Chair)

Maria Argyropoulou

Bård Nedregaard

Spine Neuroradiology

Mario Muto (Chair)

Alessandro Cianfoni

Marc-André Weber

\section{Rules}

Philippe Demarel

Andrea Rossi

Seamus Lobby

Marek Sasiadiek

Alexandre Krainik

\section{Scientific Programme}

Paul M. Parizel (Chair)

Marek Sasiadek

Friedhelm Zanella

Mario Muto

Majda Thurnher

Tatjana Stosic - Opincal

\section{Membership}

Cem Calli (Chair)

Paulina Due - Tonnessen

Zulejha Merhemic

\section{Social Media}

Nuria Bargallo Alabart (Chair)

Efrosini Papadaki

Yelda Ozsunar Dayanir

Guido Wilms

Luc van den Hauwe

\section{ESONR}

Turgut Tali (Chair)

Majda M. Thurnher

Andrea Rossi

Olav Jansen

Pia Maly Sundgren

Bernhard Schuknecht

Tarek A. Yousry

Mario Muto

Johan Van Goethem

\section{Audit}

Marek Sasiadek (Chair)

Zulejha Merhemic

Gian Antonio Pellicano

\section{Founding Members}

Jacques Bories

Giovanni Ruggiero

Vittorio Bernasconi

Herbert Backmund

Ryszard Chrzanowski

Luis Escudero

Torgny Greitz

Louis Jeanmart

Jan Jirout

Francesco Smaltino

Jean Metzger

André Thibaut

Gianni Boris Bradac

Roland Oberson

Desmond Hawkins

Lourens Penning

Bengt Liliequist

Sten Cronquist

Peter Huber

Milan Roth

George Du Boulay

Juan Solé-Llenas

Richard Hoare

Angelo Passerini

Hans Hacker

Kjell Bergstrom

Luc Picard

Georges Salamon

Alberto Calabrò

European Exchanging Program Martin Schumacher (Chair)

Tarek Yousry

Rüdiger von Kummer

Jordi Ruscalleda Nadal
Members-in-training

Julia Frühwald - Pallamar (Chair)

Reuben Grech

Ana Filipa Geraldo

Christian Weisstanner

Scientific Award

Rüdiger von Kummer (Chair)

Per Hjalmar Nakstad

Isil Saatci

Marco Leonardi

Sven Haller

Joanna Wardlaw

Nadine Girard 


\section{THE ESNR AWARDS: THE EUROPEAN NEURORADIOLOGY AWARDS IN DIAGNOSTIC AND INTERVENTIONAL NEURORADIOLOGY AND THE LUCIEN APPEL PRIZE OF THE ESNR}

In 2008, the Executive Committee of the ESNR decided to introduce two new scientific prizes for young neuroradiologists, alongside the existing Lucien Appel Prize of the ESNR, which is awarded annually for the best research paper. The new prizes were created in honour of the Pioneers and Past Presidents of European Neuroradiology, and are awarded for the best papers in diagnostic and interventional neuroradiology.

In 2012, the Executive Committee of ESNR decided to give the name of one of the Pioneers of the society and the name of one of its Past Presidents in an order as "ESNR Awards in Honor of", and to present their short CV and pictures at the Awards Ceremony during the ESNR Annual Congress or during the Symposium Neuroradiologicum.

Among those awards, the proposal of Springer to institute the "ESNRSpringer Award" was also accepted. $€ \mathbf{1 0 0 0}$ will be awarded by Springer to the first (ESNR's Full, Associate, Junior or Institutional Member) author of the most cited manuscript published in the previous two years from January to December in the journal "Neuroradiology". Citations of the manuscripts will be obtained from SCI on first workday of September, at 17.30 of each year.

\section{Rules of participation}

Article 1: The three prizes reward the achievements of young scientists, under the age of 40 years on the day of application, working in the field of neuroradiology in a European centre and having been accepted as Full, Associate, Junior, or Institutional Member of the ESNR.

Article 2: The prizes, each to the amount of $€ 4000$, are awarded every year at the Awards Ceremony during the annual congress of the ESNR, or during the Symposium Neuroradiologicum when the ESNR Annual Congress does not take place. The Awards Ceremony is a respected event during each congress; other simultaneous ESNR sessions are not allowed, and the ceremony may not be split in two or more parts. The Award Ceremony is conducted by the Chair of the Awards Committee, who also introduces the awardees and presents the prizes together with the ESNR President.

Article 3: The candidate must be the first author of an original work in the fields of research in neuroradiology: diagnostic or interventional neuroradiology. Applications can be placed for a scientific work that was: a. published online or in print in a peer-reviewed scientific journal listed in SCI within the last 12 months before the date of submission, or b. submitted, still unpublished, accepted or not yet accepted.

The candidate must not have received any other prize for the same work, and must not have received the ESNR award of the same category previously.

Article 4: All submissions must be in English and should include:

- application letter indicating the title of the scientific paper upon which the application is based, and reporting its status if not yet published;

- the scientific paper (unpublished or to be submitted);

- the full Curriculum Vitae and a photo of the candidate.

These documents must be exclusively submitted, as electronic attachments, via e-mail to the address indicated below. Please note that each submitted article and its relevant images should be included in a separate attachment.
By submitting the work the author agrees, if declared the winner, to present the work at the next ESNR Annual Congress or Symposium Neuroradiologicum. Should the winner be unable to participate because of proven urgent reasons, the presentation can be made by a co-author of the same paper. If no presentation is made, the prize money will not be awarded. Payment of the prize money is made after the relevant ESNR Annual Congress or Symposium Neuroradiologicum has taken place.

Article 5: A single author can apply for more than one prize with scientific papers on clearly different topics as confirmed by the Awards Committee.

Article 6: Authors are requested to indicate to which of the three awards categories each scientific paper is addressed. If the Awards Committee considers another category to be more appropriate, the submission may be reclassified.

Article 7: A Scientific Jury will assess and rate the submitted papers. The Jury is composed of the members of the Awards Committee, plus four internationally known specialists in neuroradiology, who will rate each application independently from each other. If the rating does not give clear results, the prize-winners will be identified by consensus of all jury members.

The members of the Scientific Jury should declare not to have a conflict of interests. In case of conflict of interests, the juror is excluded from the Scientific Jury for the current year and specific prize category, and is replaced by another juror of equal scientific standing.

The Chair of the Awards Committee shall be invited as a Faculty member to the ESNR Annual Meeting or Symposium Neuroradiologicum where the award ceremony takes place.

Article 9: The ESNR will advertise the prizes by a dedicated newsletter and in the society website, as of January $1^{\text {st }}$ each year. The deadline for applications is set at March $1^{\text {st }}$ each year. The Award Committee will designate the laureates after receiving the evaluations of the jurors, as a rule by May ${ }^{\text {st }}$. Should the Award Committee decide that, in any category, no submission meets sufficient quality requirements, no prize will be awarded in that category.

Article 10: All candidates will be notified in writing of the result of their application. The laureates will be invited to give a six-minute oral presentation at the Award Ceremony during the ESNR Annual Congress, or Symposium Neuroradiologicum when the ESNR Annual Congress does not take place. The congress registration fee of the laureates is waived; the travel expenses will be reimbursed.

The names of the laureates, as well as the titles and abstracts of their scientific works, will be placed on the ESNR website and published in the society pages of Neuroradiology. In case the winning paper is still unpublished, the authors agree that "Neuroradiology", the official ESNR journal, shall have the right of publication after peerreview.

Article 11: All possible questions and issues regarding the awarding of the Prizes shall be dealt with at the discretion of the Awards Committee and the ESNR Executive Committee. The decision of the ESNR Executive Committee is final. 
Article 12: Candidacy implies full acceptance of the rules.

Please send your submissions to the attention of the ESNR Secretary General

E-mail: info@esnr.org

The object of the email should read: ESNR award submission.

\section{THE DEADLINE FOR RECEIPT OF SUBMISSIONS IS MARCH $1^{\text {ST }}$}

ESNR Annual Congress, Advanced Course in Diagnostic Neuroradiology and Advanced Course in Interventional Neuroradiology of the ESNR.

The European Society of Neuroradiology holds each year a scientific congress at a place and date designated by the Executive Committee. On the day before the congress, Advanced Courses in Diagnostic Neuroradiology and Interventional Neuroradiology are organized in parallel sessions. The business meeting (General Assembly) of the Society is held in conjunction with this annual scientific congress.

Since its creation in 1969, the ESNR has organized 39 annual congresses. This year the $23^{\text {rd }}$ Advanced Course in Diagnostic Neuroradiology and the 8th Advanced Course in Interventional Neuroradiology will be held on the day before the congress. In recognition of the importance of the Symposium Neuroradiologicum, which is held every four years, no scientific congress of the ESNR is held when the Symposium takes place in Europe.

\section{Future Annual Meetings}

\section{0th Congress}

September 2017

Malmö, Sweden

President: Pia Maly Sundgren

\section{1st Congress}

September 2018

Rotterdam, the Netherlands

President: Marion Smits

\section{Future Symposia}

\section{Symposium Neuroradiologicum}

March 19-24, 2018

Taipei, Taiwan

President: Wan-Yuo Guo

\section{The European Course in Neuroradiology}

The European Course in Neuroradiology has been a story of success ever since the first course in Toulouse in 1984. More than 1000 young neuroradiologists have completed the cycle of three courses over the past 21 years. The ECNR was conceived as a means to create a common and shared culture, common meeting points and a common standard of knowledge. The form that was adopted and used for the coming 6 cycles, each consisting of three courses, included the major bodies of knowledge as described by the headlines; intracranial nervous system, the spine and spinal cord and the base of the skull, maxillofacial and head and neck neuroradiology.

\section{European School of Neuroradiology (ESONR) - the new concept}

The ESONR - European School of Neuroradiology - is the most important and complete training programme organized by the ESNR - European Society of Neuroradiology within the main frame, and in partnership with, the ESOR - European School of Radiology. The purpose of this training and education program is based on the vision of what the ESNR considers the range of competences and skills that are the basis of a high qualified neuroradiological activity. The program is designed to offer a pathway to reach such professional and cultural levels. The final points will be the examinations to be certificated at the different levels and in the different branches of this discipline.

Courses are organized at 3 different levels: 1st Level: ESOR - Galen Foundation Courses in Neuroradiology, 2nd level: ECNR Course in Neuroradiology, Diagnostic and Interventional and finally 3rd level: ESNR Advanced Courses of Higher Qualification in Interventional Endovascular Neuroradiology, Interventional Spine Neuroradiology, Advanced Diagnostic Neuroradiology and Paediatric Neuroradiology.

ECNR - European Course in Diagnostic andinterventional Neuroradiology

The ECNR is the fundamental Neuroradiology course aimed at neuroradiologists, established or in training. It is based on cycles of four courses (modules), each lasting five days, dedicated to diagnostic and interventional neuroradiology. The full cycle is considered complete after the attendee has participated in all four modules, which can be done in a single cycle or in different cycles.

The scientific content of the course is determined by the Education Committee of the European Society of Neuroradiology, taking into account international standards and guidelines for training in diagnostic and interventional neuroradiology. The participating educators will be internationally renowned European experts, to be selected on the basis of their scientific background and educational skills to ensure high- quality lectures and interactive case discussions.

The following topics have been chosen, each to be covered in five full days of lectures and workshops:

- Anatomy, congenital malformations and genetics.

- Trauma, Infection, Inflammation and Degenerative Disease

- Tumours of the Brain and Spinal Canal

- Vascular Disease of the Brain and Spinal Canal 
Seventh Cycle - Scientific Director: Guido Wilms (Leuven)

First Course: Embryology, Anatomy, Malformations - Crieff, October 4-11, 2002 -Local Director:Wendy J. Taylor

Second Course: Tumours of the CNS - Riga, April 11 - 18, 2003 - Local

Director: Cosma Andreula

Third Course: Vascular Disease of the CNS - Malta, October, 24 30,2003 - Local Director: Athanassios Gouliamos

Fourth Course: Trauma and Degenerative Disease of the CNS Riga, April 23-27, 2004 -Local Director: Johan Van Goethem

Eighth Cycle -Scientific Director: Ernst-Wilhelm Radü (Basel)

First Course: Embryology/Anatomy/Malformations/Genetics - Basel, October 22-26, 2004

Second Course: Tumors of the CNS - Basel, March 18-22, 2005

Third Course: Vascular Diseases of the CNS - Basel, October 21-25, 2005

Fourth Course: Trauma/Degenerative Diseases of the CNS - Basel, March 10-14, 2006

Ninth Cycle - Scientific Director: Ernst-Wilhelm Radü (Basel)

First Course: Embryology/Anatomy/Malformations/Genetics - Basel, October 20 - 24, 2006

Second Course: Tumours of the CNS - Basel, March 23 - 27, 2007

Third Course: Vascular Diseases of the CNS - Basel, November 2 - 6, 2007

Fourth Course: Trauma/Degenerative Diseases of the CNS - Basel, April $11-15,2008$

Tenth Cycle - Scientific Directors: Massimo Gallucci (L'Aquila), AlexRovira (Barcelona)

First Course: Embryology/Anatomy/Malformations/Genetics Tarragona, October $10-14,2008$

Second Course: Tumors and Tumor-like Vascular Lesions- Rome, March 20 - 24, 2009

Third Course: Vascular Diseases - Tarragona, October 9-13, 2009

Fourth Course: Trauma/Degenerative/Metabolic/Inflammatory Diseases Rome, March 19-23, 2010

Eleventh Cycle - Scientific Directors: Massimo Gallucci (L'Aquila), Alex Rovira (Barcelona)

First Course: Embryology/Anatomy/Malformations/Genetics Tarragona, November 5-9, 2010

Second Course: Tumors and Tumor-like Vascular Lesions- Rome, March 25-29, 2011

Third Course: Vascular Diseases - Tarragona, October 28 - November 1, 2011 Fourth Course: Trauma/Degenerative/Metabolic/Inflammatory Diseases Milano, April 12-16, 2012

Twelfth Cycle - Scientific Directors: Athanasios Gouliamos (Athens), E. Turgut Tali (Ankara)

First Course: Embryology/Anatomy/Development and Malformations of the CNS - Antalya, November 1-6, 2012

Second Course: CNS Tumors - Athens, April 8 - 13, 2013

Third Course: Vascular Diseases - Antalya, November 3-7, 2013

Fourth Course: Trauma/Degenerative/Metabolic/Inflammatory Diseases -

Athens, April6-10, 2014
Thirteenth Cycle - Scientific Directors: Athanasios Gouliamos (Athens), E. Turgut Tali (Ankara)

First Course: Embryology/Anatomy/Development and Malformations of the CNS - Antalya, November 9-13, 2014

Second Course: CNS Tumors - Rhodes, April 14-18, 2015

Third Course: Vascular Diseases - Izmir, November, 2015

Fourth Course: Trauma/Degenerative/Metabolic/Inflammatory Diseases Greece, April, 2016

Fourteenth Cycle - Scientific Directors: Majda M. Thurnher (Vienna), Johan Van Goethem (Antwerp)

First Course: Embryology/Anatomy/Development and Malformations of the CNS - Dubrovnik, October 2016

Second Course: CNS Tumors - Antwerp, April 2017

\section{Neuroradiology}

Neuroradiology, published by Springer Verlag, was founded as the official organ of the European Society of Neuroradiology in 1970 . Since 1981, Neuroradiology is also the official organ of the Japanese Neuroradiological Society. Since 2010 on, the Austrian Society of Neuroradiology, the Belgian Society of Neuroradiology, the British Society of Neuroradiologists, the Bulgarian Association of Radiology, the Czech Neuroradiological Society, the Dutch Society of Neuroradiology, the Finnish Society of Neuroradiology, the German Society of Neuroradiology, the Latvian Society of Neuroradiology, the Norwegian Society of Neuroradiology, the Neuroradiological Section of the Hellenic Radiological Society, the Neuroradiological Section of the Polish Radiological Society, the Portugese Society of Neuroradiology, the Romanian Society of Magnetic Resonance in Medicine, the Spanish Society of Neuroradiology, the Swedish Society of Neuroradiology and the Swiss Society of Neuroradiology decided to choose neuroradiology as their (in some cases second) official Journal.

Submissions of articles can be done via the website: http:/ www.editorialmanager.com/nrad

The Founding Editors of the journal are: M.M. Schechter, New York (deceased) A. Wackenheim, Strasbourg (deceased) S. Wende, Mainz (deceased)

The Editorial Board:

Editor-in-Chief: Guido Wilms (Leuven)

- Section Editor Diagnostic Neuroradiology: Philippe Demaerel (Leuven)

- Section Editor Advanced Imaging: Sven Haller (Geneva)

- Section Editor Head \& Neck Radiology: Jan Casselman (Bruges)

- Section Editor Interventional Neuroradiology: Rudiger von Kummer (Dresden); Editor: Jens Fiehler (Hamburg)

- Section Editor Paediatric Neuroradiology: Andrea Rossi (Genoa)

- Section Editor Japan: Yukunori Korogi (Kitakyushu); Editor: Masaki Komiyama (Osaka)

- $\quad$ Representative ESNR: Nuria Bargalló (Barcelona) 


\title{
SCIENTIFIC PROGRAM
}

\section{"ADVANCED DIAGNOSTIC COURSE TUMOR IMAGING"}

\section{Thursday, September $15^{\text {th }} 2016$}

\author{
Meeting Room 1 \\ Brain Tumors Imaging Update / Moderators: Tatjana Stosic-Opincal, Majda Thurnher \\ 09:00 - 09:30 \\ Brain tumors: Update 2016 what's new \\ 09:30 - 10:00 \\ Biological behavior of astrocytic tumors \\ 10:00 - 10:30 \\ Pretreatment planning of glioma \\ 10:30 - 11:00 Coffee Break \\ From Diagnostic to Treatment / Moderators: Pia Sundgen, Rolf Jaeger \\ 11:00 - 11:30 \\ SWI techniques in brain tumors \\ $11: 30-12: 00$ \\ Perfusion in brain tumors \\ $12: 00-12: 30$ \\ Oncometabolomics MR in brain glioma \\ $12: 30-13: 00$ \\ Posttreatment Imaging in glioma \\ 13:00 - 14:00 Lunch break \\ Differential Diagnostic and Beyond / Moderators: Athanasios Gouliamos, Jan Casselman \\ 14:00 - 14:30 \\ $14: 30-15: 00$ \\ $15: 00-15: 30$ \\ $15: 30-16: 00$ \\ 16:00 - 16:30 Coffee break \\ Tumors-Miscellaneous / Moderators: Andrea Rossi, Katarina Koprivsek \\ $16: 30$ - 17:00 \\ 17:00 - 17:30 \\ $17: 30-18: 00$ \\ 18:00-18:30 \\ Extraaxial tumors \\ MR imaging of cystic lesions of the sellar region \\ Cystic masses \\ Perineural tumor spread \\ Posterior fossa tumors \\ Pediatric brain tumors \\ Spinal cord tumors in children and mimics \\ Spinal bone tumors
}

\author{
Majda Thurnher, Austria \\ Sebastian Brandner, UK \\ Tatjana Stosic-Opincal, Serbia \\ Roberto Gasparotti, Italy \\ Rolf Jager, UK \\ Alberto Beltramello, Italy \\ Pia Sundgren, Sweden
}

Zulejha Merhemic, Bosnia and Herzegovina Fabrice Bonneville, France Alexandre Krainik, France Jan Casselman, Belgium

Friedhelm Zanella, Germany Chen Hoffmann, Israel Andrea Rossi, Italy

Luc van den Hauwe, Belgium 


\section{“ADVANCED COURSE UPDATE ON STROKE MANAGEMENT"}

\section{Meeting Room 2}

Elementary and Advanced Imaging for Patient Selection / Moderators: Rudiger von Kummer, Goran Pavlisa 09:00 - 09:20

09:20 - 09:40

09:40 - 10:00

10:00 - 10:20

10:20 - 11:00 Coffee Break

Fundamentals of recanalization / Moderators: Marko Rados, Tomaz Seruga

11:00 - 11:30

$11: 30-12: 00$

$12: 00-12: 30$

Clot: origin, etiology and imaging

Technical basics of recanalization

Studies: The big 7

$12: 30-13: 00$

New European guidelines

13:00 - 14:00 Lunch Break

Stroke at the Edge / Moderators: Olav Jansen, Istvan Szikora

14:00 - 14:30

New ways in stroke management (stroke networks)

$14: 30-15: 00$

Carotid stenting

15:00 - 15:30

Intracranial stenting

$15: 30-16: 00$

Subclavian and extracranial Vertebral Stenting

16:00 - 16:30 Coffee Break

Venous Infarcts and Diseases / Moderators: Pedro Vilela, Ivan Vukasinovic

16:30 - 16:50

Clinical findings and Imaging of venous thrombosis

$16: 50-17: 20$

$17: 20-17: 40$

$17: 40-18: 00$
Pseudotumor cerebri and venous stenting

AV Fistulas: Classification and Imaging

AV Fistulas: endovascular treatment concepts
Rüdiger von Kummer, Germany

Urs Fischer, Switzerland

Maria Luisa Biscoito, Portugal

Francesco Causin, Italy

Christian Riedel, Germany

Alain Bonafe, France

Wilhelm van Zwam,

Netherland

Jens Fiehler, Germany

Urs Fischer, Switzerland

Olav Jansen, Germany

Istvan Szikora, Hungary

Klaus Hausegger, Austria

Pedro Vilela, Portugal

Jan Gralla, Switzerland

Christoph Cognard, France

Rene Chapot, Germany 
FRIDAY SEPTEMBER $16^{\mathrm{TH}}, 2016$

Meeting Room 1

08:00 - 09:00

Case presentation adults

Diagnostic Vascular I / Moderators: Pia Sundgren, Paulina Due - Tonnessen

09:00 - 09:20

09:20 - 09:40

09:40 - 10:00

10:00 - 10:20

10:30 - 11:00 Coffee Break

Diagnostic Vascular II / Moderators: Naci Kocer, Svetlana Milosevic-Medenica

11:00 - 11:20

$11: 20-11: 40$

$11: 40-12: 00$

12:00 - 14:00 Lunch Break

$12: 00-12: 50$

$13: 10-14: 00$

$14: 00-14: 30$

$14: 30-14: 50$

$14: 50-15: 30$

$15: 30-16: 00$

16:00 - 16:30 Coffee Break

Parallel Scientific Sessions

$16: 30-18: 30$

1-Diagnostic Brain

1-O.1 : Comparison of three different Perfusion CT Software to Predict the Final Infarct after Thrombectomy

Monika Huhndorf

1-O.2 : ASL-based Non-contrast MRDSA with 3D Data Acquisition: Initial Clinical Experience in Intracranial Major Trunk Stenoocclusive Diseases

Kazuhiro Tsuchiya

1-O.3 : Glioma MR Imaging Practices in Europe: Results from the European Society for Neuroradiology (ESNR) Diagnostic Subcommittee Survey on Glioma Imaging

Steffi Thust

1-O.4 : Gadolinium deposition in the brain: 10 years' experience with double dose administration of GBCAs - using a generalized additive model with $\mathbf{R}$

Yae-Won Park

1-O.5 : White matter load and brain atrophy in SLE patients: correlation to cognitive impairment

Björn Cannerfelt
Majda Thurnher

Paulina Due - Tonnessen, Norway

Pia Sundgren, Sweden

Rolf Jager, UK

Beatriz Gomez Anson, Spain

Karl Olof Lovblad, Switzerland Igor Pronin, Russia

Naci Kocer, Turkey
Industry - Sponsored Symposium

Industry - Sponsored Symposium

pening Ceremony

Membership Ceremony

Awards Ceremony

Selected Oral Presentations: Diagnostic Brain 1
1-O.6 : Cognition at baseline before radiotherapy with Temozolomide predict response to treatment in malignant glioma as measured by parametric response maps (PRM)

Pia Sundgren

1-O.7 : Influence of cognitive impairment and depression on the cortical trophism of patients with multiple sclerosis

Emanuele Pravata

1-O.8: McDonald Criteria 2016 revision: Case based MRI evaluation in patients with Clinically Isolated Syndrome

Utku Senol

1-O.9 : Evaluation of T2 Signal Intensity of GH-secreting Pituitary Macroadenoma in Treatment-Naïve Acromegalic Patients Receiving Primary Treatment with Lanreotide Autogel (LAN-ATG) 120 mg Fabrice Bonneville

1-O.10 : Presence of central veins and susceptibility weighted imaging for evaluating lesions in multiple sclerosis and leukoaraiosis Urka Lamot

1-O.11 : Amplitude of low-frequency fluctuations in association with anxiety and depression in subjects with multiple sclerosis: a restingstate fMRI study

Briggith Beltrán 
Meeting Room 2

Psychiatric/Neurodegenerative Disorders / Moderators: Dusko Kozic, Vasilis Katsaros

09:00 -09:20 Imaging of Alzheimer disease

09:20 - 09:40

09:40-10:00

10:30 - 11:00 Coffee Break

\section{Metabolic Diseases/Intoxication / Moderators: Dorith Goldsher, Zoran Rumboldt}

11:00 - 11:20

$11: 20-11: 40$

$11: 40-12: 00$

12:00 - 14:00 Lunch Break

Ceremonies in Meeting

Room 1

16:00 - 16:30 Coffee Break

Parallel Scientific Sessions

16:30 - 18:00

1-Diagnostic Brain

1-O.12 : Correlation between brain volume change and T2 relaxation time in patients with clinically isolated syndrome Alex Rovira

1-O.13 : Safety and diagnostic efficacy of gadoteric acid in magnetic resonance imaging (MRI) of the central nervous system (CNS)

Alex Förster

1-O.14 : The visual evaluation of nigrosome-1 on 3 T susceptibilityweighted imaging may improve diagnostic performance in the patients with Lewy body disease without abnormal findings on 123IMIBG scintigraphy or 99mTc-ECD SPECT

Tomoya Nakatsuka

1-O.15: Brain microstructural changes in patients with pseudoexfoliation syndrome: a tract-based spatial statistics diffusion tensor imaging study.

Anastasia Zikou

1-O.16 : Incomplete hippocampal inversion in patients with focal epilepsy without known etiology and focal MRI abnormalities

$\underline{\text { Raili Raininko }}$
Dusko Kozic, Serbia, Vasilis Katsaros Greece Efrosini Papadaki, Greece

Zoran Rumboldt, Croatia Hrvoje Vavro, Croatia Zoran Rumboldt, Croatia

Selected Oral Presentations: Diagnostic Brain 2

1-O.17 : Treatment effect by advanced MR imaging in adult glioblastoma patients including analysis of regional diffusion variations Anna Rydelius

1-O.18 : Infratentorial superficial siderosis: clinical and radiological spectrum, causes, diagnostic criteria and rational investigation pathway based on an observational case series of 64 patients

$\underline{\text { Peter Cowley }}$

1-O.19 : Diffusion tensor imaging with tract-based spatial statistics reveals widespread microstructural alterations in aspartylglucosaminuria

Anna Tokola

1-O.20 : A Voxel-based morphometry study in diabetes miellitus type II compared to healthy controls. Preliminary results of the PRECISED study.

Alex Rovira

1-O.21 : Redefining the pulvinar sign in fabry's disease: a quantitative mr study

$\underline{\text { Sirio Cocozza }}$

1-O.22 : Improved Detection Rate of Hyperacute Infarction Using Brain CT with Color Look-Up-Table

Kuei-Hong Kuo 


\section{Meeting Room 3}

Interventional Session: Aneurysms / Moderators: Saruhan Cekirge, Rene Chapot

09:00 - 09:20

Flow diverters: what are the indications in cerebral aneurysm

Saruhan Cekirge, Turkey

09:20 - 09:40

What is the role of intrasaccular flow disruptors among other intrasaccular material ?

Laurent Pierot, France

09:40 - 10:00

Did we change our endovascular approach in distal bifurcation aneurysms?

Rene Chapot, Germany

10:00 - 10:20

Giant aneurysms - What is the uptodate treatment?

Naci Kocer, Turkey

10:30 - 11:00 Coffee Break

Interventional Session: AVM / Moderators: Isll Saatci, Jonathan Bhattacharya

11:00 - 11:20

Impact of EVT in cerebral pial AVM

Isıl Saatci, Turkey

$11: 20-11: 40$

Pediatric dural AVFs: Diagnosis,treatment and follow-up

Jonathan Bhattacharya, UK

$11: 40-12: 00$

Adult cerebral dural AVF: Diagnostis, treatment and follow-up

Gyula Gal Denmark

12:00 - 14:00 Lunch Break

Ceremonies in Meeting Room 1

16:00 - 16:30 Coffee Break

Parallel scientific sessions

$16: 30-18: 00$

Selected Oral Presentations: Interventional 1

2-Interventional

2-O.1: Utility of Transbrachial Coil Embolization of Cerebral Aneurysms in the Anterior Cerebral Circulation Takahisa Mori

2-O.2 : Long-Term Outcome of Percutaneous Alcohol Embolization Combined with Percutaneous Vertebroplasty in Aggressive Vertebral Hemangiomas with Epidural Extension Kévin Premat

2-O.3 : Safety and efficacy of antiplatelet response assay and drug adjustment in coil embolization: A propensity score analysis Min Soo Kim

2-O.4 : Inherited transmission of an anatomic conformation favoring aneurismal formation of the anterior communicating artery? Cédric Lenoble

2-O.5 : Endovascular treatment of acute internal carotid artery dissections: technical considerations, clinical and angiographic outcome Wiebke Kurre
2-O.6 : Transvenous coil embolization of cavernous sinus dural arteriovenous fistula on a revised classification

Chao-Bao Luo

2-O.7 : Development of microporous covered stent for the treatment of intracranial aneurysms (NCVC-CS1): its functional features and commencement of investor-initiated clinical trial. Tetsu Satow

2-O.8 : Preinterventional i.v.-rtpa in middle cerebral artery treated with thrombectomy: a double-edged sword?

Johannes Kaesmacher

2-O.9 : Correlation of long term clinical outcome with the degree of collateral circulation in stroke patients treated with stent retrievers Are there indications not to perform thrombectomy based on CT angiography?

Hannes Nordmeyer

2-O.10 : Efficacy of deconstructive method for ruptured vertebral artery dissecting aneurysms Yoji Orita 


\section{SATURDAY SEPTEMBER $17^{\mathrm{TH}}, 2016$}

\section{Meeting Room 1}

08:00 - 09:00

Case presentations interventional

White Matter Diseases / Moderators: Alex Rovira, Dusan Damjanovic

09:00 - 09:20

Multiple sclerosis: Differential diagnosis

09:20 - 09:40

Ultra-high field MRI in Multiple sclerosis

09:40 - 10:00

MRI biomarkers: progressing from qualitative to quantitative image assessment

10:00 - 10:20

Quantitative MRI in Multiple Sclerosis

10:20 - 11:00 Coffee Break

CNS Infections Title /Moderators: Turgut Tali, Ivan Nikolic

11:00 - 11:20

Easy approach to diagnose spinal cord infections

$11: 20-11: 40$

All faces of CNS tuberculosis

$11: 40-12: 00$

Brain Infections Europeans don’t know about

12:00 - 14:00 Lunch Break

12:00 - 12:50

Industry - Sponsored Symposium

\section{ESNR-ESNHR Joint session}

14:00 - 15:00

15:00 - 15:30 Coffee Break

ESNR-ASNR Joint session

ESNR-ESNHR Joint session Title Head and Neck Tumors / Moderator: Sladjana Petrovic,

15:30-15:50

$15: 50-16: 10$

Optic nerve/orbital inflammation

$16: 10-16: 30$

Neoplasm affecting the skull base

Tumors affecting the posterior skull base

$16: 30-16: 50$

Head and Neck Malformations

$16: 50-17: 10$

Advanced imaging in the head and neck When and how

Parallel Scientific Sessions

17:10 - 18:20

Selected Oral Presentations: Interventional 2
Civan Islak/ Naci Kocer

Alex Rovira, Spain

Cristina Auger, Spain

Paul Parizel, Belgium

Tarek Yousry, UK

Turgut Tali, Turkey

Charles Romanowski, UK

Leonardo Macedo, Brazil

Katarina Surlan Popovic, Slovenia Minerva Becker, Switzerland

Christian Czerny, Austria

Athanasios Gouliamos, Greece

Sotirios Bisdas, UK
2-Interventional

2-O.11 : Endovascular treatment of acute basilar artery occlusion: RELOBA study Group experience.

Francesco Biraschi

2-O.12 : Endovascular Treatment of Intracranial Aneurysms Using Acandis Acclino Stents: Mid- and Longterm Results Friedhelm Brassel

2-O.13 : Treatment of ruptured blister aneurysms with flowdiverters: a single centre experience

Chiara Gaudino

2-O.14 : CT Perfusion (CTP) for early detection of vascular complications after Subarachnoid Hemorrage (SH) due to aneurysm rupture. Edoardo Puglielli
2-O.15 : Carotid body tumours-CBT endovascular embolisation using ony $\mathrm{x}$ : single centre experience

Ossama Mansour

2- O.16 : 3-D computational fluid dynamics during mechanical aspiration thrombectomy in acute ischemic stroke

Cecilia Haydee Corzo

2-O.17 : SVS: inter MRI manufacters agreement, inter and intra observers agreement, histo-MR correlation Détraz Lili

2-O.18 : Non-saccular aneurysms of the posterior inferior cerebellar artery: review of a single center series.

Frederic Clarençon 
Meeting Room 2

ESNR-OHBM Joint Session / Moderator: Nuria Bargallo

09:00-09:30

09:30-10:40

10:40 - 11:00 Coffee Break

Special focus: Advance Techniques / Moderator: Kazuhiro Tsuchiya, Japan

11:00 - 11:20 ASL in Alzheimer disease

11:20 - 11:40 fMRI: What Radiologist need to know

11:40 - 12:00 Language lateralization: Other imaging methods

12:00 - 14:00 Lunch Break

Diagnostic Brain Session / Moderator: Dorit Goldsher

14:00 - 14:20

Imaging of the neonatal brain: tips and trics

$14: 20-14: 40$

The short Neuroradiology comicbook

14:40 - 15:00

SYMMETRY in Neuroradilogy-a beauty or challenge

15:00 - 15:30 Coffee Break

ESNR Meets Serbia / Moderators: Ljubo Markovic, Slobodan Cirkovic

$15: 30-15: 50$

Serbian textbook of fetal MRI

$15: 50-16: 10$

$16: 10-16: 30$

$16: 30-16: 50$

16:50 - 17:10

Parallel Scientific Sessions

$17: 10-18: 30$

Selected Oral Presentations: Head \& Neck and Spine
MRI: Imaging the future

Endovascular Treatment of Middle Cerebral Artery Aneurysms

Endovacular treatment of complex intracranial aneurysms
CIS and RR MS
Susan Bookheimer, USA

Paolo Vitali, Cristina Rosazza, Susan Bookheimer

Marion Smits, Netherlands

Cem Calli, Turkey

Sven Haller, Switzerland

Maria Argyropoulou, Greece Martina Spero, Croatia

Dora Zlatareva, Bulgaria

Katarina Koprivsek, Serbia

Dragan Stojanov, Serbia

Milos Lucic, Serbia

Svetlana Milosevic-Medenica, Serbia

Ivan Vukasinovic Serbia
3-Head \& Neck

3-O.1 : Comparsion of contrast and non contrast 3DTOF MR angiography in detecting clinically significant stenosis of ICA(internal carotid artery)

Edin Avdagic

3- O.2 : Use of apparent diffusion coefficients to predict outcome of vestibular schwannomas following Gamma Knife radiosurgery

Chih-Chun Wu

3- O.3 : Plaque morphology and clinical features of symptomatic internal carotid artery stenosis with non-high intensity signal of $\mathrm{T} 1$ weighted magnetic resonance image

Megumu Takata

3- 0.4 : The role of imaging in perineural tumoral spread detection Sladjana Petrovic
4-Spine

4-O.1 : Craniovertebral junction congenital and acquired anomalies Gulgun Yilmaz Ovali

4-O.2 : Integration of Phase Sensitive-Inversion Recovery (PSIR) and Diffusional Kurtosis Imaging (DKI) sequences of the spinal cord $\underline{\text { Valentina Panara }}$

4-O.3 : Imaging of peripheral nerve pathology

Daliborka Marić

4-O.4 : Feasibility of Synthetic MRI for the spine

Maria Isabel Vargas

4-O.5 : Imaging of Spine Fusion: Post Surgery Complications Eduardo Bravo

4-O.6 : The Value of Axial Loading in MRI of Lumbar Spine Deniz Bulja 
5-Pediatric

5-O.1 : Post-mortem High Resolution (HR) magnetic resonance imaging (MRI) in fetuses with brain abnormalities and confirmation of in utero MRI findings

Elisa Scola

5-O.2 : Altered BOLD Signal Following Mirror Therapy on taskbased fMRI in Children with Hemiparetic Spastic Cerebral Palsy

Arzu Ceylan Has

5-O.3 : MRI findings after surgery for congenital heart disease: insight from brain imaging
ARANTXA ROYO

5-O.4 : A practical clinical and imaging approach to pediatric neck masses

Yeliz Pekcevik

5-O.5 : Mapping the fetal brain connectome with high angular resolution diffusion tensor imaging

Andras Jakab

5-O.6 : Dural Arteriovenous Fistulas in children with Vein of Galen Malformation - The role of treatment timing and embolic material Dan Meila

\section{SUNDAY, SEPTEMBER $18^{\mathrm{TH}}, 2016$}

\section{Meeting Room 1}

08:00 - 09:00 Case presentation spine

Pediatric Session: ESNR-ASPNR Joint session / Moderator: Pia Sundgren

09:00 - 09:20 Genetics in metabolic disorders

09:20 - 09:40

Clinical features and imaging findings of inborn errors in metabolism in neonates

09:40 - 10:00

Clinical features and imaging findings of inborn errors in metabolism in childhood

10:00 - 10:20

Metabolic disorders: quiz

10:20 - 10:40

Child abuse imaging

10:40 - 11:00 Coffee Break

\section{Pediatric Session}

$\begin{array}{ll}\text { 11:00 - 11:20 } & \text { Cerebellar vulnerability in prematures } \\ \text { 11:20 - 11:40 } & \text { Overview of neonatal encephalopathies } \\ \text { 11:40 - 12:00 } & \text { Congenital and neonatal infections } \\ \text { 12:00 - 12:20 } & \text { Congenital and neonate tumors }\end{array}$

Meeting Room 2

Spine Diagnostic Session / Moderators: L. Van Den Hauwe, Marek Sasadiak

09:00 - 09:20 Sagittal balance and biomechanics of the spine

09:20 - 09:40 Dynamic evaluation of the spine

09:40 - 10:00 Tractography of the spinal cord: clinical application

10:00 - 10:20 Diagnostic spine evaluation and follow up in hematologic disorders

10:20 - 11:00 Coffee Break

Parallel Scientific Sessions

11:00 - 12:15

Selected Oral Presentations: Diagnostic 3
Johan Van Goethem, Luigi Manfre

Zoltan Patay, USA

Birgit Ertl-Wagner, Germany

Susan Palasis, USA

Bard Nedregaard, Norway

Giulio Zuccoli, USA

\author{
Andrea Rossi, Italy \\ Linda Meiners, Netherlands \\ Tracy Kilborn, South Africa \\ Nadine Girard, France
}

1-Diagnostic

1-O.23 : Mystery of polymicrogyria

Biljana Vukomancic

1-O.24 : Postcontrast STIR Imaging of Intracranial Disease Processes a Pilot Study

Antonija Ruzic Barsic

1-O.25 : Evaluation of changes in the selected white matter tracts in $\mathrm{HCV}$-infected patients using diffusion tensor imaging before and after new interferon-free therapy.

Joanna Bladowska

1-O.26 : Diagnostic Value of Longitudinal Computer-assisted 3D-volumetry in Follow-up MRI of Glioblastoma Georgina Alber
1-O.27 : Anorexia nervosa and MP2RAGE

Maria Isabel Vargas

1-O.28 : Intraoperative resting-state fMRI for lesions related to the sensorimotor area: Technical implementation and preliminary results Ayisha Al Busaidi

1-O.29 : Graph theory analysis of single subject gray-matter structure in malformations of cortical development (MCDs) - a novel approach for diagnostic workup Lajos Rudolf Kozák

12:30 - 12:45 Closing ceremony

12:45 - 13:45 ESNR General Assembly 


\section{ORAL ABSTRACTS}

\section{1 - Diagnostic Brain}

\section{1-0.1}

Category: Diagnostic Brain

Presentation Type : Oral Presentation

Comparison of three different Perfusion CT Software to Predict the Final Infarct after Thrombectomy

Friederike Austein ${ }^{1}, \underline{\text { Monika Huhndorf }}{ }^{1}$, Christian Riedel $^{1}$, Olav Jansen ${ }^{1}$

University Hospital, Schleswig-Holstein Department of Radiology and Neuroradiology Kiel-Germany ${ }^{1}$

Background and Purpose: Our purpose was to determine (1) the accuracy of different commercial perfusion CT-software packages (Philips (A), Siemens (B) and RAPID (C)) to predict the final infarct volume (FIV) after mechanical thrombectomy (MT), and (2) if differences between these packages would alter patient selection for MT based on perfusion imaging criteria.

Methods: Single-institutional CTP data of 147 mechanically recanalized acute ischemic stroke (AIS) patients were post-processed. Ischemic cores were compared to FIV after dividing by TICI-score and by time intervals to reperfusion. FIV was measured at follow-up imaging between days 1-8 after stroke.

Results: In 118 successfully recanalized patients (TICI $2 b / 3$ ), a moderate to strong positive correlation was observed between ischemic core and FIV. The highest accuracy and best correlation are shown in early and fully recanalized patients (Pearson's $r$ for $\mathrm{A}=0.42, \mathrm{~B}=0.64$, and $\mathrm{C}=0.83$; $\mathrm{P}<0.001$ ). BlandAltman plots and boxplots demonstrate that the ranges were smaller in package $\mathrm{C}$ compared to $\mathrm{A}$ and $\mathrm{B}$. Significant differences were found between the packages regarding over- and underestimation of ischemic core. Package A, compared to $\mathrm{B}$ and $\mathrm{C}$, estimated more than twice as many patients with a malignant stroke profile $(\mathrm{P}<0.001)$. Package $\mathrm{C}$ best predicted hypoperfusion volume in nonsuccessful recanalized patients.

Conclusions: Our study demonstrates best accuracy and approximation between the results of a fully automated software (RAPID) and FIV, especially in early and fully recanalized patients. Further, this software package significantly less overestimated the FIV and less often estimated a malignant mismatch profile compared to other software.

\section{1-0.2}

Category: Diagnostic Brain

Presentation Type : Oral Presentation

Keywords: brain, MRA, ASL

\section{ASL-based Non-contrast MRDSA with 3D Data Acquisition: Initial Clinical Experience in Intracranial Major Trunk Stenoocclusive Diseases}

Kazuhiro Tsuchiya $^{1}$, Miho Gomyo $^{2}$, Arisa Ohara ${ }^{2}$, Shichiro Katase ${ }^{2}$, Isao Miyazaki

Tokyo Teishin Hospital Department of Radiology Tokyo-Japan ${ }^{1}$ Kyorin University Department of Radiology Tokyo-Japan ${ }^{2}$ Kyorin University Radiology Department Tokyo-Japan ${ }^{3}$

Purpose: Arterial spin labeling (ASL) has been applied to visualize cerebral arteries as non-contrast MRDSA. We have developed an ASL-based MRDSA sequence that acquires 3D signal data at multiple interval times (multi-TI MRA with signal targeting alternating radiofrequency using asymmetric inversion slab ASL technique: mASTAR). Our purpose was to assess its feasibility in the diagnosis of stenoocclusion of major intracranial arteries.

Materials and Methods: Six-phase 3D MRDSA was performed using a 3D-FFE sequence with an initial delay of $435 \mathrm{~ms}$ followed by 5 phases with a $235-\mathrm{ms}$ interval. In 5 volunteers, we compared the CNR between mASTAR and 3D-TOF MRA at ICA trunk and two portions of the MCA. In 22 patients with stenosis/occlusion of the MCA and ICA including those with moyamoya disease as well as those after bypass surgery, we visually scored demonstration of the lesion and that of branches distal to the lesion and bypass recipient vessels between the two methods.

Results: 3D MRDSA by mASTAR yielded six-phase serial images in $5 \mathrm{~min} 17 \mathrm{sec}$. In the CNR assessment, mASTAR was inferior to 3DTOF MRA at the 3 portions. In the patient group, however, as for demonstration of stenosis, the two techniques well corresponded in 21 patients, while discrepancy was noted in 1 patient. Additionally, mASTAR better depicted distal branches in 15 patients, while two methods were equivalent in the remaining 7 patients.

Conclusion: In cerebral major artery stenoocclusive lesions, 3D MRDSA by the mASTAR technique could provide images reflecting hemodynamics in an acceptable scanning time.

\section{1-O.3}

Category: Diagnostic Brain

Presentation Type : Oral Presentation

Glioma MR Imaging Practices in Europe: Results from the European Society for Neuroradiology (ESNR) Diagnostic Subcommittee Survey on Glioma Imaging

$\underline{\text { Steffi Thust }}^{1}{ }_{-}$, Tarek Yousry ${ }^{1}$, Nuria Bargallo ${ }^{2}$, Meike Vernoij ${ }^{3}$, Marion Smits $^{3}$

National Hospital for Neurology and Neurosurgery Lysholm Department of Neuroradiology London-United Kingdom ${ }^{1}$ Hospital Clinic i Provincial Servicio de Neurorradiologica Barcelona-Spain ${ }^{2}$ Erasmus MC Neuroradiology Rotterdam-The Netherlands ${ }^{3}$

Purpose: To assess current glioma imaging practices throughout Europe. The information gained from this survey will form the basis for the publication of practical MRI recommendations.

Method: Survey invitations were emailed to ESNR members ( $\mathrm{n}=1662)$ and known associates ( $\mathrm{n}=6400)$, European national radiologists' societies and distributed via social media. To avoid duplicate bias, participants were instructed to supply institution details or confirm they were the only person answering from their centre. The questionnaire featured 87 items, divided into multiple choice, single best choice and free text questions on personal practice and preferred MRI techniques.

Results: 227 individuals (59.7\% academic centres, 37.2\% general hospitals) participated. $80.2 \%$ were neuroradiologists, $9.3 \%$ general radiologists, $6.2 \%$ trainees and $4.4 \%$ other professionals. Conventional MRI: $99.1 \%$ used DWI and $82.4 \%$ performed $3 \mathrm{D}$ sequences (T1 $>$ FLAIR $>$ T2). Advanced MRI: $85.5 \%$ employed perfusion for grading and/or follow up, with $48.5 \%$ acquiring it universally. $81.0 \%$ used MRS to characterise lesions, but rarely routinely $(21.2 \%) .49 .1 \%$ performed fMRI and $63.7 \%$ DTI, with approximately $60 \%$ results integrated into navigation systems. Reasons for omitting advanced modalities included lack of facility/software, time constraints and no requests. Clinical scenarios: $23.0 \%$ participants used a reporting template, $60.4 \%$ issued qualitative reports, $27.6 \%$ obtained RANO measurements. Postoperative MRI was routinely carried out by $63.1 \%$ within $24-72 \mathrm{hrs}$ with $17.2 \%$ reporting a percent measure of resection.

Conclusion: Glioma imaging standards must reflect best practice, but consider differences of equipment, levels of experience, financial and healthcare economic factors. This survey has yielded valuable 
information on current practices, controversies and potential technical hurdles to the implementation of state-of-the-art techniques.

\section{1-O.4}

Category: Diagnostic Brain

Presentation Type : Oral Presentation

Keywords : Gadolinium deposition, GBCA, dentate nucleus, linear, macrocyclic

Gadolinium deposition in the brain: 10 years' experience with double dose administration of GBCAs - using a generalized additive model with $\mathbf{R}$

Sohi Bae ${ }^{1}$, Yae-Won Park ${ }^{1}$, Ho-Joon Lee ${ }^{1}$, Yoon Seong Choi ${ }^{1}$, Jinna Kim ${ }^{2}$, Seung-Koo Lee ${ }^{1}$

Yonsei University College of Medicine Radiology Seoul-Korea, South ${ }^{1}$

Purpose: To evaluate the effect of administration of various gadoliniumbased contrast agents (GBCAs) on the T1 hyperintensity in the dentate nucleus (DN) and globus pallidus (GP) on magnetic resonance (MR) images

Materials and Methods: The study included 172 patients who underwent more than ten instances of double-dose GBCA-enhanced MR during the last 10 years. Two radiologists conducted a quantitative analysis of unenhanced T1-weighted images by using regions of interest. The GPto-thalamus $(\mathrm{TH})$ signal intensity ratio, DN-to-pons signal intensity ratios, and relative percentage change (Rchange) between the first and last examinations were calculated. The relationships between the Rchange and several factors, including the number of GBCA administrations, were analyzed by using a generalized additive model. Interobserver agreement was evaluated using the Lin concordance correlation coefficient.

Results: Patients were administered various GBCAs, with a mean of $6.9 \pm$ 6.9 (standard deviation) doses of gadodiamide, $6.4 \pm 2.6$ of gadopentetate dimeglumine, $10.4 \pm 12.7$ of gadobutrol, $6.2 \pm 4.9$ of gadoterate meglumine, and $0.4 \pm 0.1$ of gadoxetate disodium. Rchange for the $\mathrm{DN}$ :pons ratio was associated with gadodiamide $(\mathrm{p}=0.009)$ and gadopentetate dimeglumine $(\mathrm{p}<0.001)$, though not with other GBCAs. Rchange for the GP:TH ratio was not associated with the GBCA administrations. Interobserver agreement was almost perfect $(0.99 ; 95 \%$ confidence interval: 0.99, 0.99).

Conclusion: Previous administrations of multiple doses of macrocyclic GBCAs are not associated with $\mathrm{T} 1$ hyperintensity in the $\mathrm{DN}$, while linear GBCAs show such association.

\section{1-0.5}

Category: Diagnostic Brain

Presentation Type : Oral Presentation

Keywords : SLE, MRI, white matter load, brain atrophy, cognitive dysfunction

\section{White matter load and brain atrophy in SLE patients: correlation to cognitive impairment}

Björn Cannerfelt ${ }^{1}$, Jessika Nystedt ${ }^{2}$, Jimmy Lätt ${ }^{1}$, Andreas Jönsen ${ }^{3}$,

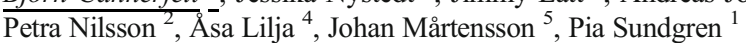

Lund University Radiology Lund-Sweden ${ }^{1}$ Lund University Neurology Lund-Sweden ${ }^{2}$ Lund University Reumatology Lund-Sweden ${ }^{3}$ Lund University Center for Primary Care Lund-Sweden ${ }^{4}$ Lund University Psychology Lund-Sweden ${ }^{5}$

Background: Cognitive dysfunction and brain abnormalities is common in Neuropsychiatric SLE (NPSLE). However, distinguishing NPSLE and
non-NPSLE is problematic since both might display cognitive dysfunction and brain abnormalities.

Purpose: To evaluate white matter load and brain atrophy in a cohort of SLE patients and correlate findings to cognitive dysfunction.

Material and Methods: 70 SLE patients (43 NPSLE, 27 nonNPSLE) and 25 Healthy Controls underwent clinical examination, a $3 \mathrm{~T}$ brain MRI and a standardized cognitive test (CNS Vital Signs $^{\mathrm{TM}}$ ). MRI data was analyzed for white matter load and brain atrophy using two automated post processing programs; Lesion Segmentation Tool and FreeSurfer respectively. White matter load and brain atrophy were calculated and differences between groups and subgroups were evaluated and correlated to cognitive dysfunction.

Results: The volume and the number of white matter lesions was significantly higher in NPSLE and non-NPSLE compared to HC $(\mathrm{p}<0.05)$. Atrophy of the hippocampus was significantly higher in NPSLE compared to non-NPSLE. A negative correlation existed between scores on cognitive test domains: composite memory $(p=0,032)$ and verbal memory $(p=0,008)$ and white matter lesion volume.

Conclusion: This study further indicates that patients with SLE has a significantly higher volume of white matter lesions on MRI compared to $\mathrm{HC}$ and the volume of these lesions correlates to the patients cognitive dysfunction. Furthermore, atrophy of the hippocampus is more pronounced in NPSLE compared non-NPSLE.

\section{1-0.6}

Category: Diagnostic Brain

Presentation Type : Oral Presentation

Keywords : cognition, malignant gliomas, parametric response maps, response to treatment

Cognition at baseline before radiotherapy with Temozolomide predict response to treatment in malignant glioma as measured by parametric response maps (PRM)

Åsa Lilja ${ }^{1}$, Anna Rydelius ${ }^{2}$, Björn Lampinen ${ }^{1}$, Johan Bengzon ${ }^{3}$, Silke Engelholm ${ }^{4}$, Sara Kinhult ${ }^{4}$, Pia Sundgren ${ }^{1}$

Radiology Clinical Sciences Lund-Sweden ${ }^{1}$ Neurology/Radiology Clinical Sciences Lund-Sweden ${ }^{2}$ Neurosurgery Clinical Sciences Lund-Sweden ${ }^{3}$ Oncology Clinical Sciences Lund-Sweden ${ }^{4}$

Only a few studies have been concerned with cognitive functioning in relation to survival in patients with malignant brain tumors. Executive function, attention and verbal memory are the cognitive domains most closely associated with poor prognosis [1]. Thirty three patents were cognitively assessed at baseline before postoperative treatment, at 3 months and at 1 year. Five out of these 33 patients were also subjects in a pilot parametric response map (PRM) study. Already at three weeks after postoperative therapy start 2 out of the 5 patients were classified as having stable disease/ partial response (SD) and 3 patients were classified as having progressive disease (PD) using PRM, verified at 8 months using conventional MRI. Evaluation of cognitive functioning of all patients was performed independently. The 3 patients with subnormal cognitive results already at pretreatment assessments were the same 3 patients that were classified as non-responders/ progressive disease (PD). The 2 patients with normal cognitive results were classified as having stable disease (SD). At follow up 3 months after treatment all cognitive results were essentially unchanged compared to pre-treatment results.

1. Johnson, D.R., et al., Early measures of cognitive function predict survival in patients with newly diagnosed glioblastoma. NeuroOncology, 2012. 14(6): p. 808-816. 
1-0.7

Category: Diagnostic Brain

Presentation Type : Oral Presentation

Influence of cognitive impairment and depression on the cortical trophism of patients with multiple sclerosis

Emanuele Pravata ${ }^{1}$, Mara Rocca ${ }^{2}$, Paola Valsasina ${ }^{2}$, Gianna Riccitelli ${ }^{2}$, Claudio Gobbi ${ }^{3}$, Giancarlo Comi ${ }^{4}$, Andrea Falini ${ }^{5}$, Massimo Filippi ${ }^{4}$

Neurocenter of Southern Switzerland Neuroradiology Dep. LuganoSwitzerland ${ }^{1}$ San Raffaele Scientific Institute, Vita-Salute San Raffaele University Neuroimaging Research Unit, Institute of Experimental Neurology Milano-Italy ${ }^{2}$ Neurocenter of Southern Switzerland Neurology Dep. -Switzerland ${ }^{3}$ Raffaele University Department of Neurology Milano-Italy ${ }^{4}$ Raffaele University Department of Neuroradiology Milano-Italy ${ }^{5}$

Purpose: To investigate gray matter (GM) trophism in multiple sclerosis (MS) patients with cognitive impairment and depression using cortical thickness analysis.

Methods: 3.0T brain high-resolution 3D T1-weighted scans were obtained from $126 \mathrm{MS}$ patients and 59 matched healthy controls. Cognitive assessment was performed using the Brief Repeatable Battery of Neuropsychological Tests. Patients with at least two abnormal tests were considered cognitively impaired (CI). Depression was assessed using the Montgomery-Asberg Depression Rating Scale. Patients were classified as depressed (D) if their score was $>9$.

Cortical thickness was assessed with FreeSurfer 5.3. A vertex-byvertex analysis assessed differences of cortical thickness between controls and MS patients, and among patients CI / non-CI and D / non-DI subgroups. A conjunction analyses tested overlapping effects of CI and D.

Results: Compared with controls, MS patients exhibited widespread bilateral cortical thinning involving all brain lobes. CI MS patients showed cortical thinning particularly in the frontal, temporal and parietal lobes. D MS patients showed areas of both thinning and thickening in the orbitofrontal and dorsolateral prefrontal cortex. A conjunct effect of depression and cognitive impairment was seen in the ventral orbitofrontal and anterior temporal cortex.

Conclusions: Cognitive impairment in MS patients is linked to cortical thinning in several areas involved in executive control, working memory, and problem solving. Remarkably, D MS patients exhibited both reduction and increase of the GM trophism in areas involved in mood and visceromotor control integration. A conjunct effect of $\mathrm{D}$ and $\mathrm{CI}$ was seen in areas deputed to emotional behaviour and self-monitoring.

\section{1-0.8}

Category: Diagnostic Brain

Presentation Type : Oral Presentation

Keywords : Multipl Sclerosis, Magnetic Resonance Imaging

McDonald Criteria 2016 revision: Case based MRI evaluation in patients with Clinically Isolated Syndrome

$\underline{\text { Utku Senol }}^{1}$, Alp Öztek ${ }^{2}$, Ali Dablan ${ }^{2}$, Ferah Kızılay ${ }^{3}$, Kamil Karaali ${ }^{2}$

Akdeniz University Neuroradiology Antalya-Turkey ${ }^{1}$ Akdeniz University Radiology Antalya-Turkey ${ }^{2}$ Akdeniz University Neurology Antalya-Turkey ${ }^{3}$

The purpose of this presentation is to discuss DIS and DIT findings that should be included in MRI reports of MS patients based on the recently revised McDonald criteria.
Multiple sclerosis is the most common chronic neurological disease of young adults. Since clinical presentation can be complicated in these patients, MRI has a critical role in diagnosis. McDonald criteria are especially important to differentiate between clinically isolated syndrome and clinically definite multiple sclerosis (CDMS). The criteria, which are used to diagnose MS, have been revised since 2001, with the final revision having been made in 2016. The major difference from 2010 is that the number of required periventricular lesions for DIT positivity is 3 and optic neuritis is also considered as a characteristic location for purposes of DIT positivity.

In this educational presentation, we present six different cases referred to our department as clinically isolated syndrome, with findings covering recently revised McDonald criteria. After MRI, all cases were diagnosed CDMS. Two of these cases had applied after their first episode. In conclusion, MRI reports are essential for CDMS diagnosis. Studies are underway to standardise clinical information in the radiology request forms, MRI image acquisition, reports and frequency of follow-up of patients with MS. There are similar works to standardise radiology reports, and it is recommended to include DIS and DIT findings in the reports.

1-0.9

Category: Diagnostic Brain

Presentation Type : Oral Presentation

Keywords : Pituitary adenoma - acromegaly - MRI T2 signal intensity somatostatin analogue

Evaluation of T2 Signal Intensity of GH-secreting Pituitary Macroadenoma in Treatment-Naïve Acromegalic Patients Receiving Primary Treatment with Lanreotide Autogel (LAN-ATG) $120 \mathrm{mg}$

Fabrice Bonneville ${ }^{1}$, Louis-David Riviere ${ }^{1}$, Aude Houchard ${ }^{2}$, Caroline Sert $^{2}$, Philippe Caron ${ }^{3}$

Toulouse University Hospital Neuroradiology Toulouse-France ${ }^{1}$ Ipsen Statistics Boulogne-France ${ }^{2}$ Toulouse University Hospital Endocrinology Toulouse-France ${ }^{3}$

Introduction: T2-signal intensity of GH-secreting pituitary adenomas may be associated with the response to somatostatin analogue therapy in acromegalic patients. Here, we explore how best to evaluate T2-signal intensity of GH-secreting pituitary macroadenoma.

Methods: T2-signal intensity from 85 treatment-naïve acromegalic patients with GH-secreting pituitary macroadenoma receiving monthly LAN-ATG $120 \mathrm{mg}$ were evaluated at baseline and during treatment. T2-signal intensity was defined as hypo-/iso-/hyper-intense based on comparison of adenoma/normal cerebral parenchyma, according to a qualitative method (visually comparing tissues); and two quantitative methods using ratio of signal intensities of regionof-interests in either tissues (adenoma vs grey matter only [first method] or vs grey matter and white matters in adjacent temporal lobe [second method]). Here, we present the results of baseline MRI evaluations according 3 methods.

Results: A greater proportion of patients' macroadenomas were categorized as hypointense using the qualitative method (59\%) than the quantitiative methods ( $36 \%$ and $20 \%$ ). In addition, more of the patients showing full hormonal control or tumoural response had hypointense macroadenoma using the qualitative than using the quantitative methods. Conclusion: A simple qualitative method could be used to identify MRI T2-signal hypointensity in treatment-naïve acromegalic patients with GH-secreting pituitary macroadenoma, which could be associated with later favourable responses to LAN-ATG primary therapy 
1-0.10

Category: Diagnostic Brain

Presentation Type : Oral Presentation

Keywords : multiple sclerosis, susceptibility weighted imaging, central vein

Presence of central veins and susceptibility weighted imaging for evaluating lesions in multiple sclerosis and leukoaraiosis

$\frac{\text { Urška Lamot }}{{ }^{1}}{ }^{\text {, }}$, Katarina Surlan Popovič ${ }^{1}$, Jernej Avsenik ${ }^{1}$,

University Medical Centre Ljubljana Clinical Institute of Radiology Ljubljana-Slovenia ${ }^{1}$

Purpose: The process of demyelination in multiple sclerosis (MS) is reflected in lesions of the central nervous system, which are also found in an abundance of other diseases and are radiographically indistinguishable. Susceptibility weighted imaging (SWI) may have the potential to depict the centrally running veins within MS lesions, which is presumed to be a specific finding for MS.

Methods and Materials: We retrospectively examined 34 subjects with MS and 19 subjects with ischaemic lesions, which underwent a 3 T MRI investigation. According to FLAIR and T2-weighted images the lesions were categorized regarding location: supratentorial peripheral, supratentorial periventricular, or infratentorial. The presence of central veins $(\mathrm{CVs})$ was determined on SWI. Gadolinium enhanced T1weighted images were included for the evaluation of active lesions in patients with MS.

Results: A total load of 601 MS and 204 ischaemic lesions was identified and we found significantly more lesions with CVs in the group with ischaemic lesions compared to the group with MS lesions $(p<0.001)$. Similarly significantly more supratentorial peripheral ischaemic lesions had a CV $(\mathrm{p}=0.011)$. For the supratentorial periventricular and intratentorial lesions there was no significant difference between $\mathrm{CV}$ s in MS and ischaemic lesions $(\mathrm{p}=0.377$ and $\mathrm{p}=0.615)$. Comparing the active and inactive MS lesions regarding CVs, we found no significant difference between the two groups $(\mathrm{p}=0.472)$.

Conclusion: We can conclude that $\mathrm{CVs}$ are not a specific radiographic diagnostic sign for MS, nonetheless we can use the presence of CVs to exclude some other potential causes of the lesions in the central nervous system.

\section{1-O.11}

Category: Diagnostic Brain

Presentation Type : Oral Presentation

Keywords : Magnetic resonance; Brain; Pseudoatrophy; Clinically isolated syndrome

Correlation between brain volume change and $\mathrm{T} 2$ relaxation time in patients with clinically isolated syndrome

Alex Rovira ${ }^{1}$, Cristina Auger ${ }^{1}$, Manel Alberich ${ }^{1}$, Deborah Pareto ${ }^{1}$, Jaume Sastre-Garriga $^{2}$, Mar Tintoré ${ }^{2}$, Xavier Montalban ${ }^{2}$, Francesc Xavier Aymerich ${ }^{1}$

Hospital Vall Hebron Section of Neuroradiology and MR Unit BarcelonaSpain ${ }^{1}$ Hospital Vall Hebron CEMCAT Barcelona-Spain ${ }^{2}$

Background and Objective: Regional volumetric studies have suggested that reversible brain volume changes (pseudoatrophy) are mostly confined to the white matter. The aim of this study is to correlate pseudo$\mathrm{T} 2$ values (a marker of hydration status) with brain volume changes in patients with clinically isolated syndrome (CIS).
Materials and Methods: 96 patients with CIS were included (62 women; median age, 33 years; age range, [19, 49]; EDSS median, 2; EDSS range, $[0,4.5]$; mean disease duration, 3.78 months). Baseline and 12 months dual echo T2-weighted, and 3D T1-weighted (MPRAGE) sequences were acquired on a 3.0T. The dual-echo sequence was used to produce pseudo-T2 maps (pT2). Pseudo-T2 values were evaluated in regions of normal appearing white matter. MPRAGE sequence was used to obtain white and grey matter volumetric fractions. Changes between month 12 and baseline studies in pT2, white and grey matter fractions were evaluated by the Spearman rank correlation.

Results: A significant, although weak positive correlation $(\mathrm{r}=0.276$, $\mathrm{p}=0.006$ ) between changes in pT2 and changes in white matter was found. Changes in grey matter did not correlate with changes in pT2 $(\mathrm{r}=-0.002, \mathrm{p}=0.982)$.

Conclusions: The results suggest that white matter volume changes in patients with multiple sclerosis can be partially explained by fluctuations in brain water. They also support the value of pseudo-T2 measures to assess white matter water changes, and its potential role in distinguishing reversible (pseudoatrophy) from irreversible (atrophy) brain tissue loss.

\section{1-O.12}

Category: Diagnostic Brain

Presentation Type : Oral Presentation

\section{Keywords : MS}

Amplitude of low-frequency fluctuations in association with anxiety and depression in subjects with multiple sclerosis: a resting-state fMRI study

Briggith Beltrán ${ }^{1}$, Gerard Blasco ${ }^{1}$, Carles Biarnes ${ }^{1}$, Jordi Gich ${ }^{2}$, Mireia Rivero $^{2}$, Jüdith Salvedra ${ }^{2}$, Pepus Daunis-i-Estadella ${ }^{3}$, Salvador Pedraza $^{1}$, Lluís Ramió ${ }^{2}$, Puig Josep ${ }^{1}$

Imaging Research Unit. Diagnostic Imaging Institiute (IDI). Dr. Josep Trueta University Hospital Radiology Girona-Spain ${ }^{1}$ Neuroimmunology and Multiple Sclerosis Unit, Department of Neurology, Dr. Josep Trueta University Hospital Neurology Girona-Spain ${ }^{2}$ University of Girona Computer Science, Applied Mathematics and Statistics Girona-Spain ${ }^{3}$

Purpose: To investigate differences in amplitude of low-frequency fluctuations (ALFF) in resting state functional magnetic resonance imaging (rs-fMRI) among cohorts of subjects with multiple sclerosis (MS) stratified by progression and to evaluate ALFF's relation to anxiety and depression.

Methods: We prospectively studied consecutive patients with radiologically isolated syndrome or clinically isolated syndrome $(\mathrm{n}=23)$, primary or secondary progressive (PP/PS) $(n=35)$, relapsing-remitting $(n=24)$ multiple sclerosis and 30 healthy controls on a $1.5 \mathrm{~T}$ scanner. Imaging included 10 minutes of rs-fMRI acquisition and anatomic T1 TFE. We used the Hospital Anxiety and Depression (HAD) scale. To avoid confounding effects of age or schooling on cognitive performance, we used unstandardized residual values of the regression between cognitive scores and these factors for all analyses. We used analysis of variance and Bonferroni test to assess differences between groups. To analyze the impact of ALFF on HAD score among groups, we used analysis of covariance (ANCOVA) on variables associated with cognitive scores $(\mathrm{p}<0.05)$.

Results: HAD-depression subscale differed between PP/PS and controls $(p<0.018)$; no other differences in HAD scores were found between study groups. Differences in HAD-anxiety scores among groups were explained by mean ALFF in the left posterior cingulate gyrus $(\mathrm{p}=0.004)$. The best ANCOVA model to explain HAD-depression scores among groups $(p<0.001)$ included mean ALFF in the left mid cingulate 
$(\mathrm{p}=0.029)$, left inferior frontal gyrus $(\mathrm{p}=0.011)$, left medial frontal gyrus $(\mathrm{p}=0.001)$, and right superior temporal gyrus $(\mathrm{p}=0.033)$.

Conclusion: Our preliminary results suggest ALFF on rs-fMRI is associated with anxiety and depression scores in subjects with MS.

\section{$1-0.13$ \\ Category: Diagnostic Brain \\ Presentation Type : Oral Presentation}

Keywords : gadoteric acid, CNS, MRI, safety, efficacy

Safety and diagnostic efficacy of gadoteric acid in magnetic resonance imaging (MRI) of the central nervous system (CNS)

Alex Förster $^{1}{ }_{-}$, Oliver Heine ${ }^{2}$, Michael Wolf ${ }^{3}$, Christoph Groden ${ }^{1}$

Universitätsmedizin Mannheim Neuroradiology Mannheim-Germany ${ }^{1}$ Guerbet GmbH Sulzbach-Germany ${ }^{2}$ mwi Püttlingen-Germany ${ }^{3}$

Purpose: To assess the safety and diagnostic efficacy of gadoteric acid (Dotarem ${ }^{\circledR}$, Guerbet, France) in MRI examinations of the brain, spinal cord, and surrounding tissue.

Material and Methods: As part of a post-marketing surveillance study conducted in 52 centres between 2011 and 2013, 21,959 neuroradiological examinations $(82 \%$ of the brain, $20 \%$ of the spinal cord, $4 \%$ of surrounding tissue [including multiple examinations]) were evaluated for safety (adverse events) and efficacy (diagnostic value [yes/no] and image quality [5-step scale from very poor to very good]).

Results: The patients ( $58.2 \%$ female) had a mean age of $52.8 \pm 17.2$ years and included 42 children under 12 years of age and 204 adolescents aged $12-17$ years. At least one risk factor was reported in $26.0 \%$ of patients, mainly allergies (15.6\%) and hypertension (6.1\%). Adverse events (AEs) occurred in 64 patients $(0.3 \%)$, including 2 adolescents. In 3 cases $(0.01 \%)$, the AEs were serious: 1 adolescent (swelling face, urticaria), 2 adults (allergy and renal failure for one, eye pruritus, cough, nasal congestion, sneezing for the other). All AEs fully resolved. Image quality was good or very good in $99.0 \%$ of patients. It was possible to make a diagnosis in $100 \%$ of children and adolescents and in $99.8 \%$ of adults.

Conclusion: Gadoteric acid has proved to be a safe MRI contrast agent in neuroradiology, with high diagnostic value for CNS examinations.

\section{1-0.14}

Category: Diagnostic Brain

Presentation Type : Oral Presentation

Keywords : susceptibility-weighted imaging, Lewy body disease

The visual evaluation of nigrosome-1 on 3T susceptibility-weighted imaging may improve diagnostic performance in the patients with Lewy body disease without abnormal findings on 123I-MIBG scintigraphy or 99mTc-ECD SPECT

Tomoya Nakatsuka ${ }^{1}$, Shusuke Kasuya ${ }^{1}$, Masayuki Odashima ${ }^{1}$, Noriko Kitamura ${ }^{1}$, Tsutomu Inaoka ${ }^{1}$, Hitoshi Terada ${ }^{1}$

Toho University Sakura Medical Center Radiology Sakura-Japan ${ }^{1}$

Purpose: To determine whether the visual evaluation of nigrosome-1 on 3T susceptibility-weighted imaging (SWI) improves diagnostic performance in the patients with Lewy body disease (LBD) without abnormal findings on ${ }^{123}$ I-FP-CIT SPECT, ${ }^{123}$ I-MIBG scintigraphy or ${ }^{99 m}$ Tc-ECD SPECT.

Materials and Methods: We retrospectively reviewed 26 patients with LBD who had undergone ${ }^{123} \mathrm{I}$-FP-CIT SPECT, ${ }^{123} \mathrm{I}$-MIBG scintigraphy, ${ }^{99 \mathrm{~m}} \mathrm{Tc}$ -
ECD SPECT and 3T SWI to evaluate nigrosome-1 all for detailed examination of LBD. Bilateral striatal accumulation of ${ }^{123}$ I-FP-CIT was measured as the specific binding ratio. Cardiac uptake of ${ }^{123} \mathrm{I}-\mathrm{MIBG}$ was measured as the heart-to-mediastinum ratio in the delayed phase. Occipital hypoperfusion on ${ }^{99 \mathrm{~m}}$ Tc-ECD SPECT was assessed using an eZIS. McNemar's tests were performed to evaluate the usefulness of adding 3T SWI.

Results: ${ }^{123}$ I-FP-CIT SPECT showed abnormal striatal accumulation in 24 patients. ${ }^{123}$ I-MIBG scintigraphy showed abnormal cardiac uptake in 19 patients. ${ }^{99 \mathrm{~m}} \mathrm{Tc}-\mathrm{ECD}$ SPECT showed significant occipital hypoperfusion in 12 patients. Nigrosomal signal abnormality on 3T SWI was detected in one out of 2 patients with normal striatal accumulation on ${ }^{123} \mathrm{I}$ FP-CIT SPECT ( $\mathrm{p}=0.32$, NS), 6 out of 7 patients with normal cardiac uptake on ${ }^{123} \mathrm{I}$-MIBG scintigraphy $(\mathrm{p}=0.01<0.05)$ and 13 out of $14 \mathrm{pa-}$ tients without significant occipital hypoperfusion on ${ }^{99 \mathrm{~m}} \mathrm{Tc}-\mathrm{ECD}$ SPECT $(\mathrm{p}=0.0003<0.05)$.

Conclusion: The visual evaluation of nigrosome-1 on 3T SWI may improve diagnostic performance in the patients with LBD without abnormal findings on ${ }^{123} \mathrm{I}-\mathrm{MIBG}$ scintigraphy or ${ }^{99 \mathrm{~m}} \mathrm{Tc}-\mathrm{ECD}$ SPECT.

\section{1-0.15}

Category: Diagnostic Brain

Presentation Type : Oral Presentation

Keywords : Magnetic resonance imaging; pseudoexfoliation syndrome; diffusion tensor imaging; tract-based spatial statistics; white matter hyperintensities.

Brain microstructural changes in patients with pseudoexfoliation syndrome: a tract-based spatial statistics diffusion tensor imaging study.

Anastasia Zikou $^{1}{ }^{\text {, George Kitsos }}{ }^{2}$, Loukas Astrakas ${ }^{3}$, Chrysavgi Pappa ${ }^{4}$, Maria Argyropoulou ${ }^{1}$

Medical School,University of Ioannina Radiology Ioannina-Greece ${ }^{1}$ Medical School,University of Ioannina Ophthalmology IoanninaGreece ${ }^{2}$ Medical School,University of Ioannina Medical Physics Ioannina-Greece ${ }^{3}$ University Hospital, Ioannina Ophthalmology Ioannina-Greece ${ }^{4}$

Background and Purpose: There is evidence of chronic cerebral hypoperfusion and cerebrovascular disease in pseudoexfoliation syndrome (PXS). The purpose of the present study was to evaluate structural brain changes in patients with PXS by assessing white mater hyperintensities (WMHIs) and by using the tract-based spatial statistics (TBSS) method of diffusion tensor imaging (DTI) analysis.

Material and Methods: Eighteen PXS patients (mean age \pm SD; $62.75 \pm 0.4$ years) and 18 control subjects (mean age $\pm \mathrm{SD} ; 62 \pm 0.6$ years) underwent brain magnetic resonance imaging and complete ophthalmological examination. The imaging protocol consisted of sagittal fluid attenuated inversion recovery sequence and a spin-echo, echo-planar diffusion-weighted sequence. Results: WMHIs were observed in 14 of the 18 patients with PXS (77.7\%) and in 8 of the 18 control subjects $(44.4 \%)(\mathrm{P}=0.42)$. A significantly larger number of WMHIs was found in the patients with PXS (median: 6, range: $0-29)$ than in the control subjects (median: 0.5 , range: $0-15)(P=0.002)$. TBSS showed increased MD, $\mathrm{AD}$ and $\mathrm{RD}$ values in the following white matter tracts bilaterally: superior longitudinal fasciculus, inferior longitudinal fasciculus, corticospinal tract, anterior thalamic radiation, inferior fronto-occipital fasciculus, cingulum, uncinate fasciculus, and forceps minor in PXS patients compared to control subjects. No significant differences were observed in FA values between patients and control subjects.

Conclusion: Patients with PXS showed increased WMHIs probably related to chronic cerebral hypoperfusion. The increased $\mathrm{MD}, \mathrm{AD}$ and $\mathrm{RD}$ values in major white matter tracts may be a secondary phenomenon, representing demyelination and axonal degeneration. 
1-0.16

Category: Diagnostic Brain

Presentation Type : Oral Presentation

Keywords : Hippocampus Epilpepsy Volumetry MRI Morphology

Incomplete hippocampal inversion in patients with focal epilepsy without known etiology and focal MRI abnormalities

Dragan Dajic ${ }^{1}$, Eva Kumlien ${ }^{2}$, Raili Raininko ${ }_{-}^{1}$

Uppsala university Radiology Uppsala-Sweden ${ }^{1}$ Uppsala university Neurology Uppsala-Sweden ${ }^{2}$

Purpose: Incomplete hippocampal inversion (IHI) is more common in patients with epilepsy than in subjects without epilepsy but is probably not an etiological factor. IHI frequency varies in different types of epilepsy. Our purpose was to evaluate the hippocampi of patients having focal epilepsy with unknown etiology and without focal abnormalities on MRI (EPue).

Methods: MRIs of 58 patients with EPue and 147 neurologically healthy controls were evaluated. Hippocampal volumetry could be performed in 54 of the patients. 47 controls, preferably those having IHI, were chosen for volumetry. The findings were compared with seizure semiology and EEG findings.

Results: $30 / 58$ patients (52\%) had IHI (18 left, 12 bilateral). 28/147 controls $(19 \%)$ had IHI $(20$ left, 8 bilateral) $(\mathrm{p}<0.001)$. In subjects studied with volumetry, $27 / 54$ patients $(50 \%)$ and $23 / 47$ selected controls (49\%) had IHI. In patients, IHI was found on the left in 15 and bilaterally in 12 . In controls, the numbers were 16 and 5 , respectively. The left hippocampus was smaller in 48 patients and in 46 controls. Asymmetry index (AI) was $>0.10$ in 16 patients $(30 \%)$ and in 3 controls $(6.5 \%)(\mathrm{p}<0.01)$. Among 10 patients having IHI and AI $>0.10$, six had temporal lobe semiology. One of them had bilateral IHI, 5 had IHI on the left. EEG foci were ipsilateral to IHI in 3 , contralateral in 2 .

Conclusions: IHI was significantly more common in EPue patients than in controls. Hippocampal volume asymmetry was more prominent in the patients. Temporal semiology and EEG focus were not obviously related to $\mathrm{IHI}$.

\section{1-0.17}

Category: Diagnostic Brain

Presentation Type : Oral Presentation

Keywords : Diffusion MRI, Glioblastoma multiforme, Treatment response

Treatment effect by advanced MR imaging in adult glioblastoma patients including analysis of regional diffusion variations

Anna Rydelius ${ }^{1}$, Björn Lampinen ${ }^{2}$, Jimmy Lätt ${ }^{3}$, Johan Bengzon ${ }^{4}$, Assa Lilja ${ }^{5}$, Danielle van Westen ${ }^{6}$, Sara Kinhult ${ }^{7}$, Silke Engelholm ${ }^{7}$, Pia C Sundgren ${ }^{6}$

Department of Clinical Sciences/ Radiology Lund University Neurology, Medi Lund-Sweden ${ }^{1}$ Department of Clinical Sciences/ Radiology Lund University Medical Radiation Physics Lund-Sweden ${ }^{2}$ Department of Clinical Sciences Lund University Radiology Lund-Sweden ${ }^{3}$ Department of Clinical Sciences Neurosurgery Lund-Sweden ${ }^{4}$ Department of Clinical Sciences Neuropsychology Lund-Sweden ${ }^{5}$ Department of Clinical Sciences Radiology Lund-Sweden ${ }^{6}$ Department of Clinical Sciences Oncology Lund-Sweden ${ }^{7}$

Background: Early detection of response to treatment is important for the management of glioblastoma patients
Purpose: To evaluate the use of MR diffusion parametric for early detection of treatment response in high grade gliomas

Material: Prospective longitudinal study of 30 high grade glioma patients (aged 34-71, median age 58 years) treated with conventional radiotherapy of $60 \mathrm{~Gy}$ with concomitant Temozolomide (TZM), and depending on clinically status received adjuvant TZM. All patients has undergone conventional brain imaging including diffusion, perfusion and MR spectroscopy at a 3T MR scanner at baseline, 1, 3, 6 weeks, 3,6,12 months, cognitive testing at baseline, 3, 6 and 12 months, and routine clinical follow-up. Regional mean diffusivity (MD) changes are being voxel-wise analysed by parametric response maps (PRM).

Results: At 8 months $12 / 30$ patients had clinically stable disease (SD), 16/30 patients had progressive disease (PD), 2 had died. A pilot analysis of 5 patients was performed aiming to see if MD-PRM pattern may predict early therapy response at three weeks after radiotherapy start. 2/5 patients showed clear MD-PRM increase; significant change being defined $>0.55 \mu \mathrm{m} 2 / \mathrm{ms}$, the same patients having clinical and radiological SD 8 months after operation whereas $3 / 5$ patients had low or no increase $<0.55 \mu \mathrm{m} 2 / \mathrm{ms}$ or in one case decrease in MD-PRM three weeks into therapy, these patients had PD after 8 months of consequent therapy. Further analysis of the remaining patients are ongoing.

Conclusion: Our pilot data support the use of MD-PRM for early detection of treatment response already at 3 weeks into.

\section{1-O.18}

Category: Diagnostic Brain

Presentation Type : Oral Presentation

Keywords : superficial siderosis, dural defects

Infratentorial superficial siderosis: clinical and radiological spectrum, causes, diagnostic criteria and rational investigation pathway based on an observational case series of 64 patients

Peter Cowley $^{1}$, Duncan Wilson ${ }^{2}$, David Werring ${ }^{2}$, Fiona Chatterjee ${ }^{3}$

NHNN Neuroradiology London-United Kingdom ${ }^{1}$ NHNN Neurology London-United Kingdom ${ }^{2}$ RLH Neuroradiology London-United Kingdom ${ }^{3}$

Background: Superficial siderosis of the central nervous system describes haemosiderin deposition in the subpial layers of the brain, cranial nerves and spinal cord, which is increasingly detected by MRI. Infratentorial superficial siderosis (iSS) is classically associated with slowly progressive hearing loss, anosmia, ataxia, and myelopathy, and attributed to a slow or recurrent subarachnoid haemorrhage. There are no standardized diagnostic criteria for iSS, the causes and optimum investigation strategy are not established. We therefore set out to describe the clinical and radiological spectrum and causes and develop a rational investigation algorithm for iSS

Results: We identified 64 patients with iSS, which we classified into two groups. The first group (Type One (classical)) included 47 patients without obvious intracranial haemorrhage. Of these, $39(83 \%)$ had hearing loss, ataxia or myelopathy. The second group (Type Two (secondary)) included 17 patients with an obvious radiologicallyconfirmed spontaneous or traumatic intracranial haemorrhage. Of these, none had hearing loss, ataxia or myelopathy. Of the 47 patients with Type One (classical) iSS, $39(83 \%)$ had a potentially causal dural abnormality identified. 5 patients $(11 \%)$ had an alternative cause, while only 3 patients $(6 \%)$ had no cause identified (all 3 had inadequate spinal imaging). Digital subtraction angiography did not identify any underlying lesions.

Conclusion: Type $1 \mathrm{SS}$ is associated with a very high incidence of spinal or posterior fossa dural defects. 
1-0.19

Category: Diagnostic Brain

Presentation Type : Oral Presentation

Keywords : Aspartylglucosaminuria, Lysosomal storage disease, MRI, Diffusion tensor imaging, Tract-based spatial statistics

Diffusion tensor imaging with tract-based spatial statistics reveals widespread microstructural alterations in aspartylglucosaminuria

Anna Tokola ${ }^{1}$, Nina Brandstack ${ }^{1}$, Antti Hakkarainen ${ }^{1}$, Jaana Hiltunen ${ }^{1}$, $\overline{\text { Laura Åberg }}^{2}$, Taina Autti ${ }^{1}$

HUS Medical Imaging Center, University of Helsinki and Helsinki University Hospital Radiology Helsinki-Finland ${ }^{1}$ Department of Psychiatry, University of Helsinki and Helsinki University Hospital Psychiatry Helsinki-Finland ${ }^{2}$

Introduction: Aspartylglucosaminuria (AGU) is an inherited lysosomal storage disease causing progressive decline in mental and motor functions. Our aim was to examine microstructural changes of WM for the first time in AGU using tract-based spatial statistics (TBSS).

Methods: A DTI series (ssDW sequence, TR $10600 \mathrm{~ms}$, TE $59,5 \mathrm{~ms}, 16$ diffusion gradients, 32+1 directions, NA 2, matrix $112 \times 112$, FOV $224 \times 224, b=1000 \mathrm{~s} / \mathrm{mm} 2,1 \mathrm{~b}=0$ ) was collected from thirteen AGU patients $(5 \mathrm{M}, 8 \mathrm{~F}$, age $9-45$ years) and thirtynine age- and gender-matched healthy controls $(3: 1)$ on a 3.0 Tesla scanner (Achieva, Philips, The Netherlands). Voxelwise statistical analysis of the DTI data was carried out using TBSS (Smith et al 2006). Two patients and their controls were excluded due to motion artefact.

Results: In patients, FA was decreased, and MD and RD increased in most of the WM tracts when compared to controls, excluding the posterior limbs of capsula interna and the splenium of $\mathrm{CC}$ for FA, the anterior and posterior limbs of capsula interna and inferior longitudinal fasciculus for $\mathrm{MD}$, and the splenium of $\mathrm{CC}$ and middle cerebellar peduncles for $\mathrm{RD}$. $\mathrm{AD}$ was increased in patients in the thalami, capsula externas, $\mathrm{CC}$, fornix and forceps major, and decreased in posterior limbs of capsula interna and cerebral peduncles.

Conclusions: TBSS revealed widespread microstructural alterations and increased overall diffusion in AGU. The findings indicate extensive axonal disintegration and dys- or demyelination, but some areas myelinating early in normal development seem to be preserved

\section{1-0.20}

Category: Diagnostic Brain

Presentation Type : Oral Presentation

Keywords : Brain atrophy, diabetes mellitus type 2

\section{A Voxel-based morphometry study in diabetes miellitus type II com- pared to healthy controls. Preliminary results of the PRECISED study.}

Alex Rovira ${ }^{1}$, Pilar Delgado ${ }^{2}$, Rafael Simó ${ }^{3}$, Jordi Bañeras ${ }^{4}$, David Garcia-Dorado ${ }^{4}$, Deborah Pareto ${ }^{5}$

Vall d'Hebron University Hospital Radiology Barcelona-Spain ${ }^{1}$ University Hospital Vall d'Hebron Neurovascular Barcelona-Spain ${ }^{2}$ Vall d'Hebron University Hospital Endocrine and Metabolism Barcelona-Spain ${ }^{3}$ University Hospital Vall d'Hebron Cardiovascular Barelona-Spain ${ }^{4}$ University Hospital Vall d'Hebron Radiology Barcelona-Spain ${ }^{5}$
Aim: The goal of this study was to investigate brain volume differences between diabetes mellitus type 2 (DM2) patients and healthy controls. Material and Methods: 58 DM2 were included in the study $(68 \%$ females, mean age $(\mathrm{SD})=66.91$ (5.91) years). A group of 15 controls was also included $(71 \%$ females, mean age $(S D)=62.71$ ( 7.24$)$ years). Brain volume differences were estimated at a regional level, by using a voxel-based morphometry (VBM) analysis (Statistical Parametric Mapping version 8). Images were segmented and normalized, following the established VBM pipeline. Then, for the statistical comparison differences were considered significant at a $p<0.05$ Family Wise Error (FWE)-corrected level and a extend threshold of 10 voxels. Age and total intracranial volumes were added as covariates in the statistical comparison.

Results: DM2 patients showed a significant grey matter loss in the right inferior temporal cortex (MNI coordinates $=(44,-69,5) ; \mathrm{p}=0.004$ and $\mathrm{k}=61$ voxels $)$ and the right supramarginal gyrus $((57,-28,27) ; \mathrm{p}=0.026$ and $\mathrm{k}=57$ voxels). No significant differences were found between groups for the segmented white matter.

Conclusions: DM2 patients showed a significant grey matter loss, compared to healthy controls, which affects the right temporal and the parietal lobe. Brain white matter does not seem to show a pattern that differed from healthy controls.

1-0.21

Category: Diagnostic Brain

Presentation Type : Oral Presentation

Keywords : Fabry's Disease; Pulvinar Sign; quantitative MRI; QSM

Redefining the Pulvinar Sign in Fabry's Disease: a Quantitative MR Study

Sirio Cocozza ${ }^{1}$, Antonio Pisani ${ }^{2}$, Gaia Olivo ${ }^{1}$, Eleonora Riccio ${ }^{2}$, Pasquale Borrelli ${ }^{3}$, Silvia Migliaccio ${ }^{2}$, Amedeo Cervo ${ }^{1}$, Arturo Brunetti ${ }^{1}$, Giuseppe Palma ${ }^{4}$, Enrico Tedeschi ${ }^{1}$

University of Naples "Federico II" Department of Advanced Biomedical Sciences Naples-Italy ${ }^{1}$ University of Naples "Federico II" Department of Public Health Naples-Italy ${ }^{2}$ IRCCS SDN IRCCS SDN Naples-Italy ${ }^{3}$ National Research Council Institute of Biostructure and Bioimaging Naples-Italy ${ }^{4}$

Purpose: The pulvinar sign, the hyperintensity on T1-weighted images of the posterior thalamus supposedly due to dystrophic calcifications, has been suggested to be a feature of Fabry's disease (FD), being reported in around $20 \%$ of male patients. Aim of this study was to perform the first quantitative MRI (qMRI) study of the pulvinar in FD, along with a retrospective evaluation of conventional MR scans of a relatively large FD population, to understand the real incidence and pathophysiology of this sign.

Material and Methods: We evaluated T1-weighted images of 56 FD patients. In a subgroup of 14 patients, both single- and double-echo 3D spoiled GRE sequences were available for the calculation of R1, R2* and QSM maps. qMRI values were assessed by hand-drawn ROI on the slice where pulvinar was best represented and were matched with those obtained in 20 age- and sex-matched healthy controls.

Results: In the whole group, only 1 male subject $(1.7 \%, 5.2 \%$ of the male population) showed a T1-weighted pulvinar hyperintensity. When evaluating qMRI maps, no significant differences were observed between healthy controls and FD patients for any of the tested map

Conclusion: In our cohort, the pulvinar sign shows a significantly lower incidence than previously reported. Furthermore, the qMRI analysis showed that no calcifications are present in the pulvinar of FD patients, allowing us to state that pulvinar sign should not be considered as characteristic of FD. 
1-0.22

Category: Diagnostic Brain

Presentation Type : Oral Presentation

Keywords :

Improved Detection Rate of Hyperacute Infarction Using Brain CT with Color Look-Up-Table

$\underline{\text { Kuei-Hong Kuo }}{ }^{\text {I, }}$, Yi-Ju Pan ${ }^{2}$, Yen-Jun Lai ${ }^{1}$

Far Eastern Memorial Hospital, Medical Imaging Division, New Taipei city-Taiwan ${ }^{1}$ Far Eastern Memorial Hospital, Department of Psychiatry, New Taipei city-Taiwan ${ }^{2}$

Purpose: Identification of hyperacute infarctions is essential for stroke management. To obtain high contrast between normal and infarcted tissues, radiologists need to narrow CT windows to search for the lesions. Compared to gray images which depend solely on differences in luminance, color images are capable of illustrating various Housefield Units (HU) in a combination of three factors, hue, luminance, and saturation. Given that color images permit a wider HU window with several partitions and each partition can have higher contrasts, we aimed to test whether brain CT with color Look-Up- Table (cLUT) improves detection rates of hyperacute infarctions.

Material and Method: We designed a non-linear cLUT for hyperacute infarctions with specific colors assigned according to HUs of CSF, white matter, gray matter, venous sinus and bone, respectively. Gray and colorcoded CT images of 14 cases with hyperacute infarctions along with those of 16 cases with other brain diseases were examined by 2 radiologists, and compared by image types.

Results: There were 14 cases with 27 infarcted areas (ACA: 1; MCA: 16; Basal ganglia: 8; and PCA: 2) and 10 cases with other diagnoses (aging: 2; old lacune : 4; ICH: 4). The two radiologists (A, B) correctly identified 23 and 17 out of the 27 infarcted areas respectively using gray images. With assistance of color-coded CT images, radiologist A identified 26 and B identified 24 infarcted areas with assistance of color-coded CT images. There were in total 2 pseudopositive cases.

Conclusion: Color-coded brain CT may help improve detection rates of hyperacute

\section{1-0.23}

Category: Diagnostic Brain

Presentation Type : Oral Presentation

\section{Mystery of polymicrogyria}

$\underline{B i l j a n a ~ V u k o m a n c i c}{ }^{1}$, Aleksandra Terzic-Beljakovic ${ }^{1}$, Marija Pesovic${\text { Grbovic }{ }^{1} \text {, Gordana }}$ Milenkovic ${ }^{2}$

Special Hospital for cerebrovascular diseases Department of radiology Belgrade-Serbia ${ }^{1}$ Euromedik General Hospital Department of radiology Belgrade-Serbia ${ }^{2}$

Purpose: Polymicrogyria (PMG) is a disorder of neuronal migration, resulting in a cortical malformation characterized by an excessive number of small, partly fused, convolutions gyri on the surface of the brain. PMG can be associated with other cortical malformations or can be seen in many syndromes.

The role of magnetic resonance imaging (MRI) as the non-invasive medical test is to improve anatomical details of the brain which aid in correct diagnosis.

Method: We present six patients, 4 female, and 2 male, aged between 19 and 65 with clinical symptoms of epilepsy, developmental delay, cognitive impairment and focal neurologic deficits. The examinations included 16 MDCT GE in one patient. MRI (1.5T, GE) was performed in all patients according to standard clinical protocols, and in one patient MRS.

Results: In all six patients neuroimaging shows irregular cortical thickening with irregularity at the gray-white matter junction, in 5 patients unilateral, and in one patient bilateral cerebral polymicrogyria. Schizencephaly, cortical dysplasia, porencephalic cyst, agenesis of the corpus callosum were some of the findings.

Conclusion: The pathogenesis of PMG is unknown, neuronal migration disorder, injury, infections, fetal ischemia, exposure to toxins or chromosomal mutations. MRI is essential to demonstrate the morphology, distribution, and extent of the disorders of cortical formation and associated anomalies.

The oldest patient, who has the most anomalies including PMG, didn't have any clinical problems until he had a brain stroke, whereas the other patients with more discrete imaging have worse neurological symptoms irrespective of where the PMG is located.

\section{1-0.24}

Category: Diagnostic Brain

Presentation Type : Oral Presentation

Keywords : Postcontrast STIR, Intracranial disease processes, Magnetic resonance imaging

\section{Postcontrast STIR Imaging of Intracranial Disease Processes - a Pilot Study}

Antonija Ruzic Barsic ${ }^{1}$, Slavica Kovacic ${ }^{1}$, Damir Miletic ${ }^{1}$, Zoran Rumboldt $^{2}$

Clinical Hospital Center Rijeka Radiology Rijeka-Croatia ${ }^{1}$ Medical University of South Carolina Department of Radiology and Radiological Sciences Charleston South Carolina Radiology Charleston-United States $^{2}$

Objectives: To asses postcontrast Short Tau Inversion Recovery (STIR) MR images for potential new information in various intracranial disease processes.

Background: STIR suppresses signal from tissues with short T1 values. Thus accumulation of gadolinium-based contrast agent leads to a relative signal loss compared to precontrast STIR images. To the best of our knolwedge, intracranial postcontrast STIR imaging has not yet been evaluated.

Methods: Sixty-three patients underwent brain MRI on a 1.5 Tesla scanner: 19 with multiple sclerosis (MS), 16 with metastases, 14 with meningiomas, 12 with gliomas and 2 with abscesses. Axial T1weighted and STIR images were obtained before and after intravenous administration of contrast agent. The enhancing lesions were analyzed for the presence and characteristics of relative signal change on postcontrast STIR images.

Results: Enhancing MS lesions revealed a surprising range of appearances on postcontrast STIR images, from a definite signal loss to a slight increase in signal intensity, which were in some cases present within the same lesion. Only some of the homogenously enhancing metastases demonstrated signal loss. Among the homogenously enhancing meningiomas two different patterns of signal loss were observed.

Conclusion: Our pilot study demonstrates that postcontrast STIR imaging offers additional information, not obtained with other sequences, suggesting various lesion characteristics, primarily in patients with MS, metastases and menigiomas. Further investigation is necessary to evaluate for the underlying cause and possible clinical utility of this novel information. 
1-0.25

Category: Diagnostic Brain

Presentation Type : Oral Presentation

Evaluation of changes in the selected white matter tracts in $\mathrm{HCV}$ infected patients using diffusion tensor imaging before and after new interferon-free therapy.

Joanna Bladowska ${ }^{1}$, Katarzyna Fleischer-Stepniewska ${ }^{2}$, Anna Zimny ${ }^{1}$, Malgorzata Inglot $^{2}$, Weronika Rymer ${ }^{2}$, Brygida Knysz ${ }^{2}$, Marta Waliszewska-Prosol ${ }^{3}$, Maria Ejma ${ }^{3}$, Marek Sasiadek ${ }^{1}$

Wroclaw Medical University Department of General Radiology, Interventional Radiology and Neuroradiology Wroclaw-Poland ${ }^{1}$ Wroclaw Medical University Department of Infectious Diseases, Liver Diseases and Acquired Immune Deficiencies Wroclaw-Poland ${ }^{2}$ Wroclaw Medical University Departemnt of Neurology Wroclaw-Poland ${ }^{3}$

Purpose: The aim of the study was to evaluate the selected white matter tracts using diffusion tensor imaging (DTI) in HCV-positive patients before and at 24 week after interferon-free therapy.

Material and Methods: Ten HCV-positive patients (5F, 5M; mean age 50.1 ) treated with ombitasvir/paritaprevir/ $\mathrm{r}$ and dasabuvir \pm ribavirin were enrolled in the study. DTI examinations were performed with $1.5 \mathrm{~T}$ MR scanner. Fractional anisotropy (FA) and average diffusion coefficient (ADC) values were obtained with a small ROI method in middle cerebellar peduncles (MCP), inferior longitudinal fasciculi (ILF), inferior frontooccipital fasciculi (IFOF), genu (GCC) and splenium (SCC) of the corpus callosum, posterior limbs of the internal capsules (PLIC), superior longitudinal fasciculi (SLF) and posterior cingula (CG). Sustained viral response was analyzed.

Results: Significant increase of FA values $(p<0.05)$ after therapy compared to values obtained before treatment was found in almost all white matter tracts apart from the right SLF and right CG. Significant decrease of $\mathrm{ADC}$ values $(\mathrm{p}<0.05)$ after treatment was observed in the right IFOF, SCC, left PLIC, left SLF, left CG. In a patient with the most advanced liver disease increase of FA value was less $(p=0.0004)$ and decrease of $A D C$ value was more $(p=0.0002)$ pronounced compared with the group. All patients achieved a sustained viral response.

Conclusion: Efficient interferon-free therapy leads to white matter tracts recovery. This is the first report on impact of interferon-free therapy on microstructural cerebral changes in chronic hepatitis $\mathrm{C}$ patients.

\section{1-0.26}

Category: Diagnostic Brain

Presentation Type : Oral Presentation

Keywords : glioblastoma, 3D-tumor-volumetry, computer-assisted, diagnostic, semi-automated, assessment

\section{Diagnostic Value of Longitudinal Computer-assisted 3D-volumetry in Follow-up MRI of Glioblastoma}

Georgina Alber ${ }^{1}$, Stefanie Bette ${ }^{1}$, Tobias Boeckh-Behrens ${ }^{1}$, Esther Alberts ${ }^{1}$, Jens Gempt ${ }^{2}$, Florian Ringel ${ }^{2}$, Claus Zimmer ${ }^{1}$, Jan Kirschke ${ }^{1}$, Thomas Huber ${ }^{1}$

Klinikum rechts der Isar, Technical University of Munich Department of Neuroradiology Munich-Germany ${ }^{1}$ Klinikum rechts der Isar, Technical University of Munich Department of Neurosurgery Munich-Germany ${ }^{2}$

Background: In glioblastoma (GB) follow-up MRI plays a key role for disease monitoring. Semi-automated 3D-volumetry is increasingly seen as an additional tool in image interpretation. Aim of this study was to investigate if semi-automated volumetry has an additional diagnostic benefit in the clinical routine.
Methods: In this retrospective study 330 longitudinal segmentations of 30 GB patients $(22 \mathrm{~m} ; 60+/-15 \mathrm{y})$ were assessed, using semi-automated volumetry. Contrast-enhancing tumor volume (CEL) was measured in a 3D-MPRage sequence (1 $\mathrm{mm}$, isotropic), perifocal FLAIR-changes (NEL) were assessed in a 3D-FLAIR sequence $\left(0,9 \times 0,9 \times 1,2 \mathrm{~mm}^{3}\right)$. The radiologic report (RR) was categorized (initial diagnosis, post-op, regression, uncertain progression, progression) and correlated with the volume change (d) and the direction of change (v).

Results: RR showed a higher correlation to $d(C E V)(r=0.692, p<0.001)$ than $d(N E L)(r=0.448, p<0.001)$. Considering significant volumetric changes with respect to intra-rater-variability, only $\mathrm{v}(\mathrm{CEL})$ was significantly associated with $\mathrm{RR}(\mathrm{r}=0.452, \mathrm{p}<0.001)$. For cases with ,uncertain progression" $(\mathrm{n}=17)$ no significant correlation could be shown between volumetry and further follow-up assessments ( $p>0.05)$.

Discussion: Precise volumetry of the contrast-enhancing tumor part using semi-automated state of the art software can substantiate the radiologic assessement, whereas associated FLAIR-changes are more variable. In case of uncertain disease progression no additional diagnostic benefit could be shown. However, focal changes might be more important than total tumor volume and could be easily visualised using $3 \mathrm{D}$-volumetry.

\section{1-0.27}

Category: Diagnostic Brain

Presentation Type : Oral Presentation

Keywords : Anorexia nervosa, MRI, MP2RAGE, cerebral atrophy

\section{Anorexia nervosa and MP2RAGE}

José Boto ${ }^{1}$, Georgios Gkinis ${ }^{2}$, Alexis Roche ${ }^{3}$, Bénédicte Maréchal ${ }^{3}$, Nadia Ortiz $^{2}$, Karl-Olof Lövblad ${ }^{1}$, François Lazeyras ${ }^{4}$, Maria Isabel Vargas ${ }^{1}$

Geneva University Hospital Department of Neuroradiology GenevaSwitzerland ${ }^{1}$ Geneva University Hospital Department of Psychiatry Geneva-Switzerland ${ }^{2}$ Advanced Clinical Imaging Technology (HC CEMEA SUI DI BM PI), Siemens Healthineers AG. LTS5, École Polytechnique Fédérale de Lausanne. Department of Radiology, Vaud University Hospital (CHUV). Lausanne-Switzerland ${ }^{3}$ Geneva University Hospital Department of Radiology Geneva-Switzerland ${ }^{4}$

Introduction: Anorexia nervosa (AN) is an eating disorder characterized by excessive weight loss and inability of the patient to understand the gravity of the situation. Cerebral atrophy has been described in these patients. The purpose of our study is to perform automated cerebral segmentation based on the MP2RAGE (Magnetization-Prepared 2 Rapid Acquisition Gradient Echo) MRI sequence in order to estimate total brain volume loss as well as volume loss of key brain structures.

Material and Methods: 13 patients (12 females, 1 male, age 16-48 years, BMI 10-20.7) with clinical suspicion of AN were analyzed with a $3 T$ Magnetom Prisma (Siemens Healthcare, Germany). The imaging protocol included conventional sequences (T2, T1, 3D FLAIR) and a MP2RAGE sequence $($ TI1/TI2/TR $=700 / 2500 / 5000 \mathrm{~ms})$. Automated segmentation of different brain structures was performed based on the MP2RAGE images with the MorphoBox prototype software.

Results: There was a statistically significant loss in total brain $(\mathrm{p}<0.001)$, gray-matter $(p=0.002)$ and white-matter $(p<0.001)$ volume as well as in the volume of the insula $(\mathrm{p}=0.025)$ and cerebellum $(\mathrm{p}=0.003)$ in the study group when compared to the general population. This group also showed a significantly higher $(\mathrm{p}<0.001)$ volume of cerebrospinal fluid. Additionally, a significant correlation was found between BMI and cerebellar volume in the study group $(r=0.675, \mathrm{p}=0.011)$.

Conclusion: Cerebral segmentation based on MP2RAGE seems to be a valuable tool to measure cerebral atrophy and atrophy of key brain structures in anorexic patients. In addition, this method allows improved follow-up of these patients. 
1-0.28

Category: Diagnostic Brain

Presentation Type : Oral Presentation

Keywords : sensorimotor area, intraoperative MRI, resting-state functional MRI, tumour

Intraoperative resting-state fMRI for lesions related to the sensorimotor area: Technical implementation and preliminary results

Ayisha Al Busaidi ${ }^{1}$, Constantin Roder ${ }^{2}$, Edyta Charyasz $^{3}$, Uwe Klose ${ }^{3}$, Marcos Tatagiba $^{2}$, Sotirios Bisdas ${ }^{1}$

University College London Neuroradiology London-United Kingdom ${ }^{1}$ Eberhard Karls University Neurosurgery Tübingen-Germany ${ }^{2}$ Eberhard Karls University Neuroradiology Tübingen-Germany ${ }^{3}$

Objective: To implement resting-state functional MRI (RS-fMRI) in an intraoperative MR setting, to post-process the data related to the sensorimotor network, to correlate them with the clinical examination and to compare with the intraoperative monitoring (IOM) performance.

Methods: Fifteen patients underwent iMRI-guided resection of lesions related to the sensorimotor area. RS-fMRI was performed pre- and intraoperatively and was correlated with patients' postoperative clinical condition, as well as with IOM findings (motor and sensory evoked potentials (MEP and SEP)). Independent component analysis (ICA) was used to postprocess the RS-fMRI data focusing on the derived sensorimotor networks. Results: Intraoperative RS-fMRI showed a significant $(\mathrm{p}<0.01)$ decrease of z-scores in patients with new postoperative neurological deficits, compared to those without postoperative complications. Significantly correlated was also the RS-fMRI score with the direct postoperative neurological status $(\mathrm{p}<0.01)$. ROC curve analysis demonstrated moderate prognostic value of intraoperative RS-fMRI z-score (cut-off value: $\leq 10.5$, sensitivity: $82.4 \%$, specificity: $80 \%, \mathrm{P}<0.0001$ ) for the postoperative paresis score. Concerning the prognostic value of IOM for postoperative paresis, the positive and negative predictive values for MEP were $99.3 \%$ and $63.6 \%$ (20\% sensitivity, $99.9 \%$ specificity), for SEP $39.9 \%$ and $57.1 \%$ ( $40 \%$ sensitivity, $57 \%$ specificity), respectively. The RS-fMRI activation of the sensorimotor areas in the contralateral hemisphere did not demonstrate any significant difference before and after operation. Conclusion: The use of intraoperative RS-fMRI for sensorimotor function mapping is feasible demonstrating prognostic value for the postoperative neurological outcome. Intraoperative RS-fMRI may act as a surrogate for IOM in lesions related to the sensorimotor area.

\section{1-0.29}

Category: Diagnostic Brain

Presentation Type : Oral Presentation

Keywords : epilepsy, gray matter, graph theory, malformations of cortical development, focal cortical displasia, FCD, MCD

Graph theory analysis of single subject gray-matter structure in malformations of cortical development (MCDs) - a novel approach for diagnostic workup

Lajos Rudolf Kozák ${ }^{1}$, Gyula Gyebnár ${ }^{1}$, Zoltán Klimaj ${ }^{1}$, Gábor Rudas ${ }^{1}$, Dániel Fabó ${ }^{2}$, Péter Barsi ${ }^{1}$

Semmelweis University MR Research Center Budapest-Hungary ${ }^{1}$ National Institute of Clinical Neurosciences Department of Epileptology Budapest-Hungary ${ }^{2}$

Purpose: Tijms et. al recently showed significant differences in MRIderived gray matter (GM) graph metrics in Alzheimer disease. As
MCDs are important causes of drug-resistant epilepsy, we aimed to investigate whether calculating similar metrics may help in diagnostic and therapeutic decision making in epilepsy patients with MCDs.

Materials and Methods: High resolution 3D T1W volumes of 31 controls and 20 patients with MCDs acquired on a 3T Philips Achieva scanner were analyzed. MCD types included focal cortical dysplasia (14), polymicrogyria (7), schizencephaly (2), and subependymal heterotopia. We used our own scripts and the SPM12, Single Subject Gray Matter Networks and Brain Connectivity toolboxes for GM graph analyses. We calculated global and a spatially transformed voxel-wise local graph metrics, assessed voxel-wise withincontrol stability using the leave-one-out approach and single patient vs. control group differences using Z-scores.

Results: Significant global GM graph differences were found in patients (higher graph density and global efficiency, lower characteristic path length and betweenness centrality, $\mathrm{p}<0.005$ for all comparisons). For the graph density metric ROC analysis yielded $84 \%$ sensitivity and $90 \%$ specificity. Moreover, the voxel-wise significance distributions of local graph metrics matched the spatial locations of MCDs in most cases.

Discussion: Graph theoretical analysis of GM similarities may be valuable in the diagnostics of MCDs. Nevertheless, further investigation is required to establish the clinical potential of this approach as the causes and effects of changes in GM graph metrics are not yet fully understood. Grant support: KTIA NAP 13-1-2013-0001; Bolyai Research Fellowship (KLR)

\section{2- Interventional}

2-0.1

Category: Interventional

Presentation Type : Oral Presentation

Keywords : transbrachial, coil embolization, cerebral aneurysms

Utility of Transbrachial Coil Embolization of Cerebral Aneurysms in the Anterior Cerebral Circulation

Takahisa Mori ${ }^{1}$, Yuhei Tanno ${ }^{1}$, Shigen Kasakura ${ }^{1}$, Kazuhiro Yoshioka ${ }^{1}$, Noriyoshi Nakai ${ }^{1}$

Shonan Kamakura General Hospital Stroke Center Stroke Treatment Kamakura-Japan ${ }^{1}$

Purpose: The aim of our study was to investigate what benefits of transbrachial coil embolization (TCE) are.

Methods: Included in our analysis were patients who underwent elective TCE in the anterior cerebral circulation (ACC) between 2014 and 2015. Evaluated were success rate of navigation of a guiding catheter and coiling procedures.

Results: During the period, 31 patients underwent elective TCE in the ACC. The 6Fr MSK-guide sheath (Medikit, Tokyo, Japan) specifically designed for transbrachial carotid cannulation was used in all patients. In 8 patients, the MSK-guide $(12 \times 90$ or $15 \times 90)$ with 0.088 inch ID was directly introduced into the target internal carotid artery (ICA), in 23 patients the $6 \mathrm{Fr}$ guiding catheter with 0.071 inch ID was coaxially introduced into the target ICA. Success rate of positioning and coiling procedures was $100 \%$, although some patients had a bovine type of the arch or the type $3 \mathrm{arch}$. Among the 31 patients, 25 patients were treated with balloon- or stent-assist techniques. Peripheral artery diseases or abdominal aortic aneurysm didn't affect TCE.

Conclusion: Procedural success of positioning was achieved in all patients. The aortic arch type, peripheral artery conditions or abdominal artery aneurysm didn't affect TCE. 
2-0.2

Category: Interventional

Presentation Type : Oral Presentation

Keywords : Intraosseous Hemangioma, Cementoplasty, Alcohol Embolization, Percutaneous, Vertebral

Long-Term Outcome of Percutaneous Alcohol Embolization Combined with Percutaneous Vertebroplasty in Aggressive Vertebral Hemangiomas with Epidural Extension

$\underline{\text { Kévin Premat }}^{1}$, Frédéric Clarençon ${ }^{1}$, Évelyne Cormier ${ }^{1}$, Jugurtha Mahtout $^{2}$, Raphaël Bonaccorsi ${ }^{3}$, Vincent Degos ${ }^{2}$, Jacques Chiras ${ }^{1}$

Pitié-Salpêtrière Hospital Interventional Neuroradiology Paris-France ${ }^{1}$ Pitié-Salpêtrière Hospital Anaesthesiology 75013-France ${ }^{2}$ PitiéSalpêtrière Hospital Orthopaedic Surgery 75013-France ${ }^{3}$

Objectives: The purpose of this study was to evaluate, on a long-term basis, the safety and effectiveness of Percutaneous Alcohol Embolization (PAE) combined with Percutaneous Vertebroplasty (PVP) as a sole treatment for aggressive vertebral hemangiomas (AVHs) with epidural extension.

Methods: From 1996 to 2015, 26 consecutive patients (15 women (58\%) and 11 men; mean age $=51.8$ y (range: $19-75$ years) underwent PAE combined with PVP (performed at Day 15) for the treatment of 27 AVHs with epidural extension. Clinical outcome was evaluated with a mean delay of $88.3 \pm 53.3$ months (range: $22-217$ months). The primary endpoint was pain relief evaluated by a Visual Analogic Scale (VAS). Results: Pre-procedure mean VAS score was $7.23 \pm 1.3$ and significantly improved at last follow up $(\mathrm{m}=3.11 \pm 1.9$; $\mathrm{P}$-value $<0.001)$. Ten patients $(38.5 \%)$ remained asymptomatic. $88 \%$ of the patients with neurosensory disorders had complete regression of these symptoms. Two of the three patients with motor deficit did not have any improvement. No major complication was recorded.

Conclusions: Exclusive treatment of AVH with epidural extension by combination of PAE and PVP is a minimally invasive safe and effective strategy.

2-0.3

Category: Interventional

Presentation Type : Oral Presentation

Keywords : aneurysm, coiling, interventional radiology, thromboembolism, antiplatelet drug resistance

Safety and efficacy of antiplatelet response assay and drug adjustment in coil embolization: A propensity score analysis

Min Soo Kim ${ }^{1}$, Kyung Il $\mathrm{Jo}^{2}$, Je Young Yeon ${ }^{2}$, Jong Soo Kim ${ }^{2}$, Keon Ha $\mathrm{Kim}^{3}$, Pyoung Jeon ${ }^{3}$, Seung Chyul Hong ${ }^{2}$

Ulsan University Hospital, University of Ulsan College of Medicine Neurosurgery Ulsan-Korea, South ${ }^{1}$ Samsung Medical Center, Sungkyunkwan University School of Medicine Neurosurgery SeoulKorea, South ${ }^{2}$ Samsung Medical Center, Sungkyunkwan University School of Medicine Radiology Seoul-Korea, South ${ }^{3}$

Background and Purpose: The purpose of this study was to explore the safety and efficacy of antiplatelet response assay and drug adjustment to prevent delayed thromboembolic events after stent-assisted coil embolization.

Materials and Methods: A total of 370 patients were enrolled in this study between December 2005 and July 2014. Of these, 124 patients were placed into the drug resistance test (DRT) group with drug adjustment according to response to an antiplatelet agent, and 246 patients comprised the control group with a standard antiplatelet regimen. The response to the antiplatelet agent was evaluated with the VerifyNow Rapid Platelet Function Assay. Propensity score matching analysis was performed with one-to-multiple matching.

Results: Among 370 patients, delayed thromboembolic events occurred in $28(7.6 \%)$ patients, $25(10.2 \%)$ in the control group and three $(2.4 \%)$ in the DRT group. Antiplatelet response test $(\mathrm{P}=0.012), \mathrm{DM}(\mathrm{P}=0.014)$, and HTN $(\mathrm{P}<0.001)$ were associated with delayed infarction in multivariate analysis. In propensity score matching analysis, 331 patients were matched (control group $(\mathrm{n}=229)$ vs. DRT group $(\mathrm{n}=103)$ ), and antiplatelet response (hazard ratio $0.247,95 \%$ confidence interval 0.070 $0.868, \mathrm{P}=0.029)$ was correlated with delayed infarction. Conversely, the two groups were not significantly different with regard to total $(\mathrm{P}=$ 0.368 ) or major hemorrhagic complications $(\mathrm{P}=0.108)$.

Conclusion: Antiplatelet drug adjustment, according to the results of an antiplatelet response assay, is effective in decreasing the risk of delayed infarction and is safe compared with the standard antiplatelet regimen

2-0.4

Category: Interventional

Presentation Type : Oral Presentation

Keywords : aneurysm, genetics, hemodynamics, anterior communicating artery

Inherited transmission of an anatomic conformation favoring aneurismal formation of the anterior communicating artery?

Cédric Lenoble ${ }^{1}$, Romain Bourcier ${ }^{1}$, Béatrice Guyomarch-Delasalle ${ }^{2}$, Benjamin Daumas-Duport ${ }^{1}$, Chrysanthi Papagiannaki ${ }^{3}$, Richard Redon ${ }^{2}$, Hubert Desal ${ }^{1}$

CHU Nantes HGRL Diagnostic and Interventional Neuroradiology Nantes-France ${ }^{1}$ Institut du thorax INSERM, UMR1087 Nantes-France ${ }^{2}$ CHU Rouen Diagnostic and Interventional Neuroradiology Rouen-France ${ }^{3}$

Object: The pathophysiological mechanisms responsible for the formation of intracranial aneurysms (IA) remain only partially elucidated. However, arguments lean in favor of a genetic component. The purpose of our study is to research specific anatomical variations in the arterial complex that are linked to the presence of anterior communicating artery (ACoA) aneurysms in the familial forms of IA.

Methods: This multi-center study included bifurcation IA in patients having an ACoA IA but no family history of IA (SACAA group), in patients having an ACoA IA and a family history of IA (FACAA), and in their healthy first-degree relatives (HFDR). Through MRI reconstructions, the symmetry of the A1 segments of the anterior cerebral arteries and the angle between the A1 and A2 segments were analyzed on threedimensional models for each group and compared.

Results: 24 SACAA patients, 24 FACAA patients, and 20 HFDR were included. Asymmetrical configuration of the A1 segments was more frequent in the FACAA group than in the HFDR $(p=0.002)$. The aneurysmside A1-A2 angle was lower in the FACAA group $(p=0.003)$ and SACAA group $(p=0.007)$ than in the HFDR group. On the other side from the aneurysm, there was no difference in A1-A2 angles between groups.

Conclusions: The anatomical shape of the anterior communicating arterial complex seems to be associated with the presence of FACAA in the same way as in SACAA. This highlights the role played by hemodynamic constraints in aneurysm formation and brings up the hypothesis of hereditary character of these anatomical shapes. 


\section{2-0.5}

Category: Interventional

Presentation Type : Oral Presentation

Keywords : acute dissection, stroke, endovascular treatment

Endovascular treatment of acute internal carotid artery dissections: technical considerations, clinical and angiographic outcome

Wiebke Kurre ${ }^{1}$, Kai Bansemir ${ }^{2}$, Marta Aguilar-Pérez ${ }^{1}$, Rosa MartinezMoreno ${ }^{1}$, Elisabeth Schmid ${ }^{3}$, Hansjörg Bäzner ${ }^{3}$, Hans Henkes ${ }^{1}$

Klinikum Stuttgart Neuroradiology Stuttgart-Germany ${ }^{1}$ Klinikum Kempten Neurosurgery Kempten-Germany ${ }^{2}$ Klinikum Stuttgart Neurology Stuttgart-Germany ${ }^{3}$

Purpose: In acute internal carotid artery dissection (a-ICAD) with concomitant intracranial large vessel occlusion or hemodynamic impairment endovascular therapy (EVT) can be considered due to limited effects of medical measures. Feasibility, safety and outcome of EVT in a-ICAD are not well described yet.

Methods: From an institutional database we selected consecutive patients treated for a-ICAD between January 2007 and July 2015. We assessed recanalization results defining $<50 \%$ residual stenosis and $\mathrm{mTICI} \geq 2 \mathrm{~b}$ successful. Adverse events and symptomatic haemorrhages were reported. mRS 0-2 at 90 days was defined as favourable clinical outcome. All available follow-up angiographies were reviewed.

Results: EVT for a-ICAD was performed in 73 patients (mean age 48 years (31-73), mean NIHSS $11(0-27))$. The majority $(60 \%)$ had tandem occlusions. ICA reconstruction was successful in $100 \%$ and intracranial thrombectomy in $85 \%$. Thrombus formation $(18 \%)$ and thromboembolism $(20 \%)$ were the most frequent adverse events but clinically relevant only in $8 \%$. Symptomatic haemorrhage occurred in $5 \%$. Clinical outcome was favourable in $64 \%$, with a lower chance after tandem occlusion $(55 \%$ vs. $79 \%$; $=0.047$ ) and $10 \%$ had died. None of the patients developed recurrent ischemic symptoms but control angiography revealed abnormal findings of the reconstructed ICA in 38\% leading to re-treatment in $17 \%$. Conclusion: EVT of a-ICAD is feasible with predominantly favourable clinical outcome. Improvement of devices and techniques is warranted to reduce the risk of thrombus formation and thromboembolism during treatment and insufficient vessel wall healing thereafter.

\section{2-0.6}

Category: Interventional

Presentation Type : Oral Presentation

Keywords : cavernous sinus; dural arteriovenous fistula; embolization

Transvenous coil embolization of cavernous sinus dural arteriovenous fistula on a revised classification

$\frac{\text { Chao-Bao Luo }}{{ }^{1} \text {, Clayton Chi-Chang Chen }}{ }^{2}$, Cherng-Gueih Shy ${ }^{3}$, HoFai Wong ${ }^{4}$

Taipei Veterans General Hospital Department of Radiology TaipeiTaiwan ${ }^{1}$ Taichung Veterans General Hospital Department of Radiology Taichung-Taiwan ${ }^{2}$ Pingtung Christian Hospital Department of Radiology Pingtung-Taiwan ${ }^{3}$ Chang Gung Memorial Hospital Department of Radiology Taoyuan-Taiwan ${ }^{4}$

Objectıve: Transvenous coil embolization is an effective method to manage cavernous sinus dural arteriovenous fistulas (CSDAVFs). However, some CSDAVFs may associate with complex angioarchitecture leading to difficult fistula access. The purpose of this study is to report our experiences of coil embolization of CSDAVFs based on a revised classification.
Methods: Over 5-year periods, there were 70 patients undergoing coil embolization of 73 CSDAVFs. There were 27 men, 43 women (mean age: 58 yrs). We retrospectively analyses the angioarchitecture of CSDAVFs and categorized these CSDAVFs into proliferative type (PT), restrictive type (RT) and late restrictive type (LRT) based on a previously revised classification. Outcomes of embolization in each type were evaluated.

Results: Pial vein reflux were depicted in $2(10 \%)$ of PT, $10(37 \%)$ in RT and $15(60 \%)$ in LRT. Para-CS components of fistula were found in 12 PT $(57 \%)$, 1 in RT (4\%) and no instance in LRT. Mean coil length for fistula occlusion of PT, RT and LRT were $432 \mathrm{~cm}, 353 \mathrm{~cm}, 85 \mathrm{~cm}$, respectively. Immediate angiographic cure of CSDAVFs were achieved in 12 (57\%) of PT, 24 $(89 \%)$ of RT and $21(100 \%)$ of LRT. No major peri-procedural complication in all CSDAVFs. The mean clinical follow-up period was 17 months.

Conclusions: Embolization outcomes may relate with various types of CSDAVFs. PT had the strong fistula flow in CSDAVFs and needed longer coils to achieve better angiographic outcome. LRT was low-flow fistula with IPS occlusion, some may be difficult to access; less coil utilization in LRT had total fistula occlusion.

2-0.7

Category: Interventional

Presentation Type : Oral Presentation

\section{Keywords :}

Development of microporous covered stent for the treatment of intracranial aneurysms (NCVC-CS1): its functional features and commencement of investor-initiated clinical trial.

Tetsu Satow ${ }^{1}$, Yasuhide Nakayama ${ }^{2}$, Eika Hamano ${ }^{1}$, Yoji Orita ${ }^{1}$, Haruko Yamamoto $^{3}$, Nobuyuki Sakai ${ }^{4}$, Hidenori Oishi ${ }^{5}$, Jun Takahashi ${ }^{1}$

National Cerebral and Cardiovascular Center Neurosurgery Suita-Japan ${ }^{1}$ National Cerebral and Cardiovascular Center Medical Engineering and Materials Suita-Japan ${ }^{2}$ National Cerebral and Cardiovascular Center Data Science Suita-Japan ${ }^{3}$ Kobe City Medical Center General Hospital Neurosurgery Kobe-Japan ${ }^{4}$ Juntendo University School of Medicine Neuroendovascular Therapy Tokyo-Japan ${ }^{5}$

We have developed a stent graft covered with a thin, microporous segmented polyurethane membrane (NCVC-CS1) for the treatment of large or giant intracranial aneurysms which are difficult to treat by conventional approach such as coiling, parent vessel occlusion, or surgical clipping. The functional testing of NCVC-CS1 and the design of investigatorinitiated clinical trial is presented.

Navigability, Aneurysm occlusion, and patency of branching vessel were assessed using the following three animal models.

(1) Easy navigation were observed by canine carotid siphon model made by inlaying anastomosed CCAs into a skeletonized acrylic tube designed on the basis of 3D rotational angiography of human ICA and 3D-printingaided manufacturing.

(2) Instant occlusions of large aneurysms, even located at the outer side of the curved arteries, were confirmed by canine sidewall, wide-necked aneurysm model.

(3) Patency of perforating arteries was affirmed by stent placement in rabbit aorta at the branching of lumbar arteries.

Upon these results, an investor-initiated, first-in human, clinical trial to evaluate the safety and efficacy of NCVC-CS1 was started in May 2016 with the support of Japanese Agency of Medical Research and Development. This trial will recruit a total of 12 patients with unruptured, wide-necked aneurysms whose diameter $>7 \mathrm{~mm}$, located in petrous/cavernous portion of ICA, intradural VA, or BA below the orifice of SCA within two years. Primary endpoints are any stroke or death related to the procedure within 180 days, and complete obliteration of target aneurysm and patency of target vessel (less than $50 \%$ stenosis) at 180 days after the procedure. 


\section{2-0.8}

Category: Interventional

Presentation Type : Oral Presentation

Keywords : Stroke, Thrombectomy, Histology, Thrombus

Preinterventional i.v.-rtpa in middle cerebral artery treated with thrombectomy: a double-edged sword?

Johannes Kaesmacher ${ }_{-}$, Justus Kleine ${ }^{1}$

Klinikum rechts der Isar, Technical University Munich Neuroradiology Munich-Germany ${ }^{1}$

Background: Recent studies suggested that I.V.-rtPA bridging facilitates successful and fast vessel recanalizations in the context of endovascular stroke treatment (EST).

Methods: Retrospective analysis of 234 consecutive patients with an acute occlusion of the middle cerebral artery, treated with upto-date endovascular techniques. Clinical outcomes and safety/ efficacy of EST, defined as rates of successful, first-pass and TICI-3-recanalizations, were evaluated for patients with and without I.V.-rtPA bridging and stratified by distal vs. proximal occlusions.

Results: Clear-cut and consistent associations between bridging-therapy and parameters of EST were present in distal MCA occlusions only, but not in proximal ones. In distal MCA occlusion, I.V.-rtPA favored successful (adjusted OR: $4.5(1.6-13.2) p=0.006)$ and one-pass vessel recanalization (aOR: 2.8 [1.0-7.6]; $\mathrm{p}=0.042$ ), but was associated with lower rates of complete reperfusion (TICI3, 0.413 [0.171-1.0], $\mathrm{p}=0.046$ ). I.V.-rtPA favored neurological improvement (NIHSS >8) in distal, (aOR: 2.8 [1.1-6.9], $\mathrm{p}=0.030$ ), but not in proximal MCA occlusions (aOR: 1.2 [0.5-2.7], $\mathrm{p}=0.812$ ).

Conclusion: I.V.-rtPA bridging facilitates successful recanalization by subsequent endovascular thrombectomy. This benefit, however, came at a cost, which was not a significantly higher rate of hemorrhage, but, rather, a lower proportion of TICI-3 vs. TICI $2 b$. Importantly, both observations held only for distal MCAocclusions and the benefits seem to outweigh the risk in terms of clinical outcomes. Occlusion-site-stratification should be considered in upcoming randomized trials evaluating the value of I.V.rtPA bridging.

\section{2-0.9}

Category: Interventional

Presentation Type : Oral Presentation

Keywords : ischemic stroke, mechanical thrombectomy, computed tomography angiography, collateral, stent retriever

Correlation of long term clinical outcome with the degree of collateral circulation in stroke patients treated with stent retrievers - Are there indications not to perform thrombectomy based on CT angiography?

Hannes Nordmeyer ${ }_{-}{ }_{-}$, Ralph Weber ${ }^{2}$, Nadine Webering ${ }^{3}$, René Chapot ${ }^{1}$ Alfried Krupp Hospital Neuroradiology Essen-Germany ${ }^{1}$ Alfried Krupp Hospital Neurology Essen-Germany ${ }^{2}$ Ev. Klinikum Niederrhein Neurology Duisburg-Germany ${ }^{3}$

Purpose: The association between baseline collateral status on CTAngiography and outcome in patients with acute anterior circulation stroke treated by mechanical Thrombectomy was investigated.

Methods: 87 consecutively treated patients from a prospective thrombectomy registry were retrospectively analyzed regarding collateral status on CTA source images, categorized by two blinded readers into good, moderate, and poor with the Tan and Miteff scores. To analyse the association between collateral score and favourable clinical outcome and death at follow-up, multivariate logistic regression was used. The association between collateral score and successful recanalization was analysed.

Results: Median NIHSS score was 15 , mean age 72.5 years. The Miteff score could be assessed in 65 of 87 patients as an occlusion of the proximal ICA or image quality prevented both readers from collateral grading in 23 patients. All 6 patients with poor collaterals on Miteff score died during follow-up.

Patients with poor collaterals on Tan score had a significantly higher mortality compared to those with moderate or good collaterals during follow-up of 5.2 months $(85.7 \%$ vs. $30.6 \%$ vs. $25.7 \%, \mathrm{p}<0.001)$. Logistic regression analysis did not show a significant association between Tan score (OR 1.681, 95\% CI 0.683-4.140) and recanalization. Higher age (OR 1.067, 95\% CI 1.013-1.124; $\mathrm{p}=0.015$ ) and lower Tan score (OR 0.421, 95\%CI 0.192-0.922; $\mathrm{p}=0.031$ ) were independent predictors of death at follow-up.

Conclusions: In anterior circulation stroke patients with poor collaterals on CTA, indication for mechanical recanalization should be made cautious because of a strong association with fatal outcome despite a high rate of recanalization.

\section{2-O.10}

Category: Interventional

Presentation Type : Oral Presentation

\section{Keywords :}

Efficacy of deconstructive method for ruptured vertebral artery dissecting aneurysms

Yoji Orita ${ }^{1}$, Tetsu Satow ${ }^{1}$, Daisuke Maruyama ${ }^{1}$, Yoshiro Ito ${ }^{1}$, Eika Hamano ${ }^{1}$, Seiichiro Eguchi ${ }^{1}$, Shunichi Tanaka ${ }^{1}$, Hirofumi Matsubara ${ }^{1}$, Hironori Fukumoto ${ }^{1}$, Hiroharu Kataoka ${ }^{1}$, Jun C Takahashi ${ }^{1}$

National Cerebral and Cardiovascular Center Neurosurgery Suita-Japan ${ }^{1}$

Background: For the treatment of ruptured vertebral artery dissecting aneurysms (RVADAs), whether deconstructive method such as internal trapping (IT) and proximal occlusion (PO) or reconstructive method using stents should be taken is a concern.

Objective: This retrospective study was aimed to evaluate the efficacy of our therapeutic strategy in which primary choice was parent artery occlusion. IT was performed in cases without branching vessels while PO executed in PICA-involved lesions or with branches arising from aneurysmal dilatation (AnD). Stents were used only when contralateral VA were occluded or hypoplastic.

Materials and Methods: A total of 45 consecutive patients who underwent NET at our institute between April 2005 and March 2016 were included. There were 34 males $(75.6 \%)$ and the mean age was 52.0 years (range, 33-80 years). WFNS grade 4 and 5 was 28 cases $(60.9 \%)$. Aneurysms were classified into 4 types according to the relationship with the origin of PICA (proximal: 3, PICA-involved: 10, distal: 25, absence of PICA: 7). Perforators or ASA arising from AnD were seen in 7 cases.

Results: Technical success was noted in all cases (IT: 27, PO: 15, Stent-assisted coiling: 3). In IT and PO, no rerupture was observed. Medullary infarction occurred only in 3 cases $(7.1 \%)$. The ratio of favorable outcome (mRS $0-2)$ was $60.9 \%$ (44.8\% in poor grade cases).

Conclusion: Prevention of rerupture and low incidence of ischemic events were achieved, along with good functional outcome by our strategy although reconstructive method might be promising. 
2-0.11

Category: Interventional

Presentation Type : Oral Presentation

Keywords : Acute Stroke, Basilar Artery Occlusion, Mechanical Thrombectomy

Endovascular treatment of acute basilar artery occlusion: RELOBA study Group experience.

Andrea Giorgianni ${ }^{1}$, Francesco Biraschi ${ }^{2}$, Dikran Mardighian ${ }^{3}$, Roberto Gasparotti ${ }^{3}$, Michele Frigerio ${ }^{3}$, Guglielmo Pero ${ }^{4}$, Luca Quilici ${ }^{4}$, Mariangela Piano $^{4}$, Mario Crispino ${ }^{5}$, Carlo Pellegrino ${ }^{1}$, Marco Pavia ${ }^{6}$, Roberto Peroni ${ }^{7}$, Marco Longoni ${ }^{8}$, Martino Cellerini ${ }^{9}$, Elvis Lafè ${ }^{10}$, Paolo Remida ${ }^{11}$, Giuseppe Faragò ${ }^{12}$, Paolo Reganati ${ }^{13}$, Sabina Strocchi ${ }^{1}$, Luca Valvassori ${ }^{4}$

Ospedale di Circolo Neuroradiology Varese-Italy ${ }^{1}$ Sapienza University of Rome Neuroradiology Rome-Italy ${ }^{2}$ Università degli Studi di Brescia Neuroradiology Brescia-Italy ${ }^{3}$ Ospedale Niguarda Ca Neuroradiology Milano-Italy ${ }^{4}$ AO Istituti Ospetalieri Radiology Cremona-Italy ${ }^{5}$ Fondazione Poliambulanza Neuroradiology Brescia-Italy ${ }^{6}$ Sant'Anna Hospital Neuroradiology Como-Italy ${ }^{7}$ A. Manzoni” Hospital/Ospedale Niguarda Ca Neurology Lecco/Milano-Italy ${ }^{8}$ Papa Giovanni XXIII Hospital Neuroradiology Bergamo-Italy ${ }^{9}$ Fondazione IRCCS Policlinico S. Matteo Neuroradiology Pavia-Italy ${ }^{10}$ Universita Neuroradiology Monza-Italy ${ }^{11}$ Neurological Institution 'C Besta' Neuroradiology MilanoItaly ${ }^{12}$ Ospedale San Carlo Borromeo Neuroradiology Milano-Italy ${ }^{13}$

Background and Objective: Acute Basilar artery (BA) occlusion is considered among the most severe medical conditions, with very high morbidity and mortality. The aim of this study is to present 5 years experience of 12 centres of the Lumbardy area -Italy-, in BA occlusion endovascular treatment (EVT) and to evaluate prognostic factors that may improve clinical outcomes and recanalization rate.

Methods: The RELOBA registry is a retrospective multicentre collection of basilar artery occlusion undergone to EVT in the period 2010-2015. 102 patients (mean age 65 years) with proven basilar artery occlusion treated with EVT were included. Clinical, procedure and neuroradiological data were collected. Recanalization (TICI score $2 \mathrm{~b}-3$ ) was assessed by local interventional neuroradiologist. Good (moderate) outcome was defined as a mRS score of 0 to $2(0-3)$ assessed at 3 months.

Results: $39 \%$ patients had good and $46 \%$ had moderate mRS clinical outcome at 3 months. Mortality was $30 \%$. TICI 2 b-3 recanalization was achieved by $61 \%$. Univariate analysis showed that age, NIHSS at onset, total time to recanalization, recanalization TICI grade were all statistically significant $(\mathrm{p}<0.05)$ in predicting clinical outcome. Multivariate logistic regression showed total time, age, NIHSS at onset as significant independent predictors of good outcome.

Conclusion: Mechanical thrombectomy is feasible and effective in patients with acute basilar artery occlusion. Better understanding of the factors that influence the prognosis could dramatically improve patient outcome. These initial results must be confirmed by further prospective studies within a randomized-controlled settings.

\section{2-0.12}

Category: Interventional

Presentation Type : Oral Presentation

\section{Keywords :}

Endovascular Treatment of Intracranial Aneurysms Using Acandis Acclino Stents: Mid- and Longterm Results

Friedhelm Brassel ${ }^{1}$, Dominik Grieb ${ }^{1}$, Dan Meila ${ }^{1}$, Martin SchlunzHendann ${ }^{1}$, Björn Greling ${ }^{1}$, Katharina Melber ${ }^{1}$
Sana Kliniken Duisburg Department of Radiology and Neuroradiology Duisburg-Germany ${ }^{1}$

Background: We report our mid- and longterm experience with the Acandis Acclino self-expanding closed-cell stents in the treatment of intracranial aneurysms.

Methods: A total number of 43 patients ( 27 female, 16 male) with intracranial aneurysms were treated with 60 Acandis Acclino stents (follow-up: 2012 2016; mean: 11 months). Dissecting aneurysms were seen in 7 patients, 36 patients presented with different types of wide-necked, fusiform and complex bifurcation aneurysms. Eleven aneurysms were treated using the kissing-Y stenting technique. We analyzed demographic data and follow-up results.

Results: The stents were successfully deployed in all cases. In three nondissecting aneurysms, sole stenting without coiling was the treatment of choice. Thirty-three non-dissecting aneurysms were treated by stentassisted coiling, showing a complete immediate aneurysmal occlusion in $30(91 \%$ RROC 1) cases. Follow-up demonstrated stable complete aneurysmal occlusion in 25 of 27 patients (93\% RROC 1). Two patients showed stable residual aneurysmal filling (RROC 3 ) without clinical significance. One residual aneurysm has been occluded on follow-up (RROC 1). In all dissecting aneurysms constructive therapy with stenting and preservation of the affected parent artery was successfully achieved. Additional subsequent coil embolization was successfully performed in 6 of the 7 dissecting aneurysms. The overall directly procedural-related complication rate was $7 \%$, including one death.

Conclusion: Endovascular treatment of various intracranial aneurysms using Acandis Acclino stents is a feasible and safe procedure with low complication rates. Even severe cases with complex bifurcation aneurysms can be treated amongst others with the kissing-Y stenting technique with good mid- and longterm

\section{2-0.13}

Category: Interventional

Presentation Type : Oral Presentation

Keywords : Ruptured blister aneurysm; flow-diverter; subarachnoid haemorrhage

Treatment of ruptured blister aneurysms with flow-diverters: a single centre experience

Chiara Gaudino ${ }^{1}$, Christian Herweh ${ }^{1}$, Mirko Pham ${ }^{1}$, Martin Bendszus ${ }^{1}$, Markus Möhlenbruch ${ }^{1}$

University Hospital Heidelberg Diagnostic and Interventional Neuroradiology Heidelberg-Germany ${ }^{1}$

Introduction: Treating ruptured blister aneurysms is difficult with coiling or surgery. Flow-diverter (FD) represent a promising treatment option. Due to double anti-aggregation therapy combined with time needed for aneurysmal occlusion, it is controversial how safe FD are in acute subarachnoid haemorrhage (SAH). We analysed safety and effectiveness of FD during the acute phase of ruptured blister aneurysms.

Materials: All patients (pts) with ruptured blister aneurysms treated with FD from $04 / 2008$ to $02 / 2016$ were retrospectively analysed. Aneurysmal occlusion and flow-diversion rate (Raymond and OKM), SAH H\&Hgrade, complications and clinical outcomes (mRS) at discharge and follow-ups were analysed.

Results: 10 pts were identified (f/m:7/3; mean age: $47 \pm 14 \mathrm{y}$; SAH H\&Hgrade: I(5), II(2), III(1), IV(2); 7/10 anterior circulation) with $2,4 \pm 0,6 \mathrm{~mm}$ mean maximal aneurysmal and $2,3 \pm 0,6 \mathrm{~mm}$ mean neck diameter and follow-up with DSA, CT and/or MRI until complete aneurysmal occlusion. $1 \mathrm{pt}$ died due to hydrocephalus during the acute phase. Pontine ischemia with complete recovery occurred in $1 \mathrm{pt}$ with FD in the basilar artery. At 6 months 9 pts had mRS $=0$. Immediately after FD implantation all aneurysms were perfused with low to moderate flow-diversion. 
Complete aneurysmal occlusion (Raymond 1; OKM D) occurred in 9 pts (7 in $\leq 3$ months, 1 in $\leq 6$ months, 1 in $\leq 10$ months). Implantation of a second FD was necessary in 2 pts, due to growing aneurysm in acute phase and failed occlusion after 24 months. No second bleeding occurred. Conclusions: Implantation of FD acutely in ruptured blister aneurysms seems safe and effective. Multicenter-studies with larger patient cohorts are necessary to confirm these results.

\section{2-0.14}

Category: Interventional

Presentation Type : Oral Presentation

\section{Keywords :}

CT Perfusion (CTP) for early detection of vascular complications after Subarachnoid Hemorrage (SH) due to aneurysm rupture.

Edoardo Puglielli ${ }^{1}$, Ruggero Lattanzi ${ }^{1}$, Federica De Filippis ${ }^{1}$, Vincenzo Di Egidio

\section{Asl Teramo Interventional Radiology Teramo-Italy ${ }^{1}$}

Purpose: Aim of this paper is to analyse the possible correlation between $\mathrm{CTP} / \mathrm{CTA}$ finding and outcome to manage $\mathrm{BP}$ in intensive care units after $\mathrm{SH}$.

Methods: 246 consecutive patients with symptomatic SH (mean age 58,7y, April 2009-March 2016), Fisher 2 (76.27\% [180/236]), 3 $(18.22 \%$ [43/236]), 4 (5.5\% [13/236]) and evidence of intracranial aneurysm that undergoing endovascular coiling, with subsequent permanent exclusion. The PCT values (CBV, CBF, MTT, TTP) and penumbra areas correlates with vascular hypoperfusion. Patients' age, sex, Hunt-Hess score at presentation and PTC values were recorded and correlated using a multiple logistic regression analysis $(\mathrm{p}<0,005)$.

Results: Reduction of CBV and increase of MTT suggestive for rise of intracranial pressure may represent an indicator of microvascular damage $(\mathrm{p}<0,005)$. Large infarct areas on CTP correlate with hemorragic suffusion (33/236) 9.74\%. (162/236) 68.64\% had a good (17-20), (40/236) $16,94 \%$ a medium (11-16), and (34/236) $14,40 \%$ a poor (0-10) rLMs. Interrater reliability was high, with an intraclass correlation coefficient of 0.87 (95\% CI, 0.77\%-0.95\%). In multivariable analysis, the rLMs (good versus poor: $\mathrm{OR}, 15.8$; $95 \%$ CI, 3.3\%-97.4\%; medium versus poor: $\mathrm{OR}$, $9.5,95 \%$ CI, $1.7 \%-50.6 \%)$. In $87.71 \%(207 / 236)$ the systolic BP greater than 240 has been correlated with poor outcomes (mRS 3-5) $\mathrm{p}<0.005$.

Conclusions: Although recent studies support the role of CTP in the early detection of vascular complications after $\mathrm{SH}$ more efforts are needed to evaluate the relationships between imaging and pressure in order to increase the early detection of ischemic damage after $\mathrm{SH}$.

\section{2-0.15}

Category: Interventional

Presentation Type : Oral Presentation

Keywords : paraganglioma, embolization, Onyx, CBT

Carotid body tumours-CBT endovascular embolisation using onyx : single centre experience

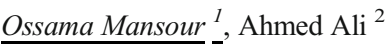

Alexandria University Neurology and Neuroradiology Alexandria-Egypt ${ }^{1}$ Alexandria University ENT Alexandria-Egypt ${ }^{2}$

Background and Purpose: Paragangliomas are tumors of neural crest origin commonly arising from the carotid body, vagal nerve, or jugular bulb. The definitive treatment for these tumors is surgical resection, often augmented with pre-operative embolization due their highly vascular nature. We present our experience examining the efficacy and safety of endovascular embolization of these rare tumors.

Methods: A review of patient's diagnosed with paragangliomas who underwent pre-operative embolisation from 2009 to 2015 was conducted. The tumor subtype, efficacy of embolization, method of embolization, and rate of complication were noted.

Results: A total of 38 patients underwent selective arterial embolization of their paraganglioma using onyx (Ethylene-vinyl Alcohol Copolymer). The tumor subtypes treated were carotid body $(\mathrm{n}=20)$, glomus vagale $(n=10)$, and glomus jugulare $(n=8)$. The average age at presentation was 44 years (range, 15-81). Twenty-two patients were female and sixteen were male. The most common artery embolized was the ascending pharyngeal branch of the external carotid artery. Post-embolization angiography revealed an average decrease in blood flow to tumor of $75 \%$. With the exception of transient facial pain documented in 1 patient, there were no known complications from embolization.

Conclusions: The endovascular embolization of paragangliomas using Onyx prior to surgical resection is a very safe and efficacious procedure that may reduce operative blood loss and associated morbidity.

\section{2-O.16}

Category: Interventional

Presentation Type : Oral Presentation

Keywords : Stroke, 3-D computational fluid dynamics, mechanical aspiration thrombectomy, balloon occlusion

\section{3-D computational fluid dynamics during mechanical aspiration} thrombectomy in acute ischemic stroke

Cecilia Haydee Corzo ${ }^{1}$, Michael Neidlin ${ }^{2}$, Martin Büsen ${ }^{2}$, Omid Nikoubashman ${ }^{1}$, Marguerite Müller ${ }^{1}$, Ulrich Steinseifer ${ }^{2}$, Martin Wiesmann ${ }^{1}$, Sarah Heringer ${ }^{1}$, Carolin Brockmann ${ }^{1}$

Universitätsklinikum Aachen Clinic for Diagnostic and Interventional Neuroradiology Aachen-Germany ${ }^{1}$ Helmholtz Institute of RWTH Aachen University \& Hospital Institute of Applied Medical Engineering Aachen-Germany ${ }^{2}$

Purpose: For endovascular stroke treatment clot extraction by stentretrievers is often combined with aspiration and balloon occlusion techniques to increase the efficacy of extraction and to reduce the risk of periinterventional thrombus dispersion. Only little is known about the quantitative effects of access catheter aspiration and balloon blockage on cerebral hemodynamics.

Methods: We aimed to investigate and quantify the influence of proximal balloon occlusion as an aspiration technique on the success of vessel recanalization using a 3-D CFD model based on real patient data generated by cerebral computer tomography angiography.

Blood flow rates and pressures values were evaluated regarding the use of balloon occlusion and proximal access catheter aspiration in two situations: (1) occlusion of the internal carotid artery (ICA) at its division into anterior and middle cerebral arteries (Carotid-T) with cross-flow along the posterior communicating artery, and (2) occlusion of the M1-Segment of the middle cerebral artery (MCA) with and without cross-flow along the anterior communicating artery.

Results and Conclusions: Proximal balloon occlusion leads to inverted blood flow in the internal carotid artery and negative pressure on the thrombus during thrombus extraction. Thus it increases the probability of successful vessel recanalization, and reduces the risk of thrombus dispersion during thrombus extraction. Proximal balloon occlusion is especially beneficial if there is an interfering cross flow from the contralateral side at the terminal internal carotid artery. The presented 3-D CFD model can be used to optimize endovascular stroke therapy. 
2-0.17

Category: Interventional

Presentation Type : Oral Presentation

Keywords : SVS, stroke

SVS : inter MRI manufacters agreement, inter and intra observers agreement, histo-MR correlation

Détraz Lili ${ }^{1}$, Bourcier Romain ${ }^{1}$, Guyomarch Béatrice ${ }^{2}$, Toquet Claire ${ }^{3}$, Serfaty Jean Michel $^{4}$, Desal Hubert ${ }^{1}$

Centre Hospitalier Universitaire Nantes Hôpital G et R Laennec Diagnostic and Interventional Neuroradiology Saint Herblain-France ${ }^{1}$ Centre Hospitalier Universitaire Nantes Hôpital G et R Laennec Centre d'investigation clinique Thorax, institut du thorax Nantes-France ${ }^{2}$ Centre Hospitalier Universitaire Nantes Hôpital G et R Laennec Histology and Pathologist Nantes-France ${ }^{3}$ Centre Hospitalier Universitaire Nantes Hôpital G et R Laennec Diagnostic cardiac and vascular imaging Nantes-France ${ }^{4}$

Background and Purpose: The susceptibility vessel sign (SVS) on gradient echo T2 weighted is known to indicate an erythrocyte-rich thrombus. The purpose of this study was to evaluate inter MRimaging agreement, inter and intra observer agreements and histo-MRimaging correlation of this sign.

Materials and Methods: 35 clots with different red blood cell (RBC)/fibrin ratios were made from fresh ovine blood and underwent a gradient echo $\mathrm{T} 2$ weighted based on the constructor parameters on machines of four different MRI manufacturers (1,5T GE, Siemens, Philips, 3T Philips). 10 radiologists have classified twice, the SVS of each clot's image in three groups: absent, doubtful, present. A three levels classification was used by the histologist to quantify the RBC proportion in each clot after MRI. Results: The inter MRI manufacturers agreement was moderate $\mathrm{k}=0,44$ (IC $[0,37-0,42])$ to good $\mathrm{k}=0,67$ (IC $[0,61-0,74])$. The inter observers agreement was good $\mathrm{k}=0,54$ (IC $[0,45-0,67])$ to excellent $\mathrm{k}=0,89$ (IC $[0,83-0,94])$. The intra observers agreement was also good to excellent $\mathrm{k}$ $=0,85$ (IC $[0,79-0,92])$. The histo-MRimaging correlation was moderate $\mathrm{k}=0,5(\mathrm{IC}[0,44-0,55]$ to good $\mathrm{k}=0,76$ (IC $[0,72-0,80]$ ).

Conclusions: Our study suggests that the SVS is a reproducible sign between observers and highly correlated to the histology composition despite a moderate to good inter MRI manufacturers agreement. Therefore, interpretation of this sign in large studies using different machines of MRI manufacturers and parameters has to be done with care.

\section{2-0.18}

Category: Interventional

Presentation Type : Oral Presentation

Keywords : Dissectiong, Fusiform, PICA, Aneurysm, Segmental Occlusion, Trapping

\section{Abstract No : 1210 \\ Non-saccular aneurysms of the posterior inferior cerebellar artery: review of a single center series.}

Joseph Gabrieli ${ }^{1}$, Frederic Clarençon ${ }^{1}$, Federico Di Maria ${ }^{2}$, Silvia Pistocchi ${ }^{2}$, Bruno Bartolini ${ }^{2}$, Jacques Chiras ${ }^{1}$, Nader Sourour ${ }^{2}$

Hôpital Pitié-Salpêtrière - UPMC Paris VI Interventional Neuroradiology Paris-France ${ }^{1}$ Hôpital Pitié-Salpêtrière Interventional Neuroradiology Paris-France $^{2}$

Object: The authors report the management and outcome of a single center series of 25 patients who received endovascular treatment for a ruptured nonsaccular PICA (posterior inferior cerebellar artery) aneurysm.
Methods: Medical records from January 2005 to April 2016 were retrieved from our institutional database.

Only ruptured isolated PICA dissecting aneurysms were included, aneurysms involving the vertebral artery and extending to the PICA were not considered.

Endovascular management, complications and angiographic and clinical follow-up were recorded.

Results: Fifty-three patients presented a ruptured isolated PICA aneurysm. Among those aneurysms $25(47 \%)$ were located in the anterior medullary segment of the PICA; 28 (53\%) beyond the anterior medullary segment. Aneurysms located in the first segment were saccular in 23 $(92 \%)$ of the cases while aneurysms located from the lateral to the cortical segment were non-saccular in $23(82 \%)$ of the cases.

Among the 25 non-saccular aneurysms one was a mycotic aneurysm, 2 were considered as possibly blister-like aneurysms and $23 \mathrm{had}$ an irregular fusiform shape.

Treatment included aneurysm sac coiling, segmental trapping, proximal occlusion or stenting and varied according to aneurysm location, type and operator preference. Partial or complete infarction of the cortical territory of the PICA occurred in $13(52 \%)$ of the cases, while infarction of the lateral medulla occurred in one case $(4 \%)$. Clinical and neuroimaging follow-up was performed in all cases.

Conclusions: Endovascular treatment of non-saccular PICA aneurysms, is effective in preventing early rebleeds. The possibility and consequences of an ischemia of the distal territory in case of parent vessel sacrifice requires individualized management.

\section{3- Head \& Neck}

\section{3-0.1}

Category: Head \& Neck

Presentation Type : Oral Presentation

Keywords : 3DTOFMRA, 2DTOFMRA, ICA, ATHEROSCLEROSIS, STENOSIS

Comparsion of contrast and non contrast 3DTOF MR angiography in detecting clinically significant stenosis of ICA(internal carotid artery)

$\underline{\text { Edin Avdagic }}{ }^{1}$, Deniz Bulja ${ }^{2}$

Clinical Center University Sarajevo neuroradilogy Sarajevo-Bosnia and Herzegovina ${ }^{1}$ Clinical Center University Sarajevo neuroradiology Sarajevo-Bosnia and Herzegovina ${ }^{2}$

Introduction: Atherosclerotic disease of internal carotid artery(ICA) is the most common cause of ischemic stroke. Because of that determining of clinically significant stenosis $\geq 70 \%$ of ICA,symptomatic patients, is the most crucial thing. Those patients are potentially candidates for surgical and endovascular treatment.

Materials and Methods: Our purpose was to evaluate contrast enhanced MRA with unenhanced 3DTOF MRA in detecting clinically significant ICA stenosis, by using CT angiography (CTA) as the reference standard. This was retrospective- prospective study. We identified 55 patients, 110 ICAs, from which, in study, we included 51 patients, with 102 ICAs. All diagnostic we have performed at UCC Sarajevo, Clinic for Radiology. We collected all data from AGFA IMPAX system. All data were analyzed statistically with SPSS version 22 . For each of diagnostic methods we used, we have performed sensitivity and specificity tests, as the accuracy tests and positive and negative predictive values. ICA stenosis was measured with five point scale according to NASCET criteria's for measurement of ICA stenosis. Results: In 102 analyzed patients the mean stenosis of ICA on CTA was $53 \%$, on CE-MRA was $56 \%$ and on 3DTOF MRA was also $56 \%$. On CTA there were 42 of 102 ICAs with stenosis $\geq 70 \%$, or $41,1 \%$, on $3 \mathrm{D}$ TOF MRA stenosis were found in 45 ICAs or $44.1 \%$, and on CE-MRA 
stenosis $\geq 70 \%$ were found in 46 ICAs or $45.1 \%$. With comparative statistics methods, Wilcox test has been showed no significant difference in detecting degree of stenosis

\section{3-O.2}

Category: Head \& Neck

Presentation Type : Oral Presentation

Keywords : apparent diffusion coefficients, Gamma Knife radiosurgery, vestibular schwannomas, Gamma Knife radiosurgery

Use of apparent diffusion coefficients to predict outcome of vestibular schwannomas following Gamma Knife radiosurgery

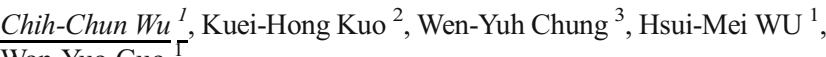
Wan-Yuo Guo ${ }^{2}$

Taipei Veterans General Hosptial Radiology Taipei-Taiwan ${ }^{1}$ Far East Memorial Hospital Radiology Taipei-Taiwan ${ }^{2}$ Taipei Veterans General Hosptial Neurological Institute Taipei-Taiwan ${ }^{3}$

Purpose: A small portion of patients with vestibular schwannomas (VS) treated by gamma knife radiosurgery (GKS) has persistent postradiosurgical tumor growth. This study is aimed to determine the MR imaging features as predictors of tumor control after GKS

Methods And Materials: We performed a retrospective study of VS patients treated with GKS between 2005 and 2013 at our institution. This study included a total of 187 patients who had a minimum of 24-months post-radiosurgical radiological assessment, and excluded those had craniotomy with tumor removal before and after GKRS. Serial MR features and apparent diffusion coefficients (ADC) values measured by placing regions of interest on whole tumor before and after GKS were obtained. Results: There were 85(45.5\%) male and $102(54.5 \%)$ female patients, with median follow-up period was 60.8 (range 24-128.9) months. At last follow-up, 150 tumors $(80.2 \%)$ showed decreased tumor volume, 20 $(10.7 \%)$ stabilized, and $17(9.1 \%)$ continued to grow following radiosurgery. The mean pre-radiosurgical ADCmax for all VS was significantly higher in those with tumor regression at last follow-up compared with those with progression or stabilization ( $2458 \mathrm{vs.} 2073 \times 10-6 \mathrm{~mm}^{2} / \mathrm{s}, \mathrm{p}=.027$ ). Conclusion: Morphological changes on MR images are common postradiosurgical phenomena but they do not predict tumor response to radiosurgery. Pre-radiosurgical ADC values and enhancing pattern are helpful to predict and evaluate post-radiosurgical outcome.

\section{3-0.3}

Category: Head \& Neck

Presentation Type : Oral Presentation

Keywords : internal carotid stenosis, T1-weighted carotid magnetic resonance image, non high-intensity signal

Plaque morphology and clinical features of symptomatic internal carotid artery stenosis with non-high intensity signal of $\mathrm{T} 1$ weighted magnetic resonance image

Megumu Takata ${ }^{1}$, Manabu Nagata ${ }^{1}$, Kazumichi Yoshida ${ }^{1}$, Takashi Takagi ${ }^{1}$, Susumu Miyamoto ${ }^{1}$

Kyoto university neurosurgery Kyoto-Japan ${ }^{1}$

Purpose: Based on T1 weighted carotid MRI, although high intensity signal is supposed to be a feature of the vulnerable plaque, some internal carotid artery stenosis (ICS) with non-high intensity signal often cause ischemic stroke. The purpose of this study is to determine plaque morphology and clinical features of symptomatic ICS with non-high intensity signal.
Methods: T1 weighted carotid MRI as well as head MRI was performed in 17 consecutive patients with symptomatic ICS patients. The stenosis rate, the length of the lesion, the existence of major ulcer, the prevalence of diabetes mellitus and ischemic heart disease were obtained.

Results: 6 patients with non-high intensity signal (5 males $65.8 \mathrm{y})$ and 11 patients with high intensity signal $(10$ males $71.7 \mathrm{y})$ were included. With respect to age, sex and the prevalence of diatetes mellitus no signify differences were found. In the non-high signal group, the stenosis rate was significantly higher $(91 \pm 4.7 \%, 70 \pm 18 \%$ respectively, $\mathrm{p} 0.0108)$ and the prevalence of ischemic heart disease was significantly low. (1 of 6,8 of 11 respectively, $\mathrm{p}=0.0269)$ Although not significantly, the longer lesion ( 3.3 $\pm 1.8 \mathrm{~cm}, 2.4 \pm 1.3 \mathrm{~cm}$ respectively) and major ulcer tend to be found more frequently ( $33 \%$ and $18 \%$ respectively) in the non-high intensity group Conclusion: This study may suggest that symptomatic ICS with non-high intensity signal possibly relevant to other mechanism than plaque prolapse in the case of high intensity signal when it causes ischemic stroke.

3-0.4

Category: Head \& Neck

Presentation Type : Oral Presentation

\section{Keywords :}

\section{The Role of Imaging in Perineural Tumoral Spread Detection}

\section{Sladjana Petrovic ${ }^{1}$}

Clinical Centre, Faculty of Medicine, University of Nis, Department of Radiology, Nis-Serbia ${ }^{1}$

The purpose of this study is to review the imaging characteristics of perineural tumoral spread in patients with head and neck tumors.

In this retrospective study, the patient population was composed of 55 male and 30 female patients (mean age 48 years) with head and neck tumors. Patients were examined by MDCT and MRI. Imaging studies depicted the location and extent of tumor involvement, helped determinination of the type of head and neck tumor and their staging. Twenty patients had the signs of perineural tumoral spread on MR or MDCT imaging. The method of choice for diagnostics of perineural spread is MRI which can determine the spread of neural foramen, voluminosity of affected nerve, postcontrast increase of nerve signals, obliteration of surrounding fat and nuscle denervation atrophy. MSCT can also provide useful information about perineural spread relating to spread of neural foramen on skull base and obliteration of fat spaces. Muscle denervation atrophy may also be the consequence of perineural spread of tumor. Imaging plays an important role in detection of perineural tumour spread and good understanding of anatomy of cranial nerves is required. Recognition of radiological signs of perineural spread of head and neck tumour, as well as muscle denervation changes is necessary in order to establish the right diagnosis and determine adequate oncological treatment.

\section{4- Spine}

4-0.1

Category: Spine

Presentation Type : Oral Presentation

Keywords : Craniovertebral junction, MRI, CT, atlas, occiput, dens

Craniovertebral junction congenital and acquired anomalies

Gulgun Yilmaz Ovali ${ }^{1}$, Fatih Duzgun ${ }^{1}$, Serdar Tarhan ${ }^{2}$, Yuksel Pabuscu ${ }^{1}$ Celal Bayar University Medicine School Radiology Manisa-Turkey ${ }^{1}$ Celal Bayar University Medicina School Radiology Manisa-Turkey ${ }^{2}$ 
Craniovertebral junction congenital and acquired anomalies

Learning Objectives: In this educational exhibit we aimed to define the most common developmental and acquired craniovertebral junction abnormalities.

Background: Craniovertebral junction consists of the occipital bone, foramen magnum, clivus, atlas, axis and surrounding ligaments. Pathologies can be congenital, development or acquired.

Conclusion: In this pictorial rewiev we will discuss CT and MRI findings of craniovertebral junction abnormalities and define the origin as congenital diseases, metabolic disorders, inflammatory diseases, trauma, tumours, and infections.

\section{4-O.2 \\ Category: Spine \\ Presentation Type : Oral Presentation}

Keywords : spinal cord, diffusional kurtosis, PSIR

Integration of Phase Sensitive-Inversion Recovery (PSIR) and Diffusional Kurtosis Imaging (DKI) sequences of the spinal cord

Valentina Panara ${ }^{1}$, Eleonora Piccirilli ${ }^{1}$, Loris Di Clemente ${ }^{1}$, Peter Angelo Mattei ${ }^{2}$, Riccardo Navarra ${ }^{1}$, Massimo Caulo ${ }^{1}$

ITAB, Institute for Advanced Biomedical Technologies Department of Neuroscience and Imaging. University "G. d'Annunzio" Chieti. ITALY Chieti-Italy ${ }^{1}$ Ophthalmology Clinic Department of Medicine and Aging Science, G. d'Annunzio University of Chieti-Pescara, 65100 Chieti, Italy Chieti- $^{2}$

Purpose: To determine DKI parameters for white matter (WM) and gray matter (GM) of the cervical spinal cord of healthy subjects by integration with a high contrast GM/WM sequence (PSIR).

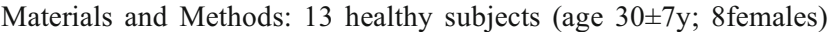
underwent cervical 3TeslaMRI

Scan protocol:

- $\quad$ TSE T2-sagittal (TR3500, TE120 ms, FOV160x250x36, $3 \mathrm{~mm}$ ),

- DKI axial (SE EPI TR3261, TE136 ms, b factors 0,700,2100, FOV128x64x64, 32 directions),

- $\quad$ 3D TFE PSIR axial: TR8, TE4ms, flip angle10 , FOV128X64X64, TFE invert pre-pulse $400 \mathrm{~ms}$.

In four subjects a retest was performed after 1 month.

After PSIR and DKI co-registration, WM and GM were manually segmented in three different levels on the PSIR by two-different experienced neuroradiologists.

The following parameters were calculated in each ROI: mean Kurtosis (MK), axial Kurtosis (AK), radial Kurtosis (RK), fractional anisotropy (FA), mean diffusivity (MD), axial diffusivity (AD), radial diffusivity (RD).

SPSS software and an alpha level of 0.05 were used for statistical analysis.

Results: No significant differences were found in DKI parameters among the three levels $(p=.34)$. For all ROIs in each level MK, AK and RK were significantly higher in GM compared to WM $(p<.01)$. Our results in MK were significantly different from those reported in literature $(\mathrm{p}<.05)$. ICC between the two observers was very high for all comparisons (ICC $>.8$; $\mathrm{p}<.01$ ).

Conclusions: DKI/PSIR integration quantified the microstructure of the cervical spinal cord in healthy subjects in a reliable and reproducible manner. The technique will require further evaluation in patients with spinal cord disease.
4-0.3

Category: Spine

Presentation Type : Oral Presentation

Keywords : Pperipheral nerve pathology, ultrasound, MR

\section{Imaging of peripheral nerve pathology}

$\underline{\text { Daliborka Marić }^{1}}$ - Svjetlana Jefić $^{1}$, Ostoja Savić ${ }^{2}$

University Clinical Centre of the Republic of Srpska Department of Clinical Radiology Banja Luka-Bosnia and Herzegovina ${ }^{1}$ University Clinical Centre of the Republic of Srpska Department of Neurosurgery Banja Luka- ${ }^{2}$

Most common pathology that affects the peripheral nerves is due to compression, trauma or tumor.

Clinical examination and electrophysiologic studies are the traditional mainstay of diagnostic work-up; however, ultrasonography and magnetic resonance imaging provide spatial information regarding the affected nerve and its surroundings, often assisting in narrowing the differential diagnosis and guiding treatment.

Imaging findings of peripheral nerve pathology include enlargement or attenuation, the presence of vascularity, changes of the echogenicity or of the signal of the nerves as well as detecting extraneural causes of compression. MRI is also ideal for imaging allocation of acutely or chronically denervated muscles, thus giving clues for the peripheral nerve affected. It must be stressed that moderately high T2 signal is a normal finding, while on US peripheral nerves exhibit a striated appearance.

We present characteristic cases of peripheral nerve pathology and illustrate and discuss the imaging findings depicted by ultrasound as well as MRI. Radiologists should be aware of the MRI appearance of injury-related neuromas for appropriate diagnosis and avoid misinterpretation as true neoplasms.

\section{4-0.4}

Category: Spine

Presentation Type : Oral Presentation

\section{Keywords :}

\section{Feasibility of Synthetic MRI for the spine}

Maria Isabel Vargas ${ }^{1}$, Bénédicte Delattre ${ }^{2}$, José Boto ${ }^{1}$, Amine Korchi ${ }^{1}$, Karl-Olof Lövblad , Sana Boudabbous ${ }^{2}$

Geneva University Hospital Department of Neuroradiology GenevaSwitzerland ${ }^{1}$ Geneva University Hospital Department of Radiology Geneva-Switzerland ${ }^{2}$

Objective: The goal of this study is to evaluate the feasibility of using one single MRI sequence to obtain all desired turbo spin echo contrasts (T1, T2, STIR, DP) for the evaluation of the spine. This new sequence "Syntac" has already been evaluated for the brain but its added value in spine MR is still unknown. Material and Methods: 20 patients with degenerative, inflammatory and tumoral pathologies were studied with the Syntac sequence, which was compared to conventional sequences on a 1.5T Philips Ingenia system MR. Syntac is a turbo spin echo sequence acquired with different echo and inversion times allowing, in combination with the SyMRI ${ }^{\circledR}$ package, quantitative T1, T2 and DP measurements. From these, any TSE contrast can be generated (synthetic T1, T2, DP, STIR, FLAIR).

Results \& Discussion: All signal anomalies visible on classical T1, T2 or STIR sequences were also visible on the synthetic images. This single sequence acquired in $5 \mathrm{~min} 40 \mathrm{sec}$ can replace at least 3 sequences $(\mathrm{T} 1, \mathrm{~T} 2$, STIR), usually needing $13 \mathrm{~min} 30 \mathrm{sec}$, and offers the possibility to generate other contrasts if required. The advantage of obtaining quantitative measurements is that it provides image information that is completely 
independent of the system used and the specific parameters of each institution. A current limitation of Syntac is a lower signal to noise ratio. Conclusion: The Syntac sequence is feasible for spine MR, and we hope that it could replace conventional sequences in the future.

\section{4-0.5 \\ Category: Spine \\ Presentation Type : Oral Presentation}

Keywords : Spinal Fusion; Postoperatives Complications.

\section{Imaging of Spine Fusion: Post Surgery Complications}

$\underline{\text { Eduardo Bravo }}^{1}{ }_{-}$, Francisca Montoya ${ }^{1}$, Felipe De La Vega ${ }^{1}$

Instituto de Neurocirugía Dr. Asenjo Servicio de Neurorradiología Santiago-Chile ${ }^{1}$

Lumbar spinal fusion has been performed since 1911 to prevent motion of a single or multiple spinal segments, to alleviate pain or to prevent neurologic compromise. A new neurologic deficit, worsening pain or signs of infection may draw attention to a possible postoperative complication. Our main objective is to describe some of the complication that may arise after lumbar spine fusion surgery.

We searched our files, computed tomography (CT) and magnetic resonance imaging (MRI) records in order to find the most exemplifying complication after spine surgery.

There are many different techniques to achieve spinal fusion, each with different types of complications. We present hardware complications such as broken screws, broken bars, lucency changes surrounding the hardware, ejected cage, cage infections, pseudoarthrosis, root fibrosis, secondary spondylolysis or spinous process fracture, bad hardware positioning, and also a case of postoperative esophageal fistula.

Spinal surgery may produce various complications, which we need to be aware of. Timing of surgery, type of surgical procedure and presenting clinical details are fundamental. CT and MRI imaging are the main way to study these patients in order to identify out the surgical complication. MRI is the imaging of choice for the evaluation of abnormal soft tissue or fluid collections. CT is the best imaging method for bone visualization and will give an accurate evaluation of all the metallic hardware used in spinal surgery. The radiologist must be aware of the different complications that may arise from the different hardware and techniques used to achieve spinal fusion.

\section{4-0.6}

Category: Spine

Presentation Type : Oral Presentation

\section{Keywords :}

\section{The Value of Axial Loading in MRI of Lumbar Spine}

$\underline{\text { Deniz Bulja }}^{1}$, Adnan Sehic ${ }^{2}$, Edin Avdagic ${ }^{1}$

Clinical Center University of Sarajevo Neuroradiology Sarajevo-Bosnia and Herzegovina ${ }^{1}$ Clinical Center University of Sarajevo Radiology Sarajevo-Bosnia and Herzegovina ${ }^{2}$

Lumbar spine MRI is one of the most frequent examinations in radiology. A significant number of patients with clinical symptoms of low back pain do not have corresponding imaging abnormalities that would explain severity of their symptoms.

Since symptoms are usually induced or exaggerated by standing or walking, ideal imaging would require standing rather than supine position.
To stimulate upright position we have performed imaging of lumbar spine in 9 patients (33.3\% female, 66.7\% male, mean age 51.55 (36-66) before and after of applying axial loading (DynaWell L-spine; Billdal, Sweden). Lumbar MRI (1.5T) included sagittal, and axial T1w, T2w, and T23D CISS before and after application of axial loading of $50 \%$ of the patient weight. All patients tolerated the study without the need for administration of additional pain medication.

Radiological Interpretation: Radiologist measured intervertebral disk space height of L3/L4, L4/L5, and L5/S1 along with respective area of disk and area of thecal sac before and after application of axial load.

Results: After application of axial loading a mean decrease in disk height was $11 \%, 8.6 \%$, and $13.8 \%$ for $\mathrm{L} 3 / \mathrm{L} 4, \mathrm{~L} 4 / \mathrm{L} 5$, and $\mathrm{L} 5 / \mathrm{S} 1$ respectively. There was an increase of disk area of $4.8 \%, 5.3 \%$, and $3.6 \%$ for $\mathrm{L} 3 / \mathrm{L} 4$, $\mathrm{L} 4 / \mathrm{L} 5$, and L5/S1 respectively. There was a decrease of thecal sac area of $11.8 \%, 19.6 \%$, and $15.9 \%$ for $\mathrm{L} 3 / \mathrm{L} 4, \mathrm{~L} 4 / \mathrm{L} 5$, and $\mathrm{L} 5 / \mathrm{S} 1$ respectively. Conclusion: Lumbar spine MRI with axial loading adds more information about disk height, disk area, and thecal sac indentation which can explain patients' symptomatology and significantly change the treatment plan.

\section{5 - Pediatric}

\section{5-O.1}

Category: Pediatric

Presentation Type : Oral Presentation

Keywords : Brain, Fetal anomalies, Magnetic resonance imaging, Postmortem

Post-mortem High Resolution (HR) magnetic resonance imaging (MRI) in fetuses with brain abnormalities and confirmation of in utero MRI findings

Elisa Scola ${ }^{1}$, Sophie Lombardi ${ }^{2}$, Sabrina Avignone ${ }^{1}$, Claudia Maria Cinnante ${ }^{\top}$, Simona Boito ${ }^{3}$, Faustina Lalatta ${ }^{4}$, Tommaso Rizzuti ${ }^{5}$, Roberto Fogliani ${ }^{3}$, Nicola Persico ${ }^{3}$, Fabio Triulzi ${ }^{1}$

Fondazione IRCCS Ca Granda Ospedale Maggiore Neuroradiology Milano-Italy ${ }^{1}$ Università degli Studi Milano Bicocca Radiology Milano-Italy ${ }^{2}$ Fondazione IRCCS Ca Granda Ospedale Maggiore Obstetrics and Gynecology, Milano-Italy ${ }^{3}$ Fondazione IRCCS Ca Granda Ospedale Maggiore Medical Genetics Unit Milano-Italy ${ }^{4}$ Fondazione IRCCS Ca Granda Ospedale Maggiore Anatomical Pathology Milano-Italy ${ }^{5}$

Objectives: To describe the role of HR post-mortem MRI (HRpmMRI) in validating in utero fetal MRI findings for fetal brain abnormalities.

Methods: Eleven HRpmMRI of unfixed fetal brain[19 post-conceptual weeks (pcw)- $22 \mathrm{pcw}+5$ days (d)] were performed on a 3 Tesla scan within 30 hours from pregnancy termination.Axial, coronal and sagittal T2 weighted

HR images (voxel size $0.3 \times 0.3 \times 1.2 \mathrm{~mm}$ ) and $\mathrm{T} 1$ isotropic weighted images (voxel size $0.73 \mathrm{~mm}$ ) were acquired. All fetuses were previously studied with in utero fetal MRI at $1.5 \mathrm{~T}$ and later underwent the conventional autopsy. Results: In five cases the HRpmMRI confirmed the diagnosis of in vivo brain fetal MRI (cerebellar hypoplasia,romboencephalosynapsis, syntelencephaly, agenesis of corpus callosum (cc) and Chiari II malformation).

In six cases it added diagnostic informations: in four cases it ruled out additional brain malformations that may be associated to main in vivo brain MRI findings (septo-optic dysplasia in two cases of agenesis of septum pellucidum,interhemispheric cyst in one case of partial cc agenesis with cortical malformation, "joubert syndrome plus" findings and supratentorial malformations in one case of molar tooth malformation); in one case of cerebellar hypoplasia it demonstrated olivary nuclei hypoplasia and in one case of brainstem malformation it depicted a mesencephalic diencefalic junction anomaly. 
Conclusions: The HRpmMRI can be a convincing tool to validate the in utero fetal brain MRI findings in fetal brain abnormalities, considering that the diagnostic accuracy of in vivo brain fetal MRI can be limited by the early gestational age.

\section{5-0.2}

Category: Pediatric

Presentation Type : Oral Presentation

\section{Altered BOLD Signal Following Mirror Therapy on task-based fMRI in Children with Hemiparetic Spastic Cerebral Palsy}

$\underline{\text { Arzu Ceylan Has }}{ }_{-}{ }^{1}$, Duygu Korkem ${ }^{2}$, Mintaze Kerem Gunel ${ }^{3}$, Kader K. Oguz ${ }^{4}$

National Magnetic Resonance Research Center, UMRAM, Bilkent University National Magnetic Resonance Research Center Ankara-Turkey ${ }^{1}$ School of Pyhsical Therapy and RehabilitationTurgut Ozal University School of Pyhsical Therapy and Rehabilitation Ankara-Turkey ${ }^{2}$ Department of Physical Therapy and Rehabilitation, Hacettepe University Department of Physical Therapy and Rehabilitation Ankara-Turkey ${ }^{3}$ Department of Radiology, Hacettepe University Department of Radiology Ankara-Turkey ${ }^{4}$

Aim: To study and compare brain activation during motor task in children with hemiparetic spastic cerebral palsy (HSCP) in pre- and postrehabilitation periods.

Materials and Methods: Ten children (mean \pm SD: $11.88 \pm 2.84$ years, F/M: $8 / 2$ ) with diagnosis of HSCP on basis of clinical, electrophysiological and MRI findings were studied. All patients had pre- and post-rehabilitation (mirror therapy, applied 8 weeks, 3-times per week 30-minutes-long) imaging with the same protocol (multi-slice single-shot $\mathrm{T} 2 *$-W echoplanar and structural-3D T1-W sequences) on the same 3T MR scanner. Post-rehabilitation studies were performed at 2 months upon completion of rehabilitation. fMRI-BOLD applied cycles of $20 \mathrm{sec}$ alternating right and left hand-movement and $20 \mathrm{sec}$ rest, repeated 6 times.

Analysis of the fMRI data was performed using a standard procedure provided by the BrainVoyager QX 1.2 (www.BrainVoyager.com). Correlation estimation and correction for multiple comparisons was done $(\mathrm{p}<0.05, \mathrm{t}>3.1$, minimum cluster threshold: $10 \mathrm{~mm}$ ). Region of interest (ROI)s were created by Brodmann area (BA) 4 and BA6 cortex activations and averaged $t$-stat value of each ROI was extracted.

Results: The patients showed clinical improvement following rehabilitation (WeeFIM; $\mathrm{p}<0.05$ ). We found significantly increased activation in bilateral BA4 and BA6 in each patient after the rehabilitation.

Conclusion: Along with clinical improvement in children with HSCP, increased BOLD signal alteration was observed in primary motor and premotor cortices suggesting that rehabilitation effects on the brain may be assessed by using fMRI.

\section{5-O.3}

Category: Pediatric

Presentation Type : Oral Presentation

\section{MRI findings after surgery for congenital heart disease: insight from} brain imaging

Cristina Utrilla ${ }^{1}$, Arantxa Royo ${ }^{1}$, Alberto Alvarez-Muelas ${ }^{1}$, Amelia Fernandez-Zubillaga ${ }^{1}$, Diego Garrido ${ }^{1}$, Gonzalo Garzon ${ }^{2}$

Hospital Universitario La Paz Neuroradiology MADRID-Spain ${ }^{1}$ Hospital Universitario La Paz Radiology MADRID-Spain ${ }^{2}$

Congenital heart disease is a common cause of childhood morbidity, occurring in 6-8/1000 live births, up to $50 \%$ of these children requiring open-heart surgery to correct their defect. Neurological injury is a major and often debilitating complication of congenital heart disease and open- heart surgery. The most common complications of this surgery involve the central nervous system.

We reviewed the brain MRI images of 42 infants (in the first 6 months of live) that were examined after having undergone cardiac surgery in our institution during 18 months.

The aim of this review was to document the rate of neurological complications visible in MRI. 22 patients showed intracranial bleeding (most of them, minimal hemosiderin deposits in T2* sequences), 8 patients had hypoxicischemic findings, and 9 patients had other findings (benign enlargement of the subarachnoid space, cortical retraction, indirect signs of infection). Four of the patients showed more than one type of findings (stroke + bleeding, bleeding + retraction). The brain MRI was normal in 7 cases.

The goal of this exhibit is to describe the most common findings in brain MRI in newborns after cardiac surgery.

5-0.4

Category: Pediatric

Presentation Type : Oral Presentation

Keywords : Pediatrics, head and neck neoplasms, ultrasonography, magnetic resonance imaging

\section{A practical clinical and imaging approach to pediatric neck masses}

Yeliz Pekcevik ${ }^{1}$, Hilal Sahin ${ }^{1}$, Orhan Dogan ${ }^{1}$, Handan Guleryuz ${ }^{2}$, Ilker Burak Arslan $^{3}$, Sureyya Hikmet Kozcu ${ }^{3}$, Ibrahim Cukurova ${ }^{3}$

Tepecik Training and Research Hospital Department of Radiology IzmirTurkey ${ }^{1}$ Dokuz Eylül University School of Medicine Department of Radiology Izmir-Turkey ${ }^{2}$ Tepecik Training and Research Hospital Department of Otorhinolaryngology-Head and Neck Surgery Izmir-Turkey ${ }^{3}$

Objective: Neck masses are common clinical question in children and can be a diagnostic challenge. Careful consideration of clinical history and accurate physical evaluation is mandatory in evaluation. Clinical assessment guides the use of diagnostic imaging technique. The accurate interpretation of the multimodality imaging is very important and affects the clinical approach. We aim to provide a practical imaging approach to pediatric neck masses. Material and Methods: The clinical presentation and the sonography, Doppler sonography, computed tomography, magnetic resonance imaging or magnetic resonance angiography findings in 250 children with neck masses were evaluated.

Results: The most distinguished clinical and imaging features of the common and rare neck masses were demonstrated and discussed with a proposed practical imaging approach.

Conclusion: Pediatric neck masses are common diagnostic problem. Careful attention to the clinical findings as well as the clues obtained from the multimodality imaging can provide accurate diagnosis and appropriate management.

5-0.5

Category: Pediatric

Presentation Type : Oral Presentation

Keywords : fetal MRI, diffusion tensor imaging, tractography, in utero DTI, fiber pathways

Mapping the fetal brain connectome with high angular resolution diffusion tensor imaging

Andras Jakab ${ }_{-}{ }_{-}$, Ruth Tuura-O ${ }^{1}$, Christian Kellenberger ${ }^{2}$, Ianina Scheer ${ }^{2}$ University Children Center for MR-Research Zürich-Switzerland ${ }^{1}$ University Children Department of Diagnostic Imaging ZürichSwitzerland $^{2}$ 
Our purpose was to demonstrate the feasibility of a novel, high angular resolution in utero DTI based tractography in reconstructing the wholebrain fiber anatomy in a selected cohort of fetuses with unaffected and pathological brain development.

To evaluate the effect of image sequence and scanner parameters on the reconstructed fiber tracts, we used DTI with varying parameter sets. Images for 8 fetuses (gestational age: 23-30. week, normal neurodevelopment: 4 cases, pathological development, 4 cases) were acquired on $1.5 \mathrm{~T}$ or $3.0 \mathrm{~T}$ MRI using repeated DTI sessions resulting in 45 to 90 diffusion weighting directions, $\mathrm{b}=700 \mathrm{~s} / \mathrm{mm}^{2}$. Motion-corrected and isotropically re-sampled data were used to estimate diffusion tensors and to initiate streamline tractography over the entire brain volume.

We were able to reconstruct the 3D structure of fiber pathways using isotropically re-sampled diffusion tensor imaging data. This superior longitudinal fascicle, the Probst bundle, the inferior fronto-occipital fascicle could be mapped for the majority of fetuses in our work. Interestingly, the arcuate fascicle, which is considered an important fiber pathway for language functioning, was also recognized in some fetuses.

Repeated sessions - yielding optimally 45 diffusion-weighting directions can be used to overcome to limitation of motion corrupted data during fetal DTI. This technique allowed motion-correction, motion censoring, while the resampling to isotropical 3D space allows the reconstruction of finer-scale anatomical fibers, such as the association fibers. Recognizing such fibers in utero and measuring their microstructural properties with DTI with high angular resolution can be an important marker of neurodevelopment in the future.

\section{5-0.6}

Category: Pediatric

Presentation Type : Oral Presentation

\section{Keywords :}

Dural Arteriovenous Fistulas in children with Vein of Galen Malformation - The role of treatment timing and embolic material

$\underline{\text { Dan Meila }}{ }^{1}$, Cynthia Schmidt ${ }^{1}$, Katharina Melber ${ }^{1}$, Dominik Grieb ${ }^{1}$, Björn Greling ${ }^{1}$, Heinrich Lanfermann ${ }^{2}$, Friedhelm Brassel ${ }^{1}$

Sana Kliniken Duisburg Department of Radiology and Neuroradiology Duisburg-Germany ${ }^{1}$ Medical School Hannover Department of Diagnostic and Interventional Neuroradiology Hannover-Germany ${ }^{2}$

Introduction: The association of dural arteriovenous fistulas (DAVF) in children with Vein of Galen Malformation (VGM) has recently been reported. In a larger series of cases treated with transarterial NBCA embolization $30 \%$ had DAVF. We wanted to analyse the development of DAVF in our cohort of children with VGM and to evaluate whether their occurrence depends on different treatment options and embolic materials.

Methods: We analysed 43 VGM cases treated with a combined transarterial and transvenous approach between 2003 and 2016. In our early series until 2012, we used coils solely in 21 children. Since 2012, 22 children were treated with the combination of coils and Onyx.

Results: In our early series treated with coils solely no case presented initially with or developed DAVF over time on follow-up angiograms. In our recent series we found four cases $(9 \%)$ with DAVF. In two patients (5\%), DAVF were found on initial angiogram. Both patients presented to our department at the age $>2$ years and were not treated before elsewhere. One patient $(2 \%)$ presented to our department with too proximal occlusion of arterial feeder performed in another institution. Only one patient (2\%) developed DAVF in our department after the use of transarterial Onyx. Interestingly, this child did not develop DAVF as long as we used coils solely and the localization of his DAVF was exactly where an Onyx cast was found, too.

Conclusion: Treatment timing, option and choice of embolic material might have a considerable impact on the occurrence of DAVF in VGM.
POSTER ABSTRACTS

\section{1 - Diagnostic Brain}

\author{
1-P.01 \\ Category: Diagnostic Brain \\ Presentation Type : Poster Presentation
}

Keywords : Cerebral arterial variation, Anterior cerebral artery, MR angiography

Carotid-Anterior Cerebral Artery Anastomosis Diagnosed by $\mathrm{Mr}$ Angiography

Akira Uchino ${ }_{-}^{1}$, Naoko Saito ${ }^{1}$

Saitama Medical University International Medical Center Diagnostic Radiology Hidaka-Japan ${ }^{1}$

Purpose: Carotid-anterior cerebral artery (ACA) anastomosis or an infraoptic course of the ACA is rare, with reported prevalence of about $0.1 \%$. In this variation, the ACA arises from the ophthalmic segment of the internal carotid artery, courses cranially through the space between the optic nerves, and anastomoses with the A1-A2 junction of the ACA. We evaluated its features on magnetic resonance (MR) angiography.

Methods: From our university hospital records, we identified 12 patients with this cerebral arterial variation that was diagnosed by MR angiography at 1.5 or 3.0 tesla machine using the standard 3-dimensional time-offlight technique. We checked source images of MR angiography to confirm the variation and created partial maximum-intensity-projection images of the carotid system to demonstrate the variations clearly.

Results: Seven of the 12 cases were right-sided, two were left-sided, and three were bilateral. In the 7 right-sided cases, the A1 segment of the ipsilateral ACA was absent in four, normal in two, and tiny in one case. In all 3 bilateral cases, the bilateral A1 segments were absent. Of 14 ipsilateral ophthalmic arteries (OAs), three arose from the middle meningeal artery and two from the anastomotic artery.

Conclusion: We reaffirmed the right-side predominance of this variation. There is wide spectrum in this variation. Bilateral type is considered most severe, but it may easily be overlooked because of its symmetry. Associated $\mathrm{OA}$ variations are frequently observed.

\section{1-P.02 \\ Category: Diagnostic Brain \\ Presentation Type : Poster Presentation}

Keywords : hemorrhage, corpus callosum, magnetic resonance imaging, anoxic brain damage

Microhemorrhages in the corpus callosum as the distinctive anoxic brain injury

$\underline{\text { Dong Woo Park }}{ }^{1}$, Tae Yoon Kim ${ }^{1}$

Hanyang University Guri Hospital Radiology Guri-Korea, South ${ }^{1}$

Purpose: Vasogenic edema in the corpus callosum is reported to be a characteristic finding in severe acute respiratory distress including high-altitude cerebral edema (HACE). Furthermore, microhemorrhages in the corpus callosum have been found at autopsies in HACE victims. This study is to present microhemorrhages in the corpus callosum in brain MR images after suffering from anoxic brain injury.

Materials and Methods: Among 16 patients who survived from anoxic brain injury and conducted MR imaging, susceptibility weighted image (SWI) of five patients who show microhemorrhages in the corpus callosum are evaluated. Results: SWI reveals multiple microhemorrhages, predominantly in the corpus callosum in all five patients who suffered from anoxic brain injury. An 
identical pattern of microhemorrhages has previously been described in climbers who suffered from high altitude cerebral edema (HACE). The incidence of microhemorrhage to be detected on SWI is about $31 \%$. Conclusions: This study implies that anoxic brain injury show congruent cerebral injuries as high-altitude cerebral edema. Microhemorrhages in the corpus callosum could be suggestive MR findings that characterized as anoxic brain injury. It may be developed by disruption of the bloodbrain barrier due to increased capillary and central venous pressures from 'anoxic brain injury' induced hypoxia

\section{1-P.03 \\ Category: Diagnostic Brain \\ Presentation Type : Poster Presentation}

\section{Keywords :}

Paraneoplastic limbic encephalitis, an uncommon presentation of breast cancer: case report and discussion

$\underline{\text { Yon-Kwon Ihn }}{ }^{1}$, Won-Sang Jung ${ }^{1}$, Min Kyung Jung ${ }^{1}$

St.Vincent's hospital Radiology Suwon-Korea, South ${ }^{1}$

Background: Paraneoplastic neurological disorders (PND) are defined as remote effects on the nervous system that are not caused directly by the tumor, its metastases, or metabolic disruptions. This syndrome occurs in less than 1 per 10,000 patients diagnosed with a malignancy. Many antibodies are found in the central nervous system in PND, the most well known are Anti$\mathrm{Hu}, \mathrm{Tr}, \mathrm{CV} 2 \mathrm{Ta}$, Yo, Ri and amphiphysin. Paraneoplastic limbic encephalitis occurs due to involvement of the limbic system secondary to an autoimmune response to neurons of the brain provoked by the antibodies. Patients, thus, present with seizures, changes in mood, memory, and personality.

Case Report: Forty-seven years-old female patient presented with impaired recent memory impairment and diplopia. Laboratory workup was not remarkable for herpes simplex virus. Anti-Hu, anti-Ma and NMDA-receptor antibodies were requested. Further workup included brain magnetic resonance imaging (MRI), and MR spectroscopy, which suggested a diagnosis of encephalitis for limbic encephalitis. Chest CT revealed a well enhancing mass in the right breast and enlarged axillary lymph node. Mammography and breast ultrasonography showed fine pleomorphic calcific mass. US guided breast biopsy was obtained which confirmed the diagnosis of breast cancer.

Conclusions: A very high index of suspicion should thus be present when patients present with paraneoplastic abnormalities. It must be emphasized that limbic encephalitis (LE) occurs at an early stage of the disease development and therefore the detection of paraneoplastic LE can lead to a quicker identification of the underlying malignancy and a better outcome.

\section{1-P.04}

Category: Diagnostic Brain

Presentation Type : Poster Presentation

Keywords : Arterial transit artifact. Arterial spin labeling. Cerebrovascular reserve Carotid occlusive disease

Correlation of cerebrovascular reserve assessed by acetazolamidestress SPECT with collaterals on arterial spin-labeling MRI in patients with carotid occlusive disease

$\underline{\text { Hyunkoo Kang }}$ - $^{\text {I }}$

Seoul Veterans Hospital Department of Radiology Seoul-Korea, South ${ }^{1}$

Purpose: We evaluated the correlation between cerebrovascular reserve (CVR) on acetazolamide-stress single photon emission computed tomography (SPECT) brain scans and collaterals on arterial spinlabeling (ASL) magnetic resonance imaging (MRI) in internal carotid artery (ICA) stenosis.

Materials and Methods: 74 patients with ICA stenosis $(>70 \%)$ underwent cerebral blood flow (CBF) examinations by pulsed ASL brain MRI and SPECT imagings with Tc-99m HMPAO in the resting and after acetazolamide challenge. We observed the presence of intracranial collaterals, which are manifested by arterial transit artifact, on ASL brain perfusion scan. CVR based on rest-SPECT and acetazolamide-stress SPECT was calculated. With acetazolamide stress SPECT, the 74 patients were grouped as either showing or not showing evidence of decreased CVR. We assessed the relationship between reduced CVR and intracranial collaterals shown as ATA on ASL brain perfusion.

Results: In 17/74 (23\%) of the ICA stenosis patients, ASL showed ATA in ipsilateral to the stenosis. With acetazolamide stress SPECT, the 21/74 (28\%) patients showed evidence of decreased CVR. In 7/53 (13\%) of the normal CVR group and 10/21 (48\%) of the reduced CVR from the SPECT results, pulsed ASL showed ATA in ipsilateral to the stenosis. Significant relationship was observed between reduced CVR group and ATA showing group in ICA stenosis patients on ASL brain perfusion $(\mathrm{p}=0.004)$.

Conclusion: Our results suggest that ICA stenosis patients who had reduced CVR on acetazolamide-stress SPECT brain scans showed increased dependence on intracranial collaterals shown as ATA on ASL brain scans.

\section{1-P.05}

Category: Diagnostic Brain

Presentation Type : Poster Presentation

\section{Keywords :}

\section{Arterial spin labeling in acute seizure}

Mathieu Schertz ${ }^{1}$, Samia Belkacem ${ }^{1}$, Nadya Pyatigorskaya ${ }^{1}$, Baronnet Flore $^{2}$, Dormont Didier ${ }^{1}$, Delphine Leclercq ${ }^{1}$, Bruno Law-ye ${ }_{-}^{1}$

APHP - Hospital Pitié-Salpêtrière Neuroradiology Paris-France ${ }^{1}$ APHP Hospital Pitié-Salpêtrière Neurology Paris-France ${ }^{2}$

Purpose: Seizure activity can cause neurological deficit and mimic acute stroke. Arterial spin labeling (ASL) is a noninvasive way to measure cerebral blood flow. Its utility has never been studied in this indication. The objective of this study was to evaluate the characteristics of ASL imaging in acute seizure as well as its sensitivity and diagnostic performance.

Materials and Methods: Among a cohort of patients presenting with a brutal neurologic deficit addressed for suspicion of stroke in a specialized national stroke center, we retrospectively reviewed 24 cases of stroke-like proven seizures who underwent magnetic resonance imaging (MRI) with ASL perfusion at acute phase.

Results: All patients presented ASL hyperperfusion in the epileptogenic cortical region with a good correlation to electroencephalography and clinical findings. Additionally, 7 patients had a hyperperfused area in the homolateral pulvinar, and 5 patient had a hyperperfused area related to cerebellar diaschisis. Underlying causing lesions were found in 19 cases (ischemic sequelae $\mathrm{n}=3$, brain tumors $\mathrm{n}=5$, haemoraghe sequeale $\mathrm{n}=3$, others $\mathrm{n}=8$ ). Associated diffusion abnormalities were found in 15 cases. Conclusions: High cerebral blood flow in ASL can be seen in in the cortical epileptogenic zone, in the homolateral pulvinar and in contralateral cerebellum (cerebellar diaschisis) of patients with acute seizure. The mechanism of hyperperfusion due to seizure activity may be related to transient loss of autoregulatory function in the surrounding vasculature. Arterial spin labeling is useful in the differential diagnosis of acute neurologic deficits and is more sensitive than conventional MR sequences. 
1-P.06

Category: Diagnostic Brain

Presentation Type : Poster Presentation

Keywords : Intracranial cysts, Focal epilepsy, Arachnoid cysts

Intracranial Cysts and Focal Epilepsy: Hospital Based Case Control Study

Ivan Nikolic ${ }^{1}$, Aleksandar Ristic ${ }^{2}$, Dusan Damjanovic ${ }^{3}$, Marija Jovanovic , Kristina Davidovic ${ }^{3}$, Marko Dakovic ${ }^{4}$, Ruzica Maksimovic $^{3}$, Dragoslav Sokic ${ }^{2}$

Clinical Center of Serbia, Center for Radiology and MRI, Belgrade-Serbia ${ }^{1}$ Clinical Center of Serbia, School of Medicine, University of Belgrade, Center for Epilepsy and Sleep Disorders, Neurology Clinic, BelgradeSerbia ${ }^{2}$ Clinical Center of Serbia, School of Medicine, University of Belgrade, Center for Radiology and MRI, Belgrade-Serbia ${ }^{3}$ Faculty of Physical Chemistry, University of Belgrade, Belgrade-Serbia ${ }^{4}$

Objective: Intracranial cysts (ICs) are common findings in brain Magnetic Resonance Imaging (MRI). Our aim was to examine frequency and distribution of ICs in patients with focal epilepsy, compared to healthy control subjects, and to investigate the association of different IC types and electro-clinical features of focal epilepsy.

Patients and Method: We performed a retrospective case-control study, using data from 180 patients that underwent video-EEG monitoring at the Epilepsy Center, Neurology Clinic, Clinical Center of Serbia, between 2009 and 2012, and of 114 healthy controlled subjects. Analysis of electro-clinical data and structural MRI images was conducted.

Results: A significantly higher proportion of different ICs in the focal epilepsy group $(55 / 180 ; 31.1 \%)$ compared to healthy control subjects $(16 / 114 ; 14 \%)(\mathrm{p}=0.001)$ was identified. Only arachnoid cysts (AC) and cysts in dysembryoplastic neuroepithelial tumors (DNET cysts) significantly differed in predominance from the control group; AC were found in $19(10.5 \%)$ active, and in $3(2.6 \%)$ control subjects, and DNET cysts in $8(4.4 \%)$ active and none $(0 \%)$ in healthy control subjects. Significant congruence of semiological features or interictal and ictal EEG with arachnoid cyst localization was identified in only one MRI nonlesional patient with temporal cyst localization.

Conclusion: ICs are seen often in patients with focal epilepsy. However, only arachnoid cysts were significant outliers among different IC types when comparing to healthy controls. Explicit association between focal epilepsy and arachnoid cysts is possible but exceptional. More likely, focal epilepsy and arachnoid cysts share a common etiological ancestor but represent distant and distinct entities.

\section{1-P.07}

Category: Diagnostic Brain

Presentation Type : Poster Presentation

Keywords : AVM of the brain, bleeding, morphological parameters

Predictive Mofological Parameters for Bleeding of Brain Arteriovenous Malformations by Multidetector Ct Angiography

Biljana Milatovic ${ }^{1}$, Svetlana Milosevic Medenica ${ }^{1}$, Goran Tasic ${ }^{2}$, Igor Nikolic $^{2}$

Clinical center of Serbia radiology Belgrade-Serbia ${ }^{1}$ Clinical center of Serbia neurosurgery Belgrade-Serbia ${ }^{2}$

Patients with brain arteriovenous malformation (AVM) have a risk to bleed, and the goal of this study is to examine the effect of morphological predictive characteristics of AVM for haemorrhage using multidetector CT angiography (MDCTA).

Material: This is a series of 57 patients with a mean age of 35.46 years, who were diagnosed at the Center for Radiology and magnetic resonance imaging in 5 years period. For all patients, the diagnosis was made using MDCT angiography. Two groups of patients were observed. The first group includes patients who were not initially presented by haemorrhage while the second group was initially presented by haemorrhage. Both groups were treated with the combination of medical therapy with embolization / surgery / radiotherapy.

Results: Deep venous drainage ( $p<0.05$ ), the combined arterial supply from different basins $(\mathrm{p}<0.05)$ with a length $>60 \mathrm{~mm}$, venous dilation present in the drainage vein $(p<0.01)$ and the angle of casting supply arteries in the nidus $(\mathrm{p}<0.01)$, carry a risk of bleeding. In the group of patients who had initial haemorrhage a mean value of the size corner casting was $130 \mathrm{o}$, while the other group mean value of the size of the measured angle was 103.81 with a standard deviation of $17.21(\mathrm{p}<0.01)$.

Conclusion: Arteriovenous malformations with the deep venous drainage from the carotid and VB basin, the length of the feeding arteries in the nidus $\geq 130^{\circ}$ and dilatation and / or venous aneurysm drainage vessel are a predictive model for clinical presenting by haemorrhage.

\section{1-P.08}

Category: Diagnostic Brain

Presentation Type : Poster Presentation

Keywords : Susceptibility-weighted imaging. Magnetic Resonance Imaging. Brain.

Differentiation of glioblastoma multiforme and single brain metastasis by the distribution pattern of intratumoral susceptibility sign derived from susceptibility-weighted imaging

Hyunkoo Kang ${ }^{1}$, Keuntak Roh ${ }^{1}$

Seoul Veterans Hospital Department of Radiology Seoul-Korea, South ${ }^{1}$

Purpose: The aim of this study is to determine whether the distribution pattern of intratumoral susceptibility sign (ITSS) derived from susceptibility-weighted imaging (SWI) could differentiate glioblastoma multiforme (GBM) and single brain metastasis.

Methods: Nineteen intracranial brain neoplasm patients (19 male, age $69.4 \pm 7.1$ years (mean \pm SD), 7 with non small cell lung cancer (NSCLC) metastases, 5 with small cell lung cancer (SCLC) metastases, 2 with renal cell cancer (RCC) metastases, and 5 with glioblastomas WHO IV), underwent examinations that included SWI in addition to conventional magnetic resonance (MR) sequences on a 3T, were enrolled in this study. Two radiologists investigated the distribution patterns of ITSS of the tumors and applied an ITSS grading system based on the degree of the ITSS. Then, we compared the grade of the visibility of ITSS in the central portion of tumors (CITSS) and in the tumor capsular area (PITSS) on SWI in consensus.

Results: The mean visibility scores of the CITSS in GBM and SCLC brain metastases were significantly higher than NSCLC and RCC brain metastases. The mean visibility scores of the PITSS in GBM and SCLC brain metastases were significantly lower than NSCLC and RCC brain metastases ( $p<0.05$, Student t-test).

Conclusion: The findings of our study show that although the SWI was unable to differentiate between GBM and SCLC brain metastasis, differentiation was achieved between GBM and the other brain metastases using the ITSS distribution pattern of the brain tumors. 


\section{1-P.09}

Category: Diagnostic Brain

Presentation Type : Poster Presentation

Keywords : METABOLIC ENCEPHALOPATHY, UREMIA, CHRONIC RENAL FAILURE, CREATININE

\section{Uremic Encephalopathy: MR Imaging findings and Clinical Correlation}

Chang June Song ${ }^{1}$, Dami Kim ${ }^{1}$, Inho Lee ${ }^{1}$, Inkyu Yoo ${ }^{2}$

Chungnam National University Hospital Radiology Daejon-Korea, South ${ }^{1}$ Eulji University Hospital Radiology Daejon-Korea, South ${ }^{2}$

Background and Purpose: Uremic encephalopathy is a metabolic disorder in patients with renal failure. The purpose of this study was to describe the MR imaging findings of uremic encephalopathy.

Materials and Methods: This study retrospectively reviewed MR imaging findings in 10 patients with clinically proved uremic encephalopathy between May 2005 and December 2014. Parameters evaluated were lesion location and appearance; MR signal intensity of the lesions on T1WI, T2WI, and T2 fluid-attenuated inversion recovery images; the presence or absence of restricted diffusion on diffusion-weighted images and apparent diffusion coefficient maps; and the reversibility of documented signalintensity abnormalities on follow-up MR imaging.

Results: MR imaging abnormalities accompanying marked elevation of serum creatinine (range, $4.3-11.7 \mathrm{mg} / \mathrm{dL}$ ) were evident in the 10 patients. Nine patients had a history of chronic renal failure with expansile bilateral basal ganglia lesions, and 1 patient with acute renal failure had reversible largely cortical lesions. Two of 6 patients with available arterial blood gas results had metabolic acidosis.

All basal ganglia lesions showed expansile high signal intensity (lentiform fork sign) on T2WI. Varied levels of restricted diffusion and a range of signal intensities on DWI were evident and were not correlated with serum Cr levels. All cortical lesions demonstrated high signal intensity on T2WI. Four patients with follow-up MR imaging after hemodialysis showed complete resolution of all lesions.

Conclusions: The lentiform fork sign is reliable in the early diagnosis of uremic encephalopathy, regardless of the presence of metabolic acidosis. Cytotoxic edema and/or vasogenic edema on DWI/ADC maps may be associated with uremic encephalopathy.

\section{1-P.10}

Category: Diagnostic Brain

Presentation Type : Poster Presentation

\section{Keywords :}

Prognostic value of preoperative dynamic contrast-enhanced MRI perfusion parameters for high-grade glioma patients

Vasileios K. Katsaros ${ }^{1}$, Agne Ulyte ${ }^{2}$, Evangelia Liouta ${ }^{3}$, George Stranjalis ${ }^{4}$, Christos Boskos ${ }^{5}$, Nickolas Papanikolaou ${ }^{6}$, Jurgita Usinskiene ${ }^{7}$, Sotirios Bisdas ${ }^{8}$

University of Athens MRI - Neuroradiology Pikermi-Rafina-Greece ${ }^{1}$ Vilnius University Faculty of Medicine Vilnius-Lithuania ${ }^{2}$ University of Athens Neuropsychology Athens-Greece ${ }^{3}$ University of Athens Neurosurgery Athens-Greece ${ }^{4}$ University of Athens Radiation Oncology Athens-Greece ${ }^{5}$ Center for the Unknown, Champalimaud Biomedical Engineering LisbonPortugal ${ }^{6}$ National Cancer Institute Radiology Vilnius-Lithuania ${ }^{7}$ National Hospital for Neurology and Neurosurgery, University College London Hospitals Neuroradiology London-United Kingdom ${ }^{8}$
Purpose: The prognostic value of the dynamic contrast-enhanced (DCE) MRI perfusion and its histogram analysis-derived metrics is not well established for high-grade glioma (HGG) patients. The aim of this prospective study was to investigate DCE perfusion transfer coefficient (Ktrans), vascular plasma volume fraction (vp), extracellular volume fraction (ve), reverse transfer constant (kep) and initial area under gadolinium concentration time curve (IAUGC) as predictors of progression free (PFS) and overall survival (OS) in HGG patients.

Methods: Sixty-nine patients with suspected anaplastic astrocytoma or glioblastoma underwent preoperative DCE-MRI scans. DCE perfusion whole tumor region histogram parameters, clinical details, PFS and OS data were obtained. Univariate, multivariate and Kaplan-Meier survival analyses were conducted. Receiver operating characteristic (ROC) curve analysis was employed to identify perfusion parameters with the best differentiation performance.

Results: On univariate analysis, ve, and skewness of $\mathrm{vp}$ had significant negative impacts, while kep had significant positive impact on OS $(\mathrm{P}<0.05)$. ve was also a negative predictor of PFS $(\mathrm{P}<0.05)$. Patients with lower ve and IAUGC had longer median PFS and OS on Kaplan-Meier analysis $(\mathrm{P}<0.05)$. Ktrans and ve could also differentiate grade III from IV gliomas (area under the curve 0.819 and 0.791 , respectively).

Conclusions: High ve is a consistent predictor of worse PFS and OS in HGG glioma patients. vp skewness and kep are also predictive for OS. Ktrans and ve demonstrated the best diagnostic performance for differentiating grade III from IV gliomas.

\section{1-P.11}

Category: Diagnostic Brain

Presentation Type : Poster Presentation

Keywords : basilar artery, CT angiography, non-invasive imaging

\section{Impact of Age and Gender on Basilar Artery Size}

$\underline{\text { Filip Vitosevic }}{ }^{1}$, Milica Kovacevic ${ }^{1}$, Svetlana Milosevic-Medenica ${ }^{1}$

Clinical Center of Serbia Center for Radiology and MRI BelgradeSerbia $^{1}$

Background: Study was performed to provide measurements of the basilar artery using non-invasive imaging.

Methods: 120 patients aged from 12 to 76 years were analyzed using CT angiography, but without any vascular abnormality in the posterior circulation. Length and diameter of basilar artery was measured. Length was measured from vertebro-basilar junction to its terminus. Diameter was measured three millimeters distal to vertebro-basilar junction.

Results: The mean length of basilar artery was $32 \mathrm{~mm}$ (from $20,7 \mathrm{~mm}$ to $48,5 \mathrm{~mm}$ ). Mean length in man was $34 \mathrm{~mm}$, while in woman was $30,8 \mathrm{~mm}$. There was significant difference in basilar artery length between man and woman, man had a longer basilar artery $(\mathrm{p}<0,01)$. The mean diameter of basilar artery was $2,97 \mathrm{~mm}$ (from 1,2 to 5,6 $\mathrm{mm}$ ). Mean diameter in man was $3 \mathrm{~mm}$, while in woman was 2,95 $\mathrm{mm}$. There was no significant difference in basilar artery diameter between genders in our study $(p>0,05)$. There was no significant difference in basilar artery length between patients younger and older than 60 years $(p>0,05)$. But, there was significant difference between these groups in basilar artery diameter. Older patients had statistically larger diameter, mean diameter in older group was 3,3 $\mathrm{mm}$, while in younger was $2,8 \mathrm{~mm}(\mathrm{p}<0,05)$.

Conclusion: Modern non-invasive imaging procedures can provide precise and useful information for vessels analysis. Our Study showed that older patients tend to have larger basilar artery diameter, while man tend to have longer basilar artery than woman. This information may be useful for planning and performing neurointerventional procedures. 
1-P.12

Category: Diagnostic Brain

Presentation Type : Poster Presentation

Keywords :

\section{D Imaging of Intracranial Tumors at 1.5 $\mathrm{T}$ and 3.0 $\mathrm{T}$}

\author{
Andrea Bink ${ }^{1}$, Christoph Stippich $^{1}$
}

Neuroradiology University Hospital Basel Basel-Switzerland ${ }^{1}$

Aim: Report on advantages with the Golden-angle RAdial Sparse Parallel MRI sequence (GRASP) in patients with intracranial tumors. The GRASP sequence combines the techniques of compressed sensing and parallel imaging for rapid continuous acquisition with the advantages of flexible spatiotemporal resolution using the golden-angle radial sampling scheme.

Materials and Methods: The GRASP sequence was implemented on 5 MR scanners. The parameters were: TR/TE 4.42/2.13 msec, image matrix $256 \times 256 \mathrm{~mm}$, voxel size $2.0 \times 1.1 \times 1.1 \mathrm{~mm}$, temporal resolution $3.5 \mathrm{~s}$, TA 4:07 min. Permeability maps were calculated from the GRASP sequence in each patient. Images were read by two neuroradiologists with respect to delineation of pathologies and artifacts. Data were compared to standard 3D MPRAGE post CE.

Results: By now 22 patients were retrospectively included. The reports were as follows: glioma (10x), lymphoma (1x), metastasis $(3 x)$, meningeoma $(5 x)$, vestibular schwannoma $(3 x)$. All pathologies were correctly detected by the readers. Image quality was judged at least comparable to standard 3D T1w images, especially appreciated was the possibility to see distribution of contrast media over time with additional high regional resolution as well as measurement of signal-time-curves and permeability in all pathologies with one sequence. Pulsation artifacts were not observed.

Conclusions: With the GRASP sequence all intracranial tumors were well evaluable by providing high temporal, high spatial resolution and permeability maps. Gy getting anatomic and perfusion data with only one sequence we will possibly reduce sacnning time significantly. We started therefore a prospective study.

\section{1-P.13}

Category: Diagnostic Brain

Presentation Type : Poster Presentation

\section{Keywords :}

\section{Risk Factors and Characteristics of the Ulcerated Carotid Plaque}

Loraine Fisch ${ }^{1}$, Dana Niry ${ }^{2}$, Rolf Jager ${ }^{2}$, Martin Brown ${ }^{1}$

National Hospital for Neurology and Neurosurgery Neurology LondonUnited Kingdom ${ }^{1}$ National Hospital for Neurology and Neurosurgery Neuroradiology London-United Kingdom ${ }^{2}$

Introduction: Ulcers are considered as one of the high-risk components of the carotid plaque occurring in 14 to $68 \%$ of all stenosis. This study aims to determine cardiovascular risk factors and characteristics of symptomatic ulcerated carotid stenosis.

Method: We retrospectively selected 100 consecutive patients with a carotid stenosis visualized on both $\mathrm{CT}$ and MR angiography after a stroke or a TIA. A radiologist and a neurologist assessed all arteries on both imaging independently. We compared three plaque surface types (smooth, irregular and ulcer) in terms of risk factors, plaque length and degree of stenosis.
Results: 44, 31 and 25 patients were included in the smooth, irregular and ulcer groups respectively. Males were predominant in the ulcer group ( 23 vs 19 in the smooth group, $\mathrm{p}=0.003$ ). High blood pressure and cholesterol were predominant in the ulcer group compared to the smooth group $(80 \%$ vs $55 \%, \mathrm{p}=0.03 ; 69 \%$ vs $34 \%, \mathrm{p}=0.007$; respectively). Others cardiovascular risk factors and the degree of stenosis were equally distributed in groups. Average length of the plaque was $25 \mathrm{~mm}$ on CT and $23 \mathrm{~mm}$ on MR in the ulcerated group compared to $21 \mathrm{~mm}$ and $16 \mathrm{~mm}$ in the smooth group, respectively $(\mathrm{p}=0.018$ and $\mathrm{p}<0.001$ respectively).

Conclusion: Ulcerated plaques are found in male patients with high blood pressure and hypercholesterolemia. Development of ulcers is correlated with the length of the plaque but not with the degree of stenosis.

\section{1-P.14}

Category: Diagnostic Brain

Presentation Type : Poster Presentation

\section{Keywords :}

Does Systemic Lupus Erythematosis (SLE) cause chronic, progressive alteration of functional connectivity in intra-cerebral networks? A Resting state fMRI-study.

Jessika Nystedt ${ }_{-}^{1}$, Pia C Sundgren ${ }^{1}$, Andreas Jönsen ${ }^{2}$, Petra Nilsson ${ }^{3}$, Åsa Lilja ${ }^{4}$, Peter Mannfolk ${ }^{5}$, Olof Strandberg ${ }^{6}$

Division of Clinical Radiology Department of Clinical Sciences LundSweden ${ }^{1}$ Division of Rheumatology Department of Clinical Sciences Lund-Sweden ${ }^{2}$ Division of Neurology Department of Clinical Sciences Lund-Sweden ${ }^{3}$ Center for primary Health Care Department of Clinical Sciences Lund-Sweden ${ }^{4}$ Institution Clinical Imaging and Physiology Department of Clinical Sciences Lund-Sweden ${ }^{5}$ Clinical Memory Researh Unit Department of Clinical Sciences Lund-Sweden ${ }^{6}$

Purpose: To investigate if functional connectivity was altered in SLEpatients and if these alterations were more pronounced in SLE-patients with documented neuro-psychiatric symptoms (NPSLE).

Materials and Methods: 70 female SLE patients (age 20-54, mean 38,5) and 20 age and gender matched HC:s (age 25-55, mean 38,8) was included in this study.

Patients were further sub-categorized according to their level of Neuropsychiatric symptoms according to the ACR and Hanly-criterias.

All subjects underwent clinical examination and advanced MRI investigation, including resting state-fMRI.

A gradient-echo EPI pulse sequence was used (TR/TE $=1850 / 30 \mathrm{~ms}$, inplane resolution $=3 \times 3 \mathrm{~mm} 2$, slice thickness $=3 \mathrm{~mm}, 256$ volumes).

Preprocessing of fMRI data was performed using an FSL-based pipeline (normalization, physiological noise correction and motion artifact reduction) and resampled into $5 \times 5 \times 5 \mathrm{~mm} 3$ voxels. Connectivity analysis was performed by calculating pairwise correlation between all re-sampled voxel time courses. Group comparisons were made using permutation tests, controlling for multiple comparisons.

Results: In the non-NPSLE group, hyper-connectivity was observed inbetween the Ventral Attention Network (VAN), Dorsal Attention Network (DAN), Fronto-Parietal network (FPN) and the Sensory Motor Area (SMA). Hyper-connectivity was more pronounced in the NPSLEgroups.

Hypo-connectivity both within the default mode network (DMN) and in the salience network were identified in the NPSLE sub-groups in comparison to HCs.

Conclusion: Our findings are highly interesting and might indicate that SLE, in a progressive manner alters intracerebral connectivity in networks essential cognitive function as well as for consolidation of memories. These alterations might be compensatory or due to decreased network integrity. Further correlation-analysis will be preformed. 


\section{1-P.15}

Category: Diagnostic Brain

Presentation Type : Poster Presentation

Keywords : spontaneus intracerebral hemorrhage, basal ganglia, the natural course

The natural course of spontaneous intracerebral hemorrhage in basal ganglia - the analysis of a series of 39 patients

Aleksandra Milosevic $^{1}{ }_{-}$, Goran Tasic ${ }^{2}$, Mileusnic Valentina ${ }^{3}$, Katarina Kacar ${ }^{1}$

Special Hospital "Sveti Sava" Radiology Belgrade-Serbia ${ }^{1}$ Clinical Center of Serbia Neurosurgery Belgrade-Serbia ${ }^{2}$ Special Hospital "Sveti Sava" Neurology Belgrade-Serbia ${ }^{3}$

Introduction: Spontaneous intracerebral hemorrhage (SICH) represent acute stroke and make about $10-15 \%$ of all strokes and are associated with high mortality and morbidity. SICH rarely occur in the age group below 45 years. Material and Methods: The study included 39 patients treated in a Special Hospital for CVD "Sveti Sava" in the period from October 2010 to January 2012. Including criteria was a diagnosis of SICH in patients in whom other pathological substrate that could cause SICH is excluded. In all patients it was determined: gender, age, localization of SICH in the basal ganglia on the basis of MDCT and presence of intraventricular hemorrhage, outcome (recovery or lethal). Head MDCT (16 slices, bright speed, GE) was performed on admission and follow- up.

Results: There was 25 male and 14 female patients, a range from 54 to 95 years. The most frequent localization was: putamen (23), globus pallidus (20), internal capsule (19), nc lentiformis (9). Acute hydrocephalus was complication in 26 patients $(66.6 \%)$. In this group 20 patients $(77.3 \%)$ had lethal outcome. Lethal outcome in first $12 \mathrm{~h}$ had 9 patients $(34.4 \%)$, while 17 patients $(55.4 \%)$ had lethal outcome in first $24 \mathrm{~h} .13$ patients $(56.5 \%)$ sent to rehabilitation.

Conclusion: Predisposing factors for mortality within one year in patients with $\mathrm{SICH}$ in the basal ganglia of the brain are: males older than 60 years, unregulated hypertension and blood in the ventricular system.

\section{1-P.16}

Category: Diagnostic Brain

Presentation Type : Poster Presentation

Keywords : AIDS. brain. network. DIffusion. tensor. MRI.

Local Network Impairment in Asymptomatic Young AIDS Patients with Normal Brain MRI: Diffusion Tensor Imaging Connectome Analysis

Snag-Hoon Cha ${ }^{1}$, Chi-Hoon $\mathrm{Choi}^{2}$, Kyung Sik Yi ${ }^{2}$

College of Medicine, Chungbuk National University Radiology Cheongju-Korea, South ${ }^{1}$ Chungbuk National University Hostpital Radiology Cheong-ju-Korea, South ${ }^{2}$

Objectives: To know local network impairment in asymptomatic young AIDS-patients with normal MRI with diffusion tensor imaging connectome analysis.

Methods: We analyzed diffusion tensor imaging of asymptomatic young male AIDS patients $(\mathrm{n}=15,31$ years +6.02$)$ and compared with agematched male controls $(34$ year +8.02$)$. Diffusion tensor whole-brain fiber tractograms (1.5T-MRI, by FACT, 15 direction, $2 \times 2 \times 4 \mathrm{~mm}$ ) were inversely transformed into T1 space (3D-MPGR) and fiber tracts and AAL-based parcellated regions ( 78 areas, 39 regions in each hemisphere) were located in the same space and to define the nodes of a brain graph. A mean fractional anisotropy value multiplying fiber number along all the fibers connecting a pair of regions was used to weight the edge. Finally, weighted WM networks represented by symmetric $78 * 78$ matrices were constructed for each individual. Graph theoretical analyses were performed on weighted connectivity networks using the Brain Connectivity Toolbox (www.brain-connectivity-toolbox.net).

Results: Young male AIDS patients showed significant multifocal areas of low local efficiency and strength $(p<0.05)$ without cortical thickness differences.

Conclusions: Young male AIDS patients showed altered brain connectivity and intergrity. Their local netwrok impaired in spite of neurologically asymptomatic patients with normal brain MRI.

\section{1-P.17}

Category: Diagnostic Brain

Presentation Type : Poster Presentation

Keywords : Ferumoxytol, Artifacts, Iron

Magnetic Resonance Imaging Findings of the Brain Related to Intravenous Administration of Ferumoxytol.

Norbert Campeau ${ }_{-}$, Gary Miller ${ }^{1}$

Mayo Clinic Radiology Rochester-United States ${ }^{1}$

Purpose: The purpose of this work was to describe potentially confounding brain MR imaging (MRI) findings which can occur following intravenous ferumoxytol therapy for treatment of iron deficiency anemia, the administration of which was unknown to the radiologist at the time of interpretation.

Methods: MRI brain examinations from four patients who were imaged within a few days of having received intravenous ferumoxytol for treatment of iron deficiency presented with unusual imaging findings, which after further investigation were determined to be related to the effects of ferumoxytol. Results: Exaggerated loss of signal intensity within the vasculature on T2* gradient echo imaging and susceptibility weighted images can mimic the appearance of superficial siderosis or diffuse thrombosis. On T1 weighted gradient echo imaging, ferumoxytol behaves like a positive contrast agent, resulting in high signal in all vessels. Maximum intensity projection of 3D $\mathrm{T} 1$ gradient echo acquisitions can provide angiographic information similar to that obtained with an MRV. Time-of-flight MRA suffers from venous contamination, similar to that noted when obtained following IV gadolinium. On spin echo T1 imaging, ferumoxytol masks the expected enhancement from IV gadolinium, and resulted in a second administration of gadolinium in one patient, as the lack of expected enhancement was believed to be on the basis of misadministration possibly related to IV infiltration. Conclusion: Awareness of the MRI findings and artifacts related to intravenous ferumoxytol is important to avoid additional imaging or unnecessary medical intervention.

\section{1-P.18}

Category: Diagnostic Brain

Presentation Type : Poster Presentation

Keywords : osteogenesis imperfecta; giant serpentine aneurysm, spontaneous thrombosis

Spontaneously thrombosed giant serpentine aneurysm in a patient with osteogenesis imperfecta

Alja Longo $_{2}{ }_{-}$, Tina Vipotnik Vesnaver ${ }^{2}$

University Medical Centre Ljubljana Clinical Institute of Radiology Ljubljana-Slovenia ${ }^{1}$ University Medical Centre Clinical Institute of Radiology Ljubljana-Slovenia ${ }^{2}$ 
Background: Osteogenesis imperfecta (OI) is a group of hereditary connective tissue disorders causing increased osseous fragility. Its extraskeletal manifestations can also involve the cardiovascular system. Cerebral aneurysms have been reported to be associated with OI.

Giant serpentine aneurysms form a subgroup of large intracranial aneurysms with characteristic imaging features.

We report a case of a giant serpentine aneurysm in a patient with OI. Case Presentation: 40-year-old male with known OI was admitted to our hospital with acute neurological symptoms. Initial CT and CTA showed an expansive lesion with surrounding edema in the left frontotemporal region. There was a tortuous tubular structure in the lateral portion of the lesion that partially enhanced after contrast administration. Control CTA five days later showed no enhancement. MRI and DSA confirmed the presence of a giant serpentine aneurysm of the left MCA, which ruptured and later thrombosed spontaneously. On follow-up imaging, the lesion progressively decreased in size.

Discussion: A prominent pathologic feature of cerebral artery aneurysms is reduced collagen content. Defective collagen in OI could therefore be directly associated with increased vascular weakness. However, compared to some other connective tissue diseases, predilection for aneurysms in OI is low. To our knowledge, ours is the first case of a giant serpentine aneurysm in a patient with OI.

\section{1-P.19 \\ Category: Diagnostic Brain \\ Presentation Type : Poster Presentation}

\section{Keywords :}

\section{A case of neurosarcoidosis}

$\frac{\text { Annapaola Bocchio }}{\text { Teodoro }^{1}}{ }^{1}$, Davide Machado ${ }^{1}$, Rosset Massimo ${ }^{1}$, Meloni Regional Hospital Valle d Radiology aosta-Italy ${ }^{1}$

Sarcoidosis is an immune-mediated systemic disease, characterized by non-caseating epitheliod granulomas, located, in the $90 \%$ of patients, in the lungs or related lymph-nodes. However,lesions can affect any organ. Neurosarcoidosis may develop in up to $10 \%$ of the patients, either in addition to systemic manifestations or isolated.

We present the case of a 24 years-old male, occuring for headache and seizures. Neurological examination at emergency: normal, except for right VII c.n deficit.CT: large hypodensity of left deep white matter, sparing the cortex..MRI:multiple infra and supratentorial contrastenhancing small intraparenchymal nodules, with large perifocal aedema. Nodular thickening of the leptomeninges and ventricular ependyma. Chest X-Ray: multiple small pulmonary masses; Contrast Enhanced Chest-Abdomen CT: multiple, bylateral small pulmonary nodules; enlarged hilar and mediastinal lymph-nodes, whith increased metabolic activity on FDG-PET-CT. Abdomen was normal.Mediastinoscopy: lymphnodes biopsies. Histology: epithelial granulomatosis, consistent with sarcoidosis. Patient underwent steroid therapy. MRI follow-up, after, 3, 8 and 12 months: progressive reduction of the pathological parenchymal and meningeal nodules.

Conclusions: Sarcoidosis can be insidious for radiologists, showing different imaging features, often non-specific. The diagnosis requires compatible clinical findings, histologic demonstration of noncaseating granulomas and exclusion of other diseases. MRI is the most sensitive test for Neurosarcoidosis, with a wide variety of radiological features. The most common is leptomeningeal involvement, usually seen as thickening and diffuse or nodular enhancement, often in addition with solitary or multiple enhancing intraparenchymal masses, mimicking other neurological conditions (multiple sclerosis, acute disseminated encephalomyelitis, infections and neoplasms). Awareness of these features orients radiologists in the diagnosis.
1-P.20

Category: Diagnostic Brain

Presentation Type : Poster Presentation

Keywords : DTI. head Injury

DTI: Assessment of diffuse axonal injury in moderate and severe traumatic brain injury

Amaya Hilario ${ }^{1}$, Ana Maria Castaño ${ }^{2}$, Elena Salvador ${ }^{1}$, Laura Koren ${ }^{1}$, $\overline{\text { Patricia Martin }}^{\top}$, Alfonso Lagares ${ }^{2}$, Ana Ramos ${ }^{1}$

University Hospital 12 de Octubre Radiology Madrid-Spain ${ }^{1}$ University Hospital 12 de Octubre Neurosurgery Madrid-Spain ${ }^{2}$

Introduction and Objectives: Our objective is to correlate FA values at white matter (WM) tracts with neurological outcome in patients with moderate and severe TBI.

Methods and Material: Eighty patients with TBI $(\mathrm{GCS}<13)$ were admitted to our hospital (January 2008-December 2012). DTI was performed using 25 diffusion-encoding directions. To obtain FA values we placed ROIs in the corpus callosum, internal capsule (anterior and posterior limbs ALIC-PLIC), external capsule (EC), forceps major, forceps minor, cingulum, superior longitudinal fasciculus (SLF), inferior fronto-occipital fasciculus, inferior longitudinal fasciculus, corona radiate (anterior aCR, posterior and superior) and cerebral peduncles (CP).

We calculated Pearson correlation coefficient between FA values and patient outcome at hospital discharge and after 6-month after trauma (extended GOS). We also evaluated which portion of the corpus callosum best discriminate patient outcome.

Results: We included 30 moderate and 50 severe head injuries (21 females and 59 males; mean age 35 years). Low FA values showed a significant correlation with poor outcome in all portions of the corpus callosum, right sCR, left SLF, left ALIC, both PLIC, both EC and CP. Pearson correlation coefficient showed a range of FA values between 0.283 and 0.514 . The genu of the corpus callosum was the WM tract that best discriminated patient outcome.

Conclusions: Low FA values were associated in moderate and severe TBI with poor outcome at hospital.Low FA values were associated in moderate and severe TBI with poor outcome at hospital discharge and 6 months post-injury. The genu of the corpus callosum showed the highest correlation with outcome.

\section{1-P.21}

Category: Diagnostic Brain

Presentation Type : Poster Presentation

\section{Keywords : AMYLOIDOSIS}

\section{How to suspect Cerebral Amyloidosis}

Ana Ramos ${ }^{1}$, Amaya Hilario ${ }^{1}$, Elena Salvador ${ }^{1}$, Laura Koren ${ }^{1}$, Patricia Martin $^{1}$, Federico Ballenilla ${ }^{1}$

Hospital 12 de Octubre Radiology Madrid-Spain ${ }^{1}$

Purpose: Amyloidosis is a generic term that includes a group of diseases with one common feature: the progressive deposition of $\beta$ - amyloid peptide, an extracellular protein, insoluble and resistant to proteolysis.

The amyloid deposition may take many forms in central nervous system: the cerebral amyloid angiopathy (CAA), the amyloid angiitis variant and the pseudotumoral form or amyloidoma.

Approach/Methods: We review radiological findings of the various forms of cerebral amyloidosis admitted to our hospital during the past 5 years. 
Findings and Discussion: CAA is characterized by progressive deposition of amyloid- $\beta$ peptide in the walls of small- to medium-sized arteries, arterioles, and capillaries of the cerebral cortex and overlying leptomeninges. Intracranial hemorrhage is the most common pathological manifestation. It usually occurs sporadically but sometimes is associated with Alzheimer's disease, Kuru or Creutzfeldt-Jakob.

In amyloid angiitis, patients develop vascular and perivascular inflammatory changes that radiologically manifest as vasogenic edema, leptomeningeal enhancement and microbleedings. The differential diagnosis should be done with primary CNS vasculitis, posterior reversible encephalopathy and progressive multifocal leukoencephalopathy.

The pseudotumoral variant or amyloidomas are focal amyloid deposits that can become large with little or no mass effect. They behave like slowgrowing tumors and differential diagnosis must be done indeed with brain neoplasms.

Summary and Conclusion: The term amyloidosis refers to a group of diseases characterized by extracellular deposition of $\beta$-amyloid peptide. The cerebral amyloid angiopathy, the amyloid angiitis and the pseudotumoral variant or amyloidoma are the diverse forms whose radiological manifestations are different from each other and the radiologist must know.

\section{1-P.22}

Category: Diagnostic Brain

Presentation Type : Poster Presentation

Keywords : Infections.immunocompromised

\section{How to aproach cns infections in the compromised host}

Laura Koren ${ }^{1}$, Elena Salvador ${ }^{1}$, Amaya Hilario ${ }^{1}$, Patricia Martin ${ }^{1}$, Federico Ballenilla ${ }^{1}$, Jose Maria Millan ${ }^{1}$, Ana Ramos ${ }^{1}$

Hospital 12 de Octubre Radiology Madrid-Spain ${ }^{1}$

Purpose: Infections of the central nervous system (CNS) are a common condition with a high morbidity in the immunocompromised host.

A compromised immune system not only predisposes the host to a higher incidence of infections but considerably broadens the spectrum of responsible pathogens. Besides the common pathogens in the general population, there are others that are almost exclusive to patients with impaired immunity as Toxoplasma, Cryptococcus or JC virus. Initial symptoms may be nonspecific as these patients may have a reduced response to infection and therefore less clinical and analytical repercussion. Complementary tests such CT and MRI play therefore a key role in the diagnosis. The aim of the paper is to review the radiological findings in opportunistic infections of the CNS and determine a possible etiologic classification according to the main finding.

Approach and Methods: We review the imaging tests of immunocompromised patients with CNS infections admitted to our hospital during the past 10 years.

Findings/Discussion: We classify the imaging findings into five categories: mass effect lesions, diffuse involvement of the white matter, meningeal involvement, ventricular involvement and focal parenchymal involvement (encephalitis).

Even though different microorganisms can exhibit a wide overlap of imaging features, there are certain patterns that can point to a specific pathogen so depending on the predominant finding we perform a possible etiologic classification.

Summary and Conclusion: CNS opportunistic infections are a common condition with high morbidity and mortality rates. Clinical manifestations in the immunosuppressed patient are often nonspecific so it is important that the radiologist is familiar with the imaging findings that allow an apropiate approach.
1-P.23

Category: Diagnostic Brain

Presentation Type : Poster Presentation

Keywords : MRA. MR Angiography

Techniques in Mr Angiography of the Brain: What To Do With Them

Patricia Martin ${ }^{1}$, Amaya Hilario ${ }^{1}$, Elena Salvador ${ }^{1}$, Laura Koren ${ }^{1}$, Federico Ballenilla ${ }^{1}$, Ana Ramos ${ }^{1}$

Hospital 12 de Octubre Radiology Madrid-Spain ${ }^{1}$

Purpose:

- To know MRI angiography techniques and its basic physical principles.

- To describe the advantages, limitations and potential pitfalls of each technique.

- To develop a practical diagnostic algorithm depending on the clinical context.

Approach: MRI angiography is one of the most commonly used noninvasive techniques for imaging of the cerebrovascular system.

Discussion: Neurovascular studies by MRI can be performed by different techniques:

1. Phase contrast sequences, without contrast. It uses magnetization differences between flowing blood and stationary tissues. Thus, the vascular anatomy can be assesses, and the speed and direction of the blood flow can be quantitatively determined. Three-dimensional (3D) acquisition is used for depicting venous thrombosis and as a non-contrast technique.

2. 3D time-of-flight (TOF) sequences, with and/or without contrast. They derive contrast between flowing blood and stationary tissues by manipulating the magnitude of the magnetization. $3 \mathrm{D}$ TOF sequence is commonly used in the evaluation of the circle of Willis. It presents limitations in peripheral small vessels, giant or thrombosed aneurysm and in those treated with stent.

3. Contrast-enhanced MR angiography, as either dynamic (time-resolved) or fixed single-phase (arterial or venous) imaging. It is not affected by complex or slow flow, provides high quality images with fewer artifacts and large fields of view can be imaged in a short acquisition time.

Conclusion: MR angiographic techniques are routinely used in neurovascular imaging. A better understanding of its physical principles, advantages, limitations and pitfalls will allow obtaining diagnostically useful images in each clinical situation.

\section{1-P.24}

Category: Diagnostic Brain

Presentation Type : Poster Presentation

Keywords : meningioma, brain metastasis, ferumoxytol, USPIO, GBCA, MRI contrast agent

Late ferumoxytol enhancement aids the differentiation between meningiomas and metastases.

Laszlo Szidonya ${ }^{1}$, Csanad Varallyay ${ }^{2}$, Bronwyn Hamilton ${ }^{2}$, Prakash Ambady ${ }^{1}$, Edward Neuwelt ${ }^{1}$ 
Oregon Health and Science University Dept. of Neurology, Blood-Brain Barrier and Neuro-Oncology Program Portland-United States ${ }^{1}$ Oregon Health and Science University Dept. of Diagnostic Radiology Portland-United States ${ }^{2}$

Introduction: Initial studies by Varallyay, $\mathrm{P}$ et al. showed that meningiomas do not enhance with the USPIO ferumoxtran-10 in contrast to gadolinium-based contrast agents (GBCA) presumably due to the difference in the size of the two contrast agents. Hamilton et al. extended these observations by showing that utilizing the USPIO as a supplement to GBCA could differentiate dural-based GBCA-enhancing masses, correlating the difference in permeability with the expression of connexin-43. In the current report the difference in permeability of brain metastases vs meningiomas was further documented by comparing normalized contrast enhancement with ferumoxytol vs GBCA.

Methods: Normalized contrast enhancement was retrospectively measured by two neuroradiologists in 20 patients ( 10 with meningiomas, 10 with brain metastases) receiving ferumoxytol in 3 IRB-approved protocols. Standard normalized contrast enhancement values were obtained from the immediate post-GBCA and the 24 hour post-ferumoxytol scans. Results: For meningiomas the average of the 24 hour mean values of normalized ferumoxytol enhancement were between 1.16-1.17 for the two readers (significantly different from the 1.76-1.86 obtained with GBCA). In the metastatic tumors, which also avidly enhanced with GBCA (1.791.96), the average normalized values of late ferumoxytol enhancement were between 1.59-1.62. These were statistically significantly different from the average ferumoxytol enhancement of meningiomas $(\mathrm{p}<0.05)$.

Conclusion: Consistent with 2 previous reports, in the current study ferumoxytol demonstrated a stark difference in normalized contrast enhancement vs GBCA strongly suggesting that meningiomas can be reliably differentiated from brain metastases by using both contrast agents even when by standard neuroradiological appearance the lesions may appear virtually the same.

\section{1-P.25}

Category: Diagnostic Brain

Presentation Type : Poster Presentation

Keywords : head injury, peritentorial, subdural hematoma, Glasgow coma scale

Traumatic peritentorial subdural hematomas - meaning and significance of CT findings

Jelena Kostic ${ }^{1}$, Irena Kostic ${ }^{2}$, Ana Stankovic ${ }^{1}$, Kristina Davidovic ${ }^{1}$, Faris Crnovrsanin ${ }^{1}$, Danilo Stankovic ${ }^{1}$, Ruzica Maksimovic ${ }^{3}$

Clinical Center of Serbia Emergency department Belgrade-Serbia

University of Kragujevac Department for Pathophisiology KragujevacSerbia ${ }^{2}$ Clinical Center of Serbia Center for radiology and MRI Belgrade-Serbia ${ }^{3}$

Aim: This sudy aimed to investigate clinicoradiological features in patients with traumatic peritentorial subdural hematomas (SDH).

Material and Methods: We retrospectively reviewed the clinical and radiological findings, management criteria and outcomes in 120 patients with peritentorial SDH treated at the Emergency Department Clinical Center of Serbia, Belgrade. The outcomes were classified as good recovery or moderate disability or severe disability/death according to the Glasgow coma scale (GCS)

Results: Our study group counted 120 patients, 78 were male and 42 were female. The patients' ages ranged from 4-90 years (mean age 60.9 years). Coagulopathies were observed in 23 patients. Of all patients, $80,5 \%$ had GCS $13-15$ that among those $45 \%$ had GCS 15 . Furthermore, $14 \%$ had GCS ranged 9-12 and 9\% had GCS $\leq 8$. Of all patients with abnormal CT findings, $45 \%$ underwent surgery that $60 \%$ categorized in mild head injury group, $18 \%$ categorized in moderate head injury group and $22 \%$ categorized in severe head injury group. Other patients were treated conservatively. The presence of coagulopathy $(\mathrm{p}=0.024)$ and presence of convexity SDH $(\mathrm{p}=0.008)$ correlated with the outcome.

Conclusion: The patients with traumatic peritentorial SDHs were predominantly male and relatively elderly, and had a high incidence of coagulopathy, associated intracranial lesions (especially falks SDH), a high rate of impact in the occipital or frontal regions. The use of GCS score for assessing the level of injury may not be sufficient, so the combination of this scoring system and other applicable scoring systems may be more applicable

\section{1-P.26}

Category: Diagnostic Brain

Presentation Type : Poster Presentation

Keywords : 3D-printing, cerebral aneurysm, simulation

Assessment the utility of three-dimensional printed cerebral aneurysm model in neck clipping operation

Toshi Abe ${ }^{1}$, Ayako Yoshida ${ }^{1}$, Shuji Nagata ${ }^{1}$, Yusuke Uchiyama ${ }^{1}$, Takachika Äoki ${ }^{2}$, Reiji Katayama ${ }^{3}$, Norimitsu Tanaka ${ }^{1}$

Kurume University School of Medicine Department of Radiology Kurume-Japan ${ }^{1}$ Kurume University School of Medicine Department of Neurosurgery Kurume-Japan ${ }^{2}$ Kurume University Hospital Center for Diagnostic Imaging Kurume-Japan ${ }^{3}$

Purpose: To assess the utility of three-dimensional printed cerebral aneurysm (3DP-AN) models, which we have newly proposed for neurosurgical aneurysmal neck clipping planning.

Materials and Methods: 16 aneurysms (9 MCA, 4 AcomA, 2 IC-PC, and 1 AchA) of 14 patients (5 men and 9 women; age range 35-75 years) in whom preoperative 3DP-AN models were printed. Two patterns of 3DP-AN models were printed from same data set. Luminal model:a solid model as vessel lumen. Outward model:a solid model, which surround vessel lumen with $0.4 \mathrm{~mm}$ thick vessel wall layers as outer form of the vessel.

Results: In this study, the surgeons in training showed a greater tendency to change the clip, based on the 3DP-AN models, compared with that showed by the operators. The outward models considering the thickness of the vessel wall are close to the surgical findings than the luminal models.

Conclusion: 3DP-AN model may be effective for preoperative simulation, especially for the young surgeons.However, further improvement of reality is required. For example, the soft model which can apply a clip or the model with surrounding anatomy.

\section{1-P.27}

Category: Diagnostic Brain

Presentation Type : Poster Presentation

\section{Keywords :}

Optimized visualization of early cerebral infarcts after endovascular stroke therapy with dual-energy computed tomography

$\underline{\text { Tanja Djurdjevic }}{ }^{1}$, Thomas Schiestl ${ }^{1}$, Florian Dazinger ${ }^{1}$, Bernhard Glodny $^{2}$, Rafael Rehwald ${ }^{2}$, Michael Knoflach ${ }^{3}$, Elke Ruth Gizewski ${ }^{1}$, Astrid Ellen Grams ${ }^{1}$

Medical University of Innsbruck Department of Neuroradiology Innsbruck-Austria ${ }^{1}$ Medical University of Innsbruck Department of Radiology Innsbruck-Austria ${ }^{2}$ Medical University of Innsbruck Department of Neurology Innsbruck-Austria ${ }^{3}$ 
Background and Purpose: After endovascular stroke therapy (EST) the depiction of early cerebral infarcts may be limited with conventional CT, due to hyperdense masking from hemorrhage or BBB disruption. The aim of this study was to investigate if post-processed dual-energy CT (DECT) series allow a better identification of early infarcts than brain window series.

Materials and Methods: In this study 47 patients (21 women and 26 men), who received a DECT immediately after EST, and who displayed cerebral infarcts on follow-up imaging, were included. A brain window (BW), a virtual non-contrast (VNC) and a modified VNC series, focusing on densities of infarct and cerebral cortex, named "edema map" (EM), were evaluated. ROI measurements were performed on all series in one or more infarct areas per patient and in the cerebral cortex, from which cortexinfarct differences (CID) were calculated. In addition infarct identifiability, severity of artifacts and disturbance from hyperdense materials were evaluated with subjective rating scales.

Results: Significant CIDs were found with the VNC and the EM series $(p<0.0001)$, with the highest values in the EM series $(52.1+/-77.7)$. Best infarct identification featured the EM series, without any disturbance from hyperdense materials.

Conclusion: With EM a higher contrast and better identifiability of early infarcts after EST can be achieved, in comparison to VNC or BW series, without disturbance from hemorrhage or BBB disruptions. This could feature the opportunity to modify early therapy decisions, such as craniotomy.

1-P.28

Category: Diagnostic Brain

Presentation Type : Poster Presentation

Keywords : Internal carotid artery, Carotid plaque, MRI

Hemorrhagic Plaque of Carotid Artery on MRI Predicts Recurrent Ipsilateral Ischemia and Progression of Stenosis: A Single Center Study

$\underline{\text { See Sung Choi }}{ }^{1}$, Se Jeong Jeon ${ }^{1}$, Hye Ji Ryu ${ }^{1}$

Wonkwang University Hospital Radiology Iksan-Korea, South ${ }^{1}$

Purpose: We assessed the MR-defined intraplaque hemorrhage (IPH) of carotid artery to predict recurrent ipsilateral cerebral ischemic events and progression of stenosis degree.

Material and Methods: 74 patients underwent plaque imaging using 3D inversion-recovery-based T1WI (MPRAGE). Among these, 31 carotid arteries in 27 patients had stenosis. IPH was defined if plaque signal intensity on MPRAGE of $>150 \%$ that of adjacent muscle. We assessed ipsilateral ischemic event rates and change of the plaque volume (progression of stenosis) between arteries with and those without IPH.

Results: Of the 31 carotid arteries included 9 (29\%) had IPH and 22 (71\%) had non-hemorrhagic plaque. The rate of recurrent acute stroke is $30 \%(3 / 9)$ in carotid arteries with IPH and $13 \%(3 / 22)$ without IPH but there is no significant difference $(\mathrm{p}=0.32)$. Change of plaque volume was more frequently seen in carotid arteries with IPH than without IPH (44\% vs $0 \%, \mathrm{p}=0.004)$. In two cases $(50 \%)$ of plaque progression, recurrent stroke occurred with the increase of plaque volume.

Conclusions: IPH was associated with progression of stenosis and tends to increase rate of recurrent ischemic events. If the symptom that is suspected ischemic event occurs in the patients with IPH during the treatment, it can be the sign of new ischemic cerebral infarction with the increased plaque volume. Therefore follow-up MR imaging and aggressive treatment are thought to be necessary for the patients who had carotid stenosis with IPH, even if stenosis of carotid artery is mild or asymptomatic.
1-P.29

Category: Diagnostic Brain

Presentation Type : Poster Presentation

Keywords : Mild cognitive impairment, FSL, subcortical gray matter structures

Volumetric Change of Subcortical Gray Matter Structures in Mild Cognitive Impairment: A Longitudinal MRI Study

Berrin Cavusoglu ${ }^{1}$, Emel Ada $^{2}$, Derya Durusu Emek Savas ${ }^{3}$, Gorsev Yener ${ }^{4}$

Dokuz Eylul University Neuroscience IZMMiR-Turkey ${ }^{1}$ Dokuz Eylul University Radiology İZMIR-Turkey ${ }^{2}$ Dokuz Eylul University Psychology İZMIR-Turkey ${ }^{3}$ Dokuz Eylul University Neurology IZMIR-Turkey ${ }^{4}$

Purpose: Patients with amnestic mild cognitive impairment (MCI) have an increased risk of developing Alzheimer's disease (AD) and represent a predementia stage of $\mathrm{AD}$. We aimed to evaluate longitudinal changes in the volume of subcortical deep gray matter structures in patients with amnestic MCI, using FSL software package (FMRIB Software Library, http://www.fmrib.ox.ac.uk/fsl/ fslwiki/).

Methods: The study included 22 patients with amnestic MCI. All participants were scanned twice at one year follow-up with axial TSE T2 weighted and sagittal 3 dimensional GRE T1 weighted magnetic resonance imaging. Volumetric data for regional volumes of subcortical structures (thalamus, caudate nucleus, putamen, globus pallidus, nucleus accumbens, hippocampus, amygdala) were obtained by using the algorithm FIRST (FMRIBs integrated registration and segmentation tool) which is provided in FSL. Volumetric comparisons were performed by using wilcoxon test $(\mathrm{p}<0,05)$.

Results: No significant differences in subcortical volumes were found, except hippocampus. The longitudinal volumetric measurements of MCI patients showed that significant reduced rate of hippocampal atrophy about $4,5 \%$ over one year period $(\mathrm{p}=0,01)$.

Conclusion: Assessment of volume loss of hippocampus over time in MCI have the potential to help in monitoring the course of disease in patients with increased risk of progression from $\mathrm{MCI}$ to $\mathrm{AD}$.

This study was supported by TUBITAK 112S459 grant.

1-P.30

Category: Diagnostic Brain

Presentation Type : Poster Presentation

Keywords :

Prognostic Value of mr Spectroscopy in High Grade Astrocytomas. Validation of Previous Results.

Albert Pons ${ }^{1}$, Juan J Sánchez- Fernández ${ }^{1}$, Àngels Camins ${ }^{1}$, Margarida Julià-Sapé $^{2}$, Miguel Gil ${ }^{3}$, Carles Arús ${ }^{4}$, Carles Aguilera ${ }^{1}$, Carles Majós ${ }^{1}$

IDI Centre Bellvitge. HU de Bellvitge. Radiology. L'Hospitalet de Llobregat-Spain ${ }^{1}$ Universitat Autònoma de Barcelona. Centro de Investigación en Red en Bioingeniería, Biomateriales y Nanomedicina (CIBER-BBN). Unitat de Bioquímica de Biociències. Cerdanyola del Vallès.-Spain ${ }^{2}$ ICO 1'Hospitalet. HU de Bellvitge. Medical Oncology. L-Spain ${ }^{3}$ Universitat Autònoma de Barcelona. Unitat de Bioquímica de Biociències. Centro de Investigación en Red en Bioingeniería, Biomateriales y Nanomedicina (CIBER-BBN). Cerdanyola del Vallès.Spain $^{4}$

Background and Purpose: Prognosis for patients with High-Grade Astrocytomas (HGA) is globally poor. There is a large range of 
survival that can only be partially explained by histologic grade and clinical aspects. Predictive radiologic factors have been studied extensively. Previous studies demonstrated 1H-MRS (proton MR spectroscopy) value in predicting survival. This retrospective study aims to validate those results in a new independent data-set of patients.

Material and Methods: Pre-treatment H-MRS in 212 patients with HGA produced 207 spectra at short TE (STE, 30ms) and 201 at long TE (LTE, $136 \mathrm{~ms})$.

Patients were dichotomized according to the normalized intensities at the points that showed the highest prognostic value in a previous study: $0.98 \mathrm{ppm}, 3.67 \mathrm{ppm}$ and the ratio $3.67 / 0.98 \mathrm{ppm}$ at STE (cut-off values, $0.1024,0.0473$, and 0.5510 , respectively); and $0.98 \mathrm{ppm}$ and $1.25 \mathrm{ppm}$ at LTE (cut-off, 0.0267, 0.0098). Survival was analyzed with the Kaplan-Meier method.

In a second step, average spectra were constructed for patients dichotomized according to survival better or worse than the median (273 days), and differences between groups were evaluated.

Results: Significant differences in patient survival were confirmed at: $3.67 \mathrm{ppm}(\mathrm{P}=0.005)$ and ratio $3.67 / 0.98 \mathrm{ppm}(\mathrm{P}=0.004)$ at $\mathrm{STE}$; and $0.98 \mathrm{ppm}(\mathrm{P}=0.001)$ and $1.25 \mathrm{ppm}(\mathrm{P}=0.001)$ at LTE.

Average spectra showed relevant differences around 3.6-3.9 ppm at STE, and 1.2-1.4 ppm at LTE.

Conclusions: Previous results about $1 \mathrm{H}-\mathrm{MRS}$ prognostic value in HGA patients are reproducible.

We hypothesize that our results could translate specific cellular events that should be evaluated in future studies.

\section{1-P.31 \\ Category: Diagnostic Brain \\ Presentation Type : Poster Presentation}

Keywords : shwannomas, meningeomas, ependymomas, neurofibromatosis type 2

\section{Neurofibromatosis type 2 - typical presentation, Case report}

$\underline{\text { Radivoje Trpcevic }}^{1}$, Katarina Kacar ${ }^{1}$, Jovanka Pisarevic-Neskovic ${ }^{1}$

Special hospital for cerebrovascular diseases "Sveti Sava" Radiology Department Belgrade-Serbia ${ }^{1}$

Neurofibromatosis type $2(\mathrm{NF} 2)$ is a rare autosomal dominant neurocutaneous disorder (phakomatosis) manifesting as development of multiple CNS tumours. Patients with this disease have intracranial schwannomas (mostly vestibular schwannomas); intracranial and spinal meningiomas and intraspinal-intramedullary ependymomas.

We present 23-year-old man with a worsening bilateral hearing loss and no other neurological deficits.

On addmition MRI (GE Signa 1,5T) of the head and spine was performed and showed bilateral CN VIII schwannomas, multiple intracranial meningeomas as well as spinal ependymomas at the level of C2-C4, neurofibromas C6-C7, Th2-Th3, Th4 and L2. Positive familiar anamnesis.

Of patients with NF2, 50\% have an affected parent (autosomal dominant inheritance) and 50\%having a de novo mutation. Mutation of the NF2 gene on the long arm of chromosome 22 is responsible for the predisposition for tumour formation.

Neurofibromas are rarely found in NF2, which has led some to propose that the disease be termed schwannomatosis or MISME syndrome, the acronym for the following terms: Multiple inherited schwannomas Meningiomas Ependymomas

The modified criteria for definite diagnosis of NF2 are as follows:
1.Bilateral CN VIII schwannomas on MRI or CT scan

2.First-degree relative with NF2 and either unilateral early-onset CN VIII schwannoma (age $<30 \mathrm{y})$

CN schwannoma, including a unilateral CN VIII schwannoma or meningioma in a younger patient,suggests the possibility of NF2. Older patients with meningiomas or a unilateral CN VIII schwannoma should not undergo an expensive workup for NF2 because the disease usually presents before the age of 40 years. Patients older than 60 years with bilateral internal auditory canal masses are unlikely to have NF2 if it has not been diagnosed.

\section{1-P.32}

Category: Diagnostic Brain

Presentation Type : Poster Presentation

Keywords : Persistent trigemial artery, CT angiography, carotidvertebrobasilar anastomoses

A rare congenital carotid-vertebrobasilar anastomoses: Persistent trigeminal artery - Case report

Jelena Trickovic ${ }^{1}$, Katarina Kacar ${ }^{1}$, Radivoje Trpcevic ${ }^{1}$, Biljana Brkic-Georgievski ${ }^{1}$

Special hospital for cerebrovascular diseases Radiology Department Belgrade-Serbia ${ }^{1}$

Persistent primitive trigeminal artery (PPTA) is one of the persistent carotidvertebrobasilar anastomoses. It is present in $0.1-0.76 \%$ of cerebral angiograms and is usually unilateral. We present the $\mathrm{CT}$ angiograms of a patient who presented with a headache and was detected to have this rare anomaly. A 47-year-old woman with diffuse headache and history of arterial hypertension was observed in our ER with no evidence of neurological deficits. On admission we performed head CT and CT angiography (16 slices, GE). CT imaging of the brain showed no abnormalities, while the CT angiogram showed a persistent trigeminal artery on the left side with fetal origin of left ACP (Saltzman type II) which was an incidental finding. As another vascular variant a hypoplastic A1 segment of the right ACA was found. All variants found were incidental and asymptomatic. PPTA is a rare remnant of the embryonic circulatory system that unites the internal carotid and vertebrobasilar systems.

On the basis of angiographic characteristics, Saltzman classified PPTA into type I with absent ipsilateral posterior communicating artery and type II with fetal origin of ipsilateral posterior cerebral artery and absent P1 segment. Occasionally, the anastomosis represents a combination of these two variants. The clinical relevance of a persistent trigeminal artery is debatable as most cases are discovered incidentally, as it was the case with our patient. Although persistent trigeminal artery (PTA) is uncommonly identified, knowledge of this structure is essential for clinicians who interpret cranial imaging, perform invasive studies of the cerebral vasculature and operate this region.

1-P.33

Category: Diagnostic Brain

Presentation Type : Poster Presentation

Keywords :

Giant perivascular spaces: a difficult diagnosis?

Annapaola Bocchio ${ }^{1}$, Susanna Cordera ${ }^{2}$, Massimo Rosset ${ }^{1}$, Anna Rosano $^{1}$, Dany Furfaro ${ }^{1}$, Teodoro Meloni ${ }^{1}$

Regional hospital valle d Radiology aosta-Italy ${ }^{1}$ Regional hospital valle d Neurology aosta-Italy ${ }^{2}$ 
Perivascular spaces, also named Virchow-Robin (VR) spaces, are small pial structures, filled with interstitial fluid, surrounding the small vessels as they enter into the brain from the subarachnoid spaces. Occasionally, they may massively enlarge and mimic a cystic mass lesion. Their pathognomonic imaging appearance helps in the diagnosis.

We present the case of a 56 years-old woman, moved from rehabilitation to emergency for sudden worsening of general conditions. At emergency: comatous (GCS 7) Past history: 3 months before, in other hospital, surgery for non-specified cystic brain mass (frontal trans-ventricular craniotomy and partial removal of the lesion). Hystology : normal white matter; few epithelial cells, no mithosis or cellular atypias. CT at emergency: right frontal hypodensity consistent with post-surgery changes and clusters of variably sized cystic cavities in the mesencephalothalamic region, compressing the third ventricle and aqueduct; hydrocephalus. MRI: severe motion artifacts: multiple CSF-like signal mesenchefalic and diencephalic cavities, no changes in the adjacent white matter. Post contrastCT: no enhancement of the mass. Treated for urinary sepsis, the patient recovered and was moved to a rehab-center with residual left hemiplegia. Conclusions: Giant VR spaces may cause mass effect and assume configurations that may be isinterpreted as a cystic brain tumor. When the lesions occur in a characteristic location (along the path of a penetrating vessel), follow CSF signal intensity with all sequences, do not enhance with contrast material, and have normal adjacent brain parenchyma, their appearance is virtually pathognomonic. Still, differentiation between giant VR spaces and cystic brain tumors is sometimes difficult.

\section{1-P.34}

Category: Diagnostic Brain

Presentation Type : Poster Presentation

\section{Keywords :}

\section{Vertebrobasilar ischemia with partial MCA territory infarction in case of a complete ICA agenesis}

Dominik Grieb ${ }^{1}$, Jessica Katharina Baltes ${ }^{1}$, Katharina Melber ${ }^{1}$, Björn Greling ${ }^{1}$, Martin Schlunz-Hendann ${ }^{1}$, Friedhelm Brassel ${ }^{1}$, Dan Meila ${ }^{1}$

Sana Kliniken Duisburg Department of Radiology and Neuroradiology Duisburg-Germany ${ }^{1}$

A 82-year-old man presented to our hospital with recurrent visual disorders of the right eye.

Cranial CT showed infarct demarcation in the posterior third of the left middle cerebral artery (MCA) territory. CCT of the skull base and CTangiography revealed complete absence of the left internal carotid artery (ICA) including the carotid canal. The left anterior cerebral artery (ACA) receives supply from the right hemisphere through the anterior communicating artery (AcomA). The left MCA arises from the vertebrobasilar system through an enlarged posterior communicating artery. There was no vessel occlusion. Additionally, the patient had a saccular AcomAaneurysm.

No hemodynamic relevant stenosis was identifiable, though arterial embolism of the vertebrobasilar system was thought to be the most probable cause of the here underlying left partial MCA territory infarction.

We present the rare case of a complete ICA agenesis with supply of the left MCA through the basilar artery and with supply of the left ACA through the contralateral ICA, respectively (Type A of ICA agenesis according to Lie et al.). Coincidence with intracranial aneurysms is reported.

Normally, patients with these uncommon developmental anomaly of the ICA are asymptomatic. In case of a complete absence of the ICA, vessel occlusion due to atherosclerosis or carotid dissection must be distinguished from a developmental ICA agenesis.

In conclusion, it is crucial to recognize this anomaly, in particular during neurosurgical or neuroendovascular planning and treatment.
1-P.35

Category: Diagnostic Brain

Presentation Type : Poster Presentation

Keywords : Penumbra, contrast, brain perfusion

\section{Importance of MDCT brain perfusion in acute ischemic stroke}

Kristina Davi ${ }^{1}$, Ana Stankovic ${ }^{1}$, Jelena Kostic ${ }^{1}$, Ivan Nikolic ${ }^{2}$, Faris Crnovrsanin $^{1}$, Zivorad Savic ${ }^{1}$, Danilo Stankovic ${ }^{1}$, Dragam Masulovic ${ }^{2}$

Clinical center of Serbia Emergency room neuroradiology BelgradeSerbia ${ }^{1}$ Clinical center of Serbia Radiology and mri Belgrade-Serbia ${ }^{2}$

Introduction: Perfusion $\mathrm{CT}$ is being increasingly used as a diagnostic tool for the evaluation of acute ischemic stroke. It can be performed rapidly and aids in the detection of salvageable tissue (penumbra) from the unsalvageable core infarct.

Methods: CT brain perfusion 4D aids with assessing the a certain part of the brain and evaluating perfusion deficits by displaying 4D-DSA views od blood flow in vessels and 3D perfusion maps. CT perfusion was performed on 160-slice scanner (Toshiba Aquilion Prime) using $50 \mathrm{~mL}$ of nonionic iodinated contrast media (Omnipaque, $370 \mathrm{mg} / \mathrm{mL}$ of iodine; Amersham Heath, Princeton, NJ, USA), injected intravenously at the rate of $5.5 \mathrm{~mL} / \mathrm{s}$ via right antecubital vein followed by $20 \mathrm{~mL}$ of saline at the same rate. Total coverage was $80 \mathrm{~mm}$. The total scan time was approximately $57.7 \mathrm{~s}$. This acquisition also allows generation of intracranial angiographic data as well as perfusion maps. Whole brain non-contrast CT (NCCT) was performed before CTP. Postprocessing was performed on a VitreaCore workstation (Vital Images Inc., MN, USA).

Results: We included patients with acute ischemic stroke admitted to Emergency Room. Cortical involvement was determined on acute NCCT and CTP (T max, cerebral blood volume [CBV], and cerebral blood flow [CBF]). Conclusion: Perfusion CT has proven to be a valuable tool in the diagnosis of acute ischemic stroke. The use of CTP and the advances of this technology will hopefully lead to an extended time window for the use of drugs like tPA for the revascularization of the stroke-affected tissue.

1-P.36

Category: Diagnostic Brain

Presentation Type : Poster Presentation

Keywords : encephalotrigeminal angiomatosis, vascular malformations, calcifications

Correlation between neuroimaging and clinical presentation - way to proper diagnosis

Gordana Milenkovic $^{1}$, Biljana Georgievski-Brkic ${ }^{2}$, Tatjana Stosic-Opincal ${ }^{1}$

General Hospital EUROMEDIK Radiology Obrenovac-Serbia ${ }^{1}$ Special Hospital for cerebrovascular diseases Sveti Sava Radiology Belgrade-Serbia ${ }^{2}$

Background: Sturge-Weber syndrome (SWS) or encephalotrigeminal angiomatosis is sporadic congenital malformation, characterized by angiomatosis vascular malformations of face, eye and central nervous system. Pathoanatomically supstrate of neurological disorders is abnormal development of fetal cortical veins, progressive venous occlusion and chronic venous ischemia, with consequent hypoperfusion of the brain. Main clinical manifestations are seizures, mental retardation and stroke-like episodes.

The Purpose of Presentation: Is to prove importance of correlation between clinical presentation, neurological examination and neuroimaging. Material and Methods: MR imaging was done on GE maschine, type "SYGNA", 1,5 T, with standard protocol for brain examination with postcontrast study.

A Case Report: A 60-years-old women felt left-side weakness few days before. In past history she has seizures and hypertension. CT from the other 
institution showed calcifications at frontal region and hypodensic lesion temporooccipital on the right side. Baseline vital signs: hypertensia. Clinical and neurological status: "port- wine" stains of face, less developed right arm and leg, demaged power of left arm and leg, mental disorientation. Results: MR examination indicated: subacute cerebral ischemia temporooccipital and thalamic on the right side, hemiatrophy of the right cerebral hemisphere, with pachygiria and polymicrogyria of frontoparietal region, amorphous and multiple cortical calcifications ("tram-track" type) with cystic lesions at both sides. MR angiography showed occlusio of the right A. cerebri posterior.

Conclusion: Correlation of neurological, clinical and radiological findings, we have no doubt that this is typical case of Sturge-Weber syndrome.

1-P.37

Category: Diagnostic Brain

Presentation Type : Poster Presentation

Keywords : Infiltrative edema, FLAIR, Gliomas

Infiltrative Edema on FLAIR Images for Detection of Cerebral Gliomas

Slavica Kovacic ${ }_{-}$, Antonija Ruzic Barsic ${ }^{1}$, Damir Miletic ${ }^{1}$, Zoran Rumboldt ${ }^{2}$

University Hospital Rijeka Radiology Viskovo-Croatia ${ }^{1}$ University of Charleston Radiology Charleston -United States ${ }^{2}$

Objectives: To asses the accuracy of fluid-attenuated inversion recovery (FLAIR) images in differentiation of cerebral mass lesions based on the extension of signal abnormality.

Background: Two main types of cerebral edema are vasogenic and cytotoxic. There is another type that resembles vasogenic edema but extends into the gray matter (cortical or deep), which has been called infiltrative edema". It has been proposed that this infiltrative edema may be specific for gliomas.

Methods: FLAIR images from the initial MRI (1.5 T scanner) of 81 patients with definite diagnosis of intra-axial mass lesions (41 gliomas, 31 metastatic neoplasms, 4 abscesses, 3 tumefactive demyelinations, 2 lymphomas) were retrospectively and independently evaluated by 2 readers (neuroradiologists with 2 and 16 years of experience) for the presence of infiltrative edema. The readers were blinded to all clinical information and other MR sequences. Presence of infiltrative edema was considered to be diagnostic for gliomas. Sensitivity and specificity were determined for each reader, and interrater agreement was estimated by kappa coefficient.

Results: Reader A had sensitivity for gliomas of 53.7\% (22/41), specificity was $72.5 \%$. Reader B had sensitivity of $82.9 \%$ (34/41) and specificity of $75.0 \%$. There was a moderate agreement between the readers: Kappa 0.44 (95\% CI 0.25-0.63).

Conclusion: Our results suggest that differentiation of gliomas from other cerebral mass lesions based exclusively on distribution of perilesional signal abnormality on FLAIR images is not very reliable (sensitivity of $83 \%$ or less) and only moderately reproducible

\section{1-P.38}

Category: Diagnostic Brain

Presentation Type : Poster Presentation

\section{Keywords :.}

\section{CT-perfusion of recurrent glial tumors.}

Mikhail Amelin $^{1}{ }^{\text {, Jamil Rzaev }}{ }^{2}$, Sergey Chernov ${ }^{2}$, Andrey Tulupov ${ }^{3}$
Federal Center for Neurosurgery X-ray departament Novosibirsk-Russia ${ }^{1}$ Federal Center for Neurosurgery Surgery Novosibirsk-Russia ${ }^{2}$ Novosibirsk State research University X-ray departament NovosibirskRussia $^{3}$

Purpose: To evaluate changes of perfusion parameters in recurrent glioblastomas and surrounding tissue.

Materials and Methods: In 39 patients (25 male, 14 female, age range 2867 years, follow-up after surgical treatment was 4.5-11 months) with recurrent high-grade glioblastoma (grade III and IV) underwent cerebral perfusion CT on CT unit Siemens Somatom Definintion AS, $50 \mathrm{ml}$ of 350-400 mg I/ml contrast were injected i.v. $5 \mathrm{ml} / \mathrm{s}$, duration of scan was $50 \mathrm{~s}$, using jog-scan, Z-range was $80 \mathrm{~mm}$, tube voltage $80 \mathrm{kV}, 140 \mathrm{mAs}$. Postprocessing was performed with Siemens Volume perfusion CT software. Cerebral blood flow (CBF), cerebral blood volume (CBV), time to peak (TTP), mean transit time (MTT), and permeability (PMB) were measured in the tumor and peritumoral tissue.

Results: In all patients tumor tissue has highly elevated CBV (up to 6.50 $\pm 4.56 \mathrm{ml} / 100 \mathrm{~g}$ ) and $\mathrm{CBF}$ (up to $75.32 \pm 3.76 \mathrm{ml} / 100 \mathrm{~g} / \mathrm{min}$ ), these parameters were the higher, the higher was tumor grade comparing to normal brain tissue and peritumoral tissue. TTD, MTT and TTP were slightly elevated in tumor tissue and peritumoral tissue comparing to normal brain tissue $(8.18$ $\pm 1.27,7.44 \pm 1.22,11 \pm 1.61 \mathrm{~s})$. Permeability was also highly elevated in tumor tissue (up to $23.66 \pm 6.41 \mathrm{ml} / 100 \mathrm{~g} / \mathrm{min}$ ), the higher was the grade the more elevated was permeability, in surrounding peritumoral tissue it was slightly elevated $(1.18 \pm 0.19 \mathrm{ml} / 100 \mathrm{~g} / \mathrm{min})$ comparing to normal tissue $(0.84 \pm 0.09 \mathrm{ml} / 100 \mathrm{~g} / \mathrm{min})$.

Conclusion: In patients with recurrent glioblastomas perfusion parameters in tumor and peritumoral tissue have correlation with tumor grade.

1-P.39

Category: Diagnostic Brain

Presentation Type : Poster Presentation

Keywords : central blockades. spine

Looking at the spine in a different way: radiological anatomy and its importance in central blockades.

Patricia Martin ${ }^{1}$, Elena Salvador ${ }^{1}$, Amaya Hilario ${ }^{1}$, Laura Koren ${ }^{1}$, Federico Ballenilla ${ }^{1}$, Jorge Hernando ${ }^{2}$, Ana Ramos ${ }^{1}$

Hospital 12 De Octubre Radiology Madrid-Spain ${ }^{1}$ Hospital De San Juan Anesthesiology Alicante-Spain ${ }^{2}$

Teaching Points:

- To review the radiological anatomy of the spinal region.

- To explore the notions of lumbar puncture and central blockade techniques.

- To describe the possible variants and pathologies those have to be considered when performing these techniques.

Outline: Currently regional anesthesia is becoming the technique of choice, especially in abdominal and inferior extremities surgery.

In order to achieve a secure and successful central blockade is mandatory a deep knowledge of the spinal anatomy:

- To localize the optimum site for the puncture procedure.

- To recognize the features of the structures that the needle have to go through for guiding it correctly and to predict the difficulties that this structures could show.

- To know the space in which the anesthetic solution will spread and possible barriers that could exist (for example, to determinate the optimum dose). 
Radiological techniques provide an excellent anatomical representation of the spinal region. They allow us to evaluate bone structures, vertebral canal and its components, and also perform myelography studies.

Conclusions: The knowledge about radiological spinal anatomy and its applications in regional anesthesia will allow us to recognize variants and pathologies that may have implications in the puncture procedure as well as in the dissemination of the anesthetic solution.

\section{1-P.40}

Category: Diagnostic Brain

Presentation Type : Poster Presentation

\section{Keywords :}

\section{MR Characteristics of Epidermoid Cysts}

Gordana Tošović ${ }^{1}$, Dragan Stojanov ${ }^{1}$, Slađana Petrović ${ }^{1}$, Ivana Marković $^{1}$, Nikola Stojković ${ }^{1}$

\section{Clinical center Niš Radiology Niš-Serbia ${ }^{1}$}

Introduction: Epidermoid cysts are benign slow-growing, mostly congenital lesions. The most frequent localizations are cerebellopontine angle, parasellar cistern and the fourth ventricle. In determining boundaries of the tumor and relationship with the surrounding structures an MRI examination is exceptionally useful.

Aim: Presenting characteristic MRI findings by examining three patients with intracranial epidermoid cysts.

Methods: Two male patients and one female patient were examined with the Siemens Avanto unit of the strength of 1.5 T. Spin echo sequences were used (T1W, T2W, T1W+C), FLAIR, DWI and ADC. A gadoliniumbased contrast medium was applied intravenously in the dosage of $0.2 \mathrm{ml} /$ $\mathrm{kg}$ of the bodily weight.

Results: All three lesions showed low signal intensity on T1W and high signal intensity on T2W. In the FLAIR sequence, the lesions were heterogeneous, two of which were hypointense relative to gray matter, and hyperintense relative to the CSF, while the third cyst was hyperintense. After administration of the contrast material, there were no signals alteration in the first two described lesions, while the third one displayed a minimum enhancement of the septa. All three lesions demonstrated significant restricted diffusion.

Conclusions: The lack of contrast enhancement is a significant radiological characteristic of epidermoids. The FLAIR sequence is more reliable than the SE sequences in detecting intracranial epidermoids, showing a variable, but satisfactory, contrast in relation to the liquor and the cerebral parenchyma. The hyperintensity on the DWI and the hypointensity on the ADC map enable a more precise diagnosis.

\section{1-P.41}

Category: Diagnostic Brain

Presentation Type : Poster Presentation

Keywords : Neurofibromatosis type 1, Moya Moya syndrome

Laughter induced hemiparesis in a patient with Neurofibromatosis type 1 .

Anna Drazyk ${ }^{1}$, Alan O'Hare ${ }^{2}$, Ronan Killeen ${ }^{3}$, M Javadpour ${ }^{4}$, Chris McGuigan ${ }^{1}$

St Vincent's University Hospital Neurology Dublin-Ireland ${ }^{1}$ Beaumont Hospital Neuroradiology Dublin-Ireland ${ }^{2}$ St Vincent's University Hospital Neuroradiology Dublin-Ireland ${ }^{3}$ Beaumont Hospital Neurosurgery DublinIreland $^{4}$
Neurofibromatosis type 1 is a genetic multisystem disease. It has a wide range of manifestations with cutaneous, neurological and musculoskeletal being the most common.

Vascular complications are less prevalent but increasingly recognized. Progressive internal carotid artery stenosis resulting in Moya Moya syndrome is the most common cerebrovascular abnormality in NF 1 .

An 18-year-old woman with background history of Neurofibromatosis type 1 presented to Emergency Department with a six-month history of episodes of transient right sided weakness, increasing in frequency. Intensive laughter was the only trigger for each event. An initial CT scan of brain was normal. MR Angiogram (MRA) revealed stenosis of the left ICA with formation of extensive collaterals vessels around the circle of Willis.

An MRI Perfusion scan revealed significant hypoperfusion of the territory of the left ICA.

A cerebral angiogram confirmed extreme stenosis of left internal carotid artery with occlusion of ipsilateral middle and anterior cerebral arteries.Posterior circulation was also affected with severely stenosed distal portion of basiliar artery.

Perfusion of the left hemisphere was fully dependent on collaterals from external carotid artery and contralateral MCA. Patient underwent successful surgical revascularization.

Vascular imaging should be an important consideration in approaching patients with Neurofibromatosis type 1. In this case unusual presentation lead to a diagnosis and successful treatment of this, otherwise potentially devastating, condition.

\section{1-P.42}

Category: Diagnostic Brain

Presentation Type : Poster Presentation

Keywords : MR Spectoscopy, glial tumors, creatin

Investigation of Creatin Levels in Low and High Grade Glial Tumors Using mr Spectroscopy

Mustafa Nadir ${ }^{1}, \underline{\text { Emel Ada }}{ }^{1}$, Agah Baran ${ }^{1}$, Ayca Ersen Danyeli ${ }^{2}$, Nuri Karabay

Dokuz Eylul University Radiology Izmir-Turkey ${ }^{1}$ Dokuz Eylul University Medical Pathology Izmir-Turkey ${ }^{2}$

Objective: To describe the creatine changes in single and multivoxel MRSpectroscopy (MRS) in patients with glial tumor.

Materials and Methods: The patients with a glial tumor diagnosed between 07-2010 and 12-2015 were evaluated retrospectively. Among 32 patients, 20 patients had single voxel MRS from tumoral and contralateral sites, 20 patients had multivoxel. In single voxel study, $\mathrm{Cr}$ (area) and $\mathrm{Cr}$ (height), $(\mathrm{Cho}+\mathrm{Cr}) / \mathrm{NAA}$ and Cho/NAA from tumoral site were compared to contralateral normal site. In multivoxel study, the highest and lowest $\mathrm{Cr}$ (height) containing voxels in tumoral area were noted, $\mathrm{Cr}(\mathrm{h})$ and $\mathrm{Cho} / \mathrm{NAA}$ values were noted and compared with symetric contralateral side. Dataset were evaluated using Kolmogorov-Smirnov test and paired-samples t-test. Pearson correlation test were used to asses the correlation between values. Results: No correlation was found between $\mathrm{Cr}(\mathrm{a})$ and $\mathrm{Cr}(\mathrm{h})$ measurements in single voxel MRS (p:0,104 and 0,553 respectively). The $(\mathrm{Cho}+\mathrm{Cr}) / \mathrm{NAA}$ and Cho/NAA rates in the SV study showed statistically significant difference (p:0 and 0 respectively). In multivoxel study the comparison of lowest $\mathrm{Cr}(\mathrm{h})$ values showed no significant difference $(\mathrm{P}=0,131)$. But significant difference was noted in the highest $\mathrm{Cr}(\mathrm{h})$ $(\mathrm{P}=0,003)$. Cho/NAA ratios were significantly different between low $\mathrm{Cr}(\mathrm{h})$ containing and high $\mathrm{Cr}(\mathrm{h})$ containing groups $(\mathrm{p}=0)$. The $\mathrm{Cho} /$ NAA ratios were significantly higher in low $\operatorname{Cr}(\mathrm{h})$ areas $(\mathrm{p}=0)$.

Conclusion: The creatin levels varied inside tumor tissue. In the areas with low $\mathrm{Cr}(\mathrm{h})$ values, the Cho/NAA ratios were significantly higher, indicating a higher tumoral grade area. 
1-P.43

Category: Diagnostic Brain

Presentation Type : Poster Presentation

Keywords : fractional anisotropy, mean diffusivity, diffusion tensor imaging, resting state functional MRI

The Neuroimaging Investigation in Brain of Alzheimer's Disease Patients Without and With Depression

Clayton Chi-Chang Chen ${ }^{1}$, Jyh-Wen Chai ${ }^{1}$, Ni-Jung Chang ${ }^{1}$, Ching-Po Lin ${ }^{2}$, Hsian-Min Chen

Taichung Veterans General Hospital Department of Radiology TaichungTaiwan ${ }^{1}$ National Yang Ming University Institute of Neuroscience Taipei-Taiwan ${ }^{2}$ Taichung Veterans General Hospital Department of Medical Research Taichung-Taiwan ${ }^{3}$

Purpose: This study elucidate the brain tissue difference between Alzheimer's disease (AD) patients without (ADND) and with (ADD) depressive symptoms in the brain volume analysis, fractional anisotropy (FA) with mean diffusivity (MD) indices of white matter (WM) diffusion tensor imaging (DTI) alternations and changes of functional connectivity (FC) in resting state functional MRI (rs-fMRI).

Materials and Methods: In this study, twenty ADND and twenty-six ADD patients were enrolled. The routine MRI and the DTI images with FA and MD were obtained. The rs-fMRI data were acquired with voxel-base measurement of $\mathrm{FC}$ of resting fMRI signal.

Results: Concerning regional brain volume analysis, WM volume fraction in the sublobar region of the ADND patients was significantly lower than that of the ADD patients. The DTI indices revealed higher global FA and lower global MD in ADD than ADND patients. Analysis of regional WM integrities, ADD patients appeared higher FA in frontal and sublobar region, and lower MD indices over frontal, limbic, midbrain and sublobar region than ADND patients. In the rs-fMRI study, the ADND vs ADD patients show increased default-mode network activity in anterior prefrontal cortex, middle frontal cortex and subgyral area.

Conclusions: The structural connections found from DTI and the functional correlations from rs-fMRI show the overlap within the DMN areas. The investigation of morphometric analysis, DTI alternation and rs-fMRI functional connectivity is helpful in revealing the difference of brain abnormalities in $\mathrm{AD}$ without and with depression.

\section{1-P.44}

Category: Diagnostic Brain

Presentation Type : Poster Presentation

Long-term biodistribution and excretion of gadolinium-based contrast agents (GBCAs)

Eric Lancelot $^{1}$

Guerbet Clinical Development Roissy CDG Cedex-France ${ }^{1}$

Purpose: To ascertain the existence of a deep compartment for gadolinium storage in the body and assess whether the GBCAs used in Neuroradiology present the same pharmacokinetic profile.

Material and Methods: A meta-analysis of the available clinical and preclinical pharmacokinetic data about gadolinium in plasma, urine and bone was performed. The rate constants of the distribution (alpha), elimination (beta), and residual excretion (gamma) phases were determined from the plasma concentration and the renal excretion rate time curves. Gadolinium concentrations in murine bone and bone marrow were also studied.

Results: All GBCAs displayed a residual excretion phase of gadolinium in healthy volunteers. Gadoteric acid was cleared five times faster than linear
GBCAs (gamma $=0.107$ h- 1 vs. $0.020 \pm 0.008$ h-1, respectively). Similar results were obtained from animal studies. A strong correlation was shown between the gamma values of the GBCAs and their thermodynamic stability constants $\left(\mathrm{R}^{2}=0.9764\right)$. Faster gadolinium clearance from murine bone was also observed with gadoteric acid and gadoteridol (0.131-0.184 day-1) than with linear GBCAs (0.004-0.067 day-1). The concentration ratio between bone marrow and bone matrix increased less with gadoteric acid than with gadodiamide (1.9 vs. 6.5 at 4 hours, respectively).

Conclusion: This meta-analysis revealed that all GBCAs present a residual excretion, reflecting the existence of a deep compartment of distribution. Gadoteric acid undergoes a much faster residual excretion than linear GBCAs. The bone may represent a reservoir for gadolinium dissociation, long-term accumulation, and slow release into the blood stream, especially when using linear GBCAs.

\section{1-P.45 \\ Category: Diagnostic Brain \\ Presentation Type : Poster Presentation}

Leptomeningeal score (LMs) on Computed Tomography Angiography and outcome predictors in Acute Ischemic Stroke (AIS).

$\underline{\text { Edoardo Puglielli }}{ }^{1}$, Ruggero Lattanzi ${ }^{1}$, Gianluigi Esposito ${ }^{1}$, Vincenzo di Egidio

Asl Teramo Interventional Radiology Teramo-Italy ${ }^{1}$

Purpose: Aim of this study was to systematically review the importance of LMs in the outcome after AIS and identify a practical and reliable score system for grading collateral flow.

Methods: 195 consecutive patients with AIS (mean age 55,7y, April 2009-May 2016), classic on-set, undergoing to endovascular multimodal treatment after basal CT and Computed Tomography Angiography. The LMs is based on double blinded scoring pial and lenticulostriate collaterals arteries ( 0 , no; 1 , less; 2 , equal or more prominent compared with matching region in opposite hemisphere) in 6 ASPECTS regions (M1-6) plus anterior cerebral artery region and basal ganglia. Good clinical outcome was defined as $\mathrm{mRS} \leq 2$ at 90 days.

Results: CTA can well define the occlusion site, depict arterial state, grade collateral blood flow, and characterize atherosclerotic status. In our series 128/195 (65.64\%) had a good (17-20), 45/195 (23.07\%) a medium (11-16), and 22/195 $(11,28 \%)$ a poor $(0-10)$ LMs. Interclass correlation coefficient of $0.87(95 \% \mathrm{CI}, 0.77 \%-0.95 \%)$ in the two readers. In multivariable analysis, the LMs (good versus poor: OR, 15.8 ; $95 \%$ CI, 3.3\%-97.4\%; medium versus poor: OR, $9.5,95 \% \mathrm{CI}$, $1.7 \%-50.6 \%)$, age $(<80$ years), baseline ASPECTS $(\geq 8)$, and CTP were independent predictors of good clinical outcome.

Conclusion: LMs appear to be as a strong imaging parameter for predicting clinical outcomes in patients with AIS and can therefore be used for imaging based patient selection.

\section{1-P.46}

Category: Diagnostic Brain

Presentation Type : Poster Presentation

Posterior circulation and Computed Tomography Perfusion (CTP): is it a valid tool for Acute Ischemic Stroke (AIS) patients selection?

Edoardo Puglielli ${ }^{1}$, Ruggero Lattanzi ${ }^{1}$, Gianluigi Esposito ${ }^{1}$, Federica de Filippis ${ }^{1}$, Vincenzo di Egidio ${ }^{1}$

Asl Teramo Interventional Radiology Teramo-Italy ${ }^{1}$ 
Purpose: Embolectomy in AIS has changed the goals of neuro-imaging and CTP already plays a pivotal role in the diagnosis and selection of patients eligible for treatment.

Methods: 75 consecutive patients with posterior AIS (mean age 53,7y, April 2009-May 2016), with classic onset, undergoing to endovascular treatment after CTP evaluation. A false delayed peak enhancement or increased mean transit time in a region with normal or only slightly abnormal cerebral blood volume was suggestive for an ischemic penumbra that leading to a false appearance of penumbra. Clinical history and findings at basal and CT Angio (CTA) and/or DSA was correlated with PCT data with a regression analysis $(p<0,005)$. Good clinical outcome was defined as $\mathrm{mRS} \leq 2$ at 90 days.

Results: CTA can well define the occlusion site, depict arterial state, grade collateral blood flow, and characterize atherosclerotic status (73.33\% [55/ 75]), whereas CTP accurately delineates the infarct core and the ischemic penumbra $(64.04 \%$ [48/75]). False penumbra, each of which produces a different pattern at imaging were: vascular dysregulation $(2,66 \%[2 / 75])$, upstream flow restriction (17.33\% [13/75]), evolution of ischemic change (5.33\% [4/75]), positioning of the patient's head at an angle during image acquisition $(5.33 \%$ [4/75]), variant anatomy in the circle of Willis $(9,33 \%$ [7/75] and bone artefact 8/75 [10.66]).

Conclusion: Familiarity with the imaging patterns and causes of posterior circulation false penumbras may increase the radiologist's confidence in the diagnosis of AIS.

\section{1-P.47}

Category: Diagnostic Brain

Presentation Type : Poster Presentation

Keywords : MRI, Spectroscopy, brain tumor, misinterpretation

\section{Misinterpretation of MR Spectroscopy in the Preoperative Diagnosis of Brain tumors}

Eftychia Kapsalaki ${ }^{1}$, Ioannis Tsougos ${ }^{2}$, George Kyrgias ${ }^{3}$, Kostas Fountas ${ }^{4}$, Ioannis Fezoulidis ${ }^{1}$

Univ of Thessaly Radiology Larissa-Greece ${ }^{1}$ Univ of Thessaly Medical Physics Larissa-Greece ${ }^{2}$ Univ of Thessaly Radiation Oncology LarissaGreece ${ }^{3}$ Univ of Thessaly Neurosurgery Larissa-Greece ${ }^{4}$

Introduction: MR-spectroscopy (MRS) is an advanced diagnostic MR technique evaluating the metabolic profile of a brain lesion. The purpose of our study is presenting brain tumors that have been misdiagnosed with MRS.

Material and Methods: In our retrospective clinical study we included 212 consecutive patients (112 male, 100 female) with a brain tumor. All patients underwent an MRS within 2 weeks of their initial imaging diagnostic study. Surgical biopsy or excision of the brain tumor was performed with 10 days of the performed MRS. MR Spectroscopy was performed at a 3T MR scanner (GE Medical Systems) using sinlge voxel and CSI methodology.

Results: The diagnostic accuracy of MRS was $91 \%$. In 19 patients, MRS misinterpreted the diagnosis even under taking into consideration other advanced imaging studies (perfusion, diffusion). Misinterpretation included lymphoma in 2 patients, gliosarcoma in 1 patient, tuberculoma in 1 case, meningioma in one patient, metastatic tumor in 1 patient and in 13 patients with gliomas, imaging findings of MRS misinterpreted the grade of the glioma. The factors that on a retrospective basis were responsible for the misdiagnosis included contamination from the surrounding tissue, the evaluation of only long or only short TE values and the misinterpretation of the Myoinositol peak.

Conclusions: MR-S ia a valuable tool in the diagnosis of brain tumors. However it needs to be performed lege artis and should be used complementarily with other advanced imaging MR techniques before contributing to the final diagnosis.
1-P.48

Category: Diagnostic Brain

Presentation Type : Poster Presentation

Keywords : fMRI, BOLD, language, gliomas, cavernous angioma, epilepsy

Cortical Reorganization of Language Function Induced by Brain Lesions: an Fmri Study

Loris Di Clemente ${ }^{1}$, Valentina Panara ${ }^{1}$, Ioan Paul Voicu ${ }^{1}$, Riccardo Navarra ${ }^{1}$, Roberto Guidotti ${ }^{1}$, Peter A. Mattei ${ }^{1}$, Massimo Caulo ${ }^{1}$

Università degli studi “G. D Department of neuroscience and imaging Chieti-Italy ${ }^{1}$

Introduction: The language network is distributed in two principal brain regions, the frontal and temporo-parietal lobes classically defined as Broca's and Wernicke's areas. The distribution of these regions varies spatially between subjects. The presence of a brain lesion may increase this variability. This study aims to investigate the effects of different types of brain lesions on the cortical reorganization of the language function.

Materials and Methods: We retrospectively evaluated 17 healthy subjects (controls) and patients with a left hemisphere cavernous angioma (n.13), small glioma (n. 21) and temporal lobe epilepsy (n. 9) who underwent a verb generation fMRI study for pre-surgical mapping. The activation coordinates in the left inferior frontal gyrus (Broca's region), temporo-parietal junction and superior temporal sulcus (Wernickes region) were determined for each subject. Cluster dispersion and distances of the activations from those of the control group were calculated for the three cortical regions for each group of patients. The statistical significance of the cluster dispersion was assessed using permutation tests $(n=2000)$.

Results: The dispersion index was significantly lower in the control group in Broca's and Wernicke's regions and in patients with cavernous angioma in Broca's region $(p<0,05)$. Patients with gliomas presented the higher dispersion of activation areas.

Conclusions: The presence of a glioma in the left hemisphere determined a cortical reorganization of language functional area.

\section{1-P.49}

Category: Diagnostic Brain

Presentation Type : Poster Presentation

Keywords : SVS, stroke

SVS : inter MRI manufacters agreement, inter and intra observers agreement, histo-MR correlation

Bourcier Romain ${ }^{1}$, Détraz Lili ${ }^{1}$, Guyomarch Béatrice ${ }^{2}$, Toquet Claire ${ }^{3}$, Serfaty Jean Michel ${ }^{4}$, Desal Hübert ${ }^{1}$

Centre Hospitalier Universitaire, Hôpital R et G Laënnec Diagnostic and Interventional Neuroradiology Nantes-France ${ }^{1}$ Centre Hospitalier Universitaire, Hôpital R et G Laënnec Centre d'Investigation clinique Thorax Nantes- ${ }^{2}$ Centre Hospitalier Universitaire, Hôtel Dieu Pathological Anatomy and Cytologie Department Nantes- ${ }^{3}$ Centre Hospitalier Universitaire, Hôpital R et G Laënnec Diagnostic Cardiac and Vascular Imaging Nantes- ${ }^{4}$

Background and Purpose: The susceptibility vessel sign (SVS) on gradient echo T2 weighted is known to indicate an erythrocyte-rich thrombus. The purpose of this study was to evaluate inter MR imaging agreement, inter and intra observer agreements and histo- MRimaging correlation of this sign. 
Materials and Methods: 35 clots with different red blood cell (RBC)/fibrin ratios were made from fresh ovine blood and underwent a gradient echo $\mathrm{T} 2$ weighted based on the constructor parameters on machines of four different MRI manufacturers (1, 5 T GE, Siemens, Philips, 3T Philips). 10 radiologists have classified twice, the SVS of each clot's image in three groups: absent, doubtful, present. A three levels classification was used by the histologist to quantify the RBC proportion in each clot after MRI. Results: The inter MRI manufacturers agreement was moderate $\mathrm{k}=0,44$ (IC $[0,373-0,422])$ to good $\mathrm{k}=0,671$ (IC $[0,606-0,740])$. The inter observers agreement was good $\mathrm{k}=0,541$ (IC $[0,446-0,636]$ ) to excellent $\mathrm{k}=0,883$ (IC $[0,827-0,940])$. The intra observers agreement was also good to excellent $\mathrm{k}=0,852$ (IC [0,787 - 0,917]). The histo-MR imaging correlation was excellent: $\mathrm{k}=0,00$ (IC $[0,00-0,00]$ ).

Conclusions: Our study suggests that the SVS is a reproducible sign between observers and highly correlated to the histology composition despite a moderate to good inter MRI manufacturers agreement. Therefore, interpretation of this sign in large studies using different machines of MRI manufacturers and parameters has to be done with care.

\author{
1-P.50 \\ Category: Diagnostic Brain \\ Presentation Type : Poster Presentation
}

Keywords : magnetic resonance spectroscopy, $\mathrm{N}$-acetylaspartate, gender differences, educational differences

Correlation between $\mathrm{N}$-acetylaspartate as Neuronal Biomarker with Education Level in Healthy Adults

Alma Brakus ${ }^{1}$,- Sanja Stojanovic ${ }^{1}$, Dusko Kozic ${ }^{2}$, Vedran Žigić ${ }^{1}$, Biljana Georgievski-Brkic ${ }^{3}$, Jelena Ostojic ${ }^{1}$

Clinical Centre of Vojvodina Centre for Radiology, Novi Sad-Serbia ${ }^{1}$ Oncology Institute of Vojvodina, Sremska Kamenica Center for Imaging Diagnostics Novi Sad-Serbia ${ }^{2}$ Special Hospital for Cerebrovascular Disease "Sveti Sava" Belgrade-Serbia ${ }^{3}$

Background: MRS allows for interrogation of biochemical substrates of brain structure and examination of changes that are not visible on routine neuroimaging. $\mathrm{N}$-acetylaspartate (NAA) is metabolite found primarily within neurons and therefore considered as a marker of neuronal integrity. We hypothesized that in healthy subjects its whole brain concentration may be related to formal educational attainment.

Objective: To determine whether $\mathrm{N}$-acetylaspartate (NAA), a metabolite found primarily within neurons, is related to gender and educational in healthy men and women by multivoxel magnetic resonance spectroscopy (MRS).

Materials and Methods: We performed multivoxel magnetic resonance imaging and spectroscopy in 50 healthy volunteers ( 27 women and 23 men) using 1.5T scanner. Spectra from 12 different voxels were obtained, covering frontal, paracentral, and parietal white and gray matter. Three dominant signals were analyzed: NAA, $\mathrm{tCr}$ and Cho, and expressed as ratios of Cho/tCr, NAA/tCr, NAA/Cho. Six voxels were in bilateral anterior, middle and posterior regions containing predominantly white matter (voxel numbers $1,2,5,6,9,10$ ) and six voxels were in bilateral mesial cortex with mostly gray matter of the anterior, middle and posterior regions.

Results: We found statistically significant difference betweengroups in $\mathrm{NAA} / \mathrm{Cr}$ metabolites ratio in threee location showed greater NAA/Cr in sections of the superior longitudinal fasciculus, compared to adults with lower education. There was no statistically significant difference in NAA/Cho ratio between men and women.
1-P.51

Category: Diagnostic Brain

Presentation Type : Poster Presentation

Keywords : CT, DECT, brain, contrast

Single Brain Post-Contrast Dual-Energy Computer Tomography Acquisition Replacing Pre- and Post-Contrast Acquisitions

Nadya Pyatigorskaya ${ }^{1}$, Bert Geerts ${ }^{2}$, Bruno Law Ye ${ }^{1}$, Damien Galanaud ${ }^{1}$, Didier Dormont ${ }^{1}$

Pitié Salpêtrière Neuroradiology Paris-France ${ }^{1}$ Ghent University Hospital Radiology Radiology Ghent-Belgium ${ }^{2}$

Introduction: In a routine brain CT investigation, when contrast injection is required, the non-contrast acquisition is performed to detect spontaneous high densities. However, this double acquisition is a source of increased irradiation. Our study was aimed at assessing the image quality and diagnosis performances of the virtual non-contrast images generated after post-contrast dualenergy CT (DECT) as compared to the standard non-contrast acquisition.

Methods: 105 subjects with normal CT and 81 subjects with brain lesions were retrospectively included. For all the patients, single-energy noncontrast CT and contrast-enhanced DECT (Discovery CT 750HD; GE Healthcare, Milwaukee, Wis) images had been obtained. Virtual noncontrast images were secondarily obtained. Two neuroradiologists, blinded to the patients' data, analyzed the images on the basis of visual scale for image quality, subtraction quality, and confidence. The density (HU) was measured in the normal parenchyma and in a lesion, if present. Results: The image quality, subtraction quality, and confidence rating were good for virtual non-contrast images. There was no significant deviation of the HU measurements in virtual non-contrast images from those in non-contrast ones. Moreover, in the case of a lesion, significant enhancement was observed in post-contrast images as compared to virtual non-contrast ones $(\mathrm{p}<0.001)$. All the lesions were correctly characterized with both sets of images.

Conclusion: The image quality and diagnosis performance of the virtual non-contrast images generated after contrast injection were found to be satisfactory. Thus it might be suggested that diagnosis can be made on the basis of post-contrast images, using DECT, without non-contrast acquisition.

\section{1-P.52}

Category: Diagnostic Brain

Presentation Type : Poster Presentation

CT Angiography Follow-up in the Endovascular Aneurysm Treatment : Early and Late Results

Ozden Demir ${ }^{1}$, Volkan Cakir ${ }^{2}$, Melda Apaydin ${ }^{2}, \underline{\text { Fazil Gelal }}^{2}$

IKCU IAEAH Radiology IZMIR-Turkey ${ }^{1}$ IKCU IAEAH Radioloy IZMIR-Turkey $^{2}$

We aimed to compare clinical and anatomical results of endovascular treated intracranial aneurysms (IA) in computed tomographic angiography (CTA) with digital subtraction angiography (DSA).

A total of 40 (mean age $53, \mathrm{~F} / \mathrm{M}=25 / 15)$ ) out of 78 patients in the endovascular treatment of intracranial aneurysms were evaluated. 38 patients were excluded for different reasons. The aneuysms were mostly located in ICA [26 (65.0\%)], median size was $6 \mathrm{~mm}$, type was mostly $34(85.0 \%)$ saccular. The patients's DSA and CTA studies were done in 3.,6..,12. month after the endovascular treatment procedure. Early and late angiographic data, recurrence, known changes in the residue, de novo aneurysm were evaluated. CT and DSA were compared each other for the diagnosis of residual or complete occlusion. 
CTA detected no residua in 38(95\%) patients, $2(5.0 \%)$ patients had residua. The DSA results are analyzed $36(90.0 \%)$ patients had no residua, and $4(10.0 \%)$ patients had residual presence. In the analysis results of the two methods it was found to be compatible with each other good $($ Kappa $=0.643, p=0.008)$. Sensitivity to detect the presence of residual CT results of $50 \%$, a specificity of $100 \%$ and an accuracy rate was calculated as $95 \%$.

CTA seems to evaluate clinical and anatomical findings after the endovascular treated anuerysms efficiently and non-invasively.

\author{
1-P.53 \\ Category: Diagnostic Brain \\ Presentation Type : Poster Presentation
}

Keywords : cardioembolic stroke, SWI, Clot, IV rTPA

\section{Importance of Susceptibility-Weighted Imaging for examining the clot and its lytic response in Acute Cardioembolic Stroke, single cen- ter experience}

\section{Ossama Mansour Mansour ${ }_{-}^{1}$, Mohamed Saher ${ }^{2}$}

Alexandria University Neurology and Neuroradiology Alexandria-Egypt ${ }^{1}$ Cairo University Neurology Cairo-Egypt ${ }^{2}$

Introduction: Susceptibility-weighted imaging (SWI) can show an intravascular thrombus as a susceptibility vessel sign (SVS). hereby, we studied the usefulness of SWI in the detection of an intravascular thrombus and its lytic response to IVrTPA in acute ischemic stroke of cardiac source by comparing the SVS in SWI to the vessel status on TOF magnetic resonance angiography (MRA).

Methods: In this study, We enrolled patients with cardioembolic stroke in the anterior circulation within 24 hours from stroke onset. The frequency and location of the SVS on SWI were compared with those of occlusion on MRA.

Results: One hundred patients were enrolled in this study. The SVS was observed in $75 \%(75 / 100)$ of the enrolled patients while not observed in $25 \%(25 / 100)$ SVS -ve group of patients. MRA showed occlusion in $57 \%(57 / 100)$ of the enrolled patients. The SVS was identified in all 75 patients with occlusion on MRA. The SVS was observed in $18(31.6 \%)$ of 57 patients without occlusion on MRA $(\mathrm{P}<0.05)$, which was identified mainly in post-bifurcation segments of the middle cerebral artery. in the SVS+ve group of patient 50 patients $(50 / 75)$ received IVrtpa with good outcome (mRS $0-2$ ) was achieved in $76 \%$ at mean follow up 45 days. While in SVS -ve patient group $20(20 / 25)$ patients received thrombolysis with good outcome in $35 \%$ of them.

Conclusions: SWI is a useful tool to determine the thrombus location and multiplicity, especially in distal intracranial arteries, additionally it predict good outcome to thrombolysis specially in carioembolic stroke.

\section{1-P.54}

Category: Diagnostic Brain

Presentation Type : Poster Presentation

Keywords : Intracranial calcifications,computed tomography

\section{Intracranial calcifications}

Dejan Kostic ${ }^{1}$, Aleksandar Jovanovski ${ }^{1}$, Smiljana Kostic ${ }^{2}$, Predrag Peric $^{3}$, Biljana Georgievski-Brkic ${ }^{4}$, Miroslav Misovic ${ }^{1}$, Tijana Kosanovic $^{1}$

Military Medical Academy Institut of radiology Belgrade-Serbia ${ }^{1}$ Military Medical Academy Clinic for neurology Belgrade-Serbia ${ }^{2}$
Military Medical Academy Clinic for neurosurgery Belgrade-Serbia ${ }^{3}$ Special Hospital for Cerebrovascular Diseases, Sveti Sava radiology, Belgrade-Serbia ${ }^{4}$

The presence of intracranial calcifications is the most common finding in the head computed tomography (CT), which is very sensitive for the detection and localization of the above mentioned. Those findings can be random (casual), but can also be significant for the diagnosis of a pathological disease.

Intracranial calcifications can be grouped into 8 groups according to their etiopathogenesis: physiological, congenital, posttraumatic, metabolic and endocrine, vascular, infectious, inflammatory and tumor.

With the CT images of heads of various patients with intracranial calcifications we want to point out that their distribution as well as appearance compared with clinical picture and biochemical analysis of blood can significantly contribute to the accurate diagnosis.

\section{1-P.55}

Category: Diagnostic Brain

Presentation Type : Poster Presentation

Keywords : Multimodal, MR Imaging, brain lesions, glioma, metastases, bioimaging

Mutlimodality MR imaging for differentiation between brain tumor lesions

Faris Durmo ${ }^{1}$, Anna Rydelius ${ }^{2}$, Silke Engelholm ${ }^{3}$, Sara Kinhult ${ }^{3}$, Krister Askaner ${ }^{4}$, Jimmy Lätt ${ }^{5}$, Pia C Sundgren ${ }^{6}$

Institution of Clinical Sciences Lund University Kristianstad-Sweden ${ }^{1}$ Institution of Clinical Sciences Department of Neurology, Skane University Hospital, Lund University Lund-Sweden ${ }^{2}$ Institution of Clinical Sciences Department of Oncology, Skane University Hospital, Lund University Lund-Sweden ${ }^{3}$ Center for Imaging and Function Department of Medical Imaging and physiology, Skane University Hospital, Lund University Malmö-Sweden ${ }^{4}$ Center for Imaging and Function Department of Medical Radiation Physics, Skane University Hospital, Lund University Lund-Sweden ${ }^{5}$ Institution of Clinical Sciences Department of Radiology, Skane University Hospital, Lund University Lund-Sweden ${ }^{6}$

Purpose: Applying diffusion and perfusion metrics for evaluation of low-(LGG), high grade glioma (HGG) and metastases (MET) for differential diagnosis.

Materials and Method: 43 patients (18 HGG, 10 LGG, and 15 MET) were included. MR data for tumour volume, perilesional edema, rCBF-, rCBV-, FLAIR-, FA-, ADC- maps were quantified by regions of interest (ROI). Measures of different parameters, and ratios, using contralateral white matter as denominator, were performed. A binary logistic regression model was constructed for multi-parametric analysis and ROCanalysis.

Results: Significant difference was found for nADCt, rCBF, rCBV between LGG and HGG, nADCe between HGG and MET, and Ev, Ev-Tv ratio, nADCt, nADCe, rCBF, rCBV between LGG and MET. ROCanalysis for HGG compared to LGG showed $80 \%$ sensitivity and 81.2 $\%$ specificity for nADCt, $100 \%$ sensitivity and $100 \%$ specificity for $\mathrm{rCBF}$ and $80 \%$ sensitivity and $90 \%$ specificity for rCBV. ROC-curves between MET and LGG showed sensitivity and specificity for Ev $73.3 \%$ and $90 \%$, Ev-Tv ratio $80 \%$ and $100 \%$, nADCt $90 \%$ and $86.7 \%$, nADCe $80 \%$ and $90 \%$, rCBF $93.3 \%$ and $100 \%$, and rCBV $60 \%$ and $100 \%$. Combining Ev, Ev-Tv ratio, nADCt, nADCe and rCBV between MET and LGG gave $93.3 \%$ sensitivity and $100 \%$ specificity. Combining nADCt and rCBV between HGG and LGG $86.7 \%$ sensitivity and $100 \%$ specificity. 
Conclusion: Multi-parametric imaging protocols is an advantage for preoperative distinction of LGG, HGG and MET.

\section{1-P.56}

Category: Diagnostic Brain

Presentation Type : Poster Presentation

\section{Keywords :}

Volume perfusion CT imaging of cerebral vasospasm: diagnostic performance of different perfusion maps

Ahmed E. Othman ${ }^{1}$, Saif Afat ${ }^{2}$, Omid Nikoubashman ${ }^{2}$, Marguerite Müller ${ }^{2}$, Gerrit Alexander Schubert ${ }^{3}$, Georg Bier ${ }^{4}$, Marc A. Brockmann ${ }^{5}$, Martin Wiesmann ${ }^{2}$, Carolin Brockmann ${ }^{2}$

University Hospital Tuebingen Department of Diagnostic and Interventional Radiology Tuebingen-Germany ${ }^{1}$ University Hospital Aachen Department of Diagnostic and Interventional Neuroradiology Aachen-Germany ${ }^{2}$ University Hospital Aachen Department of Neurosurgery Aachen-Germany ${ }^{3}$ University Hospital Tuebingen Department of Neuroradiology Tuebingen-Germany ${ }^{4}$ University Hospital Mainz Department of Diagnostic and Interventional Neuroradiology Mainz-Germany ${ }^{5}$

Introduction: In this study, we aimed to evaluate the diagnostic performance of different volume perfusion CT (VPCT) maps regarding the detection of cerebral vasospasm compared to angiographic findings.

Methods: Forty-one datasets of 26 patients with subarachnoid hemorrhage and suspected cerebral vasospasm, who underwent VPCT and angiography within $6 \mathrm{~h}$, were included. Two neuroradiologists independently evaluated the presence and severity of vasospasm on perfusion maps on a 3-point Likert scale. A third neuroradiologist independently assessed angiography for the presence and severity of vasospasm on a 3-point Likert scale. Perfusion maps of cerebral blood volume (CBV), cerebral blood flow (CBF), mean transit time (MTT), and time to drain (TTD) were evaluated regarding diagnostic accuracy for cerebral vasospasm with angiography as reference standard. Correlation analysis of vasospasm severity on perfusion maps and angiographic images was performed. Furthermore, inter-reader agreement was assessed regarding findings on perfusion maps.

Results: Diagnostic accuracy for TTD and MTT was significantly higher than for all other perfusion maps $(\mathrm{TTD}, \mathrm{AUC}=0.832$; MTT, AUC $=$ $0.791 ; p<0.001)$. TTD revealed higher sensitivity than MTT $(p=0.007)$. The severity of vasospasm on TTD maps showed significantly higher correlation levels with angiography than all other perfusion maps ( $\mathrm{p} \leq$ 0.048 ). Inter-reader agreement was (almost) perfect for all perfusion maps (kappa $\geq 0.927$ ).

Conclusion: The results of this study indicate that TTD maps have the highest sensitivity for the detection of cerebral vasospasm and highest correlation with angiography regarding the severity of vasospasm.

\section{1-P.57}

Category: Diagnostic Brain

Presentation Type : Poster Presentation

\section{Keywords :}

Diagnostic performance of Low-Dose Volume Perfusion CT for the detection of cerebral vasospasm

Saif Afat $^{1}$, Carolin Brockmann ${ }^{1}$, Marguerite Müller ${ }^{1}$, Omid Nikoubashman ${ }^{1}$, Marc A. Brockmann ${ }^{2}$, Konstantin Nikolaou ${ }^{3}$, Martin Wiesmann ${ }^{1}$, Ahmed E. Othman ${ }^{3}$
University Hospital Aachen Department of Diagnostic and Interventional Neuroradiology Aachen-Germany ${ }^{1}$ University Hospital Mainz Department of Diagnostic and Interventional Neuroradiology MainzGermany ${ }^{2}$ University Hospital Tuebingen Department of Diagnostic and Interventional Radiology Tuebingen-Germany ${ }^{3}$

Purpose: In this study, we aimed to assess the diagnostic performance of Low-Dose Volume Perfusion CT (VPCT) for the detection of cerebral vasospasm as compared to angiography.

Method: 38 datasets from 25 patients with subarachnoid hemorrhage and suspected cerebral vasospasm were included. VPCT and angiography were performed within 6 hours. VPCT images were acquired at $80 \mathrm{kVp}$ and $180 \mathrm{mAs}$. Low-Dose VPCT datasets at $40 \%$ of the original dose levels were generated using a realistic low-dose Simulation method. Perfusion maps (CBF, CBV, MTT, TTD) were generated for both original and low-dose datasets. Two blinded neuroradiologists independently evaluated presence and severity of vasospasm on original and low-dose perfusion maps on a 3-point Likert scale. A third neuroradiologist independently assessed angiography for presence and severity of vasospasm on a 3-point Likert scale. Original and low-dose perfusion maps were evaluated regarding diagnostic accuracy for cerebral vasospasm with angiography as reference standard. Correlation analysis of findings on perfusion maps and on angiography was performed. Furthermore, the agreement between original and low dose images was assessed.

Results: Perfusion maps from original and low-dose datasets yielded comparable diagnostic accuracy without significant differences (Original: $\mathrm{AUC}=.818$; low-dose: $\mathrm{AUC}=. .815 ; \mathrm{p}=.906$ ). Findings on original and on low-dose perfusion maps showed strong correlation with angiography (original: $r=.664$; MS: $r=656$ ). Findings in original and low-dose perfusion maps showed almost perfect agreement (Kappa=.916).

Conclusion: Results of this study indicate that a radiation dose reduction of VPCT to $40 \%$ of the original radiation dose yields comparable diagnostic accuracy for the detection of cerebral vasospasm to standard dose.

\section{1-P.58}

Category: Diagnostic Brain

Presentation Type : Poster Presentation

Keywords :

The use of Vertebral artery Surface Anatomical Scanning (VSAS) in patients of vertebral artery dissection for anatomical diagnosis of association between vertebral artery and posterior inferior cerebellar artery

$\underline{\text { Kensaku Yoshida }}{ }_{-}^{\text {- }}$

Tokyo Metoropolitn Hiroo Hospital Neurosurgery Tokyo-Japan ${ }^{1}$

Purpose: Basiparallel anatomic scanning (BPAS)-MR imaging is useful to evaluate the surface appearance of the vertebrobasilar artery. However it is sometimes difficult for only BPAS-MR imaging to detect the transverse direction of the vertebral artery due to running from lateral to anterior surface of medulla oblongata. We try to evaluate the surface imaging along the transvers direction of the vertebral artery using BPAS-MR imaging technique. Materials and Methods: We obtained the surface imaging along the transvers direction of the vertebral artery, in addition to BPAS-MR imaging and 3D TOF MRA, for 25 consecutive patients who underwent brain MR imaging and MRA in our hospital between August 1 and December 31, 2015. Comparing Between BPAS-MR imaging and surface imaging of the transvers direction of vertebral artery, we evaluate the character and determined the role and value of displaying vascular outer contour.

Result: The surface imaging along the transvers direction was useful information to confirm the dissecting vertebral arteries and to reveal the whole appearance of the vertebral artery. This surface imaging had a 
possibility that observation of a vertebra artery became easy. However the oblique view has disadvantage, it is difficult to catch the anatomical structure around the vertebral artery.

Conclusion: The surface imaging along the transvers direction of the vertebral artery had a possibility that observation became easy and gave useful information.

\section{1-P.59 \\ Category: Diagnostic Brain \\ Presentation Type : Poster Presentation}

Reduction of Intracranial Clip Artifacts using Monoenergetic Extrapolations from Dual-Energy Computed Tomography

Sebastian Winklhofer ${ }^{1}$, Gerasimos Baltsavias ${ }^{1}$, Ricarda Hinzpeter ${ }^{2}$, JanKarl Burkhardt $^{3}$, Antonios Valavanis ${ }^{1}$, Hatem Alkadhi ${ }^{2}$

University Hospital Zurich, University of Zurich Department of Neuroradiology Zurich-Switzerland ${ }^{1}$ University Hospital Zurich, University of Zurich Institute of Diagnostic and Interventional Radiology Zurich-Switzerland ${ }^{2}$ University Hospital Zurich, University of Zurich Department of Neurosurgery Zurich-Switzerland ${ }^{3}$

Purpose: To assess the value of monoenergetic extrapolations from dual-energy computed tomography (DECT) for metal artifact reduction of intracranial clips of various materials and sizes. Materials and Methods: In this experimental phantom study, artifacts of six titanium clips (straight shape: size mini, small, medium and large; curved shape: size small and medium) and three Phynox (CobaltChromium-Nickel alloy) clips (straight shape: size small and medium; curved shape: size medium) were examined using DECT scans. Postprocessing included routinely used standard $80 / 150 \mathrm{kVp}$ mixed energy images and additional postprocessed monoenergetic extrapolations with $120 \mathrm{keV}$. Image analysis included the quantitative assessment of image artifacts (Hounsfield units [HU] measurements) as well as the qualitative evaluation of artifact severity and the visualization of contrast media filled adjacent simulated vessels using a four-point Likert scale. Results: HU values of streak artifacts were significantly $(\mathrm{P}<0.05)$ different between standard 80/150 kVp images (mean $-228 \pm 199 \mathrm{HU}$ ) and monoenergetic reconstructions $(-2.1 \pm 13.1 \mathrm{HU})$. Artifact scores improved significantly from $80 / 150 \mathrm{kVp}$ images (median 3 ) to monoenergetic extrapolations (median $1 ; \mathrm{P}<0.05$ ). Visualization scores of simulated vessels were significantly $(\mathrm{P}<0.05)$ better with monoenergetic extrapolations (median 1) compared to $80 / 150 \mathrm{kVp}$ images (median 2).

Conclusions: Monoenergetic extrapolations from DECT allow for a reduction of artifacts and a better assessment of clips and adjacent vessels compared to standard image reconstructions.

\section{1-P.60}

Category: Diagnostic Brain

Presentation Type : Poster Presentation

Keywords : Stroke, Microbleeds, Gradient-Echo sequences, SWI sequences

The Efficiency Of Gradient Echo (GRE) And Susceptibility Weighted Imaging (SWI) Sequences In The Detection Of Early Cerebral Parenchymal Microbleeds In Patients With Acute Stroke

Zafer Sarıaltın ${ }^{1}$, Kamil Karaali ${ }_{-}^{1}$, Ali Ünal ${ }^{2}$, Utku Șenol ${ }^{1}$

Akdeniz University Radiology Antalya-Turkey ${ }^{1}$ Akdeniz University Neurology Antalya-Turkey ${ }^{2}$
In this study, efficiency of gradient echo (GRE) and susceptibility weighted imaging (SWI) sequences in the detection of cerebral parenchymal microbleeds in acute stroke patients who referred to our MRI unit were evaluated.

Between November 2010 - January 2012, all patients who were referred to our MRI unit with acute stroke were included, if both GRE and SWI sequences were obtained along with routine brain MRI sequences and if they were both in good diagnostic quality. Fifty-four patients (34 male, 20 female) were included. Ages ranged between 37-95 years (mean 70 years). All sequences were evaluated by 2 specialists with experience more than 10 years in neuroradiology. Firstly, GRE sequneces and a week later, SWI sequences were evaluated. Efficiency of the sequences in the detection was evaluated by comparing the number of foci. The difference between the sequences was evaluated by Mc Nemar test and agreement was evaluated by Cohen's Kappa test.

According to Cohen's kappa test, agreement between the methods was found to be fair. After consensus evaluation, agreement between the sequences was also found to be fair (k: 0,23$)$. McNemar test showed that the $\mathrm{p}$ value is 0,04 and the methods are different and can not be used interchangeably. The number of detected microbleeds within the infarction area or outside the infarction was more in SWI sequence than GRE sequence. As the new sequences that are sensitive to susceptibility are getting developed, the microbleeds are detected much more. However, the effect of this finding on the therapeutic approach is still controversial.

\section{1-P.61}

Category: Diagnostic Brain

Presentation Type : Poster Presentation

Keywords : flat-detector CT, flat-detector CT Angiography, neuroimaging, metal artifact reduction algorithm

Evaluation of an optimized metal-artifact-reduction algorithm for flatdetector CT angiography

Nadine Amelung ${ }^{1}$, Daniel Behme ${ }^{1}$, Ioannis Tsogkas ${ }^{1}$, Michael Knauth ${ }^{1}$, Marios Nikos Psychogios ${ }^{1}$

University Clinic Göttingen Neuroradiology Göttingen-Germany ${ }^{1}$

Introduction: Flat-detector CT (FDCT) has become a valuable tool in periinterventional imaging of neurointerventional procedures and neurosurgical follow up. Metal induced artifacts usually impair imaging analysis. Recent optimization in metal-artifact-reduction algorithms (MARA) improved imaging analysis.

Methods: In this study follow up examinations after aneurysm therapy either by coiling or by clipping were conducted via FDCT-Angiography (FDCTA. Prior examinations via DSA were available for comparison. FDCTA-data sets were processed by a MARA-prototype. Images were randomized and evaluated by one rater, as well as the DSA data. The rater was blinded for application of the algorithm and evaluated the images concerning the extent of the metal artifacts on a scale from 1 to 4 ( 1 no to 4 - extensive artifacts). He also rated the images concerning particular questions.

Results: Correct identification of implemented metal devices and parent vessels was always possible. The extent of artifacts was rated less severe in 7 of 10 cases when comparing MARA-manipulated datasets and the original datasets. In 3 cases image quality was rated equal. Concerning evaluation of a residual perfusion of the treated aneurysm MARAoptimized images displayed a repercussion in two cases, corresponding to DSA, where original data could not be evaluated. In 2 other cases results of MARA-optimized images and DSA were equal, where original data showed a contrary result.

Conclusion: Application of MARA-prototype leads to a significant reduction of metal artifacts, improving the evaluation of clipped or coiled aneurysms. In 2 of 10 cases reperfusion could be diagnosed or excluded only after application of MARA. 
1-P.62

Category: Diagnostic Brain

Presentation Type : Poster Presentation

Keywords : Headache, pediatric patient, MRI.

\section{MRI Findings In Pediatric Patients Presenting With Headache}

Aydın İnce ${ }^{1}$, Ali Dablan ${ }^{1}$, Öznur Bozkurt ${ }^{2}$, Özgür Duman ${ }^{2}$, Kamil Karaali ${ }^{1}$, Hakk1 Timur Sindel ${ }^{1}$

Akdeniz Universty Radiology Antalya-Turkey ${ }^{1}$ Akdeniz Universty Pediatric Neurology Antalya-Turkey ${ }^{2}$

Purpose: In this study, we aimed to evaluate pathologies detected by MRI in pediatric patients applying with isolated headache.

Materials and Methods: MRIs of pediatric patients referred to our department between 2013-2014 with isolated headache and no systemic or neurological symptoms apart from aura were retrospectively evaluated. Out of 447 patients included in the study, $271(60,6 \%)$ were female and $176(39,4 \%)$ were male. Their age varied between 3-1.

Results: 158 pathologies $(29,5 \%)$ were detected in 132 patiens. 54 $(34,2 \%)$ white matter lesions, $3(1,9 \%)$ cortical pathologies, 3 $(1,9 \%)$ basal ganglia lesions, $2(1,3 \%)$ brainstem lesion, $1(0,6 \%)$ cerebellar pathology, $1(0,6 \%)$ malformation, $4(2,5 \%)$ vascular malformations, $2(1,3 \%)$ other vascular pathologies, $53(33,5 \%)$ sinusitis, $8(5,1 \%)$ mastoid inflammation, $7(4,4 \%)$ arachnoid cysts, 10 $(6,3 \%)$ other cysts and mass lesions, $2(1,3 \%)$ ischemic lesions, 6 $(3,8 \%)$ variations and $2(1,3 \%)$ ventricular pathologies were detected. There was no difference in age and sex between patients with and without lesions. White matter lesions were more common in children older than 10 whereas sinusitis was more common in children younger than $10(p=0,019$ and 0,029 respectively). For both pathologies there was no significant difference in sex $p=0,708$ and 0,968 respectively).

Conclusion: MRI demonstrates findings that require treatment or change treatment plan in only a very small number of patients applying with isolated headache.

\section{1-P.63}

Category: Diagnostic Brain

Presentation Type : Poster Presentation

Keywords : MRI, stroke mimic, migraine aura

\section{Morphological and perfusion MRI abnormalities during acute mi- graine aura}

Gilles Adam $^{1}$, Alain Viguier ${ }^{2}$, Vanessa Cazzola ${ }^{1}$, Marine Ferrier ${ }^{1}$, Manuela Kamsu 1, Sofia Patsoura ${ }^{1}$, Raluca Gramada ${ }^{1}$, Christophe Cognard ${ }^{1}$, Nelly Fabre ${ }^{2}$, Fabrice Bonneville ${ }^{1}$

University hospital of Purpan Diagnostic and therapeutic neuroradiology service Toulouse-France ${ }^{1}$ University hospital of Purpan Neurology service Toulouse-France ${ }^{2}$

Introduction: Acute migraine aura may mimic acute ischemic stroke. The aim of this study was to describe the morphological and perfusion MRI abnormalities observed during acute migraine aura.

Method: Retrospective study of 14 patients (mean age: 23,6 years) explored by MRI during acute neurological deficit related to a migraine aura. Patients presented with aphasia (13/14), sensory (7/14) or visual disturbances (8/14). Diagnosis of migraine was confirmed by neurologists. We here analyzed: signal intensity of the brain parenchyma, visibility of the cerebral arteries and of the cortical veins on $\mathrm{T} 2 *$, and variation of classic perfusion parameters.

Results: MRI demonstrated abnormalities on the contralateral side of the neurological deficit in all the cases. There was no abnormal signal intensity of the brain parenchyma. Left middle and/or posterior cerebral arteries were less visible distally in $8 / 14$ patients. Areas of cerebral hypoperfusion not limited to a single arterial territory were present in $14 / 14$ patients. They appeared as left parietal-temporal-occipital hypoperfusion, sometimes extended to right parietal-temporal-occipital lobes $(1 / 11)$ or to the left frontal lobe (6/11), with elongated TTP (14/14), subtle decreased rCBF and rCBV (11/14). Crossed cerebellar diaschisis with elongated TTP was detected in 7/14 patients. Facing areas of cerebral hypoperfusion, cortical veins were abnormally visible on $\mathrm{T} 2 *$ in all the cases.

Conclusion: During migraine aura, MRI demonstrates asymmetrical cerebral hypoperfusion with left posterior predilection not limited to a single arterial territory, and associated crossed cerebellar diaschisis. Additional fading of distal cerebral arteries and enhanced visibility of cortical veins are facing areas of cerebral hypoperfusion.

\section{1-P.64}

Category: Diagnostic Brain

Presentation Type : Poster Presentation

Keywords : action execution, action observation, motor imagery, mirror neuron theory, mental simulation theory

\section{Neural foundations of goal directed overt and covert actions}

Efrosini Papadaki ${ }^{1}$, Eleftherios Kavroulakis ${ }^{1}$, Georgios Kalaitzakis ${ }^{2}$, Themistoklis Boursianis ${ }^{2}$, Thomas Maris ${ }^{2}$, Helen Savaki ${ }^{3}$, Panagiotis Simos ${ }^{4}$

University of Crete Radiology Heraklion-Greece ${ }^{1}$ University of Crete Medical Physics Heraklion-Greece ${ }^{2}$ University of Crete Neuroscience Heraklion-Greece ${ }^{3}$ University of Crete Psychiatry Heraklion-Greece ${ }^{4}$

Purpose: To assess by fMRI: (i) the human brain areas involved in the execution, observation and imagery of a tracing action with the right index finger, (ii) possible distinction between the overt action and its covert counterparts.

Methods and Materials: The fMRI block design included three tasks (Execution, Imagery, Observation) each consisting of four 35-sec blocks was performed to 13 volunteers.

Results: (i) voxel-level analysis revealed a common circuit activated across all three conditions comprising primary motor and somatosensory, dorsal and ventral premotor, superior and inferior parietal, posterior middle and superior temporal as well as superior occipital cortical areas. (ii) Activation of the anterior cerebellum and the anterior BA7-precuneus exclusively for action execution as well as activation of the extrastriate body area and BA9 exclusively for action observation may contribute to the distinction between the "self" and the "other". Also, the preSMA activated exclusively for motor imagery may contribute to the differentiation between "real" and "imagined" actions. The more extensive and intense activation of the primary motor cortex for overt than covert actions, as well as the parietal activation which was more extended for imagery and more intense for observation, compared to execution, may also contribute to the distinction between overt and covert actions.

Conclusion: Both motor imagery and observation of a goal directed action involve sensory-motor brain circuits largely overlapping with those supporting execution of the same action. 
This is in accordance with the Mental Simulation Theory and against the Mirror Neuron Theory.

\author{
1-P.65 \\ Category: Diagnostic Brain \\ Presentation Type : Poster Presentation
}

Keywords : MRI, ASL, Epilepsy, EEG, Epileptic Zone

\section{Arterial Spin Labeling Perfusion in Pediatric Epilepsy. Experience in the Institute of Neurosurgery dr. Asenjo.}

Francisco Torres ${ }^{1}$, Cecilia Okuma ${ }^{1}$, Francisca Montoya ${ }^{1}$, Marcelo Jara , Mario Castro ${ }^{-}$, Aaron Vidal ${ }^{1}$, Roberto Marileo ${ }^{1}$, Ingeborg López ${ }^{1}$, Daniel Echeverria ${ }^{1}$, Lautaro Badilla ${ }^{1}$

Institute of Neurosurgery Dr. Asenjo Neuroradiology Santiago-Chile ${ }^{1}$

Background: Arterial Spin Labeling (ASL) perfusion is a MRI technique to quantitatively measuring cerebral blood flow, that labels the protons from the arterial water as a freely diffusible tracer. ASL is noninvasive and performed without contrast media. Technical advances have increased its sensitivity extending its potential applications. In epilepsy given the correlation between ASL and PET with electrophysiology, ASL might help to confirm the location of the epileptogenic zone (EZ).

Methods: We report the initial experience in pediatric epilepsy of the ASL in the Institute of Neurosurgery Dr. Asenjo. MRI was conducted on a Siemens 3.0 T MAGNETOM Skyra head scanner (Siemens, Erlangen, Germany). The patients underwent an assessment with MR acquisition protocol including structural imaging and pulsed ASL, and the inter ictal electroencephalography (EEG) or video-EEG.

Results: We evaluated 48 pediatric patients with epilepsy or a convulsive syndrome, between August 2015 and June 2016. 25 (52\%) men; mean age 7,65 (1 month to 16 year). $27(56,25 \%)$ had lesions on structural MRI. ASL hypoperfusion was seen in $33(68,7 \%)$ patients and artifacts in the ASL were found in $7(14,6 \%)$. The EEG was abnormal in $9(22 \%)$. The concordance between ASL and EEG was seen in $17(54,8 \%)$. We find a contralateral EZ in $5(16,12 \%)$ and another focus ipsilateral or multiples focus in $7(22,58 \%)$. Conclusions: The correlation between ASL and EEG might help to evaluate the EZ and the find of a contralateral focus could be in the context of a mirror focus

\section{1-P.66}

Category: Diagnostic Brain

Presentation Type : Poster Presentation

Keywords : Glioblastoma, Metastasis, Susceptibility-Weighted Imaging, Apparent Diffusion Coefficient

Necrotic Glioblastomas and Necrotic Metastatic Brain Tumors: Discrimination with Susceptibility-Weighted Imaging and the Apparent Diffusion Coefficient

Ping-Hong Lai ${ }^{1}$, Tzu-Chao Chuang ${ }^{2}$, Jui-Hsun Fu ${ }^{1}$, Po-Chin Wang ${ }^{1}$, Shuo-Hsiu Hsu ${ }^{\mathrm{T}}$, Huey-Shyan Lin ${ }^{3}$

Kaohsiung Veterans General Hospital Radiology Kaohsiung-Taiwan ${ }^{1}$ National Sun Yat-Sen University Electrical Engineering KaohsiungTaiwan ${ }^{2}$ School of Nursing, Fooyin University Program of HealthBusiness Administration Kaohsiung-Taiwan ${ }^{3}$

Purpose: Necrotic glioblastomas multiforme can simulate intracranial necrotic metastasis in MRI appearance. The purpose of this study was to compare findings of Susceptibility-Weighted Imaging (SWI) with those of the apparent diffusion coefficient (ADC) for this differential diagnosis.
Methods: SWI was performed in 31 patients with rim-enhancing glioblastomas and 21 patients with rim-enhancing metastases at 1.5 Tesla. Two observers evaluated the degree of intralesional susceptibility signal (ILSS) seen on SWI. Chi-square test was used to assess the association between rim-enhancing lesion and the degree of ILSS. Average ADC was calculated in the cystic cavity. To analyze ADC between rim-enhancing lesions, ANOVA was performed. After receiver operating characteristic (ROC) analysis, area under ROC curve (AUC) was compared between SWI and ADC.

Results: The grade of ILSSs was significantly different between rimenhancing lesions in the differentiation of glioblastoma from metastasis $(\mathrm{P}=.009)$. When distinguishing glioblastoma from metastasis, the percentage of ILSS grade 3 was $61.3 \%$ in glioblastoma and higher than metastasis (28.6\%). There was no significant difference of the ADC value between glioblastoma and metastasis. For distinguishing glioblastoma from metastasis, AUCs of SWI and ADC were 0.73 and 0.51 , respectively. SWI was significantly better than $\mathrm{ADC}(\mathrm{P}=.039)$.

Conclusions: ILSS was useful for differentiating between necrotic glioblastomas and necrotic metastatic brain tumors. A high-grade ILSS may help distinguish glioblastomas from solitary metastatic brain tumors. ADC was limited in differentiating between necrotic glioblastomas and necrotic metastatic brain tumors.

\section{1-P.67}

Category: Diagnostic Brain

Presentation Type : Poster Presentation

\section{Keywords :}

Diffusion tensor imaging shows brain structural damage associated with verbal memory in subjects with multiple sclerosis

Gerard Blasco ${ }^{1}$, Briggith Beltrán ${ }^{1}$, Carles Biarnés ${ }^{1}$, Jordi Gich ${ }^{2}$, Mireia Rivero $^{2}$, Judith Salvedra ${ }^{2}$, Pepus Daunis-i-Estadella ${ }^{3}$, Salvador Pedraza ${ }^{1}$, Lluís Lamió $^{2}$, Josep Puig ${ }^{1}$

Imaging Research Unit. Diagnostic Imaging Institiute (IDI). Dr. Josep Trueta University Hospital Radiology Girona-Spain ${ }^{1}$ Neuroimmunology and Multiple Sclerosis Unit, Department of Neurology, Dr. Josep Trueta University Hospital Neurology Girona-Spain ${ }^{2}$ University of Girona Department of Computer Science, Applied Mathematics and Statistics Girona-Spain ${ }^{3}$

Purpose: We studied differences in diffusion tensor imaging metrics (DTI-metrics) of white matter structural integrity among cohorts of subjects with multiple sclerosis (MS) classified according to progression and their relation to verbal memory.

Methods: We prospectively studied consecutive patients with radiologically or clinically isolated syndrome $(n=23)$ primary or secondary progressive (PP/PS) MS ( $n=35)$, relapsing-remitting (RR) $(n=24)$ MS, and 30 healthy controls on a $1.5 \mathrm{~T}$ scanner. Imaging included DTI and anatomic T1 TFE. We used Olea Sphere 3.0 to map axial diffusivity (AD), radial diffusivity (RD), mean diffusivity, and fractional anisotropy (FA); and Juelich's Atlas to identify white matter tracts. We estimated verbal memory by the Long Term Storage (LTS) Selective Reminding Test (SRT) subtests. We used analysis of variance to assess differences in SRT among groups and analysis of covariance (ANCOVA) including variables associated with cognitive scores $(p<0.01)$ to analyze relations between DTI-metrics and SRT among groups.

Results: Groups differed on SRT-LTS $(\mathrm{p}<0.001)$. The best ANCOVA model to explain SRT-LTS scores among groups $(\mathrm{p}=0.002)$ included right acoustic radiation $\mathrm{AD}(\mathrm{p}=0.001)$, corpus callosum $\mathrm{AD}(\mathrm{p}=0.004)$ and $\mathrm{RD}$ $(p=0.005)$, left corticospinal tract $A D$ and $R D$ (both $p=0.001)$, right corticospinal tract $\mathrm{RD}(\mathrm{p}=0.006)$, left fornix FA $(\mathrm{p}=0.004)$, right optical 
radiation $\mathrm{RD}(\mathrm{p}=0.028)$, right superior longitudinal fascicle $\mathrm{RD}$ $(\mathrm{p}=0.006)$, and total grey matter volume $(\mathrm{p}=0.006)$.

Conclusion: Our preliminary results suggest structural white matter integrity measured by DTI is associated with verbal memory scores in MS according to progression.

\section{1-P.68}

Category: Diagnostic Brain

Presentation Type : Poster Presentation

Keywords : Parkinson's disease, Nigrosome-1, QSM, SWI, Susceptibility

Multiple Susceptibility based MRI Techniques for the Diagnosis of Nigrosome1

$\underline{\text { Kyung Mi Lee }}{ }_{-}{ }_{-}$, Hyug-gi Kim ${ }^{1}$, Eui Jong $\mathrm{Kim}^{1}$, Woo Suk Choi Choi ${ }^{1}$

Kyung Hee University Hospital Radiology Seoul-Korea, South ${ }^{1}$

Introduction: To investigate the usefulness of susceptibility-based MR imaging techniques for classification normal subjects with parkinson's disease(PD) patients by visualization of nigrosome-1.

Material and Method: 23 PD patients and 18 normal subjects underwent 3D multiple-TE FFE sequence. 3T MR images were acquired in each subject with a 32-channel SENSE head coil. 3D FFE sequence was run with 7 echoes to obtain magnitude and phase image: first echo time(TE)/ $\triangle \mathrm{TE}=3.38 / 6.12$. For magnitude data, R2* and averaged magnitude image(MAGavg) was performed in Matlab. To generate QSM, acquired magnitude and phase images were processed by implementing the MEDI method.

All subjects were organized as groups according to the results of susceptibility based MR images. To evaluate between normal subject and PD patient, the quantitative susceptibility value of nigrosome-1 was measured by using drawing ROIs, respectively.

Results: 3 Groups were organized for the degree of visualizing nigosome1: group I was the equivocal bad, group II was the equivocal good subjects for three susceptibility based MRI techniques and group III was the best visualizing subjects in QSM map compared to R2* map and SWI.

Fig. 1 demonstrates comparisons for visualizing nigrosome-1 of R2* map, SWI and QSM map obtained from multiple echoes based FFE sequence for a subject in group III.

Conclusion: QSM-MRI technique proved to be more effective to evaluate the early stage for PD than R2* map or SWI. Furthermore, the QSM technique can be used as an imaging biomarker to evaluate PD.

\section{2 - Interventional}

\section{2-P.01}

Category: Interventional

Presentation Type : Poster Presentation

Keywords : intracranial aneurysm genetic biocollection familial physiopathology

\section{The ICAN project : Understanding the pathophysiology of intracra- nial aneurysm}

Romain Bourcier $^{{ }^{1}}{ }^{\text {, }}$ Cedric Lenoble ${ }^{1}$, Benjamin Daumas-Duport ${ }^{1}$, Vincent l'allinec ${ }^{1}$, Alexis Moles ${ }^{1}$, Hubert Desal ${ }^{1}$

CHU de Nantes Neuroradiology Nantes-France ${ }^{1}$

Although the pathogenesis of intracranial aneurysm (IA) has been the subject of many studies for the last decade, the mechanisms underlying IA formation, growth and rupture are still mostly unknown and relevant animal models of IA are not available. Familial history of IA predisposes to IA formation and rupture and increasing evidence suggest a genetic component of IA formation, with heterogeneous modes of inheritance and penetrance.

Our project, gathering neuroradiologists, geneticists and vascular biologists, addresses the urgent need to understand the pathogenic mechanisms of IA to develop diagnostic and predictive tools of risk of IA. Neuroradiological phenotyping of the IA is done by experienced interventional neuroradiologists of each center, and inclusion and exclusion criteria have been carefully set up to ensure a precise phenotypic characterization and to only include typical saccular IA of bifurcations. We propose to identify IA-causing variants by whole-exome sequencing in familial forms of the disease. We hypothesize that the functional analysis of the causal/susceptibility variants thus identified will provide clues to understanding the pathological mechanisms of IA formation, and the bases for developing diagnostic tools. Based on our preliminary data that already allowed to identify such a variant, we are know building a nationwide multicentric network to recruit sporadic and familial IA. This large biocollection including clinical, biological, genetic and imaging data will offers a unique opportunity to put in relation genetic variation and accurate phenotyping in this orphelin pathology.

2-P.02

Category: Interventional

Presentation Type : Poster Presentation

Keywords : Endovascular embolization, Dural Arteriovenous fistula

Single center experience with Endovascular Treatment of Intracranial Dural Arteriovenous Fistulas

Andrijana Jovic $^{1}{ }_{-}$, Vladimir Kalousek ${ }^{2}$, Branimir Čulo ${ }^{1}$, Krešimir Rotim $^{3}$

UHC "Sisters of Mercy" Department of diagnostic and interventional radiology Zagreb-Croatia "UHC" Sisters of Mercy" Neurointerventional Unit Zagreb-Croatia ${ }^{2}$ UHC" Sisters of Mercy" Department of Neurosurgery Zagreb-Croatia ${ }^{3}$

Single center experience with Endovascular Treatment of Intracranial Dural Arteriovenous Fistulas

Aims and Objectives: Dural arteriovenous fistulas (DAVFs) represents the most dangerous type of intracranial AV shunt. Endovascular treatment(EVT) is first option for management of these lesion. Our purpose is to bring our experience with EVT of DAVFs.

Materials and Methods: Since December 2013 until December 2015, 8 patient underwent EVT of DAVF. Recorded baseline data were sex, age, data of earlier head trauma, clinical status before and after the procedure, angioarhitecture of DAVF-venous drainage pattern by Cognard. For each procedure were recorded procedure details, complications and result of the procedure. Control MRI was performed three and six months after the procedure.

Results: There were 5 men and 3 women, age ranging from 39 to 72 (mean, $62.4 \pm 10.4$ years). None case had previously head trauma. One case had DAVF Cognard type IIb, three cases had type III, and four cases had type IV. In 4 cases posttreatment result was complete obliteration of DAVF. Reduction of DAVF was result in other 4 cases. In 7 cases clinical status was much improved after the treatment. One case died few days after the treatment as result of earlier intracranial hemorrhage. Three months after the procedure control MRI showed complete obliteration of the fistula at four cases and reduction of DAVF at three cases. Control MRI six months after the procedure showed complete obliteration of DAVF at another two cases.

Conclusion: Endovascular embolization in our experience is high effective therapy in the treatment of DAVF. 
2-P.03

Category: Interventional

Presentation Type : Poster Presentation

Keywords : Stroke, Mechanical Thrombectomy, Endovascular Procedures, Thromboaspiration

Single-Centre Experience Using the 3MAX Reperfusion Catheter for the Treatment of Acute Ischemic Stroke with Distal Arterial Occlusion

Kévin Premat ${ }^{1}$, Bruno Bartolini ${ }^{1}$, Flore Barronnet ${ }^{2}$, Nader Sourour ${ }^{1}$, Joseph Gabrieli ${ }^{1}$, Charlotte Rosso ${ }^{2}$, Federico Di Maria ${ }^{1}$, Silvia Pistocchi ${ }^{1}$, Vincent Degos ${ }^{3}$, Yves Samson ${ }^{2}$, Jacques Chiras ${ }^{1}$, Frédéric Clarençon ${ }^{1}$

Pitié-Salpêtrière Hospital Interventional Neuroradiology 75013-France ${ }^{1}$ Pitié-Salpêtrière Hospital Vascular Neurology 75013-France ${ }^{2}$ PitiéSalpêtrière Hospital Anaesthesiology 75013-France ${ }^{3}$

Background and Purpose: Most recent guidelines recommend the use of stent retriever devices in endovascular treatment of acute ischemic stroke with large vessel occlusion. Recently published data reported promising results with tromboaspiration devices such as the Penumbra System (Penumbra, Alameda, California) combined with the Penumbra MAX series reperfusion catheters by using the ADAPT technique. The aim of this study was to report our initial experience with the $3 \mathrm{MAX}(3.8 \mathrm{~F})$ reperfusion catheter for the recanalization of distal intracranial arteries. Methods: From August 2015 to January 2016, 12 consecutive patients (7 women $[58.3 \%]$ and 5 men $[41.7 \%]$; mean age $=68.1 \pm 17.6 \mathrm{y}$, [range: 38 91]) for 15 occluded arteries underwent mechanical thrombectomy (MT) by thromboaspiration using the Penumbra $3 \mathrm{MAX}$ catheter. The primary endpoint of the study was the Thrombolysis In Cerebral Infarction (TICI) score after thromboaspiration attempts alone using the 3MAX reperfusion catheter. Major and minor procedure-related complications were also systematically recorded

Results: In 1/15 (6.7\%) case, the 3MAX could not be navigated into the target artery. Six out of the $14(42.9 \%)$ remaining arteries were successfully recanalized (TICI 2b/3) after thromboaspiration with the Penumbra $3 \mathrm{MAX}$ reperfusion catheter alone. The overall recanalization rate was $71.4 \%$. Three $(25 \%)$ procedure-related complications occurred, including two (16.7\%) 3MAX-related complications (one clot migration and one vessel perforation). Overall mortality rate was $16.7 \%$. No procedurerelated mortality was recorded.

Conclusion: The 3MAX reperfusion catheter is a safe device and provides interesting results in terms of distal occlusions' recanalization or for M1 recanalization in cases of narrowed ICA

\section{2-P.04 \\ Category: Interventional \\ Presentation Type : Poster Presentation}

Endovascular treatment of complex saccular and fusiform intracranial aneurysms with flow diverter devices

$\underline{\text { Tomaz Seruga }}{ }^{1}$, Marko Jevsek $^{1}$

University Clinical Centre Radiology Maribor-Slovenia ${ }^{1}$

Flow diversion represents the latest approach in endovascular treatment of complex, saccular and fusifiom intracranial aneurysms. The higher pore density of the device wall keeps the main blood stream in the lumen of the stent and slows down the turbulent flow in the aneurysm. Stagnation of blood in the aneurysm promotes the formation of thrombus and subsequent exclusion of the aneurysm from the patent artery.

Between November 2010 and February 2014 we treated 18 patients with a incidentally detected, unruptured aneurysm of the internal carotid artery and M1 segment of middle cerebral artery. The majority of aneurysms of the internal carotid artery were located at the ophthalmic part of the artery. The patients were treated initially using a Pipeline and recently with FRED. Treatment success was assessed clinicaly and angiographically using O'Kelly Marotta scale.

Control angiograms after the stent deployment showed signs of early stagnation of the blood flow in the aneurysms. All the procedures were performed without any technical or clinical complications. In two cases the FD devices were not fully expanded, so post dilatation was performed. Six months after the procedure, control MR and digital subtractional angiography showed in almost all cases exclusion of the aneurysm from the circulation and normal blood flow in the distal part of treated artery. Neurological status six months after the procedure was normal or improwed in all patients.

Treatment of intracranial aneurysms with flow diverter devices is a safe and significantly time sparring method.

\section{2-P.05}

Category: Interventional

Presentation Type : Poster Presentation

The dual lumen balloon catheter - a multipurpose device with unique features

Ljubisa Borota $^{1}$, Ehab Mahmoud ${ }^{1}$, Christoffer Nyberg ${ }^{1}$, Per Enblad ${ }^{2}$, Elisabeth Ronne-Engström ${ }^{2}$

University Hospital Centre for Medical Imaging Uppsala-Sweden ${ }^{1}$ University Hospital Department of Neurosurgery Uppsala-Sweden ${ }^{2}$

Aim of the Study: To describe our experience in the treatment of various pathological conditions of the cranial and spinal blood vessels and hipervascularized lesions using dual lumen balloon catheters.

Material and Methods: In total 17 patient, seven females and ten males, were treated by endovascular means for following conditions: spasm of cerebral blood vessels caused by subarachnoid hemorrhage, 1, hypervascularized metastasis in vertebral body, 1, spinal dural fistula, 1, cerebral dural fistula 2, cerebral AVM, 3 and aneurysms, 9. The dual lumen balloon catheter was used for remodeling of coil mesh, injection of various liquid embolic agents, particles and nimodipine, prevention of reflux and for deployment of coils and stents.

Results: Two complications were recorded: perforation of an aneurysm in one case and gluing of the tip of balloon catheter by embolic material in another case. All other interventions were uneventful and therapeutic goals were achieved in all cases except in the case with gluing of the tip of balloon catheter. Conclusions: The dual lumen balloon catheter is one of the most promising neurointerventional devices which contributes significantly to expansion of indication area as well as to increased safety of neurointerventional procedures. However, the special attention should be paid during the manipulation with these devices in order to avoid potentially severe complications.

2-P.06

Category: Interventional

Presentation Type : Poster Presentation

Keywords : VERTEBROPLASTY CERVICAL SPINE MALIGNANT LESIONS

VERTEBROPLASTY FOR CERVICAL LESIONS How to improve quality of filling and safety. 25 cases

Chiras Jacques ${ }^{1}$, Cormier Evelyne ${ }^{1}$, Clarencon Frederic ${ }^{1}$

Aphp 75 Paris-France ${ }^{1}$ 
Purpose: We present an original technique that reduce the risk of leakage in difficult vertebroplasties. Conventionnal technique of vertebroplasty is a comprimize between optimized filling and risk of leakage with clinical incidence as at the end of filling it is necessary to wash out the dead space of the needle $(1 \mathrm{ml})$. Injection of cement through Bone filler eliminates the dead space problem and authorize a more precise filling of the lesion.

Material and Method: 27 patients with cervical lytic lesion (24 metastases, 2 myelomas, 1 aggressive angioma) were treated by vertebroplasty using bone filler technique (41vertebrae).

Results: Adequate filling of the vertebra was obtained in $85 \%$ of cases. No clinical complication occurred even in cases with posterior wall disruption or fracture. In the population.All patients were discharged from the hospital at 24 H.Clinical improvement at 1 month follow-up was very high.

Conclusion: Vertebroplasty with bone filler injection should be recommended in difficult cases of vertebroplasty orcementoplasty with high risk of dangerous leakage

\section{2-P.07}

Category: Interventional

Presentation Type : Poster Presentation

\section{Keywords :}

Vascular angular remodeling by kissing-Y stenting in wide-necked intracranial bifurcation aneurysms

Katharina Melber ${ }^{1}$, Friedhelm Brassel ${ }^{1}$, Dan Meila ${ }^{1}$, Martin SchlunzHendann ${ }^{1}$, Dominik Grieb

Sana Kliniken Duisburg Department of Radiology and Neuroradiology Duirburg-Germany ${ }^{1}$

Background: Single stent deployment leads to a change of vascular bifurcation geometry in wide-necked bifurcation aneurysms. In some complex cases the use of the single stent technique might not be sufficient or not feasible. The kissing-Y stenting technique appears to be one alternative endovascular treatment option. The aim of this study was to evaluate the effects of the kissing-Y stenting technique on vascular angular remodeling.

Methods: A total number of 21 patients (13 female, 8 male) with wide-necked intracranial bifurcation aneurysms at different sites (9 AcomA, $6 \mathrm{MCA}, 4 \mathrm{BA}$, $1 \mathrm{VA} / \mathrm{PICA}, 1 \mathrm{ACI} / \mathrm{PcomA}$ ) were treated with 44 closed-cell stents (followup: 2012 - 2016) using the kissing-Y stenting technique. We analyzed vascular angle geometry between the mother and both affected daughter $(\delta 1, \delta 2)$ vessels by MRA and DSA before and after stent deployment using standardized working projections.

Results: The stents were successfully deployed in all cases. Endovascular treatment of wide-necked intracranial aneurysms using the kissing-Y stenting technique significantly straightened and increased both affected vascular bifurcation angles $(\mathrm{P}<0.01)$.

Conclusions: Kissing-Y stenting in wide-necked bifurcation aneurysms leads to vascular angular remodeling of both affected branches. The straightening of both bifurcation angles may narrow the flow impingement zone and thus, reduce the wall shear stress at the bifurcation apex.

\section{2-P.08}

Category: Interventional

Presentation Type : Poster Presentation

Treatment of a giant cavernous carotid artery aneurysm with two balloon-expandable stent grafts - a technical note

Dominik Grieb ${ }^{1}$, Dan Meila ${ }^{1}$, Katharina Melber ${ }^{1}$, Björn Greling ${ }^{1}$, Martin Schlunz-Hendann ${ }^{1}$, Friedhelm Brassel $^{\text {- }}$
Sana Kliniken Duisburg Department of Radiology and Neuroradiology Duisburg-Germany ${ }^{1}$

A 53-year-old woman presented to our hospital with diplopia. CCT and CTA revealed a giant, wide-necked aneurysm of the left cavernous ICA $(1.8 \mathrm{~cm} \times 1.6 \mathrm{~cm})$. The space-occupying effect of the aneurysm caused VIth nerve palsy. The aneurysm was treated by using two balloonexpandable coronary stent grafts (BeGraft Coronary, Bentley InnoMed, $4 \times 12 \mathrm{~mm}$ and $5 \times 16 \mathrm{~mm}, 0.014$ "-compatible) under dual antiplatelet therapy. The stent grafts were telescopically deployed in the cavernous ICA. The aneurysm was totally excluded from the circulation immediately after stent graft placement preserving the affected ICA. There were no peri- and postinterventional complications. Follow-up DSA at six months confirmed complete aneurysmal exclusion without any clinical sequelae. The stent grafts showed no in-stent stenosis or intima hyperplasia.

Giant intracranial ICA aneurysms are predominantly associated with clinically significant compression of adjacent cranial nerves. The results of endosaccular treatment (i.e. stent-assisted coil embolisation) of these aneurysms are promising with regard to the aneurysmal occlusion rates. Nevertheless, the mass effect of the aneurysms remains unchanged. The successful application of flow-diverters in this context has been proven in several publications. However, only a few published cases of stent grafts used to exclude intracranial aneurysms can be found in literature. In particular, the application of balloon-expandable stent grafts for treatment of intracranial aneurysms is currently discussed controversially.

The aim of this technical note is to present an alternative, technically feasible endovascular approach to exclude giant cavernous ICA aneurysms.

\section{2-P.09}

Category: Interventional

Presentation Type : Poster Presentation

Keywords : SAH, Vasospasm, PTA

Percutaneous Transluminal Angioplasty Using neck remodeling (compliant) balloon catheter for the treatment of severe vasospasm following intracranial aneurysm rupture.

$\underline{\text { Rokudai Sakamoto }}{ }_{-}{ }_{-}$, Masaru Hirohata ${ }^{2}$, Toshi $\mathrm{Abe}^{3}$, Motohiro Morioka ${ }^{1}$

Kurume Univeristy Neurosurgery Kurume-Japan ${ }^{1}$ Kurume University Neurosurgery Kurume-Japan ${ }^{2}$ Kurume University Radiology KurumeJapan $^{3}$

Introduction: Delayed cerebral vasospasm is a major cause of morbidity and mortality in aneurysmal subarachnoid hemorrhage in spite of many prophylactic medical treatment. Percutaneous Transluminal Angioplasty (PTA) of this condition offer a hope in preventing adverse outcome. The purpose of this study was to analysis the usefulness of PBA for the patients with severe cerebral vasospasm.

Material \& Method: Between 2003 to 2015, Fifty-six patients with severe vasospasm were treated with PTA. Within these patients 15 patients needed more than one PTA for vasospasm of other arteries. Total 143 arteries (ICA:57, M1 or M2:57, A1 or A2:22, VA BA or PCA: 7) were treated with PBA. PTA balloon catheters (Gateway: Striker, Unryu: Kaneka) were used in 7 arteries and other arteries were treated with neck remodeling or occlusion balloon catheters (Hyper-glide 4 x 10: EV3, Shouryu.4 x 10: Kaneka, Scepter C 4 x 10: Terumo, etc).

Results: PTA was accomplished in $97.2 \%$ of target arteries ( $82 \%$ of A1 or A2, $100 \%$ of other arteries). PTA balloon catheter or 0.014 inch guide wire compatible neck remodeling balloon catheters were necessary to treat $54 \%$ of ACA vasospsm. There was one clinical complication during PTA (intra operative rupture of ACA) but no re-spasm after BPA.

Conclusion: Percutaneous Balloon angioplasty is a safe and effective treatment for severe vasospasm after aneurysmal subarachnoid hemorrhage. 
2-P.10

Category: Interventional

Presentation Type : Poster Presentation

Multimodal experience in intra-arterial Acute Ischemic Stroke (AIS) treatment.

Edoardo Puglielli ${ }^{1}$, Ruggero Lattanzi ${ }^{1}$, Gianluigi Esposito ${ }^{1}$, Vincenzo Di Egidio

Asl Teramo Interventional Radiology Teramo-Italy ${ }^{1}$

Purpose: Endovascular reperfusion in AIS is safe and useful. Aim of the work is to report our experience in the endovascular multimodal thrombectomy.

Methods: 170 consecutive patients with AIS (mean age 52,3y, from April 2009 to May 2016), with classic on-set, undergoing to endovascular therapy using retrievable stents, thromboaspiration, intravenous and/or intra-arterial tissue plasminogen activator (t-PA) thrombolysis, PTA/ permanent stent placement. Basal CT, CTP/CTA and DSA were used for the imaging. Outcome and mortality were assessed after treatment. Patient's age, sex, aetiology, symptom, NIHSS score at presentation and $\mathrm{mRS}$, recanalization rates evaluated with TICI and complications were recorded and correlated using a multiple logistic regression analysis. Results: Median NIHSS at presentation was 22 (range 3-36). Sites of arterial occlusion before treatment were: M1 84/170 patients $(49.41 \%)$, intracranial carotid 12/170 patients (7.05\%), M2 22/170 (12.94\%), tandem occlusion 10/170 (5.88\%), extracranial internal carotid isolated occlusion 9/170 (5.29\%), P1 12/170 (7.05\%), basilar 21/170 (12.35\%). Therapeutic interventions were: a) simple stent-retriver embolectomy $145 / 170(85.29 \%)$; b) thromboaspiration $18 / 170(10.58 \%)$; C) pharmacologic cheap with (t-PA) $7 / 170$ (4.11\%). A TICI III was achieved in 128/ $145(88.27 \%)$ of the A group and in 14/18 (77.77\%) of the B group if compared to the $3 / 7(42.85 \%)$ of $\mathrm{C}(\mathrm{p}<0.005)$. Hemorragic suffusion occurred in one of the $\mathrm{C}$ group M1 occlusion patient that spontaneously resolved. At 6 months, good functional outcome (mRS 0-2) was observed in $63.29 \%(107 / 170)$; procedural mortality was $4.11 \%(7 / 170)$.

Conclusion: Multimodal endovascular approach in AIS has high recanalization rates, good functional outcome, with very low complications.

\section{2-P.11}

Category: Interventional

Presentation Type : Poster Presentation

Keywords : Vertebral artery, Dissecting aneurysm, stent assisted aneurysm embolization

Usefulness of stent assisted aneurysmal embolization for the treatment for unruptured vertebral artery dissecting aneurysm

Masaru Hirohata $^{1}{ }_{-}$, Rokudai Sakamoto ${ }^{2}$, Toshi Abe ${ }^{3}$, Hotohiro Morioka $^{1}$

Kurume University Neurosurgery Kurume-Japan ${ }^{1}$ Kurume Univesity Neurosurgery Kurume-Japan ${ }^{2}$ Kurume University Radiology KurumeJapan $^{3}$

Introduction: Vertebral artery dissecting aneurysm (VADA) contribute to both hemorrhagic and ischemic strokes. The Available endovascular surgical treatment modalities were destructive surgery (internal trap or parent artery occlusion) or reconstructive surgery (stent assisted aneurysm embolization). Internal trapping prevents rupture or growth of aneurysm, but has a risk of ischemic stroke. Reconstructive stent assisted aneurysm embolization have been reported as alternative.

The purpose of this study was to analysis the usefulness of Stent assisted aneurysm embolization for unruptured VADA.
Material and Method: Between 2002 to 2016, eighteen patients (M:F: 14:4; mean age 51.4 y.o.) with unruptured VADA were treated with Stent assisted aneurysm embolization. Indications of surgery for uuruptured VADA are aneurysmal growth within follow-up or larger than $10 \mathrm{~mm}$ aneurysm. Initial symptoms were occipital headache in eight, Asymptomatic in seven, brainstem infarction is two, lower cranial nerve palsy in one patient. Additional in stent balloon remodeling technique was used in 16 patients.

Results: The operative procedures were succeeded in all patients. The stent which used were Neuroform in nine, Enterprise in seven, LVIS Jr in two and coronary stent in two patients. Perioperative complication occurred in one patient (asymptomatic cerebellar infarction). Clinical outcome at discharge was mRS 0 in all patients. One year follow up DSA was achieved in 12 patients, one patients showed mild recanalization. Conclusion: Stent assisted aneurysmal embolization for unrupturd VADA is safe and feasible treatment. Although follow up periods was short, aneurysm recanalization was rare.

2-P.12

Category: Interventional

Presentation Type : Poster Presentation

Keywords :

3D vascular model is valuable in neurointervention.

$\underline{\text { Shigeru Nemoto }}^{1}$

Tokyo Medical and Dental University Endovascular Surgery Tokyo-Japan ${ }^{1}$

Purpose: 3D imaging is valuable in diagnostic neuroradiology and neurointervention. In coiling of the aneurysm, shaping of the microcatheter is a key to successful catheterization of the aneurysm. With $3 \mathrm{D}$ printer a $3 \mathrm{D}$ vascular aneurysm model is made according to the dicom data. Usefulness of this vascular model in coil embolization of cerebral aneurysm is examined.

Material and Method: An aneurysm vascular model is made of ABS resin with $3 \mathrm{D}$ printer according to dicom data of cerebral aneurysm. A microcatheter is shaped as same as this aneurysm vascular model. Microcatheter is navigated into the aneurysm and coils were inserted into the aneurysm. Results were compared with and without vascular model.

Results: Catheter shaping according to the vascular model leads to easy navigation into the aneurysm and to stable catheterization during coil insertion.

Conclusion: Catheter shaping according to the $3 \mathrm{D}$ vascular model is valuable in coil embolization of the aneurysm not only for beginners but also for experts. 3D vascular model is an extremely useful device in neurointervention of the cerebral aneurysms.

\section{2-P.13}

Category: Interventional

Presentation Type : Poster Presentation

Keywords : mechanical thrombectomy

Mechanical thrombectomy in acute ischaemic stroke: devicethrombus interaction in a pulsatile flow model of the MCA

Frank Lally ${ }^{1}$, Iris Grunwald ${ }^{2}$, Mahdi Saleh ${ }^{3}$, Scott Eaves ${ }^{1}$, Christine Roffe ${ }^{1}$

Keele University Institute for Science and Technology in Medicine Stoke-On-Trent-United Kingdom ${ }^{1}$ John Radcliffe Hospital NIHR Oxford Biomedical Research Centre Acute Vascular Imaging Centre 
Oxford-United Kingdom ${ }^{2}$ Charing Cross Hospital Clinical Decisions Unit London-United Kingdom ${ }^{3}$

Aims: The aim of this study is to compare performance characteristics of current stent-based thrombectomy devices in a controlled setting.

Materials and Methods: This is an in vitro study of the performance of five different thrombectomy devices (Aperio, Aperio 1.9F, Codman Revive SE, Solitaire FR ev3, and Stryker TREVO) in a model of the middle cerebral artery and its associated branches simulated to run under human conditions.

Results: There were a total of five individual experiments for each device with three attempts per experiment ( $\mathrm{n}=75$ potential attempts). The Solitaire took 2.8 mean attempts to restore flow while the Aperio $1.9 \mathrm{~F}$ and Trevo devices recanalized the occlusion in 1.8 attempts. Recanalization rates were $100 \%(5 / 5)$ for all devices except the Aperio where a rate of $80 \%(4 / 5)$ was achieved. The Aperio $1.9 \mathrm{~F}$ had the lowest embolization rate of $11 \%$ ( $1 / 9$ of all attempts) whereas the Trevo and Revive had the highest rates at 56\% (5/9 of all attempts). The Aperio was the fastest to recanalize the model artery (mean $33 \mathrm{sec}$; range 24-47 sec) whereas the Aperio 1.9F was the slowest (mean $241 \mathrm{sec}$; range 129477 sec; $\mathrm{p}<0.05)$.

Conclusion: Commercially available stentrievers are similar in terms of performance when used in a controlled setting. The Aperio 1.9F was significantly slower at recanalization than some of the other devices although displayed the lowest embolization rate. The speed of recanalization may need to be balanced against the risk of fragmenting the thrombus on retrieval, although this needs to be confirmed via in vivo trials.

\section{2-P.14}

Category: Interventional

Presentation Type : Poster Presentation

Keywords : Hyperplasia Flow-Diverter aneurysm

"Helical" Hyperplasia in Intracranial Flow-Diverter Stent: one case report

Carmine Sicignano ${ }^{1}$, Giuseppe Buono ${ }^{1}$, Luigi Delehaye ${ }^{1}$, Giuseppe La ${ }^{T}$ Tessa ${ }^{1}$, Vincenzo D' agostino ${ }^{1}$, Valeria Piscitelli ${ }^{1}$, Giovanni Sirabella ${ }^{1}$

P.O. Loreto Nuovo Neuroradiology Napoli-Italy ${ }^{1}$

We describe a single case of "helical" hyperplasia in a intracranial FlowDiverter (FD) stent (Pipeline Embolization Device, Covidien).

The patient was treated for an aneurysm of the Anterior Communicating Artery, by coiling associated to the "Twin Peds" procedure, that consists in positioning FDs in both Anterior Cerebral Arteries.

After one year the patient was asymptomatic and the DSA control was routinely performed; it showed a complex stenosys inside the FD stent, helical like, considered as hyperplasia; the parent vessel was normal as well the distal branches; the FD stent did not show any morphologic sign of deterioration or dislocation; the aneurysm was excluded; no systemic pathology was detected. For this reason we decided to continue the antiplatelet therapy; the corticosteroid therapy was introduced too, based on the cardiological considerations regarding the theory of an inflammatory role in the process of hyperplasia inside the coronary stents. We plan father MRI and DSA investigations to evaluate.

This report is motivated by the complex morphology of the stenosys, never seen before in our experience and not reported in the current litterature; for this reason we are not able to predict if it's a case of reversible hyperplasia, usually seen after stenting, or something different.
2-P.15

Category: Interventional

Presentation Type : Poster Presentation

Keywords : glomus tympanicum, embolisation, CT

\section{Glomus tympanicum - diagnosis and treatment Case report} $\frac{\text { Aleksandar Jovanovski }^{1}}{\text { Tijana Kosanović }^{1}}-$ Dejan Kostić $^{1}$, Igor Sekulić ${ }^{1}$, Mišel Radulac ${ }^{1}$,

Military Medical Academy Institute of radiology Belgrade-Serbia ${ }^{1}$

Glomus tympanicum is the most common primary neoplasm of the middle ear and the second most common tumour of the temporal bone.

The most common presenting symptoms are conductive hearing loss and pulsatile tinnitus.

CT and MRI scans are the primary imaging modalities.

Superselective angiography with embolisation aids in identifying and blocking the feeding arteries.

Surgery, embolisation and radiotherapy are the modalities oftreatment.

A 50 year old female presented with a history of pulsatile tinnitus and progressive, diminished, right-sided hearing of 6 months duration.

Clinical examination revealed a pulsatile mass in the middle ear. She was subjected to further evaluation by CT imaging of the temporal bones which demonstrated an enhancing mass in the middle ear.

Superselective angiographic study showed a vascular tumour in the right middle ear supplied by a branch of of a.meningea media and mainly by branch of occipital artery, which was selectively catheterised and embolised using Embozen particles.

A surgical exploration was done one day after embolisation.

It was excised in toto, Blood loss was minimal.

Surgery, embolisation and radiotherapy are available modalities of treatment. Pre-operative tumor embolization is useful, by reducing blood supply to the tumour, diminishing the need for transfusion.

2-P.16

Category: Interventional

Presentation Type : Poster Presentation

Keywords :

Endovascular treatment of intra cranialvertebral dissecting aneurysms: pattern of disease and therapeutic options in a single centre.

Chinthaka Appuhamy ${ }^{1}$, Wickly Lee ${ }^{1}$, Francis Hui ${ }^{1}$

National Neuroscience Institute Neuroradiology Novena-Singapore ${ }^{1}$

Aim: Intracranial vertebral dissecting aneurysm (VDA) is a rare but potentially devastating condition with a generally poor prognosis. The aim of this study is to analyse the pattern of disease and therapeutic options employed in the endovascular treatment.

Method: The study involved patients who underwent endovascular treatment of VDA between January 2011 and May 2016. The World Federation of Neurosurgeons (WFNS) grading and the modified Fisher scale were used to evaluate the clinical status and extent of the subarachnoid haemorrhage (SAH). Relationship to the posterior inferior cerebellar artery (PICA) was analysed. 
Modified Rankin Scale was used to assess the post treatment outcome.

Results: Out of 30 patients, there were 15 ruptured and 15 unruptured aneurysms. Of the ruptured aneurysms, $10(71 \%)$ were distal to the PICA, 2 (14\%) involved the PICA and 2 (14\%) were proximal to the PICA. Among the unruptured aneurysms, $4(27 \%)$ were distal to the PICA, 4 (27\%) involved the PICA and 7 (47\%) were proximal to the PICA.

Among patients with SAH, 15 (94\%) underwent internal trapping and $1(6 \%)$ was stented. The WFNS score among SAH patients was poor (4 and 5) in $10(67 \%) .7(47 \%)$ had favourable outcomes (mRS of 2 or less).

In the unruptured group, clinical outcome was favorable in all $15(100 \%)$ patients although $2(13 \%)$ showed small medullary infarcts.

Conclusion: Endovascular treatment by arterial trapping or stenting carried a favorable immediate result with higher success rates and low incidence of complications. However the endovascular treatment technique should be individualized according to the disease pattern.

\section{2-P.17}

Category: Interventional

Presentation Type : Poster Presentation

\section{Keywords :}

Endovascular Treatment of Head and Neck Tumours Complicated with Vascular Invasion.

\section{Chinthaka Appuhamy ${ }_{-}^{1}$, Ashank Bansal ${ }^{1}$, Wickly Lee ${ }^{1}$}

National Neuroscience Institute Neuroradiology Novena-Singapore ${ }^{1}$

Aim: Vascular invasion by head and neck tumours is a lethal complication of malignancy. Surgical treatment options in this patient population are limited and potentially hazardous making endovascular management the preferable mode of treatment. The aim of this study is to evaluate the clinical and technical factors related to the outcome of endovascular treatment.

Methods: Patients with head and neck malignancies who had undergone endovascular treatment between January 2013 and April 2016, were evaluated.

Results: 13 patients with head and neck malignancies underwent endovascular evaluation from which 7 were managed endovascularly. Three patients had nasopharyngeal carcinoma (NPC), two had squamous cell carcinoma (SCC) and one had laryngeal carcinoma (LC). The common carotid artery (CCA) was involved in two patients whereas internal (ICA) and external carotid (ECA) arteries were involved in four patients each. Two patients demonstrated active bleeding at the time of embolization. Interventions were carried out using covered stents, detachable coils and liquid embolic material. Technical success of the procedure was $100 \%$. There were no immediate procedural complications however one patient developed left hemispheric syndrome due to watershed infarctions following closure of the ipsilateral internal carotid artery using a vascular plug. At an average follow-up of 3 months (range $<1-14$ months), four patients were alive, two had died as a result of underlying disease, and one had died of other causes.

Conclusion: Head and neck tumours with vascular invasion is associated with significant morbidity, however endovascular management achieves immediate hemostasis with low rate of periprocedural complications.

\section{3- Head \& Neck}

3-P.01

Category: Head \& Neck

Presentation Type : Poster Presentation

Keywords : retinoblastoma, 3.0 Tesslas magnetic resonance,optic nerve invasion, choroidal spread

The contribution of high field MRI in the diagnosis of extraocular extension in retinoblastoma: a comparative study

Aranzazu Royo ${ }^{1}$, Alberto Alvarez-Muelas ${ }^{1}$, Cristina Utrilla ${ }^{1}$, Jose Abelairas $^{2}$, Nelson Mauricio Buitrago ${ }^{1}$, Amelia Fernandez-Zubillaga ${ }^{1}$, Juan Alvarez-Linera ${ }^{3}$, Gonzalo Garzón ${ }^{4}$

Hospital Universitario La Paz Neuroradiology Madrid-Spain ${ }^{1}$ Hospital Universitario La Paz Pediatric Ophtalmology Madrid-Spain ${ }^{2}$ Hospital Ruber Internacional Neuroradiology Madrid-Spain ${ }^{3}$ Hospital Universitario La Paz Radiology Department Madrid-Spain ${ }^{4}$

Objectives: Review MRI criteria for extraocular extension and progression. Evaluate the usefulness of MRI, 1.5 ando 3.0T MRI, in the final decision of eye enucleation when dissemination or progression is uncertain Introduction: Knowledge of extraocular extension of retinoblastoma choroid, sclera, optic nerve and orbit is vital in staging the tumor to decide the appropriate treatment.

According to the literature, the accuracy of magnetic resonance images (MRI) to assess extraocular extension in this disease remains controversial

Material and Methods: 13 patients with retinoblastoma (24 eyes: 11 bilateral, 2 in patients with a single eye) under chemotherapy were evaluated with 1.5 and 3T MRI within 1 month.

Orbital extension, both intraocular (choroidal or scleral spread, vitreous seed, and involvement of the anterior segment) and extraocular (postlaminar optic nerve involvement and extrascleral invasion) was evaluated.

11 eyes were enucleated and 2 patients were followed-up. Correspondence between MRI-based indication for enucleation and histology was also studied.

Results: 3TMR showed better sensitivity for detection of extraocular extent but it also showed more false positive results in optic nerve and choroid involvement.

Conclusion: 3TMR allows more reliable information before enucleation in patients with retinoblastoma concerning choroid and optic nerve invasion, anterior chamber infiltration and specially evaluating the morphology of the tumor, but it also shows more false positive results when evaluating optical nerve and choroid-scleral extent. Despite the small number of the series we infer that indirect criteria such as choroid-retinial \& choroid-scleral interruption should be reviewed due to the higher sensitivity of this technique.

\section{3-P.02}

Category: Head \& Neck

Presentation Type : Poster Presentation

Keywords : MRI, contrast agent, safety, adverse events, NSF

SECURE study: observational post-marketing study on the safety profile of gadoteric acid - Final results in 35,499 patients

$\underline{\text { De-Hua Chang }}{ }^{\text {I }}$

University Clinic Cologne Radiology Cologne-Germany ${ }^{1}$ 
Purpose: To prospectively assess the safety profile of gadoteric acid (DOTAREM ${ }^{\circledR}$, Guerbet, France) and the incidence of Nephrogenic Systemic Fibrosis (NSF) in routine practice.

Materials and Methods: A worldwide observational post-marketing study was undertaken to collect safety data in adults and children with or without renal impairment, who were scheduled to undergo routine contrast-enhanced Magnetic Resonance Imaging (MRI) with gadoteric acid. Demography, risk factors, indication(s) for MRI and occurrence of adverse events (AE) were recorded. A 3-month follow-up period was planned for patients with at least moderate renal impairment (eGFR $<60 \mathrm{~mL} / \mathrm{min} / 1.73 \mathrm{~m}^{2}$ ) in order to detect any suspicion of NSF.

Results: A total of 35,499 patients were analyzed (female: $53.1 \%$; mean age: 49.5 years; range: $0-98$ years). Central nervous system was the primary indication (56.8\%) for contrast-enhanced MRI. The main risk factors were any stage of renal impairment (14.7\%) and hypertension $(11.9 \%)$. A total of 70 AEs were observed in 44 patients $(0.12 \%)$, mainly urticaria $(0.03 \%$ of patients), nausea $(0.02 \%)$ and vomiting $(0.01 \%)$. Among them, 38 AEs reported in 32 patients $(0.09 \%)$ were considered as adverse drug reactions. Nine adult patients $(0.03 \%)$ experienced serious AEs. Moderate to severe renal impairment was reported in 514 patients (1.4\%), 92.6\% of them were followed-up with no suspicion of NSF observed.

Conclusion: This final analysis in 35,499 patients of all ages confirms the excellent safety profile of gadoteric acid in routine practice.

\section{3-P.03}

Category: Head \& Neck

Presentation Type : Poster Presentation

Keywords : Inner ear, Hemorrhage, Sensorineural hearing loss

Labyrinthine hemorrhage detected on magnetic resonance image as a cause of sudden profound sensorineural hearing loss

\section{Dong Woo Park $^{1}$}

Hanyang University Guri Hospital Radiology Guri-Korea, South ${ }^{1}$

Purpose: The purpose of this study is to evaluate the value of magnetic resonance image (MRI) in acute labyrinthine hemorrhage as the cause of sudden profound sensorineural hearing loss, and to correlate the high signals in the labyrinth with clinical features

Materials and Methods: MRIs performed in 99 patients with sudden sensorineural hearing loss were retrospectively analyzed. The high signals in the labyrinth on MRI were evaluated to correlate with the clinical and audiometry features of patients. MR imaging includes high resolution T1-weighted, 3D FLAIR volume isometric turbo spin echo acquisition (VISTA) and enhanced T1-weighted images.

Results: High signals in the labyrinth on MRI with high resolution T1-weighted, 3D FLAIR VISTA with no enhancement were demonstrated in $5(5.1 \%)$ patients among 99 patients with sudden sensorineural hearing loss, which were consistent with labyrinthine hemorrhage.

Clinical features of these 5 patients were characterized by sudden profound sensorineural hearing loss and vestibular dysfunction, resulting in permanent hearing impairment.

Conclusion: MRI with high resolution T1-weighted, 3D FLAIR VISTA and enhanced T1-weighted images could demonstrate labyrinthine hemorrhage as the cause of sudden profound sensorineural hearing loss. Labyrinthine hemorrhage presenting as high signals in the labyrinth could be correlated with clinical features with sudden, profound sensorineural hearing loss and vestibular dysfunction, resulting in permanent hearing impairment.
4- Spine

4-P.01

Category: Spine

Presentation Type : Poster Presentation

Keywords : spinal cord, distinguishing of primary demyelination

Importance of analysis of spinal cord in primary demyelinating disease suggestive cases

$\underline{\text { Svjetlana Jefic }}_{-}{ }_{-}$, Daliborka Maric ${ }^{1}$

University Clinical Centre of Repablic of Srpska Department of Clinical Radiology Banja Luka-Bosnia and Herzegovina ${ }^{1}$

Abstract: Whole neuroaxis should be considered as the site of pathomorphological changes related to primary demyelinating diseases (PDDs).

Some of PDDs involve only the brain (TD - tumefactive demyelination), some of them involve only spinal cord (TM - transverse myelitis, CIDP chronic inflammatory demyelinating polyneuropathy, GBS -GuillianBarre Syndrome), but a lot of PDDs involve spinal cord as well as brain (MS - Multiple Sclerosis, NMO - Neuromyelitis Optica, ADEM - Acute Disseminated Encephalomyelitis, CIS - clinical isolated syndrome).

These conditions can mimic each other having similar pathomorphological features and clinical course. Also, there may be overlapping of clinical and neuroimaging findings between PDDs and other pathologies.

Identification of specific PDDs is essential because it implies accurate therapy that can modify clinical course, prevent or delay level of disorder. There are a few patterns of PDDs morphological changes in the spinal cord which finding - in combination with finding of particulare changes in the brain - can increase certainty of diagnosis and in this specific context for individual patient can play a role of biomarker.

Understanding of these patterns and necessity of exploration of spinal cord in some cases can lead to distinguishing of PDDs.

Neuroimaging analysis should respect complementary of findings both in brain and spinal cord.

Presentation shows our cases with PDDs where spinal cord findings led to establishing of diagnosis.

\section{4-P.02}

Category: Spine

Presentation Type : Poster Presentation

\section{Keywords :}

\section{Diastematomyelia in adults: report of two cases.}

$\underline{\text { Laura Saitta }}{ }^{1}$, Caterina Lapucci ${ }^{2}$, Lara Castelletti ${ }^{1}$, Lucio Castellan ${ }^{1}$

San Martino IST Hospital Neuroradiology Genoa-Italy ${ }^{1}$ San Martino IST Hospital Neurology Genoa-Italy ${ }^{2}$

Diastematomyelia is a rare congenital spinal malformation, characterized by a localized longitudinal partial or complete division of the spinal cord. We present two cases of diastematomyelia in two women in whom the diagnosis was made in the first place at the age of 71 and 63 years respectively.

Case 1: a 71 years old woman, suffering from hyposthenia of her left lower limb since childhood and previously diagnosed as a consequence of poliomyelitis, presented to our observation for worsening of her gait disturbance. The MRI showed fusion of the L1-L2-L3 vertebral bodies, with defective fusion of the posterior vertebral elements of L4, a low lying conus and separation of the spinal cord into two hemicords in the T10-L5 tract with an intervening osseous septum in the L1-L3 tract. 
Case 2: a 63 years old woman, who complained of progressive gait disturbance related to mild weakness of the right lower limb in the past ten years, referred to our hospital with severe pain in her lower limbs. The MRI showed a low lying partially split cord at the L1-L2 level with a single dural sac containing both hemicords. Disraphic defects of the posterior vertebral elements were also present in the L1-L4 tract.

Dyastematomyelia is usually diagnosed early in life, while the diagnosis during adulthood is infrequent. In adults patients the symptoms appear more frequently when the intervening degenerative disease of the spine awakens the underlying malformation.

\section{5 - Pediatric}

\section{5-P.01}

Category: Pediatric

Presentation Type : Poster Presentation

Keywords : acute encephalopathy, AESD, pediatric, MR spectroscopy

Disrupted glutamate-glutamine cycle in acute encephalopathy with biphasic seizures and late reduced diffusion (AESD)

$\underline{\text { Jun-ichi Takanashi }}{ }^{1}$, Eri Oguni ${ }^{1}$, Masashi Mizuguchi ${ }^{2}$

Tokyo Women's Medical Univ. Yachiyo Medical Ctr. Pediatrics YachiyoJapan ${ }^{1}$ Graduate School of Medicine, the University of Tokyo Developmental Medical Sciences Tokyo-Japan ${ }^{2}$

Objective: Acute encephalopathy with biphasic seizures and late reduced diffusion (AESD) is the most common subtype of infectious pediatric encephalopathy in Japan. It is sometimes difficult to make an early diagnosis of AESD; excitotoxicity is postulated to be the pathogenesis based on elevated glutamine (Gln) and glutamate (Glu) complex $(\mathrm{Glx}=\mathrm{Glu}+$ Gln) observed on MR spectroscopy. It is uncertain whether Gln or Glu contributes to the elevated Glx, or whether MR spectroscopy is useful for an early diagnosis.

Methods: Seven Japanese patients with AESD (five boys and two girls, 11 month to 1 year of age) were enrolled in this study. MR spectroscopy was acquired from the frontal white matter(TR of $5000 \mathrm{msec}$, TE of 30 $\mathrm{msec}$ )with a 1.5 or 3.0 tesla scanner. MR spectroscopy was performed 4 times for two patients, 3 times for 1 patient, twice for 3 patients, and once for 1 patient. Quantification of Glu and Gln was performed using LCModel.

Results: Glu was elevated in 4 of 5 studies on days 1-4 before the bright tree appearance on DWI, and became normal or low afterwards. Gln was normal in 3 studies on days 1-2, elevated in all 9 studies on days 4-12, and became normal or low afterwards.

Conclusion: These findings suggest that elevated Glx observed on MR spectroscopy may be useful for an early diagnosis. Acute Glu elevation changes to subacute Gln elevation, suggesting a disrupted Glu-Glncycle may play an important role.

\section{5-P.02}

Category: Pediatric

Presentation Type : Poster Presentation

Keywords : Epilepsy, MRI

Magnetic Resonance abnormalilties in epileptic children and adolescence: 5 year experience

$\underline{\text { Hye Jeong Kim }}{ }^{1}$, Hee Yeong Kim ${ }^{1}$, Ji Young Woo ${ }^{1}$, Ik Yang ${ }^{1}$
Kangnam Sacred Heart Hospital radiology Seoul-Korea, South ${ }^{1}$

Magnetic resonance imaging (MRI) is an important tool to evaluate the structural abnormalities in patients with epilepsy. In this study we surveyed MRI abnormalities in children and adolescence with epilepsy.

From 2010 to 2015,420 patients age 1 to 20 years presenting with epilepsy were included in this retrospective study. There were $194(46.2 \%)$ women and $226(53.8 \%)$ male.

Of these, MRI abnormalities was seen in $77(18.3 \%)$ patients and consisted of benign cyst $(22.1 \%)$, encephalomalacia (15.6\%), congenital malformation (13.0\%), vascular abnormalities (7.8\%), pituitary lesion $(7.8 \%)$, myelination abnormalities $(7.8 \%)$, brain tumor (5.2\%), infection (3.9\%), brain atrophy (3.9\%), hippocampal sclerosis $(2.6 \%)$ and others $(10.4 \%)$.

All MRI abnormalities are not associated with epilepsy. However MRI scan is useful to detecting structural abnormalities and it help to determine the treatment.

\section{5-P.03}

Category: Pediatric

Presentation Type : Poster Presentation

Keywords : pediatric, children, diffusion tensor imaging, tractography, corpus callosum

Diffusion tensor imaging and fiber tractography of the congenitally thickened corpus callosum

Katarzyna Nowak ${ }^{1}$, Elzbieta Jurkiewicz ${ }^{2}$, Sylwia Chelstowska ${ }^{3}$, Katarzyna Kotulska-Jozwiak ${ }^{3}$, Piotr Pawliszak ${ }^{1}$, Krzysztof Sadowski ${ }^{3}$, Iwona Pakula-Kosciesza ${ }^{4}$

The Children's Memorial Health Institute Department of Diagnostic Imaging Warsaw-Poland ${ }^{1}$ The Children's Memorial Health Institute Departmen of Diagnostic Imaging Warsaw-Poland ${ }^{2}$ The Children's Memorial Health Institute Department of Neurology Warsaw-Poland ${ }^{3}$ The Children's Memorial Health Institute Depertment of Diagnostic Imaging Warsaw-Poland ${ }^{4}$

Introduction: Diffusion tensor imaging (DTI) is the best method for visualization precise brain anatomy and for evaluation congenital anomalies. DTI gives additional information about microstructures of white matter tracts and their function. The normal corpus callosum has four named regions, genu, body and splenium from anterior to posterior and the small rostrum located under the genu. 10 cases of abnormal thick corpus callosum (CC) were retrospectively evaluated by MR imaging, diffusion tensor imaging and fiber tractography.

Material and Methods: All MR examinations were obtained on the $1.5 \mathrm{~T}$ superconducting system using 8 -channel phased-array head coil. DTI was obtained using 12 directions, with $b=0,800$ $\mathrm{s} / \mathrm{mm} 2$.

We analyzed MR and DTI examinations of 10 patients ( 5 boys and 5 girls), aged from 1.5 to 13 years (mean age 10.5).

2 patients were diagnosed with NF1, one with hemi- megalencephaly, and the others had no pathological changes in the brain MRI

Any of our patients did not meet the clinical diagnostic criteria of Cohen syndrome, M-CMTC (macrocephaly-capillary malformation) and megalencephaly-polymicrogyria-mega-corpus callosum (MEG-PMGMegaCC) syndrome.

Results: MR images of all our patients showed focal or complete callosal thickening and shortened longitudinal dimension. 
DTI and tractography of three girls (aged 10,11, and 12 years) presented additional fibers above corpus callosum most likely represent heterotopic cingulum

Conclusions: In our opinion, diffusion tensor imaging and tractography should be performed in children with abnormalities in the shape of the corpus callosum.In the normal conditions, no running fibers lying above or below corpus callosum.

\section{5-P.04}

Category: Pediatric

Presentation Type : Poster Presentation

Keywords : pediatric, glioblastoma, perfusion weighted imaging, diffusion weighted imaging

Pediatric glioblastoma - utility of the perfusion and diffusion weighted imaging

Sylwia Chelstowska ${ }^{1}$, Elzbieta Jurkiewicz ${ }^{2}$, Katarzyna Nowak ${ }^{1}$, Bozenna Dembowska-Baginska ${ }^{3}$, Marta Perek-Polnik ${ }^{4}$, Katarzyna Malczyk ${ }^{1}$, Piotr Pawliszak ${ }^{5}$

The Children Department of Diagnostic Imaging Warsaw-Poland ${ }^{1}$ The Children Departmen of Diagnostic Imaging Warsaw-Poland ${ }^{2}$ The Children Deprtment of Oncology Warsaw-Poland ${ }^{3}$ The Children Department of Oncology Warsaw-Poland ${ }^{4}$ The Children Depertment of Diagnostic Imaging Warsaw-Poland ${ }^{5}$

Aim: We evaluate and compare perfusion and diffusion parameters from dynamic contrast-enhanced (DCE), dynamic susceptibility contrast (DSC) and apparent diffusion coefficient (ADC) characteristic for pediatric glioblastoma. Material and Methods: MR examinations of 20 patients (11 male and 9 female) aged 1.9y.-17.6y with histologically proven glioblastoma were retrospectively analyzed.

Statistical analysis was performed using Origin software. The Spearman test was used to calculate rank correlation coefficients between corresponding values of Ktrans, rCBV, and ADC.

All MRI studies were performed using a 1,5T unit.

Results: Ktrans, rCBV, and ADC parameters were calculated from tumor mass and normal appearing brain tissue. The results for $\mathrm{rCBV}(344,9$ versus $162,55, p$ value $=0,0157)$ and $A D C(118,333$ versus $81,35, p$ value $=0,00129$ ) parameters were statistically significant.

The Ktrans parameter was not relevant as a diagnostic factor for glioblastoma patients $(0,045$ versus 0,016 , $\mathrm{p}$ value $=0,1436)$.

The Spearman rank correlation coefficients were respectively: Ktrans versus $\mathrm{rCBV}(-0,1, \mathrm{p}$ value $(0,872)$, Ktrans versus $\mathrm{ADC}(0.8, \mathrm{p}$ value $(0,104), \mathrm{rCBV}$ versus ADC $(-0,006$, p value 0,986$)$.

Conclusion: The Ktrans parameter did not show significantly different values for Mann-Whitney $U$ test but it presented strong positive correlation between Ktrans and ADC results. Both DSC and DCE perfusion should be considered in evaluation process of glioblastoma in children.

\section{5-P.05 \\ Category: Pediatric \\ Presentation Type : Poster Presentation}

Keywords : Neonatal cerebral sinovenous thrombosis, magnetic resonance venography (MRV)

Imaging findings in neonatal cerebral sinovenous thrombosis: Review of 4 years experience of a national tertiary referral hospital in Spain.

Nelson Mauricio Buitrago Sánchez ${ }^{1}$, Amelia Fernández Zubillaga ${ }^{1}$, Malaika Cordeiro Alcaine ${ }^{2}$, Cristina Utrilla Contreras ${ }^{1}$, Arancha Royo Orejas $^{1}$, Gonzalo Garzón Moll ${ }^{3}$
Hospital Universitario La Paz Neuroradiology Madrid-Spain ${ }^{1}$ Hospital Universitario La Paz Neonatal Intensive Care Unit Madrid-Spain ${ }^{2}$ Hospital Universitario La Paz Vascular and Interventional Radiology Madrid-Spain ${ }^{3}$

Neonatal cerebral sinovenous thrombosis (CSVT) is a rare and potentially lethal disorder, that can lead to stroke, epilepsy, cognitive and motor impairment in survivors. Cerebral magnetic resonance (MR) and MR venography (MRV) are the imaging tests of first choice in order to confirm or rule out CSVT.

We collected the data from our neonatal intensive care unit during the last 4 years, reviewing the brain MR images of 15 cases of neonatal venous thrombosis diagnosed in our institution. MRV is based on detecting flow signal from sinovenous system. The diagnosis of thrombosis is based on the absence of this signal. Common pitfalls in interpreting this flow signal, like a T1-shine-through phenomenon in subacute thrombi (due to the fact that contains methemoglobin), may yield false negative results in MRV, with the catastrophic consequences of an untreated CSVT. Based on the foregoing, conventional MR and MRV should always be interpreted together with the purpose of avoiding potential misinterpretations.

This review aims to describe the imaging findings in neonatal CSVT, the affected venous structures, the related brain parenchymal lesions in each case and to emphasize the importance of detecting subtle signs of CSVT in newborns in both conventional MR examination and MRV.

\section{5-P.06}

Category: Pediatric

Presentation Type : Poster Presentation

\section{Keywords :}

Microcephaly caused by Zika virus - MRI of the fetal brain and correlation with neuropathology

Tina Vipotnik Vesnaver ${ }^{1}$, Nataša Tul ${ }^{2}$, Jernej Mlakar ${ }^{3}$, Sara Mehrabi ${ }^{4}$, Tatjana Avšič Zupanc ${ }^{3}$, Mara Popović ${ }^{3}$

University Clinical Centre Institute of Radiology Ljubljana-Slovenia ${ }^{1}$ University Clinical Centre Dept of Perinatology Ljubljana-Slovenia ${ }^{2}$ Faculty of Medicine Institute of Pathology Ljubljana-Slovenia ${ }^{3}$ Policlinico G.B. Rossi Institute of Radiology Verona-Italy ${ }^{4}$ Faculty of Medicine Institute of Microbiology and Immunology Ljubljana-Slovenia ${ }^{5}$

Background: In December 2015, a possible association between Zika virus (ZIKV) infection during pregnancy and fetal brain malformations was suspected. Several papers published thereafter strengthened this association. In this case report we compare fetal MRI with post mortem neuropathology in a confirmed case of microcephaly associated with ZIKV.

Case presentation: A 25-year-old woman was reffered to our hospital due to fetal brain anomalies noticed in the second trimester of pregnancy. In 13 week of pregnancy she showed symptoms of ZIKV infection.

The main neuroimaging findings were micrencephaly, ventriculomegaly, severely delayed gyration with areas of suspected polymicrogyria, cystic encephalomalacia and calcifications at the cortico-subcortical border. Termination of pregnancy and autopsy at 33 weeks of pregnancy was performed. Neuropathological findings were in accordance with the imaging with the exception of polymicrogyria that wasn't present. Calcifications at the corticosubcortical border, neuronal heterotopia and thickened subarachnoid space filled with gliotic tissue that was bursting through the damaged pial surface gave the microscopical appearance of focal cobblestone lissencephaly. Discussion: Our case shares several imaging features with other reported ZIKV cases with predilection for frontal and parietal lobes, cystic encefalomalacia and multifocal subcortical calcifications, but to the best of our knowledge no case of prenatal infection related microscopic appearance of cobblestone type lissencephaly has been reported yet. 
The mechanism of ZIKV induced development of microscopical appearance of focal cobblestone lissencephaly remains to be elucidated.

\section{5-P.07 \\ Category: Pediatric \\ Presentation Type : Poster Presentation}

Keywords : Severe cerebellar hypoplasia, open spinal dysraphism, Chiari malformation

Particularly severe hypoplastic cerebellum located in upper cervical spinal canal associated with open spinal dysraphism - is it Chiari II malformation ? - A case report

$\underline{\text { Marijana Karlović-Vidaković }}{ }^{1}$, Zlatko Kolić ${ }^{2}$

Clinical Hospital Mostar Neuroradiology Departement Mostar-Bosnia and Herzegovina ${ }^{1}$ Special Clinic Medico Neurosurgery Departement Rijeka-Croatia $^{2}$

Chiari II malformation is a complex developmental malformation of the central nervous system characterized by a small posterior fossa and downward displacement of the cerebellum and brainstem through an enlarged foramen magnum. It is almost uniquely associated with open spinal dysraphism.

A female newborn was admitted at the Pediatric Department of our Hospital with a large lumbosacral myelomeningocele located at the level of L2-S1.Ten hours after birth the myelomeningocele surgery was done. Initial neuroaxis MRI was done at the age of 41 days, and the follow up MRI at the age of one year.

MRI examination was consistent with severe hypoplastic cerebellum, elongated and shaped like a spindle, located in the upper cervical spinal canal. The posterior fossa was extremely small and empty, with abnormally low position of the tentorium and consequently low insertion of the confluence of sinus, just above the enlarged foramen magnum. Downward displacement of a slit fourth ventricle was found.

The midbrain was hypoplastic and elongated, as well as the tectal plate, and an atipical position of cranial nerves was found.

Supratentorial, a broad spectrum of changes were noticed - prominent massa intermedia, hypoplastic corpus calosum associated with hydrocephalus and cortical disorganization, as well as giral interdigitation, etc. The question arises whether this condition is consistent with Chiari malformation type II or could it in fact be the Chiari IV type?

\section{5-P.08 \\ Category: Pediatric \\ Presentation Type : Poster Presentation}

Keywords : Rasmussen, encephalitis, MRI

\section{Rasmussen Encephalitis}

Hakan Cebeci ${ }^{1}$, Ibrahim Guler ${ }^{1}$, Huseyin Ozbiner ${ }^{1}$, Yahya Paksoy ${ }_{-}$

Selcuk University Faculty of Medicine Radiology Konya-Turkey ${ }^{1}$

Introduction: Rasmussen encephalitis is a rare disease most often characterised by single hemispheric, progressive inflammatory disease causing refractory focal epilepsy, cognitive decline and progressive neurological deficit. The aetiology remains unknown. Mostly presenting symptom is the focal motor seizures. It is mainly a childhood disease with the avarege onset age of 6-8 years. Rasmussen encephalitis can be diagnosed based on clinical, EEG and MRI findings (5,6, (dir). We aimed to present a case of Rasmussen encepahlitis diagnosed with clinical criteria and characteristic magnetic resonance findings.
Case: A 15-year-old girl with no medical history presented with a focal motor convulsions affecting right upper extremity. MRI of brain revealed left sided hemiatrophy and white matter changes affecting frontal, parietal, temporal and occipital lobes. Rasmussen encephalitis was diagnosed based on clinial and radiological findings.

Discussion: Rasmussen encephalitis is a medically refractory epileptic condition of childhood age that typically begins with focal seizures that invariably progress to a state of hemiparesis, medically intractable epilepsy, and cognitive decline. This disease is generally affects children. The diagnose of rasmussen encephalitis can be made according to clinical and radiological findigs with no need to biopsy. Awareness of MRI findings of this disease is helpful for early diagnose.

\section{5-P.09}

Category: Pediatric

Presentation Type : Oral Presentation

Keywords : pediatric, multiple sclerosis, children,

Pediatric multiple sclerosis - magnetic resonance in children under the age of 11 .

Elżbieta Jurkiewicz $^{1}$, Katarzyna Nowak ${ }^{2}$, Katarzyna Kotulska-Jozwiak ${ }^{3}$, Magdalena Maciejewska-Kotz ${ }^{1}$, Joanna Debska ${ }^{1}$, Julita Borkowska ${ }^{3}$

The Children's Memorial Health Institute Department of Diagnostic Imaging Warsaw-Poland ${ }^{1}$ The Children's Memorial Health Institute Departmen of Diagnostic Imaging Warsaw-Poland ${ }^{2}$ The Children's Memorial Health Institute Department of Neurology Warsaw-Poland ${ }^{3}$

Introduction: Multiple sclerosis (MS) is diagnosed in 2-10\% of cases in children under 16 years of age, and in $1-5 \%$ before 11 years of age. Demyelinating lesions visible on MRI of the brains' children with MS may differ from the lesions described in adult patients. The aim of this presentation is to show the diversity of MR images of the brain and the spinal cord in younger children.

Material and Methods: We present ten children (6 girls and 4 boys), aged 7 to 9 years and 6 months. Clinical evaluation of each patient included a neurological examination, EDSS scale, examination of cerebrospinal fluid, evoked potentials study.

MRI analyzes the number, location, morphology and the presence of contrast enhancement of lesions located in the brain and spinal cord.

Results: Infra- and supratentorial lesions were observed in all patients, lesions in the spinal cord of $40 \%$ children. Besides the typical demyelination changes, leucodystrophy-like, giant, tumefactive forms and the presence of pseudocysts were detected. Most of the lesions in the spinal cord were located in the lower part of the thoracic spine.

Conclusions: The brain MR examinations of the youngest children demonstrate a considerable diversity. The number of visible changes in the MRI studies correlates with the presence of symptoms and the degree of neurological deficits in EDSS. Due to the lack of the typical picture of MS in children the differentiation diagnosis should consider a wide range of diseases.

\section{5-P.10}

Category: Pediatric

Presentation Type : Poster Presentation

Keywords : Extrapontine myelinolysis, pediatric brain, MRI

Unusual case of pontine and extrapontine myelinolysis in a child with adrenal insufficiency

Nikola Boban $^{1}$, Dusko Kozic ${ }^{2}$, Marija Knezevic-Pogancev ${ }^{3}$, Jasmina Boban $^{2}$, Natasa Kovac ${ }^{3}$ 
Clinical Center of Vojvodina Radiology Department Novi Sad-Serbia ${ }^{1}$ Oncology Institute of Vojvodina Diagnostic Imaging Center Sremska Kamenica-Serbia ${ }^{2}$ Institute for Children and Adolescent Healthcare of Vojvodina Neurology Department Novi Sad-Serbia ${ }^{3}$

Introduction: We present a case of pontine and extrapontine myelinolysis with a favorable outcome after osmotyc demyelination syndrome confirmed on MRI.

Case Report: An 11-year-old girl with well controled adrenal insufficiency presented with acute prostration and incomprehensive verbal response, after a short period of physical stress and self-reducted doses of substitutional therapy. After the admission to ICU, contrast MRI was performed showing extensive symmetrical areas of $\mathrm{T} 2$ hyperintnesity in corpus callosum and deep frontoparietal white matter, resembling the corticospinal tract. Only two small discrete foci were visible in pons. All the lesions showed marked restricted diffusion in the means of cytotoxic oedema. After the readministration of substitutional therapy, clinical state of the patient improved rapidly. On control MRI, total regression of all described lesions was observed except for the lesion in splenium of corpus callosum.

Discussion: Osmotyc demyelination syndrome usually involves pons and extrapontine sites such as basal ganglia, thalami and deep white matter, while the involvement of corpus callosum and corticospinal tract, as described here, is rare.

\section{5-P.11 \\ Category: Pediatric \\ Presentation Type : Poster Presentation}

Keywords : Fetal MRI, brain malformations

\section{Fetal MRI in diagnosis of brain and spine malformations}

$\underline{\text { Ivana Markovic }}^{1}$-, Vesna Stokanovic ${ }^{1}$, Zoran Radovanovic ${ }^{1}$, Petar Bosnjakovic $^{2}$

Clinical Center Nis Center for Radiology Nis-Serbia ${ }^{1}$ Ibn Sina Hospital Radiology department Kuwait-Kuwait ${ }^{2}$

Prenatal diagnosis of congenital brain malformation primarily relies upon prenatal sonography as method of choice for screening due to its low cost, wide availability and safety. However, it's not without limitations such as poor visualisation depending on fetal position and scull ossification, low tissue definition, and operator variability. Advances in technology resulting in rapide pulse sequences enabled fetal MRI to become a significant and complementary tool in prenatal diagnosis. After detection of a brain abnormality on routine sonography, fetal MRI is used to confirm or exclude it, also allowing more precise characterisation and detection of associated malformations not seen on sonography. In this paper we address fetal MR imaging of brain and spine malformations. Also, a serie of 167 fetal MRI examinations, performed at our institution during a period of 6 years, is reviewed. Pregnant women between 20th and 36th week of gestation underwent MRI because of suspected fetal pathology, high risc or history of severe brain malformation in previous pregnancy. In 54 cases $(32,3 \%)$ MRI was normal and in 113 cases $(67,7 \%)$ fetal pathology was detected. Most frequent anomalies found were hydrocephalus $(37,1 \%)$ in five cases associated with other anomalies, corpus callosum anomalies $(30,1 \%)$, Chiari II malformation $(14,2 \%)$, cephaloceles $(12,4 \%)$ and schizencephaly $(8 \%)$. We also found two cases of anencephaly an one sacrococcygeal teratoma. Fetal MRI provided detailed visualisation of fetal neurostructures and detection of sonographically occult abnormalities, and significantly influenced pregnancy management in $11 \%$ of cases.
5-P.12

Category: Pediatric

Presentation Type : Poster Presentation

Keywords :

Non-enhanced MRI and MRA for endovascular treatment planning in Vein of Galen Malformation

$\underline{\text { Dan Meila }}{ }_{-}{ }_{-}$Nikola Duerr ${ }^{1}$, Heinrich Lanfermann ${ }^{2}$, Friedhelm Brassel ${ }^{1}$

Sana Kliniken Duisburg Department of Radiology and Neuroradiology Duisburg-Germany ${ }^{1}$ Medical School Hannover Department of Diagnostic and Interventional Neuroradiology Hannover-Germany ${ }^{2}$

Introduction: Vein of Galen Malformation (VGM) is a severe pediatric neurovascular disease with high-flow arteriovenous shunts. Treatment of choice is endovascular embolization, which usually requires multiple treatment sessions. Until now no cross-sectional study deals with the preoperative evaluation. We want to present our long-term experience with non-enhanced MRI and MRA for the endovascular treatment planning in VGM.

Methods: Out of 43 patients with VGM treated between 2003 and 2016 we retrospectively reviewed 189 MRI studies. 108 studies were used for statistical assessment; all amongst others with standard T2, arterial TOF MRA and T2 weighted sequences without flow compensation in different planes. The presence of nidus and all arterial feeder (posterior choroidal arteries, anterior choroidal arteries, pericallosal arteries, basilar tip/PCA and leptomeningeal supply) was evaluated. On the venous side we assessed - if possible - the main fistula point on a four quadrant system. We used a scoring scale $(1=$ poor, $2=$ moderate, $3=\operatorname{good}, 4=$ excellent $)$ for identification of the arterial feeder groups and venous fistula points. Gold standard for comparison was DSA.

Results: The nidus was sufficiently identifiable in all sequences. Arterial feeder groups were best seen in arterial TOF MRA and thin transversal T2 sequence without flow compensation. The main venous fistula point was best seen in TOF MRA and in coronal and transversal thin T2 sequences without flow compensation.

Conclusion: Angioarchitecture of VGM for endovascular treatment planning is best seen in arterial TOF MRA and in thin transversal T2 sequences without flow compensation.

\section{5-P.13}

Category: Pediatric

Presentation Type : Poster Presentation

Keywords : Fetal MRI, FLAIR imaging, Brain development, brain malformations, subplate, lamination

Added value of FLAIR images in the evaluation of brain anomalies

Mariana Cardoso Diogo ${ }^{1}$, Gerlinde M. Gruber ${ }^{6}$, Peter Brugger ${ }^{6}$, Gregor Kasprian $^{2}$, Michael Weber ${ }^{6}$, Daniela Prayer ${ }^{2}$

Centro Hospitalar de Lisboa Central Neuroradiology Lisboa-Portugal ${ }^{1}$ Medical University of Vienna Neuroradiology Vienna-Austria ${ }^{2}$

MRI is an established tool for evaluating the fetal central nervous system, and assessment of brain lamination is an essential part of it. The subplate is identified consistently until gestational week 28 on T2WI. FLAIR imaging holds theoretical advantages in assessing both the subplate (abundant free water)and lesions. The added value of this sequence has not been established. The aim of this study is to compare T2W FSE/FLAIR images in 
fetuses with brain malformations and assess the additional information provided by the FLAIR sequence.

Methods: Retrospective evaluation of 268 fetal MRI with brain anomalies. Only exams with availability of T2w FSE and FLAIR images in at least one equivalent plane were included. Each sequence was analyzed for identification of the subplate by lobes. Findings were compared in two age groups: gestational age (GA) $\leq 28$ and $>28$. It was further assessed if malformations were identifiable on $\mathrm{T} 2$ and/or FLAIR, and determined whether FLAIR images provided additional information.

Results: Mean GA was 28.2 weeks. In the higher GA group there was a significant difference $(\mathrm{p}<0.001)$ in subplate identification in all lobes in favor of FLAIR sequence. In the younger GA group, differences were significant in the frontal $(\mathrm{p}=0,006)$, parietal and occipital lobes $(\mathrm{p}<0.001)$. Most lesions were at least partially identifiable on FLAIR images (91\%), although better depicted on T2 in $29.2 \%$.FLAIR provided additional information in excluding lesions in $71.5 \%$.

Conclusion: FLAIR images provide additional information, particularly in assessment of lamination, with higher sensitivity to subplate visualization especially after GW 29.

\section{5-P.14 \\ Category: Pediatric \\ Presentation Type : Poster Presentation}

Keywords : Pediatric population, pituitary insufficiency, MRI, pons, posterior fossa

\section{A novel index indicating pituitary insufficiency: Pons ratio}

$\frac{\text { Ozum Tuncyurek }^{1}}{\text { Yelda Ozsunar }^{1}}{ }^{-}$Mehmet Turgut ${ }^{2}$, Tolga Unuvar ${ }^{3}$, R Shane Tubbs $^{4}$,

Adnan Menderes University Radiology Aydin-Turkey ${ }^{1}$ Adnan Menderes University Neurosurgery Aydin-Turkey ${ }^{2}$ Adnan Menderes University Pediatric Endocrinology Aydin-Turkey ${ }^{3}$ Seattle Science Foundation Neurosurgery Seattle-United States ${ }^{4}$

Purpose: Pituitary insufficiency (PI) needs further research to optimize treatment. The purpose of the present study is to investigate the possible relationship between PI and the anatomical position of the pons as revealed by magnetic resonance imaging (MRI) in the pediatric age group. Methods: Brain MRI and clinical data of 48 children with suspected PI ( 22 females, 26 males; median age $11.6 \pm 2.2$ years) were examined retrospectively. To estimate the location of the pons, the ratio of pons height over the axis between the dorsum sellae and the fourth ventricular hill in the sagittal plane (A) to the total height of the pons (B) was calculated (A/B, pons ratio). The pons ratios of children with or without a diagnosis of PI were then compared.
Results: Fifteen patients were diagnosed as PI and treated with growth hormone $(\mathrm{GH})$, while the remaining 33 were not so diagnosed. The mean pons ratio of the patients diagnosed as PI and given GH $(0.31 \pm 0.07$, range: 0.18 to 0.42 ) differed significantly from the mean pons ratio of those without PI $(0.26 \pm 0.06$, range: 0.17 to $0.44 ; p=0.018)$.

Conclusions: On the basis of our findings we suggest that this association is due to a constitutionally formless posterior fossa that is not substantial enough to place the entire rhombencephalon, despite a higher rate of midline deficiencies in children with PI. Keeping this in mind, we hope that radiological examinations of suspected PI cases will in future include spatial measurements of the posterior fossa for definitive diagnosis.

\section{5-P.15}

Category: Pediatric

Presentation Type : Poster Presentation

Predictive value of apparent diffusion coefficient (ADC) in hypoxic newborns treated with hypothermia

Maja Cimpersek $^{1}$, Nuska Pecaric Meglic ${ }^{1}$, Darja Paro Panjan ${ }^{2}$, Andreja Skofljanec $^{2}$, Katarina Surlan Popovic ${ }^{1}$

University medical center Clinical institute of radiology LjubljanaSlovenia ${ }^{1}$ University medical center Pediatric clinic Ljubljana-Slovenia ${ }^{2}$ Background: Hypothermia reduces hypoxic-ischemic brain injury seen on magnetic resonance (MR) imaging. Diffusion weighted imaging (DWI) with apparent diffusion coeficient (ADC) has an important role in predicting clinical outcome for infants treated with hypothermia.

Aim: To assess which region of $\mathrm{ADC}$ value measurement serves best for predicting clinical outcome at 1 month of age and to set the treshold ADC values.

Methods: ADC values were measured in the posterior limb of the internal capsule and thalamus (PLIC/T), basal ganglia (BG), white matter (WM), insular cortex (IC) and parietal cortex (PC). Clinical outcome was based on the Amiel-Tison neurologic assessment at 1 month of age. We used the Mann-Whitney $U$ test for every measured region. We also used ROC curve analysis to check at which ADC value, sensitivity and specificity for predicting clinical outcome are the best.

Result: Statistically significant difference between the group with good and bad clinical outcome was confirmed in PLIC/T and WM. Treshold $\mathrm{ADC}$ value, measured in PLIC/T is $920 \times 10-6 \mathrm{~mm} 2 / \mathrm{s}$ and in WM is $1153 \times 10-6 \mathrm{~mm} 2 / \mathrm{s}$.

Conclusions: We confirmed that ADC values measured in PLIC/T and WM are statistically significant. We also set the treshold $\mathrm{ADC}$ value for $\mathrm{PLIC} / \mathrm{T}$ and WM for predicting clinical outcome.ADC values in our study were higher than values from studies without hypothermia. Based on these results we conclude that hypothermia had a positive effect on HIE. 
AUTHOR INDEX

Abe, Toshi

Abelairas, Jose

Åberg, Laura

Ada, Emel

Adam, Gilles

Afat, Saif

Aguilar-Pérez, Marta

Aguilera, Carles

Alber, Georgina

Alberich, Manel

Alberts, Esther

Alcaine, Malaika Cordeiro

Ali, Ahmed

Alkadhi, Hatem

Alvarez-Linera, Juan

Alvarez-Muelas, Alberto

Ambady, Prakash

Amelin, Mikhail

Amelung, Nadine

Aoki, Takachika

Apaydin, Melda

Appuhamy, Chinthaka

Argyropoulou, Maria

Arslan, Ilker Burak

Arús, Carles

Askaner, Krister

Astrakas, Loukas

Auger, Cristina

Austein, Friederike

Autti, Taina

Avdagic, Edin

Avignone, Sabrina

Avsenik, Jernej

Aymerich, Aymerich

Badilla, Lautaro

Bae, Sohi

Ballenilla, Federico

Baltes, Jessica Katharina

Baltsavias, Gerasimos

Bañeras, Jordi

Bansal, Ashank

Bansemir, Kai

Baran, Agah

Bargallo, Nuria

Barronnet, Flore

Barsi, Péter

Barsic, Antonija Ruzic

Bartolini, Bruno

Bartolini, Chiras

Bäzner, Hansjörg

Behme, Daniel

Belkacem, Samia

Beltrán, Briggith

Bendszus, Martin

Bengzon, Johan

Bernal, Alexandra

Bette, Stefanie

Biarnes, Carles

Bier, Georg

Bink, Andrea

Biraschi, Francesco

Bisdas, Sotirios

Bladowska, Joanna
1-P.26, 2-P.09, 2-P.11

3-P.01

1-O.19

1-P.29, 1-P.42

1-P.63

1-P.56, 1-P.57

2-O.5

1-P.30

$1-\mathrm{O} .26$

1-O.11

$1-\mathrm{O} .26$

5-P.05

2-O.15

1-P.59

3-P.01

5-O.3, 3-P.01

1-P.24

1-P.38

1-P.61

1-P.26

1-P.52

2-P.16, 2-P.17

$1-0.15$

5-O.4

1-P.30

1-P.55

$1-0.15$

$1-0.11$

1-O.1

$1-0.19$

3-O.1, 4-O.6

$5-\mathrm{O} .1$

1-O.10

$1-\mathrm{O} .11$

1-P.65

1-O.4

1-P.21, 1-P.22, 1-P.23, 1-P.39

1-P.34

1-P.59

$1-\mathrm{O} .20$

2-P.17

2-O.5

$1-\mathrm{P} .42$

$1-\mathrm{O} .3$

2-P.03

$1-0.29$

1-O.24, 1-P.37

2-P.03

2-O.18

2-O.5

1-P.61

1-P.05

1-O.12, 1-P.67

2-O.13

1-O.6, 1-O.17

5-P.09

$1-\mathrm{O} .26$

1-O.12, 1-P.67

1-P.56

1-P.12

2-O.11

1-O.28, 1-P.10

$1-\mathrm{O} .25$
Blasco, Gerard

Boban, Jasmina

Boban, Nikola

Bocchio, Annapaola

Boeckh-Behrens, Tobias

Boito, Faustina Lalatta

Bonaccorsi, Raphaël

Bonneville, Fabrice

Borkowska, Julita

Borota, Ljubisa

Borrelli, Pasquale

Boskos, Christos

Bosnjakovic, Petar

Boto, José

Boudabbous, Sana

Bourcier, Romain

Boursianis, Themistoklis

Bozkurt, Öznur

Brakus, Alma

Brandstack, Nina

Brassel, Friedhelm

Bravo, Eduardo

Brkic-Georgievski, Biljana

Brockmann, Carolin

Brockmann, Marc A.

Brown, Martin

Brugger, Peter

Brunetti, Arturo

Buitrago Sánchez, Nelson Mauricio

Bulja, Deniz

Buono, Giuseppe

Burkhardt, Jan-Karl

Busaidi, Ayisha Al

Büsen, Martin

Cakir, Volkan

Camins, Àngels

Campeau, Norbert

Cannerfelt, Björn

Caron, Philippe

Castaño, Ana Maria

Castaño, Nohora

Castellan, Lucio

Castelletti, Lara

Castro, Mario

Caulo, Massimo

Cavusoglu, Berrin

Cazzola, Vanessa

Cebeci, Hakan

Cellerini, Martino

Cervo, Amedeo

Cha, Snag-Hoon

Chai, Jyh-Wen

Chang, De-Hua

Chang, Ni-Jung

Chapot, René

Charyasz, Edyta

Chatterjee, Fiona

Chelstowska, Sylwia

Chen, Hsian-Min

Chernov, Sergey

Chi-Chang Chen, Clayton

Chiras, Jacques
1-O.12, 1-P.67

5-P.10

5-P.10

1-P.19, 1-P.33

$1-0.26$

5-O.1

2-O.2

1-O.9, 1-P.63

5-P.09

2-P.05

1-O.21

1-P.10

5-P.11

1-O.27, 4-O.4

4-O. 4

2-O.4, 2-P.01

1-P.64

1-P.62

1-P.50

$1-0.19$

2-O.12, 5-O.6,

1-P.34, 2-P.07, 2-P.08, 5-P.12

4-O.5

1-P.32

2-O.16, 1-P.56, 1-P.57

1-P.56, 1-P.57

1-P.13

5-P.13

$1-\mathrm{O} .21$

5-P.05

3-O.1, 4-O.6

2-P.14

1-P.59

$1-\mathrm{O} .28$

2-O.16

1-P.52

1-P.30

1-P.17

$1-\mathrm{O} .5$

1-O.9

1-P.20

5-P.09

4-P.02

4-P.02

1-P.65

4-O.2, 1-P.48

1-P.29

1-P.63

5-P.08

2-O.11

1-O.21

1-P.16

1-P.43

3-P.02

1-P.43

2-O.9

1-O.28

1-O.18

5-Р.03, 5-Р.04

1-P.43

1-P.38

2-O.6, 1-P.43

2-O.2, 2-P.03, 2-P.06 
Choi Choi, Woo Suk

Choi, Chi-Hoon

Choi, See Sung

Chuang, Tzu-Chao

Chung, Wen-Yuh

Chyul Hong, Seung

Cimpersek, Maja

Cinnante, Claudia Maria

Claire, Toquet

Clarençon, Frédéric

Cocozza, Sirio

Cognard, Christophe

Comi, Giancarlo

Contreras, Cristina Utrilla

Cordera, Susanna

Cormier, Evelyne

Cowley, Peter

Crispino, Mario

Crnovrsanin, Faris

Cukurova, Ibrahim

Čulo, Branimir

Dablan, Ali

D'agostino, Vincenzo

Dajic, Dragan

Dakovic, Marko

Damjanovic, Dusan

Danyeli, Ayca Ersen

Daumas-Duport, Benjamin

Daunis-i-Estadella, Pepus

Davic, Kristina

Davidovic, Kristina

Dazinger, Florian

De Filippis, Federica

De La Vega, Felipe

Debska, Joanna

Degos, Vincent

Delattre, Bénédicte

Delehaye, Luigi

Delgado, Pilar

Dembowska-Baginska, Bozenna

Demir, Ozden

Desal, Hubert

Di Clemente, Loris

Di Egidio, Vincenzo

Di Maria, Federico

Didier, Dormont

Diogo, Mariana Cardoso

Djurdjevic, Tanja

Dogan, Orhan

Dormont, Didier

Drazyk, Anna

Duerr, Nikola

Duman, Özgür

Durmo, Faris

Duzgun, Fatih

Eaves, Scott

Echeverria, Daniel

Eguchi, Seiichiro

Ejma, Maria

Enblad, Per

Engelholm, Silke

Esposito, Gianluigi

Fabó, Dániel

Fabre, Nelly

Falini, Andrea

Faragò, Giuseppe
1-P.68

1-P.16

1-P.28

1-P.66

3-O. 2

2-O.3

5-P.15

5-O.1

2-O.17, 1-P.49

2-O.2, 2-O.18, 2-P.03, 2-P.06

$1-\mathrm{O} .21$

1-P.63

$1-\mathrm{O} .7$

5-P.05

1-P.33

2-O.2, 2-P.06

$1-\mathrm{O} .18$

2-O.11

1-P.25, 1-P.35

5-O. 4

2-P.02

1-O.8, 1-P.62

2-P.14

$1-\mathrm{O} .16$

1-P.06

1-P.06

1-P.42

2-O.4, 2-P.01

1-O.12, 1-P.67

1-P.35

1-P.06, 1-P.25

1-P. 27

2-O.14, 1-P.46

4-O.5

5-P.09

2-O.2

4-O. 4

2-P.14

$1-\mathrm{O} .20$

5-P.04

1-P.52

2-O.4,2-P.01

4-O.2, 1-P.48

2-O.14, 1-P.45, 1-P.46, 2-P.10

2-O.18, 2-P.03

1-P.05

5-P.13

1-P.27

$5-\mathrm{O} .4$

1-P.51

1-P.41

5-P.12

1-P.62

1-P.55

4-O.1

2-P.13

1-P.65

2-O.10

$1-\mathrm{O} .25$

2-P.05

1-O.6, 1-O.17, 1-P.55

1-P.45, 1-P.46, 2-P.10

$1-0.29$

1-P.63

$1-0.7$

2-O.11
Fernandez-Zubillaga, Amelia

Ferrier, Marine

Fezoulidis, Ioannis

Filippi, Massimo

Fisch, Loraine

Fleischer-Stepniewska, Katarzyna

Flore, Baronnet

Fogliani, Roberto

Fountas, Kostas

Förster, Alex

Frigerio, Michele

Fu, Jui-Hsun

Fukumoto, Hironori

Furfaro, Dany

Gabrieli, Joseph

Galanaud, Damien

Garcia-Dorado, David

Garrido, Diego

Garzón, Gonzalo

Gasparotti, Roberto

Gaudino, Chiara

Geerts, Bert

Gelal, Fazil

Gempt, Jens

Georgievski-Brkic, Biljana

Gich, Jordi

Gil, Miguel

Giorgianni, Andrea

Gizewski, Elke Ruth

Gkinis, Georgios

Glodny, Bernhard

5-O.3, 3-P.01

1-P.63

1-P.47

$1-\mathrm{O} .7$

1-P.13

$1-\mathrm{O} .25$

1-P.05

5-O.1

1-P.47

$1-\mathrm{O} .13$

2-O.11

1-P.66

2-O.10

1-P.33

2-O.18, 2-P.03

1-P.51

$1-\mathrm{O} .20$

5-O.3

5-O.3, 3-P.01

2-O. 11

2-O.13

1-P.51

1-P.52

1-O.26

1-P.36, 1-P.50, 1-P.54

1-O.12, 1-P.67

1-P.30

2-O.11

1-P.27

1-O.27

1-P.27

$1-\mathrm{O} .7$

$1-0.2$

1-P.63

1-P.27

Grams, Astrid Ellen

Greling, Björn

Grieb, Dominik

Groden, Christoph

Gruber, Gerlinde

Grunwald, Iris

Guidotti, Roberto

Guler, Ibrahim

Gunel, Mintaze Kerem

Guo, Wan-Yuo

Guyomarch, Béatrice

Guyomarch-Delasalle, Béatrice

Güleryüz, Handan

Gyebnár, Gyula

Ha Kim, Keon

Hakkarainen, Antti

Hamano, Eika

Hamilton, Bronwyn

Has, Arzu Ceylan

Haydee Corzo, Cecilia

Heine, Oliver

Henkes, Hans

Herazo, Cherina

Heringer, Sarah

Hernando, Jorge

Herweh, Christian

Hilario, Amaya

Hiltunen, Jaana

Hinzpeter, Ricarda

Hirohata, Masaru

Houchard, Aude

Hsu, Shuo-Hsiu
2-O.12, 5-O.6, 1-P.34, 2-P.08

$1-\mathrm{O} .13$

M. 5-P.13

2-P.13

1-P.48

5-P.08

$5-\mathrm{O} .2$

3-O.2

2-O.17, 1-P.49

2-O. 4

5-O.4

$1-\mathrm{O} .29$

2-O.3

$1-0.19$

2-O.7, 2-O.10

1-P.24

$5-\mathrm{O} .2$

2-O.16

$1-\mathrm{O} .13$

2-O.5

5-P.09

2-O.16

1-P.39

2-O.13

1-P.20, 1-P.21, 1-P.22, 1-P.23, 1-P.39

$1-0.19$

1-P.59

2-P.09, 2-P.11

$1-0.9$

1-P.66
2-O.12, 5-O.6, 1-P.34, 2-P.07, 2-P.08 
Huber, Thomas

Hubert, Desal

Huhndorf, Monika

Hui, Francis

Ihn, Yon-Kwon

Il Jo, Kyung

Inaoka, Tsutomu

Ince, Aydin

Inglot, Malgorzata 1-O.25

Ito, Yoshiro

Jager, Rolf

Jakab, Andras

Jansen, Olav

Jara, Marcelo

Javadpour, M.

Jean Michel, Serfaty

Jefic, Svjetlana

Jeon, Pyoung

Jeon, Se Jeong

Jevsek, Marko

Josep, Puig

Jovanovic, Marija

Jovanovski, Aleksandar

Jović, Andrijana

Jönsen, Andreas

Julià-Sapé, Margarida

Jung, Min Kyung

Jung, Won-Sang

Jurkiewicz, Elzbieta

K. Narayan, Anand

Kacar, Katarina

Kaesmacher, Johannes

Kalaitzakis, Georgios

Kalousek, Vladimir

Kamsu, Manuela

Kang, Hyunkoo

Kapsalaki, Eftychia

Karaali, Kamil

Karabay, Nuri

Karli Oguz, Kader

Karlović-Vidaković, Marijana

Kasakura, Shigen

Kasprian, Gregor

Kasuya, Shusuke

Kataoka, Hiroharu

Katase, Shichiro

Katayama, Reiji

Katsaros, Vasileios K.

Kavroulakis, Eleftherios

Kellenberger, Christian

Kızılay, Ferah

Killeen, Ronan

Kim, Dami

Kim, Eui Jong

Kim, Hee Yeong

Kim, Hye Jeong

Kim, Hyug-gi

Kim, Jinna

Kim, Tae Yoon

Kinhult, Sara

Kirschke, Jan

Kitamura, Noriko

Kitsos, George

Kleine, Justus

Klimaj, Zoltán

Klose, Uwe
$1-\mathrm{O} .26$

2-O.17, 1-P.49

1-O.1

2-P.16

1-P.03

2-O.3

$1-\mathrm{O} .14$

1-P.62

2-O.10

1-P.13

5-O.5

$1-\mathrm{O} .1$

1-P.65

1-P.41

2-O.17

4-O.3, 4-P.01

2-O.3

1-P. 28

2-P.04

$1-\mathrm{O} .12$

1-P.06

1-P.54, 2-P.15

2-P.02

1-O.5, 1-P.14

1-P.30

1-P.03

1-P.03

5-P.03, 5-Р.04, 5-P.09

$1-\mathrm{O} .8$

1-P.15, 1-P.31, 1-P.32

2-O.8

1-P.64

2-P.02

1-P.63

1-P.04, 1-P.08

1-P.47

1-O.8,1-P.60, 1-P.62

1-P.42

5-O.2

5-P.07

2-O.1

5-P.13

$1-\mathrm{O} .14$

2-O.10

1-O.2

1-P.26

1-P.10

1-P.64

5-O.5

$1-0.8$

1-P.41

1-P.09

1-P.68

5-P.02

5-P.02

1-P.68

$1-\mathrm{O} .4$

1-P.02

1-O.6, 1-O.17, 1-P.55

$1-0.26$

$1-\mathrm{O} .14$

$1-0.15$

2-O.8

$1-0.29$

$1-0.28$
Knauth, Michael

nezevic-Pogancev, Marija 5-P.10

Knoflach, Michael 1-P.27

Knysz, Brygida $1-0.25$

Kolić, Zlatko 5-P.07

Korchi, Amine 4-O.4

Kore, Laura $\quad$ 1-P.20

Koren, Laura 1-P.21, 1-P.22, 1-P.23, 1-P.39

Korkem, Duygu

Kosanovic, Tijana

Kostic, Dejan

Kostic, Irena

Kostic, Jelena

Kostic, Smiljana

Kotulska-Jozwiak, Katarzyna

Kovac, Natasa

Kovacevic, Milica

Kovacic, Slavica

Kozák, Lajos Rudolf

Kozcu, Sureyya Hikmet

Kozic, Dusko

Kumlien, Eva

Kuo, Kuei-Hong

Kurre, Wiebke

Kyrgias, George

La Tessa, Giuseppe

Lafè, Elvis

Lagares, Alfonso

Lai, Ping-Hong

Lai, Yen-Jun

L'allinec, Vincent

Lally, Frank

Lamió, Lluís

Lamot, Urka

Lampinen, Björn

Lancelot, Eric

Lanfermann, Heinrich

Lapucci, Caterina

Lätt, Jimmy

Lattanzi, Ruggero

Law-ye, Bruno

Lazeyras, François

Leclercq, Delphine

Lee, Inho

Lee, Kyung Mi

Lee, Seung-Koo

Lee, Wickly

Lenoble, Cédric

Lili, Détraz

Lilja, Åsa

Lin, Ching-Po

Lin, Huey-Shyan

Liouta, Evangelia

Lombardi, Sophie

Longo, Alja

Longoni, Marco

López, Ingeborg

Lövblad, Karl-Olof

Luo, Chao-Bao

Machado, Davide

Maciejewska-Kotz, Magdalena

Mahmoud, Ehab

Mahtout, Jugurtha

Majós, Carles

Maksimovic, Ruzica

Malczyk, Katarzyna
1-P.21, 1-P.22, 1-P.23, 1-P.39
5-O.2

1-P.54, 2-P.15

1-P.54, 2-P.15

1-P.25

1-P.25, 1-P.35

1-P.54

5-P.03, 5-P.09

5-P.10

1-P.11

1-O.24, 1-P.37

$1-0.29$

5-O.4

1-P.50, 5-P.10

$1-\mathrm{O} .16$

$1-0.22,3-0.2$

2-O.5

1-P.47

2-P.14

2-O.11

1-P.20

1-P.66

1-O.22

2-P.01

2-P.13

1-P.67

$1-\mathrm{O} .10$

1-O.6, 1-O.17

1-P.44

5-O.6, 5-P.12

4-P.02

1-O.5, 1-O.17, 1-P.55

2-O.14, 1-P.45, 1-P.46, 2-P.10

1-P.05

$1-\mathrm{O} .27$

1-P.05

1-P.09

1-P.68

1-O.4

2-P.16, 2-P.17

2-O.4, 2-P.01

2-O.17, 1-P.49

1-O.5, 1-O.6, 1-O.17, 1-P.14

1-P.43

1-P.66

1-P.10

5-O.1

1-P.18

2-O.11

1-P. 65

1-O.27, 4-O.4

2-O.6

1-P.19

5-P.09

2-P.05

2-O.2

1-P.30

1-P.06, 1-P.25

5-P.04 
Nowak, Katarzyna

Nyberg, Christoffer

Nystedt, Jessika

Odashima, Masayuki

Oguni, Eri

Ohara, Arisa

O'Hare, Alan

Oishi, Hidenori

Okuma, Cecilia

Olivo, Gaia

Orejas, Arancha Royo

Orita, Yoji

Ortiz, Nadia

Ostojic, Jelena

Othman, Ahmed E.

Ovali, Gulgun Yilmaz

Ozbiner, Huseyin

Ozsunar, Yelda

Öztek, Alp

Pabuscu, Yuksel

Paksoy, Yahya

Pakula-Kosciesza, Iwona

Palma, Giuseppe

Pan, Pan

Panara, Valentina

Panjan, Darja Paro

Papadaki, Efrosini

Papagiannaki, Chrysanthi

Papanikolaou, Nickolas

Pappa, Chrysavgi

Pareto, Deborah

Park, Dong Woo

Park, Yae-Won

Patsoura, Sofia

Pavia, Marco

Pawliszak, Piotr

Pedraza, Salvador

Pekcevik, Yeliz

Pellegrino, Carlo

Perek-Polnik, Marta

Peric, Predrag

Pero, Guglielmo

Peroni, Roberto

Persico, Nicola

Pesovic-Grbovic, Marija

Petrović, Slađana

Pham, Mirko

Piano, Mariangela

Piccirilli, Eleonora

Pisani, Antonio

Pisarevic-Neskovic, Jovanka

Piscitelli, Valeria

Pistocchi, Silvia

Pons, Albert

Popovic, Katarina Surlan

Popović, Mara

Pravata, Emanuele

Prayer, Daniela

Premat, Kévin

Puglielli, Edoardo

Puig, Josep

Pyatigorskaya, Nadya

Quilici, Luca

Radovanovic, Zoran

Radulac, Mišel

Raininko, Raili
5-P.09, 5-P.03, 5-P.04 2-P.05

1-O.5, 1-P.14

$1-\mathrm{O} .14$

5-P.01

1-O.2

1-P.41

2-O.7

1-P.65

$1-\mathrm{O} .21$

5-P.05

2-O.7, 2-O.10

$1-0.27$

1-P.50

1-P.56, 1-P.57

4-O.1

5-P.08

5-P.14

$1-\mathrm{O} .8$

4-O.1

5-P.08

5-P.03

$1-\mathrm{O} .21$

$1-\mathrm{O} .22$

1-P.48, 4-O.2

5-P.15

1-P.64

2-O. 4

1-P.10

$1-\mathrm{O} .15$

1-O.11, 1-O.20

1-P.02

$1-\mathrm{O} .4$

1-P.63

2-O.11

5-P.03, 5-P.04

1-O.12, 1-P.67

5-O. 4

2-O.11

5-P.04

1-P.54

2-O.11

2-O.11

$5-\mathrm{O} .1$

$1-\mathrm{O} .23$

3-O.4, 1-P.40

2-O.13

2-O.11

4-O.2

$1-\mathrm{O} .21$

1-P.31

2-P.14

2-O.18, 2-P.03

1-P.30

5-P.15

5-P.06

$1-\mathrm{O} .7$

5-P.13

2-O. 2, 2-P.03

2-O.14, 1-P.45, 1-P.46, 2-P.10

$1-P .67$

1-P.05, 1-P.51

2-O.11

5-P.11

2-P.15

$1-\mathrm{O} .16$
Mannfolk, Peter

Mansour, Ossama

Mardighian, Dikran

Maréchal, Bénédicte

Marić, Daliborka

Marileo, Roberto

Maris, Thomas

Marković, Ivana

Mårtensson, Johan

Martin, Patricia

Martinez-Moreno, Rosa

Maruyama, Daisuke

Massimo, Rosset

Masulovic, Dragam

Matsubara, Hirofumi

Mattei, Peter Angelo

Mauricio Buitrago, Nelson

McGuigan, Chris

Medenica, Svetlana Milosevic

Meglic, Nuska Pecaric

Mehrabi, Sara

Meila, Dan

Melber, Katharina

Meloni, Teodoro

Michel, Serfaty Jean

Migliaccio, Silvia

Milatovic, Biljana

Milenkovic, Gordana

Miletic, Damir

Millan, Jose Maria

Miller, Gary

Milosevic, Aleksandra

Milosevic-Medenica, Svetlana

Misovic, Miroslav

Miyamoto, Susumu

Miyazaki, Isao

Mizuguchi, Masashi

Mlakar, Jernej

Moles, Alexis

Moll, Gonzalo Garzón

Montalban, Xavier

Montoya, Francisca

Mora, Juan Andres

Mori, Takahisa

Morioka, Hotohiro

Möhlenbruch, Markus

Müller, Marguerite

Nadir, Mustafa

Nagata, Manabu

Nagata, Shuji

Nakai, Noriyoshi

Nakatsuka, Tomoya

Nakayama, Yasuhide

Navarra, Riccardo

Neidlin, Michael

Nemoto, Shigeru

Neuwelt, Edward

Nieto, Karen Natalia

Nikolaou, Konstantin

Nikolic, Igor

Nikolic, Ivan

Nikos Psychogios, Marios

Nikoubashman, Omid

Nilsson, Petra

Niry, Dana

Nordmeyer, Hannes
1-P.14

2-O.15, 1-P.53

2-O.11

$1-0.27$

4-O.3, 4-P.01

1-P.65

1-P.64

1-P.40, 5-P.11

$1-0.5$

1-P.20, 1-P.21, 1-P.22, 1-P.23, 1-P.39

2-O.5

2-O.10

1-P.19

1-P.35

2-O.10

4-O.2, 1-P.48

3-P.01

1-P.41

1-P.07

5-P.15

5-P.06

2-O.12, 5-O.6, 1-P.34, 2-P.07, 2-P.08, 5-P.12

2-O.12, 5-O.6, 1-P.34, 2-P.07, 2-P.08

1-P.33

1-P.49

$1-\mathrm{O} .21$

1-P.07

1-O.23, 1-P.36

1-O.24, 1-P.37

1-P.22

1-P.17

1-P.15

1-P.11

1-P.54

3-O.3

$1-\mathrm{O} .2$

5-P.01

5-P.06

2-P.01

5-P.05

$1-\mathrm{O} .11$

4-O.5, 1-P.65

5-P.09

2-O.1

2-P.09, 2-P.11

$2-0.13$

2-O.16, 1-P.56, 1-P.57

1-P.42

3-O.3

1-P.26

2-O.1

$1-0.14$

2-O.7

1-P.48

2-O.16

2-P.12

1-P.24

5-P.09

1-P.57

1-P.07

1-P.06, 1-P.35

1-P.61

2-O.16, 1-P.56, 1-P.57

1-O.5, 1-P.14

1-P.13

2-O.9 
Ramió, Lluís

Ramos, Ana

Redon, Richard

Reganati, Paolo

Rehwald, Rafael

Remida, Paolo

Riccio, Eleonora

Riccitelli, Gianna

Riedel, Christian

Ringel, Florian

Ristic, Aleksandar

Rivero, Mireia

Riviere, Louis-David

Rizzuti, Tommaso

Rocca, Mara

Roche, Alexis

Roder, Constantin

Roffe, Christine

Roh, Keuntak

Romain, Bourcier

Ronne-Engström, Elisabeth

Rosano, Anna

Rosset, Massimo

Rosso, Charlotte

Rotim, Krešimir

Rovira, Alex

Royo, Arantxa

Royo, Aranzazu

Rudas, Gábor

Rumboldt, Zoran

Rydelius, Anna

Rymer, Weronika

Ryu, Hye Ji

Rzaev, Jamil

Sadowski, Krzysztof

Saher, Mohamed

Sahin, Hilal

Saito, Naoko

Saitta, Laura

Sakai, Nobuyuki

Sakamoto, Rokudai

Saleh, Mahdi

Salvador, Elena

Salvedra, Judith

Samson, Yves

Sánchez-Fernández, Juan J.

Sarıaltın, Zafer

Sasiadek, Marek

Sastre-Garriga, Jaume

Satow, Tetsu

Savaki, Helen

Savas, Derya Durusu Emek

Savić, Ostoja

Savic, Zivorad

Scheer, Ianina

Schertz, Mathieu

Schiestl, Thomas

Schlunz-Hendann, Martin

Schlunz-Hendann, Martin

Schmid, Elisabeth

Schmidt, Cynthia

Schubert, Gerrit Alexander

Scola, Elisa

Sehic, Adnan

Sekulić, Igor

Senol, Utku
$1-0.12$

1-P.20, 1-P.21, 1-P.22, 1-P.23, 1-P.39

2-O. 4

$2-\mathrm{O} .11$

1-P.27

2-O.11

$1-\mathrm{O} .21$

$1-0.7$

$1-\mathrm{O} .1$

$1-\mathrm{O} .26$

1-P.06

1-O.12, 1-P.67

$1-0.9$

5-O.1

$1-\mathrm{O} .7$

$1-0.27$

$1-\mathrm{O} .28$

2-P.13

1-P.08

2-O.17, 1-P.49

2-P.05

1-P.33

1-P.33

2-P.03

2-P.02

$1-\mathrm{O} .11,1-\mathrm{O} .20$

$5-\mathrm{O} .3$

3-P.01

$1-\mathrm{O} .29$

1-O.24, 1-P.37

1-O.6, 1-O.17, 1-P.55

$1-\mathrm{O} .25$

1-P.28

1-P.38

5-P.03

$1-\mathrm{P} .53$

5-O.4

1-P.01

4-P.02

2-O.7

2-P.09, 2-P.11

2-P.13

1-P.20, 1-P.21, 1-P.22, 1-P.23, 1-P.39

1-O.12, 1-P.67

2-P.03

1-P.30

1-P.60

$1-\mathrm{O} .25$

$1-\mathrm{O} .11$

2-O.7, 2-O.10

1-P.64

1-P.29

4-O.3

1-P.35

5-O.5

1-P.05

1-P.27

2-O.12

1-P.34, 2-P.07, 2-P.08

$2-0.5$

5-O.6

1-P.56

5-O.1

4-O.6

2-P.15

1-O.8, 1-P.60
Seong Choi, Yoon

Sert, Caroline

Seruga, Tomaz

Shy, Cherng-Gueih

Sicignano, Carmine

Simó, Rafael

Simos, Panagiotis

Sindel, Hakkı Timur

Sirabella, Giovanni

Skofljanec, Andreja

Smits, Marion

Sokic, Dragoslav

Song, Chang June

Soo Kim, Jong

Soo Kim, Min

Sourour, Nader

Stankovic, Ana

Stankovic, Danilo

Steinseifer, Ulrich

Stippich, Christoph

Stojanov, Dragan

Stojanovic, Sanja

Stojković, Nikola

Stokanovic, Vesna

Stosic-Opincal, Tatjana

Strandberg, Olof

Stranjalis, George

Strocchi, Sabina

Sundgren, Pia

Szidonya, Laszlo

Takagi, Takashi

Takahashi, Jun

Takahashi, Jun C

Takata, Megumu

Tanaka, Norimitsu

Tanaka, Shunichi

Tanno, Yuhei

Tarhan, Serdar

Tasic, Goran

Tatagiba, Marcos

Tedeschi, Enrico

Teodoro, Meloni

Terada, Hitoshi

Terzic-Beljakovic, Aleksandra

Thust, Steffi

Tintoré, Mar

Tokola, Anna

Toović, Gordana

Torres, Francisco

Tramontini Jens, Carolina

Trickovic, Jelena

Triulzi, Fabio

Trpcevic, Radivoje

Tsogkas, Ioannis

Tsuchiya, Kazuhiro

Tubbs, R Shane

Tul, Nataa

Tulupov, Andrey

Tuncyurek, Ozum

Turgut, Mehmet

Tuura-O, Ruth

Uchino, Akira

Uchiyama, Yusuke

Ulyte, Agne

Unuvar, Tolga

Urlan Popovič, Katarina
1-O.4

1-O.9

2-P.04

2-O.6

2-P.14

$1-\mathrm{O} .20$

1-P.64

1-P.62

2-P.14

5-P.15

$1-\mathrm{O} .3$

1-P.06

1-P.09

2-O.3

2-O.3

2-O.18, 2-P.03

1-P.25, 1-P.35

1-P.25, 1-P.35

2-O.16

1-P.12

1-P.40

1-P.50

1-P.40

5-P.11

1-P.36

1-P.14

1-P.10

2-O.11

1-O.5, 1-O.6, 1-O.17, 1-P.14, 1-P.55

1-P.24

3-O.3

2-O.7, 5-P.01

2-O.10

3-O.3

1-P.26

2-O.10

2-O.1

4-O.1

1-P.07, 1-P.15

$1-0.28$

1-O.21

1-P.19

1-O.14

1-O.23

1-O.3

1-O.11

1-O.19

1-P.40

1-P.65

5-P.09

1-P.32

5-O.1

1-P.31, 1-P.32

1-P.47, 1-P.61

$1-\mathrm{O} .2$

5-P.14

5-P.06

1-P.38

5-P.14

5-P.14

5-O.5

1-P.01

1-P.26

1-P.10

5-P.14

1-O.10 
Urueña, Piedad

Usinskiene, Jurgita

Utrilla, Cristina

Ünal, Ali

Valavanis, Antonios

Valentina, Mileusnic

Valsasina, Paola

Valvassori, Luca

van Westen, Danielle

Varallyay, Csanad

Vargas, Maria Isabel

Vernoij, Meike

Vesnaver, Tina Vipotnik

Vidal, Aaron

Viguier, Alain

Vitosevic, Filip

Voicu, Ioan Paul

Vrabec, Matej

Vukomancic, Biljana

Waliszewska-Prosol, Marta

Wang, Po-Chin

Weber, Michae

Weber, Ralph

Webering, Nadine

Werring, David

Wiesmann, Martin
5-P.09

1-P.10

5-O.3, 3-P.01

1-P.60

1-P.59

1-P.15

$1-0.7$

2-O.11

$1-\mathrm{O} .17$

1-P.24

1-O.27, 4-O.4

$1-\mathrm{O} .3$

1-P.18, 5-P.06

1-P.65

1-P.63

1-P.11

1-P.48

$1-\mathrm{O} .10$

$1-0.23$

$1-0.25$

1-P.66

$15-\mathrm{P} .13$

2-O.9

2-0.9

$1-0.18$

2-O.16, 1-P.56, 1-P.57
Wilson, Duncan

Winklhofer, Sebastian

Wolf, Michael

Wong, Ho-Fai

Woo Park, Dong

Woo, Ji Young

$\mathrm{Wu}$, Chih-Chun

Wuu, Hsui-Mei

Yamamoto, Haruko

Yang, Ik

Ye, Bruno Law

Yener, Gorsev

Yi, Kyung Sik

Yoo, Inkyu

Yoshida, Ayako

Yoshida, Kazumichi

Yoshida, Kensaku

Yoshioka, Kazuhiro

Young Yeon, Je

Yousry, Tarek

Žigić, Vedran

Zikou, Anastasia

Zimmer, Claus

Zimny, Anna

Zubillaga, Amelia Fernández

Zupanc, Tatjana Avič
1-O.18

1-P.59

$1-\mathrm{O} .13$

2-O.6

3-P.03

5-P.02

$3-\mathrm{O} .2$

3-O.2

2-O.7

5-P.02

1-P.51

1-P.29

1-P.16

1-P.09

1-P.26

3-O.3

1-P.58

2-O.1

2-0.3

1-O.3

1-P.50

$1-\mathrm{O} .15$

1-O.26

1-O.25

5-P.05

5-P.06 